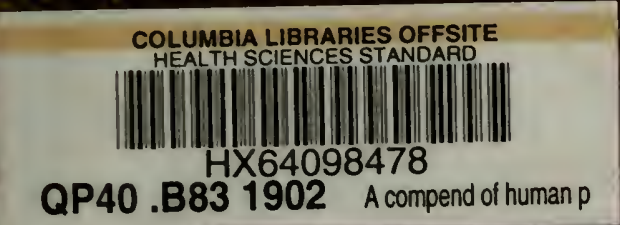

\title{
RECAP
}

$$
\begin{aligned}
& \text { OUIZ-COMPENDS } \\
& \text { PHysIOLOGY } \\
& \text { Dr. BRubaker. }
\end{aligned}
$$




\section{MORRIS' ANATOMY.}

SECOND EDITION.

791 Illustrations, of which 214 are Colored.

Human Anatomy. A Complete Systematic Treatise by Various Authors, Including Special Sections on Surgical and Topographical Anatomy, the Skin, and Vestigial and Abnormal Structures. Edited by Henry Morris, M.A. and

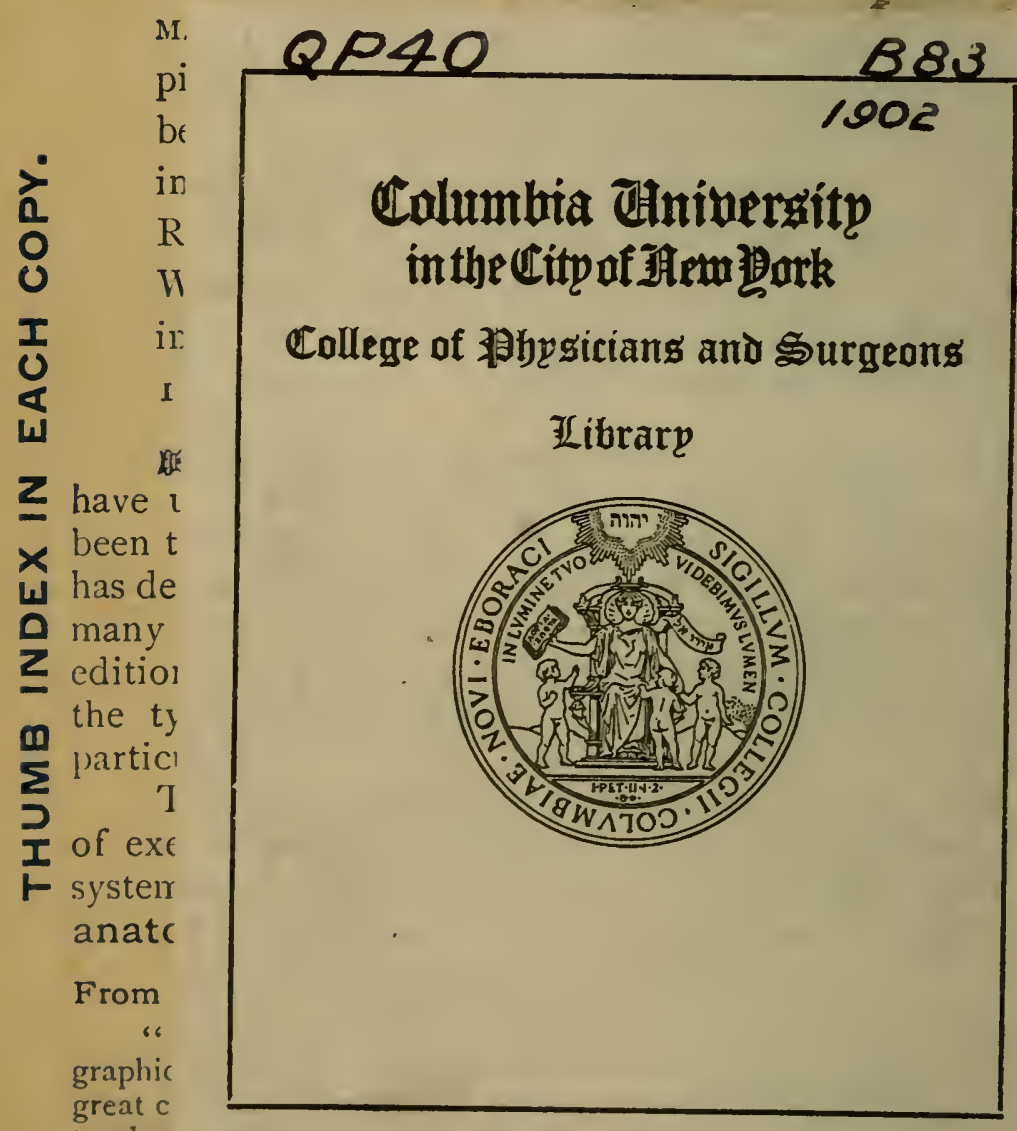

ex Hos-

; Mem-

Exam-

Edition.

ughout.

re Orig-

Octavo.

$t, \$ 7.00$

blishers text has e editor s whole; he first s, while several

cellence text is ook on

and topoented with book with be recom.

teachel

mended to all interested."

From The Philadelphia Medical Journal.

"Of all the text-books of moderate size on human anatomy in the English language, Morris' is undoubtedly the most up-to-date and accurate. . . . For the student, the surgeon, or for the general practitioner who desires to review his anatomy, Morris' is decidedly the book to buy."

A Descriptive Circular of Morris" "Anatomy," with Sample Pages and Colored Illustrations, will be sent free to any address. 


\section{Tyson's Practice of Mzdicine SECOND EDITION.}

\section{Illustrations, Several of which are if Colors.}

The Practice of Medicine. A Text-Book for Kiysicians and Students, with Special Reference to Diagnoss and Treatment. By Janes Tyson, M.D., Professor of Medicine in the University of Pennsylvania; Physician the University and to the Philadelphia Hospitals; Fllow of the College of Physicians of Philadelphia; Menber of the Association of American Physicians, etc. Second Fition. Octavo. 1222 pages. I 27 Illustrations. CEoth, net, $\$ 5.50$; Sheep, net, $\$ 6.50$

This, edition has been entirely reset from new type. The author has revised it carefully and thoroughly, and added much new material and 37 new illustrations.

\section{From The Therapeutic Gazette.}

"From the first to the last of this large volume of nearly 1200 pages we find much to commend, almost nothing to criticise, and certainly nothing to contradict.

"It is in the writing and preparation of a work of this character that Dr. Tyson stands pre-eminent. Those of the profession-and t $\mathrm{t}^{2} \mathrm{rc}$ are many at this time-who have been fortunate enough to have been his pupils during their medical student days, will remember that he brought to his lectures and to his writings an amount of industry and care which many other tenchers failed to bring; and those who know him best as an author and teacher have expected that his book on the Practice of Medicine, when it appeared, would be a credit to himself and would increase his reputation as a medical author. This belief has proved correct. . . . We look forward to using this volume upon the 'Practice of Medicine' more than any of the others which grace our library shelves, and they are many and all of them good."

\section{From The North American Practitioner, Chicago.}

"The individuality of the writer is clearly manifest in the clear and practical manner in which diseases are described and their treatment expressed. . . The succeeding sections upon Diseases of the Digestive, Respiratory, Circulatory, and Nervous Systems, together with those of the Blood, Urinary Organs, Constitutional Diseases, etc., are so full as to well serve, in each instance, the needs of the specialist, while their grouping in one complete volume renders it a valuable text-book for students, and the one above any other now at command most suited to the present needs of the active practitioner. It is the best representative of the practical application of modern research and discovery to the treatment of diseases now at the command of the medical profession." 
Gould's

Medical

The Standard Medical

Reference Books.

Dictionaries.

130,000 HAVE

BEEN SOLD.

BY GEORGE M. GOULD, A.M., M.D.,

Editor of American Medicine.

THE ILLUSTRATED DICTIONARY OF MEDICINE, BIOLOGY, AND ALLIED SCIENCES. INCLUDING THE PRON.UNCIATION, ACCENTUATION, DERIVATION, AND DEFINITION OF THE TERMS USED IN MEDICINE AND THOSE SCIENCES COLLATERAL TO IT: BIOJ.OGY (ZOOLOGY AND BOTANY), CHEMISTRY, DENTISTRY, PHARMACOLGGY, MICROSCOPY, ETC. With many Useful Tables and numerous Fine Illustrations. Large Square Octavo. I633 pages. Fifth Edition now ready. Full Sheep, or Half Dark-Green Leather, net, $\$ 10.00$; With Thumb Index, net, \$I I.00; Half Russia, Thumb Index, net, \$1 2.00

THE STUDENT'S MEDICAL DICTIONARY. INCLUDING ALL THE WORDS AND PHRASES GENERALLY USED IN MEDICINE, WITH THEIR PROPER PRONUNCIATION AND DEFINITIONS, BASED ON RECENT MEDICAL LITERATURE. With Tables of the Bacilli, Micrococci, Leukomains, Ptomains, etc., of the Arteries, Muscles, Nerves, Ganglia, and Plexuses; Mineral Springs of the U. S., etc. Eleventh Edition, Illustrated. Revised, Enlarged by over 150 pages. Small Square Octavo. Half Lark Leather, net, \$2.50; with Thumb Index, net, $\$ 3.00$

THE POCKET PRONOUNCING MEDICAL LEXICON. 30,000 words pronounced and defined. Containing all the Words, their Definition and Pronunciation that the Student generally comes in contact with; also elaborate Tables of the Arteries, Muscles, Nerves, Bacilli, etc., etc.; a Dose List in both English and Metric Systems, etc., arranged in a most convenient form for reference and memorizing. Fourth Edition, 837 pages. $64 \mathrm{mo}$.

Full Limp Leather, Gilt Edges, net, \$1.00; Thumb Index, net, $\$ 1.25$

THE POCKET CYCLOPEDIA OF MEDICINE AND SURGERY. Edited by Drs. George M. Gould and W. L. Pyle. A Concise Practical Handbook containing a vast amount of Information Systematically Arranged so as to be of the Greatest Service to the Student. Uniform with "Gould's Pocket Dictionary." $64 \mathrm{mo}$.

Full Limp Leather, Round Corners, Gilt Edges, net, $\$ 1.00$

Thumb Index, net, \$1.25

Full discriptive circulars and sample pages sent free upon application. 
HUMAN PHYSIOLOGY

ELEVENTH EDITION

\author{
BRUBAKER
}


From The Southern Clinic.

"We know of no series of books issued by any house that so fully meets our approval as these? Quiz-Compends?. They are well arranged, full, and concise, and are really the best line of text-books that could be found for either student or practitioner."

\section{BLAKISTON'S ?QUIZ=COMPENDS?}

\section{The Best Series of Manuals for the Use of Students.}

Price of each, Cloth, $\$ 0.80$ net. Interleaved, for taking Notes, $\$ 1.00$ net.

These Compends are based on the most popular text-books and the lectures of prominent professors, and are kept constantly revised, so that they may thoroughly represent the present state of the subjects upon which they treat.

The authors have had large experience as Quiz-Masters and attaches of colleges, and are well acquainted with the wants of students.

nce They are arranged in the most approved form, thorough and concise, containing over 600 fine illustrations, inserted wherever they could be used to advantage.

Can be used by students of any college. shape.

They contain information nowhere else collected in such a condensed, practical

No. 1. POTTER'S ANATOMY. Sixth Revised and Enlarged Edition. Including Visceral Anatomy. Can be used with either Morris' or Gray's Anatomy. II 7 Illustrations and 6 Lithographic Plates of Nerves and Arteries, with Explanatory Tables, etc.

No. 2. HUGHES. PRACTICE OF MEDICINE. Part I. Sixth Edition, Revised, Enlarged, and Improved.

No. 3. HUGHES. PRACTICE OF MEDICINE. Part II. Sixth Edition, Revised, Enlarged, and Improved. These two books furnish a complete set of notes on the Practice of Medicine, including Nervous and Mental Diseases.

No. 4. BRUBAKER. PHYSIOLOGY. IIth Edition, with new Illustrations and a Table of Physiological Constants. Enlarged and Revised.

to. 5. LANDIS. OBSTETRICS. Sixth Edition. Revised and Edited by WM. H WELLS, M.D., Instructor Jefferson Medical College, Philadelphia. 47 Illustrations.

No. 6. POTTER. MATERIA MEDICA, THERAPEUTICS, AND PRESCRIP. TION WRITING. Sixth Revised Edition.

No. 7. WELLS. GYNACOLOGY. Second Edition. With many Illustrations.

No. 8. GOULD and PYLE. DISEASES OF THE EYE AND REFRACTION. Including Treatment and Operations and a Section on Local Therapeutics. With Formulze and rog Illustrations, several of which are in Colors. Second Edition.

No. 9. HORWITZ'S SURGERY, Minor Surgery, and Bandaging. Fifth Edition, Enlarged and Improved. With 98 Formula and $x 67$ Illustrations.

No. 10. LEFFMANN. CHEMISTRY. Inorganic and Organic. Fourth Edition. Including Urinalysis, Animal Chemistry, Chemistry of Milk, Blood, Tissues, the Secretions, etc.

No. 1x. STEWART. PHARMACY. Fifth Edition. Based upon Prof. Remington's Text-Book of Pharmacy.

No. 12. BALLOU. VETERINARY ANATOMY AND PHYSIOLOGY. With 29 graphic Illustrations.

No. 13. WARREN. DENTAL PATHOLOGY AND DENTAL MEDICINE. Third Edition, Illustrated. Containing all the most noteworthy points of interest to the Dental Student, and a Section on Emergencies.

No. 14. HATFIELD. DISEASES OF CHILDREN. Colored Plate. Third Edition, Revised and Enlarged.

No. 15. THAYER. GENERAL PATHOLOGY. With 78 Illustrations.

No. 16. SCHAMBERG. DISEASES OF THE SKIN. Second Edition. Illus.

No. 17. CUSHING. HISTOLOGY. With many Illustrations.

No. 18. THAYER. SPECIAL PATHOLOGY. With 34 Illustrations.

Price, each, $\$ 0.80$ net. Interleaved, for taking Notes, $\$ 1.00$ net.

P. BLAKISTON'S SON \& CO., PUBLISHERS,

PHILADELPHIA. 
?QUIZ-COMPENDS? NO. 4.

\section{A COMPEND}

OF

\section{HUMAN PHYSIOLOGY}

ESPECIALLY ADAPTED FOR THE USE OF MEDICAL STUDENTS

BY

ALBERT P. BRUBAKER, A.M., M.D.

ADJUNCT PROFESSOR OF PHYSIOLOGY AND HYGIENE IN THE JEFFERSON MEDICAL COLLEGE; PROFESSOR OF PHYSIOLOGY IN THE PENNSYLVANIA COLLEGE OF DENTAL SURGERY; LECTURER ON ANATOMY AND PHYSIOLOGY IN THE DREXEL * INSTITUTE OF ART, SCIENCE, AND INDUSTRY; FELLOW OF THE COLLEGE OF PHYSICIANS OF PHILADELPHIA.

ELEVENTH EDITION, REVISED AND ENLARGED

WITH ILLUSTRATIONS

AND

A TABLE OF PHYSIOLOGIC CONSTANTS

\section{PHILADELPHIA \\ P. BLAKISTON'S SON \& CO.}

IOI 2 WALNUT STREET

1902 
Entered according to Act of Congress, in the year 1902, by P. BLAKISTON'S SON \& CO.

In the Office of the Librarian of Congress, at Washington, D. C.

QP yO
383
1902 


\section{PREFACE TO THE ELEVENTH EDITION.}

A NEw edition of the Compend of Physiology having been called for the opportunity has again been taken to revise some of the old, and to insert some new paragraphs to various parts of the text, changes which it is believed will be of value to the student during his attendance on the lectures. It is hoped the Compend will continue to meet the needs of the medical student.

September I, $\mathbf{r}_{902 .}$

Albert P. Brubaker. 



\section{TABLE OF CONTENTS.}

PAGE,

INTRODUCTION, . . . . . . . . . . . . . . . . . . . . 9

General Structure of the Animal Body, . . . . . . . . . . I I

Chemic Composition of the Human Body, . . . . . . . . . . I5

Physiology of the Cell, . . . . . . . . . . . . . . . . 33

Histology of the Epithelial and Connective Tissues, . . . . 39

Mechanism of THE Skeleton, . . . . . . . . . . . . . 46

General Physiology of Muscular Tissue, . . . . . . . . 50

Special Physiology of Muscles, . . . . . . . . . . . . 63

Physiology of Nerve Tissue, . . . . . . . . . . . . . . 69

Foods AND Dietetics, . . . . . . . . . . . . . . . . 86

Digestion, . . • . . . . . . . . . . . • . . . 95

AbSORPTION, . . . . . . . . . . . . . . . . . . III

BloOD, . . . . . . . . . . . . . . . . II9

Circulation of the Blood, . . . . . . . . . . . . . . . I 25

Respiration, . . . . . . . . . . . . . . . . . I35

Animal Heat, . . . . . . . . . . . . . . . . . . . . I43

SECretion, . . . . . . . . . . . . . . . . . . . . I45

Mammary Glands, . . . . . . . . . . . . . . . I48

Vascular Glands, . . . . . . . . . . . . . . . I50

Excretion, . . . . . . . . . . . . . . . . . I55

Kidneys, . . . . . . . . . . . . . . .. . . I55

Liver, . . . . . . . . . . . . . . . . . I6 3

Skin, . . . . . . . . . . . . . . . I68

Cerebro-Spinal Axis, . . . . . . . . . . . . . . . . I7 I

SPINAL CORD, . . . . . . . . . . . . . . . . . 172

SPINAL NER VES, . . . . . . . . . . . . . . . I74 
Cranial Nertes, . . . . . . . . . . . . . . I 83

Medulla Oblongata, . . . . . . . . . . . . 197

Pons Varolit, ................... 20I

Crura Cerebri, . . . . . . . . . . . . 202

CorPora QUadrigemina, . . . . . . . . . . . . 203

Corpora Striata and Optic Thalami, . . . . . . . 204

Cerbbellum, . . . . . . . . . . . . . . 205

Cerebrum, . . . . . . . . . . . . . . . 207

Cerebral Localization of Function, . . . . . . . . 214

Sympathetic Nerious System, . . . . . . . . . 218

SENSE OF TOUCH, . . . . . . . . . . . . . . 222

Sense of TASTe, . . . . . . . . . . . . . . . . 223

SeNSE of SMell, . . . . . . . . . . . . . . 225

SENSE OF SIGHT, . . . . . . . . . . . 226

SENSE OF HeARING, . . . . . . . . . . . . . 237

Volce and Speech, . . . . . . . . . . . . . 245

EMBRYOLOGY, .................. . . . 248

Generative Organs of the Female, . . . . . . . . . 248

Generative Organs of the Male, .......... . 251

Development of Accessory Structures, . . . . . . . 252

Development of the Embryo, . . . . . . . . . 257

Table of Physiologic Constants, . . . . . . . . . 263

Table Showing Relation of Weights and Measures, . . . . 266

INDEX, ................... . . 267 


\section{A COMPEND}

oF

\section{HUMAN PHYSIOLOGY.}

Introduction.-An animal organism in the living condition exhibits a series of phenomena which relate to growth, movement, mentality, and reproduction. During the period preceding birth, as well as during the period included between birth and adult life, the individual grows in size and complexity from the introduction and assimilation of material from without. Throughout its life the animal exhibits a series of movements, in virtue of which it not only changes the relation of one part of its body to another, but also changes its position in space. If, in the execution of these movements, the parts are directed to the overcoming of opposing forces, such as gravity, friction, cohesion, elasticity, etc., the animal may be said to be doing work. The result of normal growth is the attainment of a physical development that will enable the animal, and, more especially, man, to perform the work necessitated by the nature of its environment and the character of its organization. In man, and probably in lower animals as well, mentality manifests itself as intellect, feeling, and volition. At a definite period in the life of the animal it reproduces itself, in consequence of which the species to which it belongs is perpetuated.

The study of the phenomena of growth, movement, mentality, and reproduction constitutes the science of Animal Physiology. But as these general activities are the resultant of and dependent on the special activities of the individual structures of which an animal body is composed, Physiology in its more restricted and generally accepted sense is the science which investigates the actions or functions of the individual organs and tissues of the body and the physical and chemic conditions which undẹrlie and determine them. 
This may naturally be divided into :

I. Special phy'siology, the object of which is a study of the vital phenomena or functions exhibited by the organs of any individual animal.

2. Comparative physiology', the object of which is a comparison of the vital phenomena or functions exhibited by the organs of two or more animals, with a view to unfolding their points of resemblance or dissimilarity.

Human physiology is that department of physiologic science which has for its object the study of the functions of the organs of the human body in a state of health.

Inasmuch as the study of function, or physiology, is associated with and dependent on a knowledge of structure, or anatomy, it is essential that the student should have a general acquaintance not only with the structure of man, but with that of typical forms of lower animal life as well.

If the body of any animal be dissected, it will be found to be composed of a number of well-defined structures, such as heart, lungs, stomach, brain, eye, etc., to which the term organ was originally applied, for the reason that they were supposed to be instruments capable of performing some important act or function in the general activities of the body. Though the term organ is usually employed to designate the larger and more familiar structures just mentioned, it is equally applicable to a large number of other structures which, though possibly less obvious, are equally important in maintaining the life of the individual-e. $g_{\text {. }}$, bones, muscles, nerves, skin, teeth, glands, blood-vessels, etc. Indeed, any complexly organized structure capable of performing some function may be described as an organ. A description of the various organs which make up the body of an animal, their external form, their internal arrangement, their relations to one another, constitutes the science of Animal Anatomy.

This may naturally be divided into :

I. Special anatomy, the object of which is the investigation of the construction, form, and arrangement of the organs of any individual animal.

2. Comparative anatomy, the object of which is a comparison of the organs of two or more animals, with a view to determining their points of resemblance or dissimilarity.

If the organs, however, are subjected to a further analysis, they can be resolved into simple structures, apparently homogeneous, to which the name tissue has been given - e. $g$., epithelial, connective, muscle, and nerve tissue. When the tissues are subjected to a micruscopic analysis, it is found that they are not homogeneous in structure, but composed of still 
simpler elements, termed cells and fibers. The investigation of the internal structure of the organs, the physical properties and structure of the tissues, as well as, the structure of their component elements, the cells and fibers, constitutes a department of anatomic science known as HISTOLOGY, or as it is prosecuted largely with the microscope, Microscopic ANatomy.

Human anatomy is that department of anatomic science which has for its object the investigation of the construction of the human body.

\section{GENERAL STRUCTURE OF THE ANIMAL BODY.}

The body of every animal, from fish to man, may be divided into-

I. An axial and

2. An appendicular portion. The axial portion consists of the head, neck, and trunk; the appendicular portion consists of the anterior and posterior limbs or extremities.

The axial portion of all mammals, to which class man zoölogically belongs, as well as of all birds, reptiles, amphibians, and fish, is characterized by the presence of a bony, segmented axis, which extends in a longitudinal direction from before backward, and which is known as the vertebral column or backbone. In virtue of the existence of this column all the classes of animals just mentioned form one great division of the animal kingdom, the Vertebrata.

Each segment, or vertebra, of this axis consists of -

1. A solid portion, known as the body or centrum, and

2. A bony arch arising from the dorsal aspect and surmounted by a spinelike process.

At the anterior extremity of the body of the animal the vertebre are variously modified and expanded, and, with the addition of new elements, form the skull ; at the posterior extrenity they rapidly diminish in size, and terminate in man in a short, tail-like process. In many animals, however, the vertebral column extends for a considerable distance beyond the trunk into the tail. The vertebral column may be regarded as the foundation element in the plan of organization of all the higher animals and the center around which the rest of the body is developed and arranged with a certain degree of conformity. In all vertebrate animals the bodies of the segments of the vertebral column form a partition which serves to divide the trunk of the body into two cavities - viz., the dorsal and the ventral. 


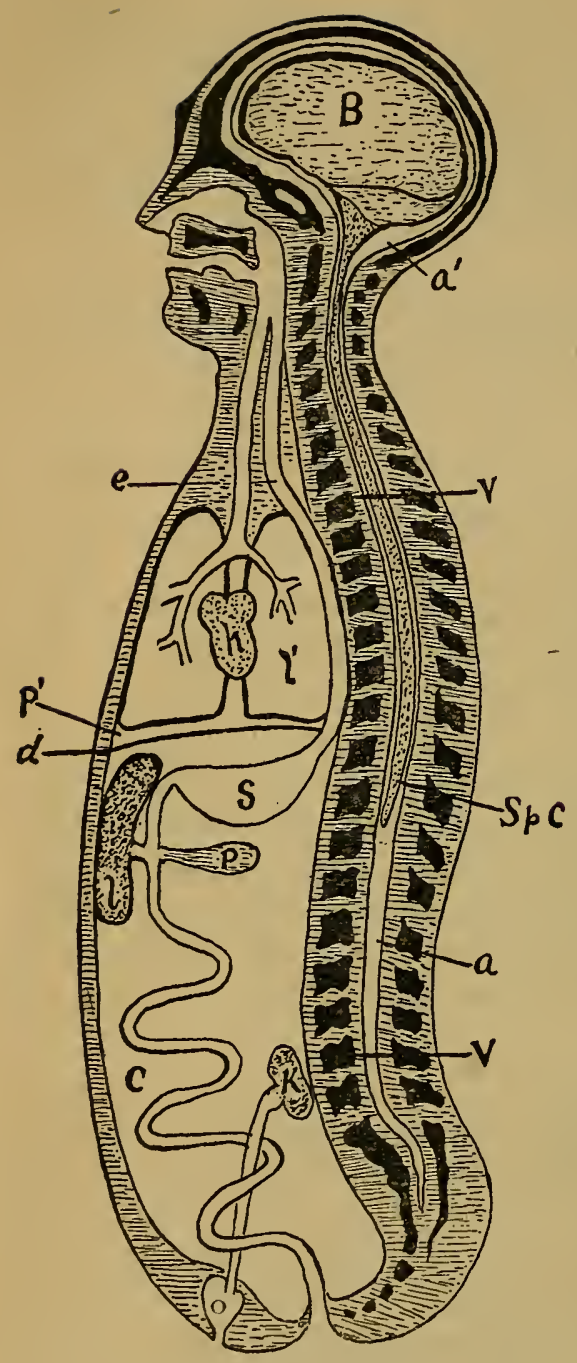

Fig. I.-Diagrammatic Longitudinal Section of the Bonyr

V, V. Bodies of the vertebræ which divide the body into the dorsal and ventral cavities. $a, a^{\prime}$. The dorsal cavity. C, $p^{\prime}$. The abdominal and thoracic divisions of the ventral cavity, separated from each other by a transverse muscular partition, the diaphragm $d$. B. The brain. Sp. C. The spinal cord. $e$. The esophagus. S. The stomach, from which continues the intestine to the opening at the posterior portion of the body. $l$. The liver. $p$. The pancreas. $k$. The kidney. $o$. The bladder. $l \prime$. The lungs. $h$. The heart.
The dorsal cavity is found not only in the trunk, but also in the head. It walls are formed partly by the arches which arise from the posterior or dorsal surface of the vertebræ and partly by the bones of the skull. If a longitudinal section be made through the center of the vertebral column, and including the head, the dorsal cavity will be observed running through its entire extent. (See Fig. I.) Though for the most part it is quite narrow, at the anterior extremity it is enlarged and forms the cavity of the skull. This cavity is lined by a membranous canal, the neural canal, in which is contained the brain "and the neural or spinal cord. Through openings in the sides of the dorsal cavity nerves pass out which connect the brain and spinal cord with all the structures of the body.

The ventral cavity is confined mainly to the trunk of the - body. Its walls are formed by muscles and skin, strengthened in most animals by bony arches, the ribs. Within the ventral cavity is contained a musculo-membranous tube or canal known as the alimentary or food canal, which begins at the mouth on the ventral side of the head, and, after passing through the neck and trunk, terminates at the posterior extremity of the 
trunk at the anus. It may be divided into mouth, pharynx, esophagus, stomach, small and large intestines.

In all mammals the ventral cavity is divided by a musculo-membranous partition into two smaller cavities, the thorax and abdomen. The former contains the lungs, heart and its great blood-vessels, and the anterior part of the alimentary canal, the gullet or esophagus; the latter contains the continuation of the alimentary canal-that is, the stomach and intestinesand the glands in connection with it, the liver and pancreas. In the posterior portion of the abdominal cavity are found the kidneys, ureters, and bladder, and in the female the organs of reproduction. The thoracic and abdominal cavities are each lined by a thin serous membrane, known, respectively, as the pleural and peritoneal membranes, which, in addition, are reflected over the surfaces of the organs contained within them. The alimentary canal and the various cavities connected with it are lined throughout by a mucous membrane. The surface of the body is covered by the skin. This is composed of an inner portion, the derma, and an outer portion, the epidermis. The former consists of fibers, blood-vessels, nerves, etc.; the latter of layers of scales or cells. Embedded within the skin are numbers of glands, which exude, in the different classes of animals, sweat, oily matter, etc. Projecting from the surface of the skin are hairs, bristles, feathers, claws. Beneath the skin are found muscles, bones, blood-vessels, nerres, etc.

The appendicular portion of the body consists of two pairs of symmetric limbs, which project from the sides of the trunk, and which bear a determinate relation to the vertebral column. They consist fundamentally of bones, surrounded by muscles, blood-vessels, nerves and lymphatics. The limbs, though having a common plan of organization, are modified in form and adapted for prehension and locomotion in accordance with the needs of the animal.

Anatomic Systems.-All the organs of the body which have certain peculiarities of structure in common are classified by anatomists into systems - e. $g$., the bones, collectively, constitute the bony or osseous system; the muscles, the nerves, the skin, constitute, respectively, the muscular, the nervous, and the tegumentary systems.

Physiologic Apparatus.-More important from a physiologic point of view than a classification of organs based on similarities of structure is the natural association of two or more organs acting together for the accomplishment of some definite object, and to which the term physiologic apparatus has been applied. While in the community of organs which 
together constitute the animal body each one performs some definite function, and the harmonious coöperation of all is necessary to the life of the individual, everywhere it is found that two or more organs, though performing totally distinct functions, are coöperating for the accomplishment of some larger or compound function in which their individual functions are blended-e. g., the mouth, stomach, and intestines, with the glands connected with them, constitute the digestive apparatus, the object or function of which is the complete digestion of the food. The capillary bloodvessels and lymphatic vessels of the body, and especially those in relation to the villi of the small intestine, constitute the absorptive apparatus, the function of which is the introduction of new material into the blood. The heart and blood-vessels constitute the circulatory apparatus, the function of which is the distribution of blood to all portions of the body. The lungs and trachea, together with the diaphragm and the walls of the chest, constitute the respiratory apparatus, the function of which is the introduction of oxygen into the blood and the elimination from it of carbon dioxid and other injurious products. The kidneys, the ureters, and the bladder constitute the urinary apparatus. The skin, with its sweat-glands, constitutes the perspiratory apparatus, the functions of both being the excretion of waste products from the body. The liver, the pancreas, the mammary glands, as well as other glands, each form a secretory apparatus which elaborates some specific material necessary to the nutrition of the individual. The functions of these different physiologic apparatus-e. $g$., digestion, absorption of food, elaboration of blood, circulation of blood, respiration, production of heat, secretion, and excretion-are classified as nutritive functions, and have for their final object the preservation of the individual.

The nerves and muscles constitute the nervo-muscular apparatus, the function of which is the production of motion. The eye, the ear, the nose, the tongue, and the skin, with their related structures, constitute, respectively, the visual, auditory, olfactory, gustatory, and tactile apparatus, the function of which, as a whole, is the reception of impressions and the transmission of nerve impulses to the brain, where they give rise to visual, auditory, olfactory, gustatory, and tactile sensations.

The brain, in association with the sense organs, forms an apparatus related to mental processes. The larynx and its accessory organs--the lungs, trachea, respiratory muscles, the mouth and resonant cavities of the face-form the vocal and articulating apparatus, by means of which voice and articulate speech are produced. The functions exhibited by the apparatus just mentioned-viz., motion, sensation, language, mental and 
moral manifestations-are classified as functions of relation, as they serve to bring the individual into conscious relationship with the external world.

The ovaries and the testes are the essential reprorluctive organs, the former producing the germ-cell, the latter the spermatic element; together with their related structures, - the fallopian tubes, uterus, and vagina in the female, and the urogenital canal in the male,- they constitute the reproductive apparatus characteristic of the two sexes. Their coöperation results in the union of the germ-cell and spermatic element and the consequent development of a new being. The function of reproduction serves to perpetuate the species to which the individual belongs.

The aninial body is therefore not a homogeneous organism, but one composed of a large number of widely dissimilar but related organs. But as all vertebrate animals have the same general plan of organization, there is a marked similarity both in form and structure anong corresponding parts of different animals. Hence it is that in the study of human anatomy a knowledge of the form, construction, and arrangement of the organs in different types of animal life is essential to its correct interpretation; also it is that in the investigation and comprehension of the complex problems of human physiology a knowledge of the functions of the organs as they manifest themselves in the different types of animal life is indispensable. As many of the functions of the human body are not only complex, but the organs exhibiting them are practically inaccessible to investigation, we must supplement our knowledge and judge of their functions by analogy, by attributing to them, within certain limits, the functions revealed by experimentation upon the corresponding but simpler organs of lower animals. This experimental knowledge, corrected by a study of the clinical phenomena of disease and the results of post-mortem investigations, forms the basis of modern human physiology.

\section{CHEMIC COMPOSITION OF THE HUMAN BODY.}

Since it has been demonstrated that every exhibition of functional activity is associated with changes of structure, it has been apparent that a knowledge of the chemic composition of the body, not only when in a state of rest, but to a far greater degree when in a state of activity, is necessary to a correct understanding of the intimate nature of physiologic processes. Though the analysis of the dead body is comparatively easy, the determina- 
tion of the successive changes in composition of the living body is attended with many difficulties. The living material, the bioplasm, is not only complex and unstable in composition, but extremely sensitive to all physical and chemic influences. The methods, therefore, which are employed for analysis destroy its composition and vitality, and the products which are obtained are peculiar to dead rather than to living material.

Chemic analysis, therefore, may be directed-

I. To the determination of the composition of the dead body.

2. To the determination of the successive changes in composition which the living bioplasm undergoes during functional activity.

A chemic analysis of the dead body, with a view to disclosing the substances of which it is composed, their properties, their intimate structure, their relationship to one another, constitutes what might be termed CHEMIC ANATOMY. An investigation of the living material and of the successive changes it undergoes in the performance of its functions constitutes what has been termed Chemic Physiology or Physiologic Chemistry.

By chemic analysis the animal body can be reduced to a number of liquid and solid compounds which belong to both the inorganic and organic worlds. These compounds, resulting from a proximate analysis, have been termed proximate principles. That they may merit this term, however, they must be obtained in the form under which they exist in the living condition. The organic compounds consist of representatives of the carbohydrate, fatty, and proteid groups of organic bodies; the inorganic compounds consist of water, various acids, and inorganic salts.

The compounds or proximate principles thus obtained can be further resolved by an ultimate analysis into a small number of chemic elements which are identical with elements found in many other organic as well as inorganic compounds. The different chemic elements which are thus obtained, and the percentage in which they exist in the body, are as follows -viz., oxygen, 72 per cent.; hydrogen, 9.10 ;-nitrogen, 2.5 ; carbon, I3.50; phosphorus, 1.15; calcium, 1.30; sulphur, 0.147; sodium, 0.10 ; potassium, 0.026 ; chlorin, 0.085 ; fluorin, iron silicon, magnesium, in small and variable amounts.

\section{THE CARBOHYDRATES.}

The carbohydrates constitute a group of organic bodies, consisting mainly of starches and sugars, having their origin for the most part in the vegetable world. In many respects they are closely related, and by appropriate means are readily converted into one another. In composition 
they consist of the elements carbon, hydrogen, and oxygen. As their name implies, the hydrogen and oxygen are present in the majority of these compounds in the proportion to form water, or as $2: \mathrm{r}$. JThe molecule of the carbohydrates just mentioned consists of either six atoms of carbon or a multiple of six; in the latter case the quantity of hydrogen and oxygen taken up by the carbon is increased, though the ratio remains unchanged.

The carbohydrates may be divided into three groups-viz. : (I) Amyloses, including starch, dextrin, glycogen, and cellulose; (2) dextroses, including dextrose, levulose, galactose; (3) saccharoses, including saccharose, lactose, and maltose. According to the number of carbon atoms entering into the second group (six), they are frequently termed monosaccharids; those of the third group, disaccharids-twice six; those of the first group, polysaccharids-multiples of six.

Though but few of the members of the carbohydrate group are constituents of the human body, yet on account of their importance as foods, and their relation to one another, a few of their chemic features will be stated in this connection.

1. AMYLOSES, $\left(\mathrm{C}_{6} \mathrm{H}_{10} \mathrm{O}_{5}\right)_{n}$.

Starch is widely distributed in the vegetable world, being abundant in the seeds of the cereals, leguminous plants, and in the tubers and roots of some vegetables. It occurs in the form of microscopic granules, which vary in size, shape, and appearance, according to the plant from which they are obtained. Each granule presents a nucleus, or hilum, around which is arranged a series of eccentric rings, alternately light and dark. The granule consists of an envelope and stroma of cellulose, containing in its meshes the true starch material-granulose. Starch is insoluble in cold water and alcohol. When heated with water up to $70^{\circ} \mathrm{C}$., the granules swell, rupture, and liberate the granulose, which forms an apparent solution; if present in sufficient quantity, it forms a gelatinous mass termed starch paste. On the addition of iodin, starch strikes a characteristic deep blue color ; the compound formed-iodid of starch-is weak, and the color disappears on heating, but reappears on cooling.

Boiling starch with dilute sulphuric acid (twenty-five per cent.) converts it into dextrose. In the presence of vegetable diastase or animal ferments, starch is converted into maltose and dextrose, two forms of sugar.

Dextrin is a substance formed as an intermediate product in the transformation of starch into sugar. There are at least two principal varietieserythrodextrin, which strikes a red color with iodin, and aihrö̈dextrin, 
which is without color when treated with this reagent. In the pure state dextrin is a yellow-white powder, soluble in water. In the presence of animal ferments erythrodextrin is converted into maltose.

Glycogen is a constituent of the animal liver, and, to a slight extent, of muscles and of tissues generally. In the tissues of the embryo it is especially abundant. When obtained in a pure state it is an amorphous, white powder. It is soluble in water, forming an opalescent solution. With iodin it strikes a port-wine color. In some respects it resembles starch, in others dextrin. Like vegetable starch, glycogen or animal starch can be converted by dilute acids and ferments into sugar (maltose).

Cellulose is the basis material of the more or less solid framework of plants. It is soluble only in an ammoniacal solution of cupric oxid, from which it can be precipitated by acids. It is an amorphous powder; dilute acids can convert it into dextrose.

2. DEXTROSES, $\mathrm{C}_{6} \mathrm{H}_{12} \mathrm{O}_{6}$.

Dextrose, glucose, or grape-sugar is found in grapes, most sweet fruits, and honey, and as a normal constituent of liver, blood, muscles, and other animal tissues. In the disease diabetes mellitus it is found also in the urine.

When obtained from any source, it is soluble in water and in hot alcohol, from which it crystallizes in six-sided tables or prisms. As usually met with, it is in the form of irregular, warty masses. It is sweet to the taste ; less so, however, than cane sugar. It is dextro-rotatory, turning the plane of polarized light to the right. In alkaline solutions dextrose absorbs oxygen, and hence in the presence of metallic salts, copper, bismuth, silver, etc., it acts as a reducing agent. On this property the various tests for dextrose, as well as other sugars which have the same property, are based.

Fehling's Test.-The solution usually employed for both qualitative and quantitative purposes is a solution of cupric hydroxid made alkaline by an excess of sodium or potassium hydroxid, with the addition of sodium and potassium tartrate. This solution, originally suggested by Fehling, bears his name. It is made by dissolving cupric sulphate 34.64 grams, potassium hydroxid 125 grams, sodium and potassium tartrate 173 grams in I liter of distilled water.

The reaction is expressed by the following equation :

$$
\mathrm{CuSO}_{4}+2 \mathrm{KOH}=\mathrm{Cu}(\mathrm{OH})_{2}+\mathrm{K}_{2} \mathrm{SO}_{4} \text {. }
$$


The object of the sodiun and potassium tartrate is to hold the $\mathrm{Cu}(\mathrm{OH})_{2}$ in solution. If a few cubic centimeters of this deep blue solution be boiled and dextrose then added and the solution again heated to the boiling-point, the cupric hydroxid is reduced to the condition of a cuprous oxid, which shows itself as a red or orange-yellow precipitate. The color of the precipitate depends on the relative excess of either copper or sugar, being red with the former, orange or yellow with the latter. The delicacy of this test is shown by the fact that a few minims of this solution will detect in one c.c. of water the $\frac{1}{15}$ of a milligram of sugar.

For quantitative analysis, ten c.c. of Fehling's solution, diluted with forty c.c. of water, are heated in a porcelain capsule, to which the dextrose solution is cautiously added from a buret until the blue color entirely disappears. The strength of this solution is such that one c.c. is decolorized by five milligrams of sugar, from which the percentage of sugar in any solution can be determined.

Fermentation Test.- If to a solution of dextrose a small quantity of the yeast plant be added, and the solution kept at a temperature of $25^{\circ} \mathrm{C}$., it will gradually undergo fermentation; that is, will be reduced to simpler compounds and especially to alcohol and carbon dioxid. The change expressed in the following equation :

$$
\underset{\text { Dextrose. Alcohol. }}{\mathrm{C}_{6} \mathrm{H}_{12} \mathrm{O}_{6}=} \begin{array}{r}
{ }_{2} \mathrm{C}_{2} \mathrm{H}_{6} \mathrm{O} \\
\text { Carbon } \\
2 \mathrm{CO}_{2} \\
\text { Dioxid. }
\end{array}
$$

About ninety-five per cent. of the dextrose is so changed, the remaining five per cent. yielding secondary products-succinic acid, glycerin, etc.

Levulose, or fruit-sugar, is found in association with dextrose as a constituent of many fruits. It is sweeter than dextrose and more soluble in both water and dilute alcohol. From alcoholic solutions it crystallizes in fine, silky needles, though it usually occurs in the form of a syrup.

Levulose is distinguished from dextrose by its property of turning the plane of polarized light to the left; the extent to which it does so, however, varies with the temperature and concentration of the solution.

Under the influence of the yeast plant it slowly undergoes fermentation, yielding the same products as dextrose. It also has a reducing action on cupric oxid.

Galactose is obtained by boiling milk-sugar (lactose) with dilute sulphuric acid. In many chemic relations it resembles dextrose. It is less soluble in water, however, crystallizes more easily, and has a greater dextro-rotatory power. It also undergoes fermentation with the yeast plant. 
3. SACCHAROSES, $\mathrm{C}_{12} \mathrm{H}_{22} \mathrm{O}_{11}$.

Saccharose, or cane-sugar, is widely distributed throughout the vegetable world, but is especially abundant in sugar-cane, sorghum cane, sugarbeet, Indian corn, etc. It crystallizes in large monoclinic prisms. It is soluble in water and in dilute alcohol. Saccharose has no reducing power on cupric oxid, and hence its presence can not be detected by Fehling's solution. It is dextro-rotatory. Boiled with dilute mineral, as well as organic acids, saccharose combines with water, and undergoes some change in virtue of which it rotates the plane of polarized light to the left, and hence the product is termed invert sugar. This latter has been shown to be a mixture of equal quantities of levulose and dextrose. This inversion of saccharose through hydration and decomposition is expressed by the following equation :

$$
\begin{aligned}
& \mathrm{C}_{12} \mathrm{H}_{22} \mathrm{O}_{11}+\mathrm{H}_{2} \mathrm{O}=\mathrm{C}_{6} \mathrm{H}_{12} \mathrm{O}_{6}+\mathrm{C}_{6} \mathrm{H}_{12} \mathrm{O}_{6} \\
& \text { Saccharose. Water. }
\end{aligned}
$$

Saccharose is not directly fermentable by yeast, but through the specific action of a ferment, invertin or invertase, secreted by the yeast plant, or the inverting ferment of the small intestine, it undergoes inversion, as previously stated, after which it is readily fermented, yielding alcohol and carbon dioxid.

Lactose is the form of sugar found exclusively in the milk of the mammalia, from which it can be obtained in the form of hard, white, rhombic prisms united with one molecule of water. It is soluble in water, insoluble in alcohol and ether. It is dextro-rotatory. It reduces cupric oxid, but to a less extent than dextrose. Dilute acids decompose it into equal quantities of dextrose and galactose. Lactose is not fermentable with yeast, but in the presence of the lactic acid bacillus it is decomposed into lactic acid, and finally into butyric acid, as follows :

$$
\begin{gathered}
\mathrm{C}_{12} \mathrm{H}_{22} \mathrm{O}_{11}+\mathrm{H}_{2} \mathrm{O}={ }_{4} \mathrm{C}_{3} \mathrm{H}_{6} \mathrm{O}_{3} \\
\text { Lactose. } \\
{ }_{2} \mathrm{C}_{3} \mathrm{H}_{6} \mathrm{O}_{3}=\underset{\text { Water. Lactic Acid. }}{\text { Lactic Acid. }}=\underset{\text { Butyric Acid. }}{\mathrm{C}_{4} \mathrm{H}_{8} \mathrm{O}_{2}}+\underset{\begin{array}{c}
\text { Carbon } \\
\text { Dioxid. }
\end{array}}{2 \mathrm{CO}_{2}}+\underset{2}{2 \mathrm{H}_{2}} \begin{array}{c}
\text { Free } \\
\text { Hydrogen. }
\end{array}
\end{gathered}
$$

Maltose is a transformation product of starch, and arises whenever the latter is acted on by malt extract or the diastatic ferments in saliva and pancreatic juice. It can also be produced by the action of dilute sulphuric acid on starch. The change is expressed by the following equation :

$$
\begin{gathered}
2 \mathrm{C}_{6} \mathrm{H}_{10} \mathrm{O}_{5}+\mathrm{H}_{2} \mathrm{O}=\mathrm{C}_{12} \mathrm{H}_{22} \mathrm{O}_{11} \\
\text { Starch. } \\
\text { Water. }
\end{gathered}
$$


Maltose crystallizes in the forn of white needles, which are soluble in water and in dilute alcohol. It is dextro-rotatory. In the presence of ferments and dilute acids maltose undergoes hydration and decomposition, giving rise to two molecules of dextrose. It has a reducing action on cupric oxid. Fermentation is readily caused by yeast, but whether directly or indirectly by inversion is somewhat uncertain.

O sazones.-All the sugars which possess the power of reducing cupric oxid are capable of combining with phenyl-hydrazin, with the formation of compounds termed osazones. The osazones so formed are crystalline in structure, but have different melting-points, varying degrees of solubility and optic properties, all of which serve to detect the various sugars and to distinguish one from the other. Of the different osazones, phenyl-glucosazone is the most characteristic, and occurs in the form of long, yellow needles. It may be obtained from dextrose by the following method: To fifty c.c. of a dextrose solution add two gm. of phenyl-hydrazin and two gm. of sodium acetate, and boil for an hour. On cooling, the osazone crystallizes in the form of long, yellow needles.

\section{THE FATS.}

The fats constitute a group of organic bodies found in the tissues of both vegetables and animals. In the vegetable world they are largely found in fruits, seeds, and nuts, where they probably originate from a transformation of the carbohydrates. In the animal body the fats are found largely in the subcutaneous tissue, in the marrow of bones, in and around various internal organs and in milk. In these situations fat is contained in small, round or polygon-shaped vesicles, which are united by areolar tissue and surrounded by blood-vessels. At the temperature of the body the fat is liquid, but after death it soon solidifies from the loss of heat.

The fats are compounds consisting of carbon, hydrogen, and oxygen, of which the first is the chief ingredient, forming by weight about seventy-five per cent., while the last is present only in small quantity. The fat, as found in animals, is a mixture, in varying proportions in different animals, of three neutral fats-stearin, palmitin, and olein. Each fat is a derivative of glycerin and the particular acid indicated by its name-e.g., stearic acid, in the case of stearin, etc. The reaction which takes place in the combination of glycerin and the acid is expressed in the following equation :

$$
\begin{aligned}
& \mathrm{C}_{3} \mathrm{H}_{5}(\mathrm{HO})_{3}+\left(\mathrm{HC}_{18} \mathrm{H}_{35} \mathrm{O}_{2}\right)_{3}=\mathrm{C}_{3} \mathrm{H}_{5}\left(\mathrm{C}_{18} \mathrm{H}_{35} \mathrm{O}_{2}\right)_{3}+{ }_{3} \mathrm{H}_{2} \mathrm{O} \text {. } \\
& \text { Glycerin. Stearic Acid. Stearin, Water, }
\end{aligned}
$$


Hence, strictly speaking, the fats are compound ethers, in which the hydrogen of the organic acid is replaced by the trivalent radicle, tritenyl, $\mathrm{C}_{3} \mathrm{H}_{5}$.

Stearin, $\mathrm{C}_{3} \mathrm{H}_{5}\left(\mathrm{C}_{18} \mathrm{H}_{35} \mathrm{O}_{2}\right)_{3}$, is the chief constituent of the more solid fats. It is solid at ordinary temperatures, melting at $55^{\circ} \mathrm{C}$., then solidifying again as the temperature rises, until at $7 \mathrm{r}^{\circ} \mathrm{C}$. it melts permanently. It crystallizes in square tables.

Palmitin, $\mathrm{C}_{3} \mathrm{H}_{5}\left(\mathrm{C}_{16} \mathrm{H}_{31} \mathrm{O}_{2}\right)_{3}$, is a semifluid fat, solid at $45^{\circ} \mathrm{C}$. and melting at $62^{\circ} \mathrm{C}$. It crystallizes in fine needles, and is soluble in ether.

Olein, $\mathrm{C}_{3} \mathrm{H}_{5}\left(\mathrm{C}_{18} \mathrm{H}_{33} \mathrm{O}_{2}\right)_{3}$, is a colorless, transparent fluid, liquid at ordinary temperatures, only solidifying at $0^{\circ} \mathrm{C}$. It possesses marked solvent powers, and holds stearin and palmitin in solution at the temperature of the body.

Saponification.-When subjected to the action of superheated steam, a neutral fat is saponified-i.e., decomposed into glycerin and the particular acid indicated by the name of the fat used : e.g., stearic, palmitic, or oleic. The reaction is expressed as follows :

$$
\underset{\text { Olein. }}{\mathrm{C}_{3} \mathrm{H}_{5}}\left(\mathrm{C}_{18} \mathrm{H}_{33} \mathrm{O}_{2}\right)_{3}+\underset{\text { Water. }}{3 \mathrm{H}_{2} \mathrm{O}}=\underset{\text { Glycerin. }}{\mathrm{C}_{3} \mathrm{H}_{5}(\mathrm{HO})_{3}}+\underset{\text { Oleic Acid. }}{3\left(\mathrm{C}_{18} \mathrm{H}_{34} \mathrm{O}_{2}\right)}
$$

The fatty acids thus obtained are characterized by certain chemic features, as follows :

Stearic acid is a firm, white solid, fusible at $69^{\circ} \mathrm{C}$. It is soluble in ether and alcohol, but not in water.

Palmitic acid occurs in the form of white, glistening scales or needles, melting at $62^{\circ} \mathrm{C}$.

Oleic acid is a clear, colorless liquid, tasteless and odorless when pure. It crystallizes in white needles at $0^{\circ} \mathrm{C}$.

If this saponification take place in the presence of an alkali, - e. $g$., potassium hydroxid, sodium hydroxid,-the acid produced combines at once with the alkali to form a salt known as a soap, while the glycerin remains in solution. The reaction is as follows :

$$
\begin{aligned}
& 3 \mathrm{KHO}+\left(\mathrm{C}_{18} \mathrm{H}_{34} \mathrm{O}_{2}\right)_{3}=3\left(\mathrm{KC}_{18} \mathrm{H}_{33} \mathrm{O}_{2}\right)+\mathrm{C}_{3} \mathrm{H}_{5}(\mathrm{HO})_{3} \\
& \text { Potassium. Oleic Acid. Potassium Oleate. Glycerin. }
\end{aligned}
$$

All soaps are, therefore, salts formed by the union of alkalies and fatty acids. The sodium soaps are generally hard, while the potassium soaps are soft. Those made with stearin and palmitin are harder than those made with olein. If the soap is composed of lead, zinc, copper, etc., it is insoluble in water. 
Emulcification.--When a neutral oil is vigorously shaken with water or other fluid, it is broken up into minute globules that are more or less permanently suspended; the permanency depending on the nature of the liquid. The most permanent emulsions are those made with soap solutions. The process of emulsification and the part played by soap, can be readily observed by placing on a few cubic centimeters of a solution of sodium carbonate 0.25 per cent. of a small quantity of a perfectly neutral oil to which has been added 2 or 3 per cent. of a fatty acid. The combination of the acid and the alkali at once forms a soap. The energy set free by this combination rapidly divides up the oil into extremely minute globules. A spontaneous enulsion is thus formed.

In addition to the ordinary fats, there are present in different tissues several compounds which, though usually regarded as fats, nevertheless differ materially from them in composition, containing, as they do, both nitrogen and phosphorus. These nitrogenized or phosphorized fats are as follows :

Lecithin, $\mathrm{C}_{44} \mathrm{H}_{90} \mathrm{~N} . \mathrm{PO}_{9}$, is found in blood, lymph, red and white corpuscles, nerve tissue, yolk of eggs, etc. When pure, it presents itself generally under the form of a white, crystalline powder, though sometimes as a white, waxy mass. Lecithin is easily decomposed, yielding, with various reagents, glycero-phosphoric acid, cholin and stearic acid.

Protagon, $\mathrm{C}_{160} \mathrm{H}_{303} \mathrm{~N}_{5} \mathrm{PO}_{35}$, is found most abundantly in the brain tissue, especially in the white portion. It crystallizes from warm alcoholic solutions, on cooling, in the form of white needles, generally arranged in groups. It melts at $200^{\circ} \mathrm{C}$, and forms a syrupy liquid.

Cerebrin, $\mathrm{C}_{17} \mathrm{H}_{33} \mathrm{~N} . \mathrm{O}_{3}$, is found largely in the brain, in nerves, and in pus-corpuscles. It is a soft, white, amorphous powder, insoluble in water, but swelling up like starch in boiling water. When boiled with dilute acids, it is decomposed, yielding a fermentable dextro-rotatory sugar, identical with galactose. Cerebrin may, therefore, be regarded as a glucosid.

\section{THE PROTEIDS.}

The proteids constitute a group of organic bodies which are found in both vegetable and animal tissues. Though present in all animal tissues, they are especially abundant in muscles and bones, where they constitute twenty per cent. and thirty per cent. respectively. Though genetically related, and possessing many features in common, the different members of the proteid group are distinguished by characteristic physical and chemic properties. 
The average percentage composition of several proteids is shown in the following analyses :

C. H. N. O. S.

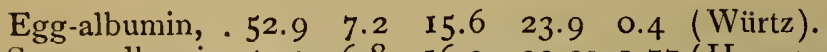

Serum-albumin, 53.0 6.8 16.0 22.29 1.77 (Hammersten).

Casein,. . . 53.37 .07 I5.9I 22.03 0.82 (Chittenden and Painter). Myosin, . . $52 . \$_{2} 7.1$ I 16.7721 .901 .27 (Chittenden and Cummins).

The molecular composition of the proteids is not definitely known, and the formula which have been suggested are therefore only approximative. Leow assigns to albumin the formula $\mathrm{C}_{72} \mathrm{H}_{112} \mathrm{~N}_{18} \mathrm{O}_{22} \mathrm{~S}$, while Schützenberger raises the numbers to $\mathrm{C}_{240} \mathrm{H}_{392} \mathrm{~N}_{65} \mathrm{O}_{75} \mathrm{~S}_{3}$, either of which shows that the proteid molecule is extremely complex. As a class, the proteids are characterized by the following properties :

I. Indiffusibility.-None of the proteids normally assumes the crystalline form, and hence they are not capable of diffusing through parchment or an animal membrane. Peptone, a product of the digestion of proteids, is an exception as regards its diffusibility. As met with in the body, all proteids are amorphous, but vary in consistence from the liquid to the solid state. The colloid character of the proteids permits of their separation and purification from crystalloid diffusible compounds by the process of dialysis.

2. Solubility.- Some of the proteids are soluble in water, others in solutions of the neutral salts of varying degrees of concentration, in strong acids and alkalies. All are insoluble in alcohol and ether.

3. Coagulability.-Under the influence of heat and various acids and animal ferments, the proteids readily pass from the soluble liquid state to the insoluble solid state, attended by a permanent alteration in their chemic composition. To this change the term coagulation has been given. The various proteids not only coagulate at different temperature's, but with different chemic reagents-distinctive features which permit not only of their detection, but separation. Proteids are capable of precipitation without losing their solubility by ammonium sulphate, sodium chlorid and magnesium sulphate.

4. Fermentability.-In the presence of specific microörganisms-bacteria-the proteids, owing to their complexity and instability, are prone to undergo disintegration and reduction to simpler compounds. This decomposition or putrefaction occurs most readily when the conditions most favorable to the growth of bacteria are present-viz., a temperature varying from $25^{\circ} \mathrm{C}$, to $40^{\circ} \mathrm{C}$., moisture and oxygen, The intermediate 
as well as the terminal products of the decomposition of the proteids are numerous, and vary with the composition of the proteid and the specific physiologic action of the bacteria. Among the intermediate products is a series of alkaloid bodies, some of which possess marked toxic properties, known as ptomains or toxins. The toxic symptoms which frequently follow the ingestion of foods in various stages of putrefaction are to be attributed to these compounds. The terminal products are represented by hydrogen sulphid, ammonia, carbon dioxid, fats, phosphates, nitrates, etc.

Color Tests for Proieids.-When proteids are present in solution, they may be detected by the following color reactions-viz. :

I. Xanthoproteic. The solution is boiled with nitric acid for several minutes, when the proteid assumes a light yellow color. After the solution has cooled, the addition of ammonia changes the color to an orange or amber-red.

2. The rose-red reaction. The solution is boiled with acid nitrate of mercury (Millon's reagent) for a few minutes, when the coagulated proteid turns a purple-red color.

3. The blue-violet reaction. A few drops of copper sulphate solution are first added to the proteid solution, and then an excess of sodium hydroxid. A blue-violet color is produced, which deepens somewhat on beating, but no further change ensues.

The proteids found in the animal body, though possessing many features in common, are nevertheless characterized by certain special features which not only serve for their identification, but for their classification into well-defined groups, as follows :

\section{NATIVE PROTEIDS.}

The members of this group are soluble in water, in dilute saline solutions, and in saturated solutions of sodium chlorid and magnesium sulphate. They are coagulated by heat, and when dried form an amber-colored mass.

(a) Serum-albumin is found in blood, lymph, chyle, tissue fluids, and milk. It is obtained readily by precipitation from blood-serum, after the other proteids have been removed, on the addition of ammonium sulphate. When freed from saline constituents, it presents itself as a pale, amorphous substance, soluble in water and in strong nitric acid. It is coagulated at a temperature of $73^{\circ} \mathrm{C}$, , as well as by various acids-e. $g$. , citric, picric, nitric, etc. It has a rotatory power of $-62.6^{\circ}$. 
(6) Egg-albumin.-Though not a constituent of the human body, egg-albumin resembles the foregoing in many respects. When obtained in the solid form from the white of the egg, it is a yellow mass without taste or odor. Though similar to serum-albumin, it differs from it in being precipitated by ether, in coagulating at $54^{\circ} \mathrm{C}$, , and in having a lower rotatory power, $-35 \cdot 5^{\circ}$.

\section{GLOBULINS.}

The nembers of this group are insoluble in water and in saturated solutions of sodium chlorid and magnesium sulphate and ammonium sulphate. They are soluble, however, in dilute saline solutions-e. g., sodium chlorid (one per cent.), potassium chlorid, ammonium chlorid, etc. They are coagulated by heat.

(a) Serum-globulin or Paraglobulin.-This proteid, as its name implies, is found in blood-serum, though it is present in other animal fluids. When precipitated by magnesium sulphate or carbon dioxid, it presents itself as a flocculent substance, insoluble in water, soluble in dilute acids and alkalies, and coagulating at $75^{\circ} \mathrm{C}$.

(b) Fibrinogen.-This proteid is found in blood plasma in association with serum-globulin and serum-albumin. It is also present in lymph-tissue fluids and in pathologic transudates. It can be obtained from blood-plasma which has been previously treated with magnesium sulphate on the addition of a saturated solution of sodium chlorid. It is soluble in dilute acids and alkalies, and coagulates at $5^{\circ} \mathrm{C}$.

(c) Myosinogen.-This proteid is a constituent of the protoplasm of the muscle-fibers. During the living condition it is liquid, but after death it readily undergoes decomposition into an insoluble portion known as myosin and a soluble albumin. It is soluble in dilute hydrochloric acid and dilute alkalies. It coagulates at $56^{\circ} \mathrm{C}$.

(d) Globin.-This is a product of the spontaneous decomposition of the coloring matter of the blood,-hemoglobin,-and arises when the latter is exposed to the air.

(e) Crystallin or Globulin.-This is obtained by passing a stream of $\mathrm{CO}_{2}$ through a watery extract of the crystalline lens.

\section{DERIVED ALBUMINS OR ALBUMINATES.}

The proteids of this group are derived from both native albumins and globulins by the gradual action of dilute acids and alkalies, and may be regarded as compounds of a proteid with an acid or an alkali. 
(a) Acid-albumin.-This is formed when a native albumin is digested with dilute hydrochloric acid (0.2 per cent.) or dilute sulphuric acid for some minutes. It is precipitated by neutralization with sodium hydroxid (o. I per cent. solution). After the precipitate is washed, it is found to be insoluble in distilled water and in neutral saline solutions. In acid solutions it is not coagulated by lieat.

(b) Alkali-albumin.-This is formed when a native albumin is treated with a dilute alkali-e.g., o. I per cent. of sodium hydroxid-for five or ten minutes. On careful neutralization with dilute hydrochloric acid, it is precipitated. It is also insoluble in distilled water and in alkaline solutions; it is not coagulable by heat.

(c) Caseinogen.-This is the principal proteid of milk, in which it exists in association with an alkali, and hence was formerly regarded as a native alkali-albumin. It is precipitated by acetic acid and by magnesium sulphate. It is coagzulated by rennet-that is, separated into an insoluble proteid, casein or tyrein, and a soluble albumin. Calcium phosphate seems to be the alkali necessary to this process, for if it be removed by dialysis, or precipitated by the addition of potassium oxalate, coagulation does not take place.

\section{COAGULATED PROTEIDS.}

Although these proteids are not found as constituents of the animal organism, they possess much interest on account of their relation to prepared foods and to the digestive process. They are produced when solutions of egg-albumin, serum-albumin, or globulins are subjected to a temperature of $100^{\circ} \mathrm{C}$. or to the prolonged action of alcohol. They are insoluble in water, in dilute acids, and in neutral saline solutions. In this same group may be included also those coagulated proteids which are produced by the action of animal ferments on soluble proteids $-e . g$, fibrin, myosin, casein.

Fibrin.-Fibrin is derived from a soluble proteid-fibrinogen-by the action of a special ferment. It is not present under normal circumstances in the circulating blood, but makes its appearance after the blood is withdrawn from the vessels and at the time of coagulation. It can also be obtained by whipping the blood with a bundle of twigs, on which it accumulates. When freed from blood by washing under water, it is seen to consist of bundles of white elastic fibers or threads. It is insoluble in water, in alcohol, and ether. In dilute acids it swells, becomes transparent, and finally is 
converted into acid-albumin. In dilute alkalies a similar change takes place, but the resulting product is an alkali-albumin. Fibrin possesses the property of decomposing hydrogen dioxid, $\mathrm{H}_{2} \mathrm{O}_{2}-i$. e., liberating oxygen, which accumulates in the form of bubbles on the fibrin. On incineration fibrin yields an ash which contains calcium phosphate and magnesium phosphate.

\section{PROTEOSES AND PEPTONES.}

During the progress of the digestive process, as it takes place in the stomach and intestines, there is produced by the action of the gastric and pancreatic juices, out of the proteids of the food, a series of new proteids, known as proteoses and peptones. The chemic properties of these substances will be considered in connection with the process of digestion.

\section{ALBUMINOIDS.}

The albuminoids constitute a group of substances similar to the proteids in many respects, though differing from them in others. When obtained from the tissues, in which they form an organic basis, they are found to be amorphous, colloid, and when decomposed yield products similar to those of the true proteids. The principal members of this group are as follows :

(a) Mucin.-This is the viscid, tenacious constituent of mucus secreted by the epithelial cells of mucous membranes. It is also present in the intercellular substance of the connective tissue. It is readily precipitated by acetic acid.

(b) Collagen, Ossein.-These are two closely allied, if not identical, substances, found respectively in the white fibrous connective tissue and in bone. When the tendons of muscles, the ligaments, or decalcified bone are boiled for several hours, the collagen and ossein are converted into soluble gelatin, which, when the solution cools, becomes solid.

(c) Chondrinigen. - This is supposed to be the organic basis of the more permanent cartilages. When they are boiled, they yield a substance which gelatinizes on cooling, and to which the name chondrin has been given.

(d) Elastin is the name given to the substance composing the fibers of the yellow, elastic connective tissue.

(e) Keratin is the substance found in all horny and epidermic tissues, such as hairs, nails, sçales, etc. It differs from most proteids in containing,a high percentage of "sulphur. 
( $f$ ) Nuclein.-This is an amorphous substance obtained from the nuclei of both animal and vegetable cells. The chief constituent appears to be nucleic acid. Chemic analysis shows that nuclein contains not less than three per cent. of phosphorus. On boiling with strong alkalies, nuclein is partially decomposed, yielding a proteid resembling globulin and phosphoric acid. A nucleoproteid is a compound of nuclein and a proteid.

\section{INORGANIC CONSTITUENTS.}

The inorganic compounds and mineral constituents obtained from the solids and fluids of the body are very numerous, and, in some instances, quite abundant. Though many of the compounds thus obtained are undoubtedly derivatives of the tissues and necessary to their physical and physiologic activity, others, in all probability, are decomposition products, or transitory constituents introduced with the food. Of the inorganic compounds, the following are the most important:

\section{WATER.}

Water is the most important of the inorganic constituents, as it is indispensable to life. It is present in all the tissues and fluids without exception, varying from 99 per cent. in the saliva to 80 per cent. in the blood, 75 per cent. in the muscles to 2 per cent. in the enamel of the teeth. The total quantity contained in a body weighing 75 kilograms ( 165 pounds) is 52.5 kilograms (II5 pounds). Much of the water exists in a free condition, and forms the chief part of the fluids, giving to them their characteristic degree of fluidity. Possessing the capability of holding in solution a large number of inorganic as well as some organic compounds, and being at the same time diffusible, it renders an interchange of materials between all portions of the body possible. It aids in the absorption of new material into the blood and tissues, and at the same time it transfers waste products from the tissues to the blood, from which they are finally eliminated, along with the water in which they are dissolved. A portion of the water is chemically combined with other tissue constituents, and gives to the tissues their characteristic physical properties. The consistency, elasticity, and pliability are, to a large extent, conditioned by the amount of water they contain. The total quantity of water eliminated by the kidneys, lungs, and skin amounts to about three kilograms (61/2 pounds). 


\section{CALCIUM COMPOUNDS.}

Calcium phosphate, $\mathrm{Ca}_{3}\left(\mathrm{PO}_{4}\right)_{2}$, has a very extensive distribution throughout the body. It exists largely in the bones, teeth, and to a slight extent in cartilage, blood, and other tissues. Milk contains 0.27 per cent. The solidity of the bones and teeth is almost entirely due to the presence of this salt, and is, therefore, to be regarded as necessary to their structure. It enters into chemic union with the organic matter, as shown by the fact that it can not be separated from it except by chemic means, such as hydrochloric acid. Though insoluble in water, it is held in solution in the blood and milk by the proteid constituents, and in the urine by the acid phosphate of soda. The total quantity of calcium phosphate which enters into the formation of the body has been estimated at 2.5 kilograms. The amount eliminated daily from the body has been estimated at $0.4 \mathrm{gm}$., a fact which indicates that nutritive changes do not take place with much rapidity in those tissues in which it is contained.

Calcium carbonate, $\mathrm{CaCO}_{3}$, is present in practically the same situations in the body as the phosphate, and plays essentially the same rôle. It is, however, found in the crystalline form, aggregated in small masses in the internal ear, forming the otoliths, or ear stones. Though insoluble, it is held in solution by the carbonic acid diffused through the fluids.

Calcium fluorid, $\mathrm{CaF}_{2}$, is found in bones and teeth.

\section{SODIUM COMPOUNDS.}

Sodium chlorid, $\mathrm{NaCl}$, is present in all the tissues and fluids of the body, but especially in the blood, 0.6 per cent., lymph,'0.5, and pancreatic juice, 0.25 per cent. The entire quantity in the body has been estimated at about $200 \mathrm{gm}$. Sodium chlorid is of much importance in the body, as it determines and regulates to a large extent the phenomena of diffusion which are there constantly taking place. This is illustrated by the fact that a solution of albumin placed in the rectum without the addition of this salt will not be absorbed. When the salt is added, absorption takes place. The ingested water is absorbed into the blood largely in consequence of the percentage of this salt which it contains. The normal percentage of sodium chlorid in the blood-plasma assists in maintaining the shape and structure of the red blood-corpuscles by determining the amount of water entering into their composition. The same is true of other tissue elements.

Sodium chlorid also influences the general nutritive process, increasing the disintegration of the proteids, as shown by the increased amount of urea excreted. During its existence in the body it undergoes some chemic 
transformations or decompositions, yielding its chlorid to form potassium chlorid of the blood-corpuscles and muscles and to form the hydrochloric acid of the gastric juice.

Sodium phosphate, $\mathrm{Na}_{2} \mathrm{IPO}_{4}$, is found in all solids and fluids of the body, to which, with but few exceptions, it imparts an alkaline reaction. This is especially true of blood, lymph, and tissue fluids generally. It is essential to physiologic action that all tissue elements should be bathed by an alkaline medium.

Sodium carbonate, $\mathrm{Na}_{2} \mathrm{CO}_{3}$, is generally found in association with the preceding salt. As it is also an alkaline compound, it assists in giving to the blood and lymph their characteristic alkalinity. In carnivorous animals the sodium phosphate is the more abundant, while in the herbivorous animals the reverse is true.

Sodium sulphate, $\mathrm{Na}_{2} \mathrm{SO}_{4}$, is present in many of the tissues and fluids, especially the urine. Though introduced in the food, it is also, in all probability, formed in the body from the decomposition and oxidation of the proteids.

\section{POTASSIUM COMPOUNDS.}

Potassium chlorid, $\mathrm{KCl}$, is met with in association with sodium chlorid in almost all situations in the body. It preponderates, however, in the tissue elements, especially in the muscle tissue, nerve tissue, and red corpuscles. The plasma with which these structures are bathed contains but a very small amount of this salt, but, as previously stated, a relatively large quantity of sodium chlorid. Though introduced to some extent in the food, it is very likely that it is also formed through the decomposition of the sodium chlorid.

Potassium phosphate, $\mathrm{K}_{2} \mathrm{HPO}_{4}$, is found in association with sodium phosphate in all the fluids and solids. As it has similar chemic properties, its functions are practically the same.

Potassium carbonate, $\mathrm{K}_{2} \mathrm{CO}_{3}$, is generally found with the preceding salt.

\section{MAGNESIUM COMPOUNDS.}

Magnesium phosphate, $\mathrm{Mg}_{3}\left(\mathrm{PO}_{4}\right)_{2}$, is found in all tissues, in association with calcium phosphate, though in much smaller quantity.

Magnesium carbonate, $\mathrm{MgCO}_{3}$, occurs only in traces in the blood.

Both of these compounds have functions similar to the calcium compounds, and exist, in all probability, under similar conditions. 


\section{IRON COMPOUNDS.}

Iron is a constituent of the coloring matter of the blood. Traces, however, are also found in lymph, bile, gastric juice, and in the pigment of the eyes, skin, and hair. The amount of iron contained in a body weighing seventy-five kilograms is about three gm. It exists under various formse. $g$., ferric oxid, ferrous oxid, and in combination with organic compounds.

Chemic analysis thus shows that the chemic elements into which the compounds may be resolved by an ultimate analysis do not exist in the body in a free state, but only in combination, and in characteristic proportions, to form compounds whose properties are the resultant of those of the elements. Of the four principal elements which make up ninety-seven per cent. of the body, $\mathrm{O}, \mathrm{H}, \mathrm{N}$ are extremely mobile, elastic, and possessed of great atomic heat. $\mathrm{C}, \mathrm{H}, \mathrm{N}$ are distinguished for the narrow range of their affinities, and for their chemic inertia. $\mathrm{C}$ possesses the great atomic cohesion. $\mathrm{O}$ is noted for the number and intensity of its combinations.

As the properties of the compounds formed by the union of elements must be the resultants of the properties of the elements themselves, it follows that the ternary compounds, starches, sugars, and fats must possess more or less inertia, and at the same time instability; while in the more complex proteids, in which sulphur and phosphorus are frequently combined with the four principal elemen!s, molecular instability attains its maximum. As all the foregoing compounds possess in varying degrees the properties of inertia and instability, it follows that living matter must possess corresponding properties, and the capability of undergoing unceasingly a series of chemic changes, both of composition and decomposition, in response to the chemic and physical influences by which it is, surrounded, and which underlie all the phenomena of life.

\section{PRINCIPLES OF DISSIMILATION.}

In addition to the previously mentioned compounds,-viz., carbohydrates, fats, proteids, and inorganic salts,--there is obtained by chemic analysis from the tissues and fluids of the body :

I. A number of organic acids, such as acetic, lactic, oxalic, butyric, propionic, etc., in combination with alkaline and earthy bases.

2. Organic compounds, such as alcohol, glycerin, cholesterin.

3. Pigments, such as those found in bile and urine.

4. Crystallizable nitrogenized bodies, such as urea, uric acid, xanthin, hippuric acid, creatin, creatinin, etc. 
While some few of these compounds may possibly be regarded as necessary to the physiologic integrity of the tissues and fluids, the majority of them are to be regarded as products of dissimilation of the tissues and foods in consequence of functional activity, and represent stages in their reduction to simpler forms previous to being eliminated from the body.

\section{PHYSIOLOGY OF THE CELL.}

A microscopic analysis of the tissues shows that they can be resolved into simpler elements, termed cells, which may, therefore, be regarded as the primary units of structure. Though cells vary considerably in shape, size, and chemic composition in the different tissues of the adult body, they are, nevertheless, descendants from typical cells, known as embryonic or undifferentiated cells, examples of which are the leukocytes of the blood and lymph and the first offspring of the fertilized ovum. Ascending the line of embryonic development, it will be found that every organized body originates in a single cell-the ovum. As the cell is the elementary unit of all tissues, the function of each tissue must be referred to the function of the cell. Hence the cell may be defined as the primary anatomic and physiologic unit of the organic world, to which every exhibition of life, whether normal or abnormal, is to be referred.

Structure of Cells.- Though cells vary in shape and size and internal structure in different portions of the body, a typical cell may be said to consist mainly of a gelatinous substance forming the body of the cell, termed protoplasm or hioplasm, in which is embedded a smaller spheric body, the mucleus. The shape of the adult cell varies according to the tissue in which it is found; when young and free to move in a fluid medium, the cell assumes a spheric form, but when subjected to pressure, may become cylindric, fusiform, polygonal, or stellate. Cells vary in size within wide limits, ranging from $\frac{1}{3 \frac{1}{2}}$ of an inch, the diameter of a red blood-corpuscle, to $\frac{1}{200}$ of an inch, the diameter of the large cells in the gray matter of the spinal cord. (See Fig. 2.)

The cell protoplasm consists of a soft, semifluid, gelatinous material, varying somewhat in appearance in different tissues. Though frequently homogeneous, it often exhibits a finely granular appearance under medium powers of the microscope. Young cells consist almost entirely of clear protoplasm. Mature cells contain, according to the tissue in which they are found, material of an entirely different character-e.g., small globules of fat, granules of glycogen, mucigen, pigments, digestive ferments, etc. 
Under high powers of the microscope the cell protoplasm is found to be pervaded by a network of fibers, termed spongioplasm, in the meshes of which is contained a clearer and more fluent substance, the hyaloplasm. The relative amount of these two constituents varies in different cells, the proportion of hyaloplasm being usually greater in young cells. The arrangement of the fibers forming the spongioplasm also varies, the fibers having sometimes a radial direction, in others a concentric disposition, but most frequently being distributed evenly in all directions. In many cells the outer portion of the cell protoplasm undergoes chemic changes and is transformed into a thin, transparent, homogeneous membrane,- the cell membrane,-which completely incloses the cell substance. The cell mem-

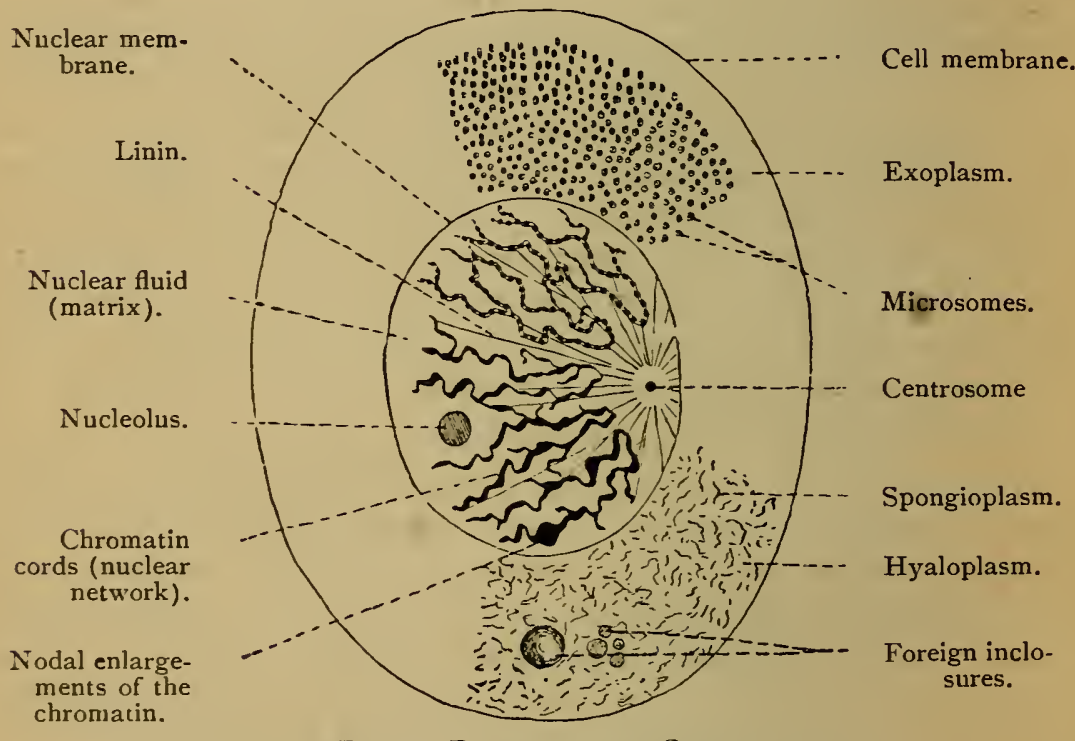

Fig. 2,-Diagram of a Cell.

Microsomes and spongioplasm are only partly drawn.

brane is permeable to water and watery solutions of various inorganic and organic substances. It is, however, not an essential part of the cell.

The nucleus is a small vesicular body embedded in the protoplasm near the center of the cell. In the resting condition of the cell it consists of a distinct membrane, composed of amphipyrenin, inclosing the nuclear contents. The latter consists of a homogeneous amorphous substance, - - the nuclear matrix, - in which is embedded the nuclear network. It can often be seen that a portion of one side of the nucleus, called the pole, is free from 
this network. The main cords of the network are arranged as V-shaped loops about it. These main cords send out secondary branches or twigs, which, uniting with one another, complete the network. The nuclear cords are composed of granules of chromatin,-so called because of its affinity for certain staining materials,-held together by an achromatin substance known as linin. Besides the nuclear network, there are embedded in the nuclear matrix one or more small bodies composed of pyrenin, known as nucleoli. At the pole of the nucleus, either within or just without in the protoplasm, is a small body, the centrosome, or pole corpuscle.

Chemic Composition of the Cell.-The composition of living protoplasm is difficult of determination, for the reason that all chemic and physical methods employed for its analysis destroy its vitality, and the products obtained are peculiar to dead rather than to living matter. Moreover, as protoplasm is the seat of constructive and destructive processes, it is not easy to determine whether the products of analysis are crude food constituents or cleavage or disintegration products. Nevertheless, chemic investigations have shown that even in the living condition protoplasm is a highly complex compound-the resultant of the intimate union of many different substances. About seventy-five per cent. of protoplasm consists of water and twenty-five per cent. of solids, of which the more important compounds are various nucleo-proteids (characterized by their large percentage of phosphorus), globulins, traces of lecithin, cholesterin, and frequently fat and carbohydrates. Inorganic salts, especially the potassium, sodium, and calcium chlorids and phosphates, are almost invariable and essential constituents.

\section{MANIFESTATIONS OF CELL LIFE.}

Growth, Nutrition.-All cells exhibit the three fundamental properties of life-viz., growth, nutrition, reproduction. All cells when newly reproduced are extremely small, but by the absorption of nutritive material from their surrounding medium, they gradually grow until they attain their mature size. 'This is accomplished by the power which living material possesses of transforming, vitalizing, and organizing crude nutritive material, through a series of upward changes, into material similar to itself. To all these changes the term assimilation, or anabolism, has been given. Some of the absorbed material, in all probability, never becomes an integral part of the living bioplasm, but undergoes disruption and oxidation, giving rise at once to heat and force. Coincident with the assimilative processes, a series of disintegrative processes is constantly taking place, whereby the living material is reduced, through a series of downward chemic changes, to 
simpler compounds, such as water, carbon dioxid, urea, etc. To all these downward changes the term dissimilation, or kataboiism, has been given. As a result, also, of these various changes, the protoplasm gives rise to the production of material of an entirely different character, such as globules of fat, granules of glycogen, mucigen, digestive ferments, etc. The sum total of all changes which go on in the cell, both assimilative and dissimilative, are embraced under the general term nutrition, or metabolism. Every cell presents in its nutritive activities an epitome of the nutritive activities of the body as a whole.

Physiologic Properties of Protoplasm.-All living protoplasm possesses properties which serve to distinguish and characterize it-viz., irritability, conductivity, and motility.

Irritability, or the power of reacting in a definite manner to some form of external excitation, whether mechanical, chemic, or electric, is a fundamental property of all living protoplasm. The character and extent of the reaction will vary, and will depend both on the nature of the protoplasm and the character and strength of the stimulus. If the protoplasm be muscle, the response will be a contraction ; if it be gland, the response will be secretion; if it be nerve, the response will be a sensation or some other form of nerve activity.

Conductivity, or the power of transmitting molecular disturbances arising at one point to all portions of the irritable material, is also a characteristic feature of all protoplasm. This power, however, is best developed in that form of protoplasm found in nerves, which serves to transmit, with extreme rapidity, molecular disturbances arising at the periphery to the brain, as well as in the reverse direction. Muscle protoplasm also possesses the same power in a high degree.

Motility, or the power of executing apparently spontaneous movements, is exhibited by many forms of cell protoplasm. In addition to the molecular movements which take place in certain cells, other forms of movement are exhibited, more or less constantly, by many cells in the animal bodye. $g$., the waving of cilia, the ameboid movements and migrations of white blood-corpuscles, the activities of spermatozooids, the projections of pseudopodia, etc. These movements, arising without any recognizable cause, are frequently spoken of as spontaneous. Strictly speaking, however, all protoplasmic movement is the resultant of natural causes, the true nature of which is beyond the reach of present methods of investigation.

Reproduction.-Cells reproduce themselves in the higher animals in two ways-by direct division and by indirect division, or karyokinesis. In 
the former the nucleus becomes constricted, and divides without any special grouping of the nuclear elements. It is probable that this occurs only in disintegrating cells, and never in a physiologic multiplication. In division by karyokinesis (Fig. 3) there is a progressive rearranging and definite grouping of the nucleus, the result of which changes is the division of the centrosome, the chromatin, and the rest of the nucleus into two equal portions, which form the nuclei. Following the division of the nuclei, the protoplasm divides. The process may be divided into three phases:

Close Skein

(viewed from the side). Polar field.
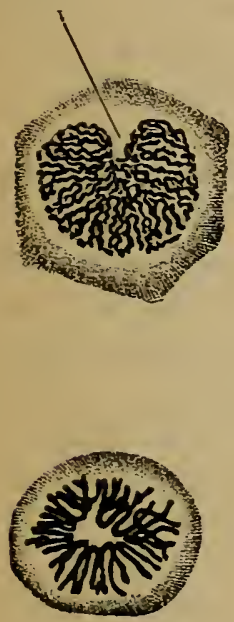

Mother Star (viewed from above).

\section{Loose Skein (viewed} from above $-i . e$. , from the pole).
Mother Stars (viewed from the side).
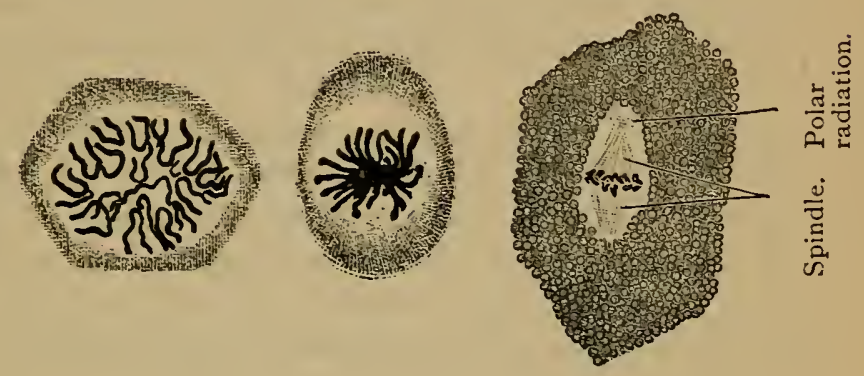

Fig. 3.-Karyokinetic Figures Observed in the Epithelium of the Oral Cavity of a Salamander.

The picture in the upper right-hand corner is from a section through a dividing egg of Siredon pisciformis. Neither the centrosomes nor the first stages of the development of the spindle can be seen by this magnification. $\times 560$.

I. Prophase.-The centrosoma, at first small and lying within the nucleus, increases in size and moves into the protoplasm, where it lies near the nucleus, surrounded by a clear zone, from which delicate threads radiate through an area known as the attraction sphere. The nucleus enlarges and becomes richer in chromatin. The lateral twigs of the chromatin cords are drawn in, while the main cords become much contorted. These 
cords have a general direction transverse to the long axis of the cell, and parallel to the plane of future cleavage. They are seen as V-shaped segments or loops, chromosomes, having their closed ends directed toward a common center, the polar field, while the other ends interdigitate on the opposite side of the nucleus-the anti-pole. The polar field corresponds to the area occupied by the centrosoma. This arrangement is known as the close skein; but as the process goes on, the chromosomes become thicker, shorter and less contorted, producing a much looser arrangement, known as the loose skein. During the formation of the loose skein, the centrosoma divides into two portions, which move apart to positions at the opposite ends of the long axis of the nucleus. At the same time delicate achromatin fibers make their appearance, arranged in the form of a double cone, the apices of which correspond in position to the centrosoma. This is known as the nuclear spindle. During the prophase the nuclear membrane and the nucleoli disappear.

2. The Metaphase. - The two centrosomata are at opposite ends of the long axis of the nucleus, each surrounded by an attraction sphere, now called the polar radiation. The chromosomes become yet shorter and thicker, and move toward the equator of the nucleus, where they lie with their closed ends toward the axis, presenting the appearance, when seen from the poles, of a star, - the so-called mother star, or monaster. While moving toward the equator of the nucleus, and often earlier, each chromosome undergoes longitudinal cleavage, the sister loops remaining together for a time. Upon the completion of the monaster, one loop of each pair passes to each pole of the nucleus, guided, and perhaps drawn by the threads of the nuclear spindle. The separation of the sister segments begins at their apices, and as the open ends are drawn apart they remain connected by delicate achromatin filaments drawn out from the chromosomes. This separation of the daughter chromosomes, and their movement toward the daughter centrosomata, is called metakinesis. As they approach their destination, we have the appearance of two stars in the nucleus-the daughter stars, or diasters.

3. Anaphase.- The daughter stars undergo, in reverse order, much the same changes that the mother star passed through. The chromosomes become much convoluted, and perhaps united to one another, the lateral twigs appear, and the chromatin resumes the appearance of the resting nucleus. The nuclear spindle, with most of the polar radiation, disappears, and the nucleoli and the nuclear membrane reappear, thus forming two complete daughter nuclei. Mean while the protoplasm becomes constricted midway between the young nuclei. This constriction gradually 
deepens until the original cell is divided, with the formation of two complete cells.

\section{HISTOLOGY OF THE EPITHELIAL AND CONNECTIVE TISSUES.}

\section{EPITHELIAL TISSUE.}

The epithelial tissue consists of one or more layers of cells resting on a homogeneous membrane, the other side of which is abundantly supplied with blood-vessels and nerves. The form of the epithelial cell varies in different situations, and may be flattened, cuboid, spheroid, or columnar. The form of the cell in all instances is related to some specific function. When arranged in layers or strata, the cells are cemented together by an intercellular substance-mucin.

The epithelial tissue forms a continuous covering for the surfaces of the body. The external investment (the skin) and the internal investment (the mucous membrane, which lines the entire alimentary canal and its associated body cavities) are both formed, in all situations, by the homogeneous basement membrane, covered with one or more layers of cells. All materials, therefore, whether nutritive, secretory, or excretory, must pass through epithelial cells before they can enter into the formation of the tissues or be eliminated from them. The nutrition of the epithelial tissue is maintained by the nutritive material derived from the blood diffusing itself into and through the basement membrane. Chemically, the epithelial cells of the epidermis-hair, nails, etc.-are composed of an albuminoid material (keratin), a small quantity of water, and inorganic salts. In other situations, especially on the mucous membranes, the cells consist largely of mucin, in association with other albuminoids. The consistency of epithelium varies in accordance with external influences, such as the presence or absence of moisture, pressure, friction, etc. This is well seen in the skin of the palms of the hands and the soles of the feet-situations where it acquires its greatest density. In the alimentary canal, in the lungs, and in other cavities, where the reverse conditions prevail, the epithelium is extremely soft. Epithelial tissues also possess varying degrees of cohesion and elasticityphysical properties which enable them to resist considerable pressure and distention without having their physiologic integrity destroyed. Inasmuch as these tissues are poor conductors of heat, they assist in preventing too rapid radiation of heat from the body, and coöperate with other mechanisms in maintaining the normal temperature. The physiologic activity of all epithelial tissue depends on a due supply of nutritive material derived from 
the blood, which not only maintains its own nutrition, but affords those materials out of which are formed the secretions of the glands, whether of the skin or mucous membrane.

Functions of Epithelial Tissue.-In succeeding chapters the form, chemic composition, and functions of epithelial cells will be considered in connection with the functions of the organs of which they constitute a part. In this connection it may be stated in a general way that the functions of the epithelial tissues are :

I. To serve on the surface of the body as a protective covering to the underlying structures 'which collectively form the true skin, thus protecting them from the injurious influences of moisture, air, dust, microörganisms, etc., which would otherwise impair their vitality. Wherever continuous pressure is applied to the skin, as on the palms of the hands and soles of the feet, the epithelium increases in thickness and density, and thus prevents undue pressure on the nerves of the true skin. The density of the epidermis enables it to resist, within limits, the injurious influences of acids, alkalies, and poisons.

2. To promote absorption. Inasmuch as the skin and mucous membranes cover the surfaces of the body, it is obvious that all nutritive material entering the body must first traverse the epithelial tissue. Owing to their density, however, the epithelial cells covering the skin play but a feeble rôle as absorbing agents in man and the higher animals. The epithelium of the mucous membrane of the alimentary canal, particularly that of the small intestine, is especially adapted, from its situation, consistency, and properties, to play the chief rôle in the absorption of new materials into the blood. The epithelium lining the air-vesicles of the lungs is engaged in promoting the absorption of oxygen and the exhalation of carbon dioxid.

3. To form secretions and excretions. Each secretory gland connected with the surfaces of the body is lined by epithelial cells, which are actively concerned in the formation of the secretion peculiar to the gland. Each excretory organ is similarly provided with epithelial cells, which are engaged either in the production of the constituents of the excretion or in their removal from the blood.

\section{THE CONNECTIVE TISSUES.}

The connective tissues, in their collective capacity, constitute a framework which pervades the body in all directions, and, as the name implies, serve as a bond of connection between the individual parts, at the same 
time affording a basis of support for the muscle, nerve, and gland tissues. The connective-tissue group includes a number of varieties, among which may be mentioned the areolar, adipose, retiform, white fibrous, yellow elastic, cartilaginous and osseous. Notwithstanding their apparent diversity, they possess many points of similarity. They have a common origin, developing from the same embryonic material; they have much the same structure, passing imperceptibly into one another, and perform practically the same functions.

Areolar Tissue.-This variety is found widely distributed throughout the body. It serves to unite the skin and mucous membrane to the structures on which they rest ; to form sheaths for the support of blood-vessels, nerves, and lymphatics; to unite into compact masses the muscular tissue of the body, etc. Examined with the naked eye, it presents the appearance of being composed of bundles of fine fibers interlacing in every direction. In the embryonic state the elements of this form of connective tissue are united by a ground substance, gelatinous in character. In the adult state this substance shrinks and largely disappears, leaving intercommunicating spaces of varying size and shape, from which the tissue takes its name. When subjected to the action of various reagents, and examined microscopically, the bundles can be shown to consist of extremely delicate, colorless, transparent, wavy fibers, which are cemented together by a ground substance composed largely of mucin. Other fibers are also observed, which are distinguished by a straight course, a sharp, well-defined outline, a tendency to branch and unite with adjoining fibers, and to curl up at their extremities when torn. From their color and elasticity they are known as yellow elastic fibers. Distributed throughout the meshes of the areolar tissue are found flattened, irregularly branched, or stellate corpuscles, connective-tissue corpuscles, plasma cells, and granule cells.

Adipose Tissue.-This tissue, which exists very generally throughout the body, though found most abundantly beneath the skin, around the kidneys, and in the bones, is practically but a modification of areolar tissue. In these situations it presents itself in small masses or lobules of varying size and shape, surrounded and penetrated by the fibers of connective tissue. Microscopic examination shows that these masses consist of small vesicles or cells, round, oval, or polyhedral in shape, depending somewhat on pressure. Each vesicle consists of a thin, colorless, protoplasmic membrane, thickened at one point, in which a nucleus can usually be detected. This membrane incloses a globule of fat, which during life is in the liquid state. It is composed of olein, stearin, and palmitin. The origin of the 
fat is to be referred to a retrograde change in the protoplasmic material of the connective-tissue cells. When this protoplasm becomes rich in carbon and hydrogen, it is speedily converted into fat, which makes its appearance in the form of minute drops in different portions of the cell. As the drops accumulate, at the expense of the cell protoplasm, they gradually coalesce, until there remains but a thin stratum of the protoplasm, which forms the wall of the vesicle. Adipose tissue may, therefore, be regarded as areolar tissue, in which, and at the expense of some of its elements, fat is stored for the future needs of the organism. A diminution of food, especially of fat and carbohydrates, is promptly followed by an absorption of fat by the blood-vessels and by its transference to the tissues, where it is either utilized for tissue construction or for oxidation purposes. In the situations in which adipose tissue is found it seems, by its chemic and physical properties, to assist in the prevention of a too rapid radiation of heat from the body, to give form and roundness, and to diminish angularities, etc.

Retiform and adenoid tissue are also modifications of areolar tissue. The meshes of the former contain but little ground substance, its place being taken by fluids; the meshes of the latter contain large numbers of lymph corpuscles.

Fibrous Tissue.-This variety of connective tissue is widely distributed throughout the body. It constitutes almost entirely the ligaments around the joints, the tendons of the muscles, the membranes covering organs such as the heart, liver, nervous system, bones, etc. All fibrous tissue, wherever found, can be resolved into elementary bundles, which on microscopic examination are seen to consist of delicate, wavy, transparent, homogeneous fibers, which pursue an independent course, neither branching nor uniting with adjoining fibers. A small amount of ground substance serves to hold them together. Fibrous tissue is tough and inextensible, and in consequence is admirably adapted to fulfil various mechanical functions in the body. It is, however, quite pliant, bending easily in all directions. When boiled, fibrous tissue yields gelatin, a derivative of collagen.

Elastic Tissue.-The fibers of elastic tissue are usually associated in varying proportions with the white fibrous tissue ; but in some structuresas the ligamentum nuchæ, the ligamenta subflava, the middle coat of the larger blood-vessels-the elastic fibers are almost the only elements present, and give to these structures a distinctly yellow appearance. The fibers throughout their course give off many branches, which unite with adjoining branches to form a more or less close network. As the name implies, 
these fibers are highly elastic, and are capable of being extended as much as sixty per cent. before breaking.

Cartilaginous Tissue.-This form of connective tissue differs from the preceding varieties chiefly in its density. As a rule, it is firm in consistency, though somewhat elastic. It is opaque, bluish-white in color, though in thin sections translucent. All cartilaginous tissues consist of connective-tissue cells embedded in a solid ground substance. According to the amount and texture of the ground substance, three principal varieties may be distinguished : I. Hyaline cartilage, in which the cells, relatively few in number, are embedded in an abundant quantity of ground substance. The body of the cells is in many instances distinctly marked off from the surrounding substance by concentric lines or fibers, which form a capsule for the cell. Repeated division of the cell substance takes place, until the whole capsule is completely occupied by daughter cells. The ground substance is pervaded by minute channels, which communicate on one hand with the spaces around the cells, and on the other with lymph-spaces in the connective tissue surrounding the cartilage. By means of these channels, nutritive fluid can permeate the entire structure. Hyaline cartilage is found on the ends of the long bones, where it enters into the formation of the joints; between the ribs and sternum, forming the costal cartilage, as well as in the nose and larynx.

2. White fibro-cartilage, the ground substance of which is pervaded by white fibers, arranged in bundles or layers, between which are scattered the usual encapsulated cells. White fibro-cartilage is tough, resistant, but flexible, and is found in joints where strength and fixedness are required. Hence it is present between the vertebræ, forming the intervertebral discs, between the condyle of the lower jaw and the glenoid fossa, in the knee-joint, around the margins of the joint cavities, etc. In these situations it assists in maintaining the apposition of the bones, in giving a certain degree of mobility to the joints, and in diminishing the effects of shock and pressure imparted to the bones.

3. Yellow fibro-cartilage, the ground substance of which is pervaded by opaque, yellow elastic fibers, which form, by the interlacing of their branches, a complicated network, in the meshes of which are to be found the usual corpuscles. As these fibers are elastic, they impart to the cartilage a very considerable degree of elasticity. Yellow fibro-cartilage is well adapted, therefore, for entering into the formation of the external ear, epiglottis, Eustachian tube, etc.-structures which require for their functional activity a certain degree of flexibility and elasticity. 
Osseous Tissue.-Osseous tissue, as distinguished from bone, is a member of the connective-tissue group, the ground substance of which is permeated with insoluble lime salts, of which the phosphate and carbonate are the most abundant. Immersed in dilute solutions of hydrochloric acid, they can be converted into soluble salts and dissolved out. The osseous matrix left behind is soft and pliable. When boiled, it yields gelatin.

A thin, transverse section of a decalcified bone, when examined microscopically, reveals a number of small, round or oval openings, which represent transverse sections of canals which run through the bone, for the most part in a longitudinal direction, though frequently anastomosing with one another. These so-called Haversian canals in the living state contain blood-vessels and lymphatics.

Around each Haversian canal is a series of concentric laminæ, composed of white fibers. Between every two laminæ are found small cavities (lacunæ), from which radiate in all directions small canals (canaliculi), which communicate freely with one another. The Haversian canals, with their associated lacunæ and canaliculi, form a system of intercommunicating passages, through which lymph circulates destined for the nourishment of bone. Each lacuna contains the bone corpuscle, which bears a close resemblance to the usual branched connective-tissue corpuscle, and whose function appears to be the maintenance of the nutrition of the bone.

The surface of every bone in the recent state is invested with a fibrous membrane, the periosteum, except where it is covered with cartilage. The inner surface of this membrane is loose in texture, and supports a fine plexus of capillary blood-vessels and numerous protoplasmic cells-the osteoblasts. As this layer is directly concerned in the formation of bone, it is spoken of as the osteogenetic layer.

A section of a bone shows that it is composed of two kinds of tissuecompact and cancellated. The compact is dense, resembling ivory, and is found on the outer portion of the bone; the cancellated is spongy, and appears to be made up of thin, bony plates, which intersect one another in all directions, and is found in greatest abundance in the interior of the bones. The shaft of a long bone is hollow. This central cavity, which extends from one end of the bone to the other, as well as the interstices of the cancellated tissue, is filled in the living state with marrow. The marrow or medulla is composed of a connective-tissue framework supporting bloodvessels. In its meshes are to be found characteristic bone cells or osteoblasts, the function of which is supposed to be the formation of bone. In 
the long bones the marrow is yellow, from the presence in the connectivetissue corpuscle of fat globules, which arise through the transformation of the cell protoplasm. In the cancellated tissue, near the extremities of the long bones, this fatty transformation does not take place to the same extent, and the marrow appears red. The cells of the red marrow are believed to give birth indirectly to the red blood-corpuscles.

Physical and Physiologic Properties of Connective Tissues.Among the physical properties may be mentioned consistency, cohesion, and elasticity. Their consistency varies from the semiliquid to the solid state, and depends on the quantity of water which enters into their composition. Their cohesion, except in the softer varieties, is very considerable, and offers great resistance to traction, pressure, torsion, etc. In all the movements of the body, in the contraction of muscles, in the performance of work, the consistence and cohesion of these tissues play most important roles. Wherever the various forms of connective tissue are found, their chemic composition and structure are in relation to their functions. If traction be the preponderating force, the structure becomes fibrous, as in ligaments and tendons, and the cohesion greatest in the longitudinal direction. If pressure be exerted in all directions, as upon membranes, the fibers interlace and offer a uniform resistance. When pressure is exerted in a definite direction, as on the extremities of the long bones, the tissue becomes expanded and cancellated. The lamellæ of the cancellated tissue arrange themselves in curves which correspond to the direction of the greatest pressure or traction. Extensibility is not a characteristic feature, except in those forms containing an abundance of yellow elastic fibers. The elasticity is an essential factor in many physiologic actions. It not only opposes and limits forces of traction, pressure, torsion, etc., but on their cessation returns the tissues or organs to their original condition. Elasticity thus assists in maintaining the natural form and position of the organs by counterbalancing and opposing temporarily acting forces.

The Skeleton.-The connective tissues in their entirety constitute a framework which presents itself under two aspects: (I) As a solid, bony skeleton, situated in the trunk and limbs, affording attachment for muscles and viscera; (2) as a fine, fibrons skeleton, found everywhere throughout the body, connecting the various viscera and affording support for the epithelial, muscle, and nerve tissues. 


\section{THE PHYSIOLOGY OF THE SKELETON.}

The animal body is characterized by the power of executing a great variety of movements, all of which have reference to a change of relation of one part of the body to another, or to a change of position of the individual in space, as in the various acts of locomotion. If in the execution of these movements the different parts are applied or directed to the overcoming of opposing forces in the environment, the animal is said to be doing work. In the conception of the animal body as a machine for the accomplishment of work the skeleton, the muscle and nerve tissues constitute the three primary mechanisms, all of which bear certain definite relations one to another.

The Skeleton is the passive framework, the axial portion of which (the vertebral column, head, ribs, and sternum) impart more or less fixity and rigidity, while the appendicular portions (the bones of the arms and legs) impart extreme mobility. The bones of the arms and legs more especially may be looked upon as constituting a system of levers, the fulcra of which, the points of rest around which they move, lie in the joints.

That a lever may be effective as an instrument for the accomplishment of work, it must not only be capable of moving around its fulcrum, but it must at the same time be acted on by two opposing forces, one passive, the other active. In the movement of the bony levers of the animal body, the passive forces are largely those connected with the environment, e. g., gravity, cohesion, friction, elasticity, etc. The active forces by which these latter are opposed and overcome through the intermediation of the bony levers are found in the muscles attached to them. For the execution of all these movements, it is essential that the relation of the various portions of the bony skeleton to one another shall be such as to permit of movement while yet retaining close apposition. This is accomplished by the mechanical conditions which have been evolved at the points of union of bones, and which are technically known as articulations or joints.

A consideration of the body movements involves an account of (I) the static conditions, or those states of equilibrium in which the body is at rest-e. $g$. , standing, sitting; (2) the dynamic conditions, or those states of activity characterized by movement-e. $g$., walking, running, etc. In this connection, however, only those physical and physiologic peculiarities of the skeleton, especially in its relation to joints, will be referred to which underlie and determine both the static and dynamic states of the body. 
Structure of Joints.- The structures entering into the formation of joints are :

I. Bones, the articulating surfaces of which are often more or less expanded, especially in the case of long bones, and at the same time variously modified and adapted to one another in accordance with the character and extent of the movements which there take place.

2. Hyaline cartilage, which is closely applied to the articulating end of each bone. The smoothness of this form of cartilage facilitates the movements of the opposing surfaces, while its elasticity diminishes the force of shocks and jars imparted to the bones during various muscular acts. In a number of joints, plates or discs of white fibro-cartilage are inserted between the surfaces of the bones.

3. A synovial membrane, which is attached to the edge of the hyaline cartilage, entirely inclosing the cavity of the joint. This membrane is composed largely of connective tissue, the inner surface of which is lined by endothelial cells, which secrete a clear, colorless, viscid fluid-the synovia. This fluid not only fills up the joint-cavity, but, flowing over the articulating surfaces, diminishes or prevents friction.

4. Ligaments, - tough, inelastic bands, composed of white fibrous tissue, which pass from bone to bone in various directions on the different aspects of the joint. As white fibrous tissue is inextensible but pliant, ligaments assist in keeping the bones in apposition, and prevent displacement while yet permitting of free and easy movements.

Classification of Joints.-All joints may be divided, according to the extent and kind of movements permitted by them, into (I) diarthroses; (2) amphiarthroses; (3) synarthroses.

I. Diarthroses. - In this division of the joints are included all those which permit of free movement. In the majority of instances the articulating surfaces are mutually adapted to each other. If the articulating surface of one bone is convex, the opposing but corresponding surface is concave. Each surface, therefore, represents a section of a sphere or a cylinder, which latter arises by rotation of a line around an axis in space. According to the number of axes around which the movements take place all diarthrodial joints may be divided into :

I. Uniaxial Joints.-In this group the convex articulating surface is a segment of a cylinder or cone, to which the opposing surface more or less completely corresponds. In such a joint the single axis of rotation, though, practically is not exactly at right angles to the long axis of the bone, and hence the movements-flexion and extension-which take place are not confined to one plane. Joints of this character-e. $g$., the elbow, 
knee, ankle, the phalangeal joints of the fingers and toes-are, therefore, termed ginglymi, or hinge-joints. Owing to the obliquity of their articulating surfaces, the elbow and ankle are cochleoid or screw-ginglymi. Inasmuch as the axes of these joints on the opposite sides of the body are not coincident, the right elbow and left ankle are right-handed screws; the left elbow and right ankle, left-handed screws. In the knee-joint the form and arrangement of the articulating surfaces are such as to produce that modification of a simple hinge known as a spiral hinge, or helicoid. As the articulating surfaces of the condyles of the femur increase in convexity from before backward, and as the inner condyle is longer than the outer, and, therefore, represents a spiral surface, the line of translation or the movement of the leg is also a spiral movement. During flexion of the leg there is a simultaneous inward rotation around a vertical axis passing through the outer condyle of the femur; during extension a reverse movement takes place. Moreover, the slightly concave articulating surfaces of the tibia do not revolve around a single fixed transverse axis, as in the elbow-joint, for during flexion they slide backward, during extension forward, around a shifting axis, which varies in position with the point of contact.

In some few instances the long axis of the articulating surface is parallel rather than transverse to the long axis, and as the movement then takes place around a more or less conic surface, the joint is termed a trochoid or pulley-e. g., the odonto-atlantal and the radio-ulnar. In the former the collar formed by the atlas and its transverse ligament rotates around the vertical odontoid process of the axis. In the latter the head of the radius revolves around its own long axis upon the ulna, giving rise to the movements of pronation and supination of the hand. The axis around which these two movements take place is continued through the head of the radius to the styloid process of the ulna.

2. Biaxial Joints. - In this group the articulating surfaces are unequally curved, though intersecting each other. When the surfaces lie in the same direction, the joint is termed an ovoid joint-e. $g$., the radio-carpal and the atlanto-occipital. As the axes of these surfaces are vertical to each other, the movements permitted by the former joint are flexion, extension, adduction, and abduction, combined with a slight amount of circumduction; the latter joint permits of flexion and extension of the head, with inclination to either side. When the surfaces do not take the same direction, the joint, from its resemblance to the surfaces of a saddle, is termed a saddle-joint-e. g., the trapezio-metacarpal. The movements permitted by this joint are also flexion, extension, adduction, abduction, and circumduction. 
3. Polyaxial Joints. - In this group the convex articulating surface is a segment of a sphere, which is received by a socket formed by the opposing articulating surface. In such a joint, termed an cnarthrodial or ball-and-socket joint, - e. $g$., the shoulder-joint, hip-joint, - the distal bone revolves around an indefinite number of axes, all of which intersect one another at the center of rotation. For simplicity, however, the movement may be described as taking place around axes in the three ordinal planes-viz., a transverse, a sagittal, and a vertical axis. The movements around the transverse axis are termed flexion and extension; around the sagittal axis, adduction and abduction; around the vertical axis, rotation. When the bone revolves around the surface of an imaginary cone, the apex of which is the center of rotation and the base the curve described by the hand, the movement is termed circumduction.

2. Amphiarthroses. - In this division are included all those joints which permit of but slight movement-e. $g$., the intervertebral, the interpubic, and the sacro-iliac joints. The surfaces of the opposing bones are united and held in position largely by the intervention of a firm, elastic disc of fibro-cartilage. Each joint is also strengthened by ligaments.

3. Synarthroses. - In this division are included all those joints in which the opposing surfaces of the bones are immovably united, and hence do not permit of any movement-e.g., the joints between the bones of the skull.

The Vertebral Column.-In all static and dynamic states of the body the vertebral column plays a most essential rôle. Situated in the middle of the back of the trunk, it forms the foundation of the entire skeleton. It is composed of a series of superimposed bones, termed vertebræ, which increase in size from above downward as far as the brim of the pelvic cavity. Superiorly, it supports the skull; laterally, it affords attachment for the ribs, which in turn support the weight of the upper extremities; below, it rests upon the pelvic bones, which transmit the weight of the body to the inferior extremities. The bodies of the vertebre are united one to another by tough elastic discs of fibro-cartilage, which, collectively, constitute about one quarter of the length of the vertebral column. The vertebræ are held together by ligaments situated on the anterior and posterior surfaces of their bodies, and by short, elastic ligaments between the neural arches and processes. These structures combine to render the vertebral column elastic and flexible, and enable it to resist and diminish the force of shocks communicated to it. 
The amphiarthrodial character of the intervertebral joints endows the entire column with certain forms of movement which are necessary to the performance of many body activities. While the range of movement between any two vertebræ is slight, the sum total of movement of the entire series of vertebræ is considerable. In different regions of the column the character, as well as the range of movement, varies in accordance with the form of the vertebræ and the inclination of their articular processes. In the cervical and lumbar regions extension and flexion are freely permitted, though the former is greater in the cervical, the latter in the lumbar region, especially between the fourth and fifth vertebræ. Lateral flexion takes place in all portions of the column, but is particularly marked in the cervical region. A rotatory movement of the column as a whole takes place through an angle of about twenty-eight degrees. This is most evident in the lower cervical and dorsal regions.

The skeleton may, therefore, be regarded as a highly developed framework, which determines not only the form of the body, and affords support and protection to the various softer organs and tissues, but also, through the mobility of its joints, permits of a great variety of complicated movements.

\section{GENERAL PHYSIOLOGY OF MUSCLE TISSUE.}

The muscle tissue, which closely invests the bones of the body, and which is familiar to all as the flesh of animals, is the immediate cause of the active movements of the body. This tissue is grouped in masses of varying size and shape, which are technically known as muscles. The majority of the muscles of the body are connected with the bones of the skeleton in such a manner that, by an alteration in their form, they can change not only the position of the bones with reference to one another, but can also change the individual's relation to surrounding objects. They are, therefore, the active organs of both motion and locomotion, in contradistinction to the bones and joints, which are but passive agents in the performance of the corresponding movements. In addition to the muscle masses which are attached to the skeleton, there are also other collections of muscle tissue surrounding cavities such as the stomach, intestine, bloodvessels, etc., which impart to their walls motility, and so influence the passage of material through them.

Muscles produce movement of the structures to which they are attached by the property with which they are endowed of changing their shape, 
shortening or contracting under the influence of a stimulus transmitted to them from the nervous system. Muscles are therefore divided into:

1. Voluntary muscles, comprising those whose activity is called forth by stimuli of the nerves as the result of an act or effort of volition.

2. Involuntary muscles, comprising those whose activity is entirely independent of the volition.

The voluntary muscles are also known from their attachment to the skeleton as skelctal, and from their microscopic appearance. as striped muscles. The involuntary muscles, from their relation to the viscera of the body, are known also as visceral, and from their microscopic appearance as plain or smooth muscles.

General Structure of Muscles.-All skeletal muscles consist of a central fleshy portion, the body or belly, which is provided at either extremity with a tendon in the form of a cord or membrane by which it is attached to the bones. The body is the contractile region, the source of activity; the tendon is a passive region, and merely transmits the activity to the bones.

A skeletal muscle is a complex organ consisting of muscular fibers, connective tissue, blood-vessels, and lymphatics. The general body of the muscle is surrounded by a dense layer of connective tissue, the epimysium, which blends with and partly forms the tendon; from its inner surface septa of connective tissue pass inward and group the muscle-fibers into larger and smaller bundles, termed fasciculi. The fasciculi, invested by this special sheath, the perimysizım, are irregular in shape, and vary considerably in size. The fibers of the fasciculi are separated from one another and supported by a delicate connective tissue, the endomysizm. The connective tissue thus surrounding and penetrating the muscle binds its fibers into a distinct organ, and affords support to blood-vessels, nerves, and lymphatics. The muscle-fibers are arranged parallel to one another, and their direction is that of the long axis of the muscle. In length they vary from thirty to forty millimeters, and in diameter from twenty to thirty micromillimeters.

The vascular supply to the muscles is very great, and the disposition of the capillary vessels, with reference to muscle-fiber, is very characteristic. The arterial vessels, after entering the muscle, are supported by the perinysium; in this situation they give off short, transverse branches, which immediately break up into a capillary network of rectangular shape, within which the muscle-fibers are contained. The muscle-fiber in intimate relation with the capillary is bathed with lymph derived from it. Its 
contractile substance, however, is separated from the lymph by its own investing membrane, through which all interchange of nutritive and waste materials must take place. Lymphatics are present in muscle, but are confined to the connective tissue, in the spaces of which they have their origin.

The nerves which carry the stimuli to a muscle enter near its geometric center. Many of the fibers pass directly to the muscle-fibers with which they are connected; others are distributed to blood-vessels. Every muscle-fiber is supplied with a special nerve-fiber, except in those instances where the nerve trunks entering a muscle do not contain so many fibers as the muscle. In such cases the nerve-fibers divide, until the number of branches equals the number of muscle-fibers. The individual musclefiber is penetrated near its center by the nerve, the ends being practically free from nerve influence. The stimulus that comes to the muscle-fiber acts primarily upon its center, and then travels in both directions to the ends.

Histology of the Skeletal Muscle-fiber.-A muscle-fiber consists of a transparent elastic membrane, the sarcolemma, in which is contained the true muscle element. Examined microscopically, the fiber presents a series of alternate dim and bright bands, giving to it a striated appearance.

When the bright band is examined with high magnifying powers, a fine, dark line is seen crossing it transversely. It was supposed by Krause to be the optic expression of a membrane which divides the cavity of the sarcolemma into a series of compartments, each of which contains a dim band of sarcous or muscle substance, bounded at either extremity with the half of a bright band. This membrane has since been resolved into a row of granules.

'The muscle-fiber also exhibits a longitudinal striation, indicating that it is composed of fibrillæe, placed side by side and embedded in some interfibrillar substance, to which the name sarcoplasm has been given. The fibrillæ, which are arranged longitudinally to the long axis of the fiber, are grouped by the intervening material into bundles of varying size, the muscle columns. The fibrillæ which extend throughout the length of the fiber are not of uniform thickness, but present at regular intervals wellmarked constrictions.

In the region of the dim band the fibrilla presents itself in the form of a homogeneous prismatic rod, termed sarcostyle, separated from neighboring rods by a slight amount of sarcoplasm. Between two successive rods is found a dark granule, united by a thin band of similar ma erial to the ends of the rods. The transverse row of granules corresponds to Krause's membrane. 
In the region of the granules there is a diminution of the sarcous substance, but an increase in the amount of sarcoplasm, and as the latter is more transparent than the former, the fiber presents at this point a conspicuous bright band. Rollet considers the sarcostyles to be preëxistent, not the result of post-mortem or chemic changes, and the seat of the contractile elements. The sarcoplasm is a passive material similar in its properties to protoplasm.

Brücke has shown that when the muscle-fiber is examined under crossed Nichol prisms the dim band appears bright and the bright band appears dim againșt a dark background, indicating that the former is doubly refractile, or anisotropic, the latter singly refractile, or isotropic. The fiber, therefore, appears to be composed of alternate discs of anisotropic and isotropic substance.

Structure of Non-striated Muscle-fiber.-As the name implies, the involuntary fiber is non-striated, being apparently uniform and homogeneous in appearance. When isolated, the fiber presents itself in the form of an elongated fusiform cell, varying from $\frac{1}{10}$ to $\frac{1}{60}$ of an inch in length. In some animals the fiber exhibits a longitudinal striation, as if it were composed of fibers. The cell is surrounded by a thin, elastic membrane, and contains a distinct oval nucleus. The fibers are usually arranged in bundles and lamellæ, and held together by a cement substance and connective tissue. This non-striated muscle tissue is found in the muscularis mucosæ of the alimentary canal as well as in the muscular walls of the stomach and intestines, in the posterior part of the trachea, in the bronchial tubes, in the walls of the blood-vessels, and in many other situations.

Chemic Composition of Muscle.-The chemic composition of muscle is imperfectly understood, owing to the fact that some of its constituents undergo a spontaneous coagulation after death, and that the chemic methods employed also tend to alter its normal composition. When fresh muscle is freed from fat and connective tissue, frozen, rubbed up in a mortar, and expressed through linen, a slightly yellow, syrupy, alkaline, or neutral fluid is obtained, known as muscle plasma. This fluid at normal temperature coagulates spontaneously, and resembles in many respects the coagulation of blood plasma. The coagulum subsequently contracts and squeezes out an acid muscle serum. The coagulated mass is termed myosin. This proteid belongs to the class of globulins. Inasmuch as it is not present in living muscle, and makes its appearance only in the as yet living muscle plasma, it is probable that it is derived from some preëxisting sub- 
stance, which is supposed to be myosinogen. Myosin is digested by pepsin and trypsin. According to Halliburton, muscle plasma contains the following proteid bodies: Myosinogen, paramyosinogen, albumin, myoalbumose, all of which differ in chemic composition and respond to various chemic and physical reagents.

Ferment bodies, such as pepsin and diastase; non-nitrogenized bodies, such as glycogen, lactic and sarcolactic acids, fatty bodies, and inosite; nitrogenized extractives-e. g., urea, uric acid, kreatinin, as well as inorganic salts, have been obtained from the muscle serum.

Metabolism in Muscles.--The chemic changes which underlie the transformation of energy in living muscles are very active and complex.

As shown by an analysis of the blood flowing to and from the resting muscle, it has, while passing through the capillaries, lost oxygen and gained carbon dioxid. The amount of oxygen absorbed by the muscle (nine per cent.) is greater than the amount of $\mathrm{CO}_{2}$ given off ( 6.7 per cent.). There is no parallelism between these two processes, as $\mathrm{CO}_{2}$ will be given off in the absence of oxygen, or in an atmosphere of nitrogen.

In the active or contracting muscle both the absorption of oxygen and the production of $\mathrm{CO}_{2}$ are largely increased, but the ratio existing between them differs considerably from that of the resting muscle, for the quantity of oxygen absorbed amounts to $\mathrm{I} 1.26$ per cent., the quantity of $\mathrm{CO}_{2}$ to 10.8 per cent. (Ludwig). Moreover, in a tetanized muscle the quantity of $\mathrm{CO}_{2}$ given off may be largely in excess of the oxygen absorbed. From these facts it is evident that the energy of the contraction does not depend upon the direct oxidation of certain substances, but upon the decomposition of some unstable compound of high potential energy, rich in carbon and oxygen. When the muscle is active, its tissue changes from a neutral to an acid reaction, from the development of sarcolactic and possibly phosphoric acids. The amount of glycogen present in muscle ( 0.43 per cent.) diminishes, but muscles wanting in glycogen, nevertheless, retain their power of contraction. Water is absorbed. The amount of urea is not materially increased by muscular activity, unless it is excessive and prolonged, and then only in the absence of a sufficient quantity of nonnitrogenized material. Coincident with muscle contraction, the bloodvessels become widely dilated, leading to a large increase in the blood-supply and a rapid removal of products of decomposition.

Rigor Mortis.-A short time after death the muscles pass into a condition of extreme rigidity or contraction, which lasts from one to five days. In this state they offer great resistance to extension, their tonicity disap- 
pears, their cohesion diminishes, their irritability ceases. The time of the appearance of this post-mortem or cadaveric rigidity varies from a quarter of an hour to seven hours. Its onset and duration are influenced by the condition of the muscular irritability at the time of death. When the irritability is impaired from any cause, such as disease or defective bloodsupply, the rigidity appears promptly, but is of short duration. After death from acute diseases, it is apt to be delayed, but to continue for a longer period.

The rigidity appears first in the muscles of the lower jaw and neck; next in the muscles of the abdomen and upper extremities; finally in the trunk and lower extremities. It disappears in practically the same order.

Chenic changes of a marked character accompany this rigidity. The muscle becomes acid in reaction from the development of sarcolactic acid; it gives off a large quantity of carbonic acid, and is shortened and diminished in volume.

The immediate cause of the rigidity appears to be a coagulation of the myosinogen within the sarcolemma, with the subsequent formation of myosin and muscle serum. In the early stages of coagulation restitution is possible by the circulation of arterial blood through the vessels. The final disappearance of this contraction is due to the action of acids dissolving the myosin, and possibly to putrefactive changes.

Source of Muscular Energy.-According to most experimenters, it is certain that normal muscle activity is not dependent on the metabolism of nitrogenous materials, inasmuch as its chief end product, urea, is not increased. The marked production of $\mathrm{CO}_{2}$ points to the combustion of some non-nitrogenous matter, - e. g., glycogen,- - especially as this substance disappears during muscular activity. Muscles wanting in glycogen are, nevertheless, capable of contracting for some time. Moreover, there is no proof of the direct combustion of glycogen or any other carbohydrate. It has been suggested by Hermann that the energy of a muscular contraction may be due to the splitting and subsequent re-formation of a complex body belonging neither to the carbohydrates nor to the fats, but to the albumins. To this body the term inogen has been applied. This complex molecule, the product of the metabolic activity of the muscle cell, in undergoing decomposition would yield $\mathrm{CO}_{2}$, sarcolactic acid, and a proteid residue resembling myosin. With the cessation of the contraction, the muscle protoplasm recombines the proteid residue with oxygen, carbohydrates, and fats, and again forms inogen.

The phenomena of rigor mortis support such a view. At the moment of this contraction the muscle gives off $\mathrm{CO}_{2}$ in large amaunts, the muscle becomes acid, and myosin is formed, There is thus a close analogy 
between the two processes; in other words, a contraction is a partial death of the muscle. As to what becomes of the myosin formed during a contraction, nothing is known. It may be used in the formation of new inogen.

The Physical Properties of Muscle Tissue.-The consistency c' muscle tissue varies considerably, according to the different states of the muscle. In a state of tension it is hard and resistant; when free from tension, it is soft and fluctuating, whether the muscle is contracting or resting. Tension alone produces hardness. The cohesion of muscle tissue is less than that of connective tissue, and is broken more readily. Cohesion resists traction and pressure, and lasts as long as irritability remains.

The elasticity of a muscle, though not great is almost perfect. After being extended by a weight, it returns to its natural form. . The limit of elasticity, however, is soon passed. A weight of 50 or Ioo grams will overcome the elasticity so that it will not return to its natural length. In inorganic bodies the extension is directly proportional to the extending weight, and the line of extension is straight. With muscles, the extension is not proportional to the weight. While at first it is marked, the elongation diminishes as the weight increases by equal increments, so that the line of extension becomes a curve. In other words, the elasticity of a passive muscle augments with increased extension. On the contrary, the elasticity of an active is less than that of a passive muscle, for it is elongated more by the same weight, as shown by experiment.

Tonicity is a property of all muscles in the body, in consequence of being normally stretched to a slight extent beyond their natural length. This may be due to the action of antagonistic muscles, or to the elasticity of the parts of the skeleton to which they are attached. This is shown by the shortening of the muscle which takes place when it is divided. Muscular tonus plays an important rôle in muscular contraction. Being always on the stretch, the muscle loses no time in acquiring that degree of tension necessary to its immediate action on the bones. Again, the working power of a muscle is increased by the presence of some resistance to the act of contraction. According to Marey, the amount of work is considerably increased when the muscular energy is transmitted by an elastic body to the mass to be moved, while at the same time, the shock of the contraction is lessened. The position of a passive limb is the resultant also of the elastic tension of antagonistic groups of muscles.

Muscle excitability or contractility are terms employed to denote that property of muscle tissue in virtue of which it contracts or shortens in response to various excitants or stimuli. Though usually associated with 
the activity of the nervous system, it is, nevertheless, an independent endowment, and persists after all nervous connections are destroyed. If the nerve terminals be destroyed, as they can be by the introduction of curara into the system, the muscles become completely relaxed and quiescent. The strongest stimuli applied to the nerves fail to produce a contraction. Various external stimuli applied directly to the muscle substance produce at once the characteristic contraction. The excitability of muscle is therefore an inherent property, dependent on its nutrition, and persisting as long as it is supplied with proper nutritive materials and surrounded by those external conditions which maintain its chemic or physical integrity.

Muscle Contractions.-All muscle contractions occurring in the body under normal physiologic conditions are either voluntary, caused by a volitional effort and the transmission of a nerve impulse from the brain through the spinal cord and nerves to the muscles, or reflex, caused by a peripheral stimulation and the transmission of a nerve impulse to the spinal cord, to be reflected outward through the same nerves to the muscles. In either case the resulting contraction is essentially the same. The normal or physiologic stimulues which provokes the muscular contraction is a nerve impulse the nature of which is unknown, but is perhaps allied to a molecular disturbance. After removal from the body, muscles remain in a state of rest, inasmuch as they possess no spontaneity of action. Though consisting of a highly irritable tissue, they can not pass from the passive to the active state except upon the application of some form of stimulation.

The stimuli which are capable of calling forth a contraction may be divided into-

I. Mechanical.

2. Chemic.

3. Physical.

4. Electric.

Every mechanical stimulus of a muscle,-e. g., pick, cut, or tap,-providing it has sufficient intensity, and is repeated with sufficient rapidity, will cause not only a single contraction, but a series of contractions.

All chemic agents which impair the chemic composition of the muscle with sufficient rapidity-e.g., hydrochloric acid, acetic and oxalic acids, distilled water injected into the vessels, etc.-act as stimuli, and produce single and multiple contractions. Physical agents, as heat and electricity, also act as stimuli. A muscle heated rapidly to $30^{\circ} \mathrm{C}$. contracts vigorously, and reaches its maximum at $45^{\circ} \mathrm{C}$. Of all forms of stimuli, the 
electric is the most generally used. Two forms are used-the induced current and the make-and-break of a constant current.

Changes in a Muscle During Contraction.-When a muscle is stimulated, either indirectly through the nerve or directly by any external agent, it undergoes a series of changes, which relate to its form, volume, optic, physical, chemic, and electric properties. These changes, in their totality, constitute the muscular contraction.

I. Form.-The most obvious change is that of form. The fibers become shorter in their longitudinal and wider in their transverse diameters, and the muscle as a whole becomes shorter and thicker. The degree of shortening may amount to thirty per cent. of the original length.

2. Volume.-The increase in transverse diameter does not fully compensate for the diminution in length, for there is at the moment of contraction a slight shrinkage in volume, which has been attributed to a compression of air in its interstices.

3. Optic Changes. - If a muscle-fiber be examined microscopically during its contraction, it will be observed that when the contraction wave begins, both bright and dim bands diminish in height and become broader, though this change is more noticeable in the region of the bright band. This Englemann attributes to a passage of fluid material from the bright into the dim band. At the time of relaxation there is a return of this material, and the fiber assumes its original shape and volume. As the contraction wave reaches its maximum, the optic properties of both the isotropic and anisotropic bands change. The former, which was originally clear, now becomes darker and less transparent, until at the crest of the wave it assumes the appearance of a distinct dark band. The latter, the anisotropic, which was originally dim, now becomes, in comparison, clear and light. This change in optic appearance is due to an increase in refrangibility of the isotropic and a decrease in the anisotropic bands coincident with the passage of fluid from the former into the latter. There is at the height of the contraction a complete reversal in the positions of the striations. At a certain stage between the beginning and the crest of the wave there is an intermediate point, at which the strix almost entirely disappear, giving to the fiber an appearance of homogeneity. There is, however, no change in refractive power, as shown by the polarizing apparatus. After the contraction wave has reached the stage of greatest intensity, there is a reversal of the foregoing phenomena, and the fiber returns to its original condition, which is one of relaxation. 
Physical Changes. - The extensibility of muscle is increased during the contraction, the same weight elongating the fibers to a greater extent than during rest. The elasticity, or its power of returning to its original form is correspondingly diminished.

Chemic Changes. - The metabolism of muscle during the contraction is very active. There is an increase in the production of carbon dioxid and in the absorption of oxygen. The muscle changes from an alkaline or neutral to an acid reaction, from the development of sarcolactic acid. The muscle also becomes warmer. The electric changes will be treated of in connection with nerves.

Transmission of the Contraction Wave.-Normally, when a muscle is stimulated by the nerve impulse, the shortening and thickening of the fibers begin at the end organ and travel in opposite directions to the ends of the muscle. This change propagates itself in a wave-like manner, and has been termed the contraction wave. If a stimulus be applied directly to the end of a long muscle, the contraction wave passes along its entire length to the opposite extremity, in virtue of the conductivity of muscular tissue. The rapidity of the propagation varies in different animals-in the frog, from three to four meters a second; in man, from ten to thirteen meters. The length of the wave varies from 200 to 400 millimeters.

Graphic Record of a Muscle Contraction.-The changes in the form of a muscle during contraction and relaxation have been carefully

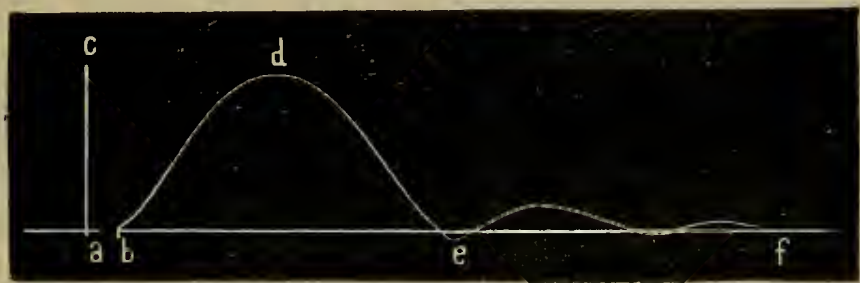

Fig. 4. - Muscle Curve Produced by a Single Induction Shock Applied to A Muscle.-(Landois.)

a-f. Abscissa. a-c. Ordinate. a-b. Period of latent stimulation. b-d. Period of increasing energy. d-e. Period of decreasing energy. e-f. Elastic after-vibrations.

studied by recording the muscle movement by means of an attached lever, the end of which is allowed to rest upon a moving surface. The time relations of all phases of the muscular movement are obtained by placing beneath the lever a pen attached to an electromagnet thrown into action by a 
tuning-fork vibrating in hundredths of a second. A marking lever records simultaneously the moment of stimulation.

Single Contraction.-When a single electric induction shock is applied to a nerve close to the muscle, the latter undergoes a quick pulsation, speedily returning to its former condition. As shown by the muscle curve (see Fig. 4), there is between the moment of stimulation and the beginning of the contraction a short but measurable period, known as the latent period, during which certain chemic changes are taking place preparatory to the exhibition of the muscle movement. Even when the electric stimulus is applied directly to the muscle, a latent period, though shorter, is observable. The duration of this period in the skeletal muscles of the frog has been estimated at 0.01 of a second; but it has been shown by the employment of more accurate methods and the elimination of various external influences to be much less-not more than 0.0033 to 0.0025 of a second.

The contraction follows the latent period. This begins slowly, rapidly reaches its maximum, and ceases. This has been termed the stage of rising or increasing energy. The time occupied in the stage of shortening is about 0.04 of a second, though this will depend on the strength of the stimulus, the load with which the muscle is weighted, and the condition of the muscle irritability.

The relaxation immediately follows the contraction. This takes place at first slowly, after which the muscle rapidly returns to its original length. This is the period of falling or decreasing energy, and occupies about 0.05 of a second. The whole.duration of a muscle contraction occupies, therefore, about 0.1 of a second.

Residual or after-vibrations are frequently seen which are due to changes in the elasticity of the muscle. The amplitude of the contraction depends upon the condition of the muscle, the load, the strength of stimulus, etc.

Contraction of Non-striated Muscle.-The curve obtained by registration of the contraction of non-striated muscle shows that it is similar in many respects to that of the striated muscle, except that the duration of the former is considerably longer than that of the latter.

Action of Successive Stimuli.-If a series of successive stimuli be applied to a muscle, the effect will be different according to the rapidity with which they follow one another. If the second stimulus be applied at the termination of the contraction due to the first stimulus, a second contraction follows, similar in all respects to the first. A third stimulus pro- 
duces a third contraction, and so on until the muscle becomes exhausted. If the second stimulus be applied during either of the two periods of the first contraction, the effects of the two stimuli will be added together and the second contraction will add itself to the first. The maximum contraction is obtained when the second stimulus is applied $\frac{1}{2} \sigma$ of a second after the first.

Tetanus.-When a series of stimuli are applied to a muscle, following one another with median rapidity, the muscle does not get time to relax in ihe intervals of stimulation, but remains in a state of vibratory contraction, which may be regarded as incipient tetanus, or clonus. As the stimulation increases in frequency, the vibrations become invisible, being completely fused together. There is, nevertheless, during the tetanic condition a series of continuous contractions and relaxations taking place. After a varying length of time the muscle becomes fatigued, and notwithstanding the stimulation, begins slowly to elongate. The number of stimuli necessary a second for the production of tetanus varies in different animals-e.g., 2 to 3 for muscles of the tortoise; Io for muscles of the rabbit; 15 to 20 for the frog; 70 to So for birds; 330 to 340 for insects.

A voluntary contraction in man may be regarded as a state of tetanus, for if the curve of a voluntary movement be examined, it will be found to consist of intermittent vibrations. The simplest voluntary movement of a muscle, however rapidly it may take place, lasts longer than a single muscular contraction due to an induction shock. The most rapid voluntary contraction is the result of from 2.5 to 4 stimulations a second, and has a duration of from 0.041 to 0.064 of a second. A continuous voluntary contraction is an incomplete tetanus. The number of stimuli sent to the muscle is, on the average, 16 to 18 for rapid contractions, 8 to I 2 for slow contractions.

The Production of Heat and Its Relation to Mechanical Work. -The transformation of energy which takes" place during a muscle contraction, and which is dependent upon chemic changes occurring at that time, manifests itself as heat and mechanical work. While heat is being evolved continuously during the passive condition of muscles, the amount of heat is largely increased during general muscle contraction. A skeletal muscle of a frog, - e.g., the gastrocnemius,-when removed from the body, shows, after tetanization, an increase in its temperature of from $0.14^{\circ}$ to $0.18^{\circ} \mathrm{C}$, and after a single contraction of from $0.001^{\circ}$ to $0.005^{\circ} \mathrm{C}$. While every muscular contraction is attended by an increase in heat production, 
the amount so produced will vary in accordance with certain conditionse. g., tension, work done, fatigue, circulation of blood, etc.

Tension.-The greater the tension of a muscle, the greater, other conditions being equal, is the amount of heat evolved. When the ends of a muscle are fastened so that no shortening is possible during stimulation, the maximum of heat production is reached. In the tetanic state the great increase in temperature is due to the tension of antagonistic and strongly contracted muscles. The evolution of heat, therefore, bears a relation to the resistance against which the muscle is acting.

Mechanical Work.-If a muscle contracts, loaded by a weight just sufficient to elongate it to its original length, heat is evolved, but no mechanical work is done, all the energy liberated manifesting itself as heat. When the weight which has been lifted is removed from the muscle at the height of contraction, external work is done. In this case the amount of heat liberated is less, owing to the work done, for some of the heat generated is transformed into mechanical motion. According to the law of the conservation of energy, the amount of heat disappearing should correspond in heat units to the number of foot-pounds produced by muscular contraction.

Muscle Sound.-Providing a muscle be kept in a state of tension during its contraction, the intermittent variations of its tension cause the muscle to emit an audible sound. If the muscle be tetanized by induction shocks, the pitch of the sound corresponds with the number of stimuli a second. A voluntary contraction is attended by a tone having a vibration frequency of about thirty-six a second, which is, however, the first overtone of the true muscle tone, which is caused by a contraction frequency of about eighteen a second. This low tone is inaudible, from the small number of vibrations a second.

Muscle Fatigue.-Prolonged or excessive muscular activity is followed by a diminution in the power of producing work and by an increase in the duration of the muscular contractions. Fatigue is accompanied by a feeling of stiffness, soreness, and lassitude, referable to the muscles themselves. In the early stages of muscular fatigue the contractions increase in height and duration, to be followed by a progressive decrease in height, but an increase in duration, until the muscle becomes exhausted. The cause of the fatigue is the production and accumulation of decomposition products, such as phosphoric acid and phosphate of potassium, $\mathrm{CO}_{2}$, etc. A fatigued muscle is rapidly restored by the injection of arterial blood.

Work Done.-Muscles are machines capable of doing a certain amount of work, by which is meant the raising of a weight against gravity or the 
overcoming of some resistance. The work done is calculated by multiplying the weight by the distance through which it is raised. Thus, if a muscle shortens four millimeters and raises 250 grams, it does work equal to I,000 milligram-meters, or one gram-meter. If a muscle contracts without being weighted, no work is done. Equally, when the muscle is overweighted so that it is unable to contract, no work is done. The amount of work a muscle can do will depend upon the area of its transverse section, the length of its fibers, and the amount of the weight. The amount of work a laborer of 70 kilograms weight performs in eight hours averages 105,605 kilogram-meters, or 340.2 foot-tons.

\section{SPECIAL PHYSIOLOGY OF MUSCLES.}

The individual muscles of the axial and appendicular portions of the body are named with reference to their shape, action, structure, etc.-e. $g$., deltoid, flexor, penniform, etc. In different localities a group of muscles having a common function is named in accordance with the kind of motion it produces or gives rise to-e. g., groups of muscles which alternately bend or straighten a joint, or alternately diminish or increase the angular distance between two bones, are known respectively as flexors and extensors; such muscle groups are in association with ginglymus joints. Muscles which turn the bone to which they are attached around its own axis without producing any great change of position are known as rotators, and are in association with the enarthrodial or ball-and-socket joints. Muscles which impart an angular movement of the extremities to and from the median line of the body are termed abductors and adductors.

In addition to the actions of individual groups of muscles in causing special movements in some regions, several groups of muscles are coördinated for the accomplishment of certain definite functions-e. $g$., muscles of respiration, mastication, expression. The coördination of axial and appendicular muscles enables the individual to assume certain postures, such as standing and sitting; to perform various acts of locomotion, as walking, running, swimming, etc.

Levers.-The function or special mode of action of individual muscles can be understood only when the bones with which they are connected are regarded as levers whose fulcra or fixed points lie in the joints where the movement takes place, and when the muscles are considered as sources of power for imparting movement to the levers, with the object of overcoming resistance or raising weights. 
In mechanics, levers of three kinds or orders are recognized, according to the relative positions of the fulcrum or axis of motion, the applied power, and the weight to be moved. (See Fig. 5.).

In levers of the first order the fulcrum, $F$, lies between the weight or resistance, $\mathrm{W}$, and the power of moving force, $\mathrm{P}$. The distance $\mathrm{P}-\mathrm{F}$ is known as the power arm, the distance $\mathrm{W}-\mathrm{F}$ as the weight arm. As an example of this form of lever in the human body may be mentioned :

1. The elevation of the trunk from the flexed position. The axis of movement, the fulcrum, lies in the hip-joint; the weight, that of the trunk, acting as if concentrated at its center of gravity, lies between the shoulders; the power, the contracting muscles attached to the tuberosity of the ischium. The opposite movement is equally one of the first order, but the relative positions of $\mathrm{P}$ and $\mathrm{W}$ are reversed.

2. The skull in its movements backward and forward upon the atlas.

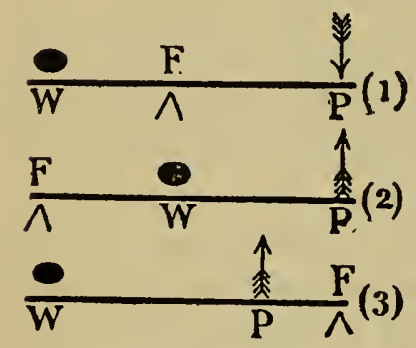

Fig. 5. - The Three Orders OF LeVers.

In levers of the second order the weight lies between the power and the fulcrum. As an illustration of this form of lever may be mentioned :

I. The depression of the lower jaw, in which movement the fulcrum is the temporomaxillary articulation; the resistance, the tension of the elevator muscles; the power, the contraction of the depressor muscles.

2. The raising of the body on the toes$\mathrm{F}$ being the toes, $\mathrm{W}$ the weight of the body acting through the ankle, $P$ the gastrocnemius muscle acting upon the heel bone.

In levers of the third order the power is applied at a point lying between the fulcrum and the weight. As examples of this form of lever may be mentioned :

I. The flexion of the forearm-F being the elbow-joint, $\mathbf{P}$ the contracting biceps and brachialis anticus muscles applied at their insertion, $\mathrm{W}$ the weight of the forearm and hand.

2. The extension of the leg on the thigh.

When levers are employed in mechanics, the object aimed at is the overcoming of a great resistance by the application of a small force acting through a great space, so as to obtain a mechanical advantage. In the mechanism of the human body the reverse generally obtains-viz., the overcoming of a small resistance by the application of a great force acting through a small space. As a result, there is a gain in the extent and 
rapidity of movement of the lever. The power, however, owing to its point of application, acts at a great mechanical disadvantage in many instances, especially in levers of the third order.

Postures.-Owing to its system of joints, levers, and muscles, the human body can assume a series of positions of equilibrium, such as standing and sitting, to which the name posture has been given. In order that the body may remain in a state of stable equilibrium in any posture, it is essential that the vertical line passing through the center of gravity shall fall within the base of support.

Standing is that position of equilibrium in which a line drawn through the center of gravity falls within the area of both feet placed on the ground. This position is maintained :

I. By firmly fixing the head on the top of the vertebral column by the action of the muscles on the back of the neck.

2. By making the vertebral column rigid, which is accomplished by the longissinus dorsi and the quadratus lumborum muscles. This having been accomplished, the center of gravity falls in front of the tenth dorsal vertebra; the vertical line passing through this point falls behind the line connecting both hip-joints. In consequence, the trunk is not balanced on the hip-joints, and would fall backward were it not prevented by the contraction of the rectus femoris muscle and ligaments. At the knees and ankles a similar balancing of the parts above is brought about by the action of various muscles. When the entire body is in the erect or military position, the arms by the sides, the center of gravity lies between the sacrum and the last lumbar vertebra, and the vertical line touches the ground between the feet and within the base of support.

Sitting erect is a condition of equilibrium in which the body is balanced on the tubera ischii, when the trunk and head together form a rigid column. The vertical line passes between the tubera.

Locomotion is the act of transferring the body, as a whole, through space, and is accomplished by the combined action of its own muscles. The acts involved consist of walking, running, jumping, etc.

Walking is a complicated act, involving almost all the voluntary muscles of the body, either for purposes of progression or for balancing the head and trunk, and may be defined as a progression in a forward horizontal direction, due to the alternate action of both legs. In walking, one leg becomes for the time being, the active or supporting leg, carrying the trunk and head; the other, the passive but progressive leg, to become in turn the active leg when the foot touches the ground. Each leg, therefore, is alternately in an active and a passive state. 
Running is distinguished from walking by the fact that, at a given moment, both feet are off the ground and the body is raised in the air.

While the limits of a compend do not permit of a description of the origin, insertion, and mode of action of the individual muscles of the body, it has been thought desirable to call attention to a few of the principal muscles whose function it is to produce special forms of movement, as well as locomotion. (See Fig. 6.) The erect position is largely maintained by the fixation of the spinal column and the balancing of the head upon its upper extremity; the former is accompanied by the erector spince muscle, named from its function and its fleshy continuations, situated on each side of the vertebral column. Arising from the pelvis and lumbar vertebræ, this muscle passes upward, and is attached by its continuations to all the vertebræ. Its action is to extend the vertebral column and to maintain the erect position. The head is balanced upon the top of the vertebral column by the combined action of the trapezius and suboccipital muscles forming the nape of the neck, and by the sterno-cleido-mastoid muscle. This latter muscle arises from the inner third of the clavicle and upper border of the sternum. It is inserted into the temporal bone just behind the ear. Its action is to flex the head laterally and to rotate the face to the opposite side. When both muscles act simultaneously, the head and neck are flexed upon the thorax.

The temporal and masseter muscles, situated at the side of the head, arise respectively from the temporal fossa and the zygomatic arch, and are inserted into the ramus of the lower jaw. Their action is to close the mouth and to assist in mastication. The occipitofrontalis, the orbicularis palpebrarum, and orbicularis oris muscles are largely concerned in wrinkling the forehead, closing the eyes and mouth, and in giving various expressions to the face.

The deltoid is a thick, triangular muscle covering the shoulder-joint. Arising from the outer third of the clavicle, the acromial process, and the spine of the scapula, its fibers converge to be inserted into the humerus just above its middle point. Its action is to elevate the arm through a right angle. Owing to its point of insertion it acts as a lever of the third order, but, notwithstanding the advantageous point of insertion, it acts at a considerable disadvantage, owing to the obliquity of its direction.

The biceps muscle, situated on the anterior aspect of the arm, arises from the upper border of the glenoid fossa and the coracoid process, and is inserted into the radius just beyond the elbow-joint. Its action is to flex and supinate the forearm and to place it in the most favorable position for striking a blow. When the forearm is fixed, it assists in filexing the arm, as in climbing. 


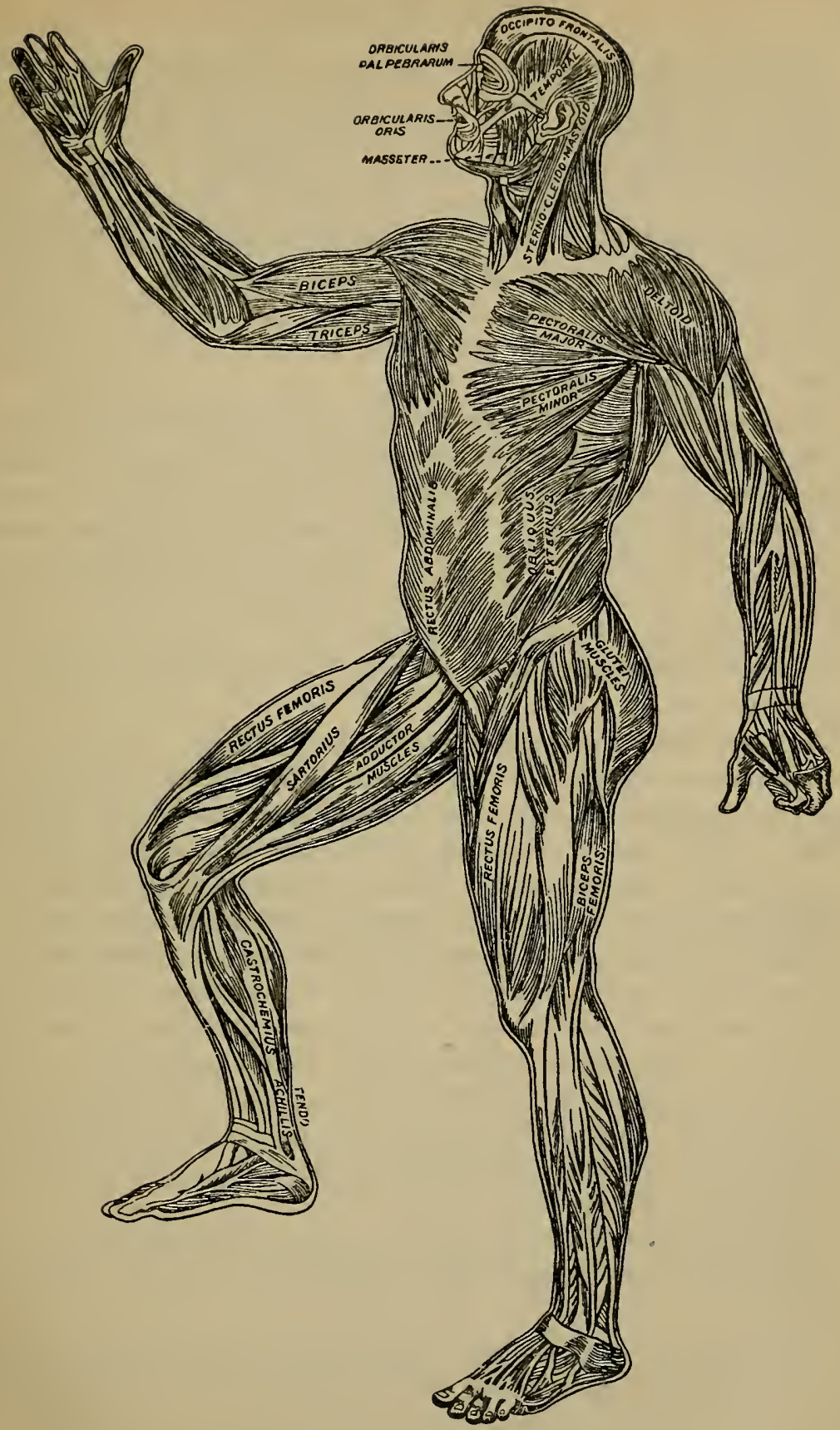

Fig. 6.-Superficial Muscles of the Body. 
The triceps muscle, situated on the back of the arm, arises from the scapula and the posterior surface of the humerus, and is inserted in the olecranon process of the ulna. In its action it directly antogonizes the biceps, namely, extending the forearm. In so doing it acts as a lever of the first order. The short distance between the muscular insertion and the fulcrum causes it to act at a great mechanical disadvantage, but there is a corresponding gain in both speed and range of movement. The muscles of the forearm are very numerous. Their action is to impart to the forearm and hand a variety of movements, such as pronation, supination, flexion, extension, rotation, etc.

The pectoralis major and pectoralis minor muscles form the fleshy masses of the breast. Arising from the inner half of the clavicle, the side of the sternum, and the outer surfaces of the third, fourth, and fifth ribs unteriorly, the muscle-fibers converge to be inserted into the humerus and coracoid process. Their combined action is to adduct, flex, and rotate the arm inward, and to draw the scapula downward and forward, movements necessary to the folding of the arms across the chest.

The rectus abdominis and the obliquus externus assist in forming the abdominal walls.

The glutei muscles are three in number, are arranged in layers, and form the fleshy masses known as the buttocks. They arise from the side of the pelvis and are attached to the femur in the neighborhood of the great trochanter. Their action is to extend the hips, to raise the body from the stooping position, and to assist in walking by firmly holding the pelvis on the thigh while the opposite leg is advanced in the forward direction.

The rectus femoris, with its associates, the rectus internus and rectus externus and the crureus, forms the fleshy mass on the anterior surface of the thigh. The former arises from the anterior part of the ilium, the latter from the femur. Their common tendon, which is united to the patella, is continued as the ligamentum patellæ, which is attached to the upper part of the tibia. The action of this muscular group is to extend the leg, to flex the thigh, and to raise the entire weight of the body, as in changing from the sitting to the erect position.

The biceps femoris muscle, situated on the outer and posterior aspect of the thigh, arises from the tuber ischii, and is inserted into the head of the fibula.

The semimembranosus and the semitendinosus muscles, situated on the inner and posterior aspect of the thigh, are inserted into the head of the tibia. Their combined action is to extend the hips and to flex the knee. Acting from below, they assist in raising the body from the stooping position. 
The gastrocnemius muscle forms the enlargement known as the calf of the leg. It arises by two heads from the condyles of the femur. Its tendon, the tendo Achillis, is inserted into the posterior surface of the heel bone. Its action is to extend the foot and to raise the weight of the body in walking and running. On the front of the leg are numerous musclese. g., tibialis anticus, peroneus longus, etc., the action of which is to flex the foot and to antagonize the gastrocnemius.

\section{PHYSIOLOGY OF NERVE TISSUE.}

The nerve tissue, which unites and coördinates the various organs and tissues of the body and brings the individual into relationship with the external world, is arranged anatomically into two systems, termed the cerebro-spinal and the sympathetic.

The cerebro-spinal system consists of :

I. The brain and spinal cord, contained within the cavities of the cranium and the spinal column respectively, and

2. The cranial and spinal nerves.

- The sympathetic system consists of

I. A double chain of ganglia situated on each side of the spinal column and extending from the base of the skull to the tip of the coccyx.

2. Various collections of ganglia situated in the head, face, thorax, abdomen, and pelvis. All these ganglia are united by an elaborate system of intercommunicating nerves, many of which are connected with the cerebro-spinal system.

\section{HISTOLOGY OF NERVE TISSUE.}

The Neuron.-The nerve tissue has been resolved by the investigations of modern histologists into a single morphologic unit, to which the term neuron has been applied. The entire nervous system has been shown to be but an aggregate of an infinite number of neurons, each of which is histologically distinct and independent. Though having a common origin, as shown by embryologic investigations, they have acquired a variety of forms in different parts of the nervous system in the course of development. The old conception that the nervous system consisted of two distinct histologic elements, nerve-cells and nerve-fibers, which differed not only in their mode of origin, but also in their properties, their relation to each other, and their functions, has been entirely disproved. 
The neuron, or neurologic unit, is histologically a nerve-cell, the surface of which presents a greater or less number of processes in varying degrees of differentiation. As represented in figure 7 , the neuron may be said to consist of : (I) The nerve-cell, neurocyte, or corpus ; (2) the axon, or nerve process ; (3) the end tufts, or terminal branches. Though these three main histologic features are everywhere recognizable, they exhibit a variety of secondary features in different situations in accordance with peculiarities of function. Though the nerve-cell and the nerve-fiber are but part of the same neuron, it is convenient at present to describe them separately.

The Nerve-cell.-The nerve-cell, or body of the neuron, presents a variety of shapes and sizes in different portions of the nervous system. Originally ovoid in shape, it has acquired, in course of development, peculiarities of form which are described as pyramidal, stellate, pear-shaped, spindle-shaped, etc. The size of the cell varies considerably, the smallest having a diameter of not more than $\frac{1}{3000}$ of an inch, the largest not more than $\frac{1}{40} \overline{0}$ of an inch. Each cell consists of granular, striated protoplasm, containing a distinct vesicular nucleus and a well-defined nucleolus. A cell membrane has not been observed. From the surface of the adult cell portions of the protoplasm are projected in various directions, which portions, rapidly dividing and subdividing, form a series of branches, termed dendrites or dendrons. In some situations the ultimate branches of the dendrites present short lateral processes, known as lateral buds, or gemmules, which impart to the branches a feathery appearance. This characteristic is common to the cells of the cortex, of the cerebrum, and of the cerebellum. The ultimate branches of the dendrites, though forming an intricate feltwork, never anastomose with one another, nor unite with dendrites of adjoining cells. According to the number of axons, nerve-cells are classified as monaxonic, diaxonic, polyaxonic. Most of the cells of the nervous system of the higher vertebrates are monaxonic. In the ganglia of the posterior or dorsal roots of the spinal and cranial nerves, however, they are diaxonic. In this situation the axons, emerging from opposite poles of the cell, either remain separate and pursue opposite directions, or unite to form a common stem, which subsequently divides into two branches, which then pursue opposite directions. (See Fig. 7.) The nerve-cell maintains its own nutrition, and presides over that of the dendrites and the axon as well. If the latter be separated in any part of its course from the cell, it speedily degenerates and dies.

The axon, or nerve process, arises from a cone-shaped projection from 
the surface of the cell, and is the first outgrowth from its protoplasm. At a short distance from its origin it becomes markedly differentiated from the dendrites which subsequently develop. It is characterized by a sharp, regular outline, a uniform diameter, and a hyaline appearance. In structure, the axon appears to consist of fine fibrilla embedded in a clear, protoplasmic substance. Shäfer advocates the view that the fibrillæ are

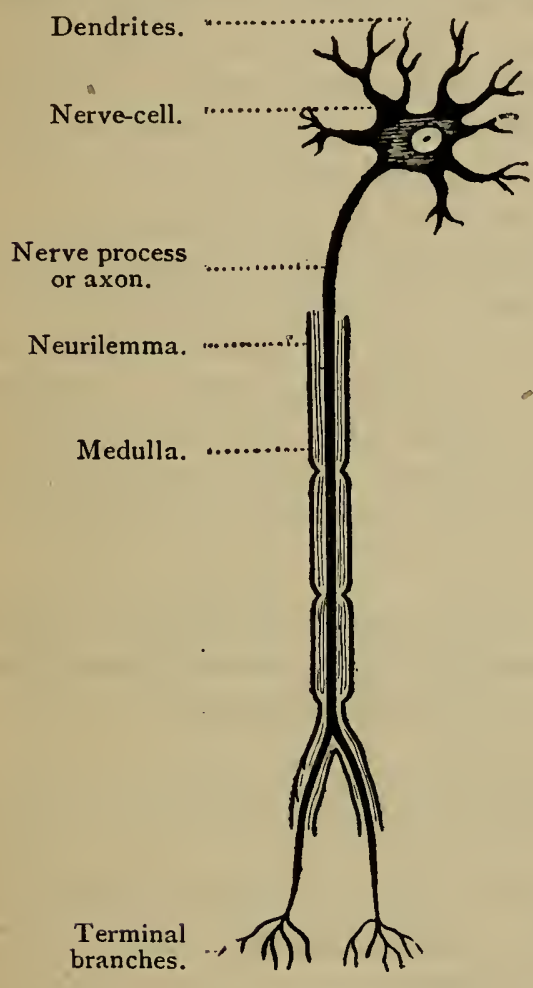

A

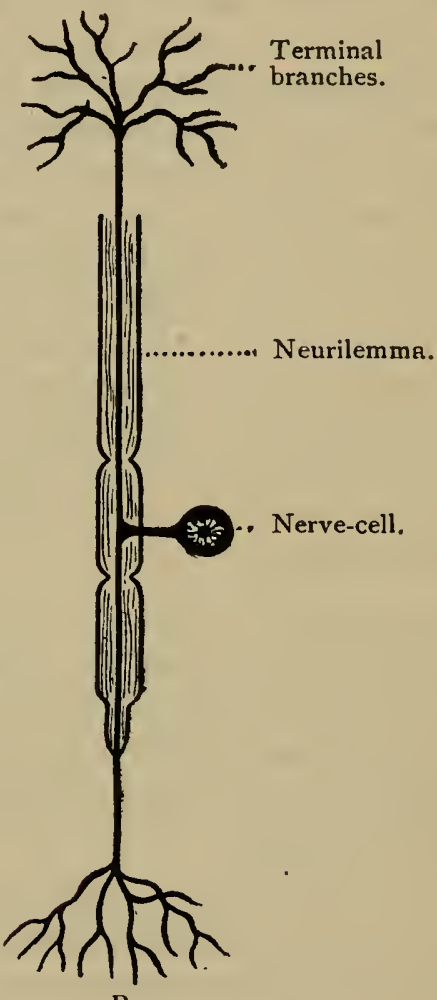

B

FIG. 7 .

A. Efferent neuron. B. Afferent neuron.

exceedingly fine tubes filled with fluid. The axon varies in length from a few millimeters to $100 \mathrm{~cm}$. In the former instance the axon, at a short distance from its origin, divides into a number of branches, which form an intricate feltwork in the neighborhood of the cell. In the latter instance the axon continues for an indefinite distance as an individual structure. In its course, however, especially in the central nervous system, it gives off a number of collateral branches, which possess all its histologic features. 
The long axons seem to bring the body of the cell into direct relation with peripheral organs, or with more or less remote portions of the nervous system, thus constituting association or commissural fibers.

The more or less elongated axon becomes invested, as a rule, at a short distance from the cell with nucleated oblong cells, which subsequently become modified and constitute a medullary or myelin sheath. This is invested by a thin, cellular membrane-the neurilemma. These three structures thus constitute what is known as a medullated nerve-fiber. In the central nervous system the outer sheath is frequently absent. In the sympathetic system the myelin is frequently absent, though the axon is inclosed by the neurilemma, thus constituting a non-medullated nerve-fiber.

The end tufts or terminal organs are formed by the splitting of the axon into a number of filaments, which remain independent of one another and are free from the medullary investment. The histologic peculiarities of the terminal organs vary in different situations, and in many instances are quite complex and characteristic. In peripheral organs, as muscles, glands, blood-vessels, skin, mucous membrane, the tufts are in direct organic connection with their cellular elements. In the central nervous system the tufts are in more or less intimate relation with the dendrites of adjacent neurons.

Nerve-fibers.-The axons with their secondary investments together constitute the nerve-fibers, and according as they possess or do not possess the medullary sheath, they may be divided into two groups-viz., medullated and non-medullated fibers.

Medullated Nerve-fibers.-These consist for the most part of three distinct structures :

I. An external investing sheath, tubular in shape, termed the neurilemma.

2. An intermediate semifluid substance-the medulla or myelin.

3. An internal dark thread-the axis-cylinder.

The neurilemma is a thin, transparent, homogeneous membrane closely adherent to the medulla. Owing to its colorless appearance, it can be seen only with difficulty in the recent condition. When treated with various reagents, it becomes distinct. Physically, it is quite resistant and elastic. Its function is doubtless that of a protective agent to the structures within.

The medulla, myelin, or white substance of Schreann completely fills the neurilemma and closely invests the axis-cylinder. In the recent condition the medulla is clear, homogeneous, semifluid, and highly refracting. In composition it is oleaginous. When the nerve is treated with various 
reagents which alter its composition, the medulla becomes opaque and imparts to the nerve a white, glistening appearance. The function of the medulla is quite unknown.

At intervals of about seventy-five times its diameter the medullated nerve-fiber undergoes a remarkable diminution in size, due to an interruption of the medullary substance, so that the neurilemma lies directly on the axis-cylinder. These constrictions, or nodes of Ranvier, taking their name from their discoverer, occur at regular intervals along the course of the nerve, separating it into a series of segments. The portion between the nodes is termed the internodal segment. It has been suggested that in consequence of the absence of the myelin at these nodes, a free exchange of nutritive material and decomposition products can take place between the axis-cylinder and the surrounding plasma.

The axis-cylinder, or axon, the direct outgrowth of the nerve-cell, is the most essential element of the nerve-fiber, as it alone is uniformly continuous throughout. In the natural condition it is transparent and invisible; but when treated with proper reagents, it presents itself as a pale, granular, flattened band, more or less solid and somewhat elastic. It is albuminous in composition. With high magnification the axis presents a longitudinal striation, indicating a fibrillar structure. The fibrillæe appear to be united by an intervening cement substance.

Non-medullated Nerve-fibers.-These consist, for the most part, only of the axis-cylinder, though in some portions of the nervous system a neurilemma is also present. Though much less abundant than the former variety, they are distributed largely throughout the nervous system, but are particularly abundant in the sympathetic system. Owing to the absence of a medulla, they present a rather pale or grayish appearance.

Structure of Nerve Trunks.-After their emergence from the brain and spinal cord, the nerve-fibers become bound together, by connective tissue, into the form of continuous bundles, which connect the brain and cord with all the remaining structures of the body. The bundles are technically known as nerve trunks or nerves. Each nerve is invested by a thick layer of lamellated connective tissue, known as the epineurizm. A transverse section of a nerve shows (see Fig. 8) that it is made up of a number of small bundles of fibers, each of which possesses a separate investment of connective tissue-the perineurium. IVithin this membrane the nerve-fibers are supported by a fine stroma-the endoneurium. After pursuing a longer or shorter course, the nerve trunk gives off branches, which interlace very freely with neighboring branches, forming plexuses, 
the fibers of which are distributed to associated organs and regions of the body. From their origin to their termination, however, nerve-fibers retain their individuality, and never become blended with adjoining fibers.

As nerves pass from their origin to their peripheral terminations, they give off a number of branches, each of which becomes invested with a lamellated sheath-an offshoot from that investing the parent trunk. This division of nerve bundles and sheath continues throughout all the branchings down to the ultimate nerve-fibers, each of which is surrounded by a

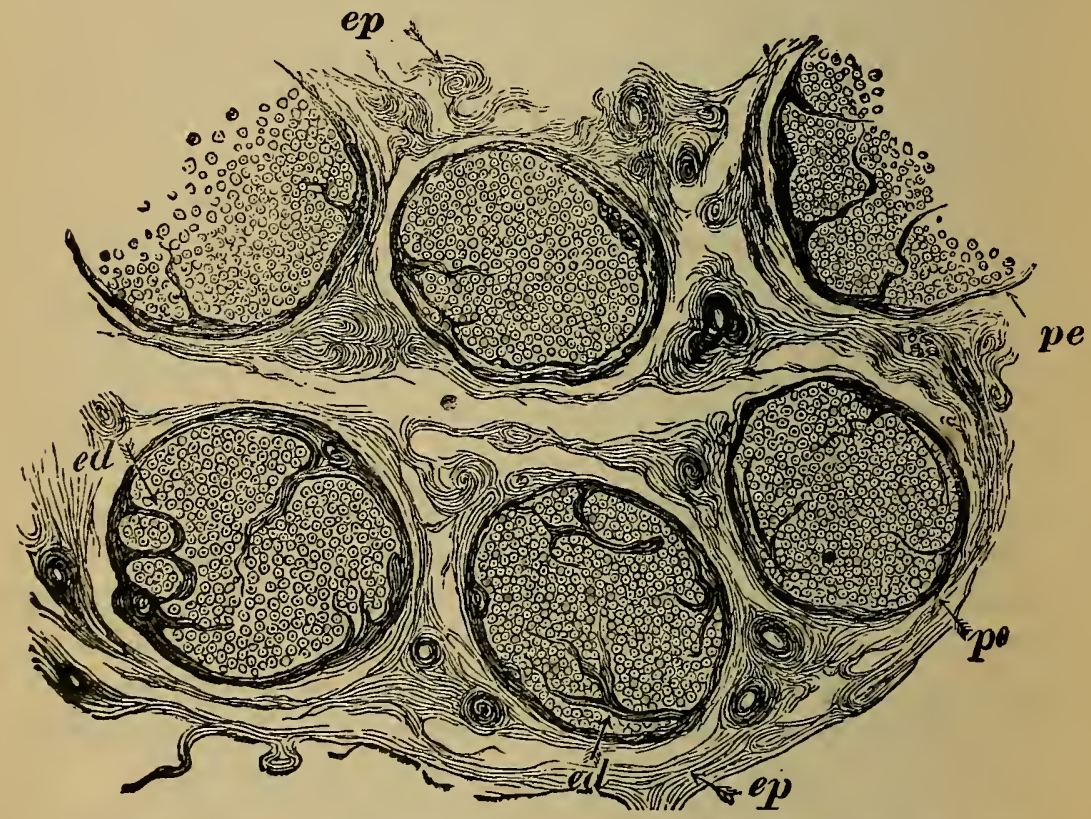

Fig. 8.-Transverse Section of a Nerve (Median).

ep Epineurium. pe. Perineurium. ed. Endoneurium.

sheath of its own, consisting of a single layer of endothelial cells. This delicate transparent membrane, the sheath of Henle, is separated from the nerve-fiber by a considerable space, in which is contained lymph destined for the nutrition of the fiber. Near their ultimate terminations the nervefibers themselves undergo division, so that a single fiber may give origin to a number of branches, each of which contains a portion of the parent axiscylinder and myelin. 


\section{CLASSIFICATION OF NERVES.}

Nerves are channels of communication between the brain and spinal cord, on the one hand, and the muscles, glands, blood-vessels, skin, mucous membrane, viscera, etc., on the other. Some of the nerve-fibers serve for the transmission of nerve energy or nerve impulses from the brain and spinal cord to certain peripheral organs, and so increase or retard their activities; others serve for the transmission of nerve energy from certain peripheral organs to the brain and spinal cord, which gives rise to sensations or other modes of nerve activity. The former are termed efferent or centrifugal nerves; the latter are termed afferent or centripetal nerves.

The efferent nerves may be classified, in accordance with the characteristic form of activity to which they give rise, into several groups, as follows :

I. Muscuiar or motor nerves, those which convey nerve energy or nerve impulses to muscles and give rise to muscular contraction.

2. Glandular or secretory nerves, those which convey nerve impulses to glands, and cause the formation of the secretion peculiar to the gland.

3. Vascular or vaso-motor nerves, those which convey nerve impulses to blood-vessels, and cause, either by stimulation or inhibition of the mechanism of their walls, a contraction (vaso-constrictors) or dilatation (vasodilators) of the vessel.

4. Inhibitory nerves, those conveying nerve impulses that cause a slowing or complete cessation of the rhythmic action of organs.

5. Accelerator nerves, those conveying impulses that cause an increase in the rhythmic action of certain organs.

The afferent nerves may also be classified, in accordance with the character of the sensations or other modes of nerve activity to which they give rise, into several groups, as follows :

I. Sensorifacient nerves, those conveying nerve impulses that give rise in the brain to conscious sensations. They may be subdivided into-

(a) Nerves of special sense-e. g., olfactory, optic, auditory, gustatory, tactile, thermal, sensory, muscle-those which give rise to olfactory, optic, auditory, gustatory, tactile, thermic, painful, and muscle sensations.

(b) Nerves of general sense-e. g., the visceral afferent nerves-those which give rise normally to vague and scarcely perceptible sensations, such as the general sensations of well-being or discomfort, hunger, thirst, fatigue, sex, want of air, etc. 
2. Reflex nerves, those which convey nerve impulses to the nerve centers and cause a discharge and transmission of nerve impulses outward through efferent nerves to muscles, glands, or blood-vessels, and thus influence their activity. It is quite probable that one and the same nerve may subserve both sensational and reflex action, owing to the collateral branches which are given off from the posterior roots as they ascend the posterior column of the cord.

3. Inhibitory nerves, those which are capable reflexly of retarding or inhibiting the activity of either nerve centers or peripheral organs.

The Terminal Endings of Nerves.-The efferent nerves, as they approach their ultimate terminations, lose both the neurilemma and medullary sheath. The axis-cylinder then divides into a number of tufts or branches, which become directly and intimately connected with the tissue. cells. The particular mode of termination varies in different situations. These terminations are generally spoken of as "end organs"

In the skeletal muscles the nerve-fiber loses both neurilemma and myelin sheath at the point where it comes into contact with the muscle-fiber. After penetrating the sarcolemma, the axis-cylinder breaks up into small branches with bulbous extrenities, forming the so-called "motor plate," which rests directly on a disc of granular material containing oval, vesicular nuclei. Each muscle-fiber possesses an individual end-plate.

In the visceral muscles the terminal nerve-fibers form a plexus around the muscle-fibers, and become organically connected with them. In the glands the nerve-fibers have been traced directly to their secreting cells. The exact mode of their termination and connection with the cells has not been clearly determined.

The afferent nerves, as they approach their peripheral terminations, become connected in like manner with end organs, which, in some instances, are extremely complex, such as those found in the eye (retina), the internal ear, the nose, and the tongue. (A consideration of these end organs will be found in the chapters devoted to the organs of which they form a part.) The end organs of the skin and mucous membranes present a variety of forms, and may be classified as follows :

I. Free endings in the epithelium of the skin, mucous membrane, and cornea.

2. Tactile cells of Merkel in the epidermis.

2. Tactile corpuscles in the papillæ of the true skin.

4. Pacinian corpuscles found attached to the nerves of the hands and feet, to the intercostal nerves, and to nerves in other situations.

5. End bulbs of Krause in the conjunctiva, penis, clitoris, etc. 
The end organs of the afferent nerves are specialized, highly irritable structures placed between the nerve-fibers and the surface of the body. They are especially adapted for the reception of those external forces technically known as stimuli, and for the liberation of energy capable of exciting the nerve-fiber to activity.

\section{Relation of Peripheral Nerves to the Central Nervous System.-} The nerves in connection with the spinal cord are thirty-one in number on each side and have two roots of origin, an anterior and a posterior, which arise from the anterior and posterior surfaces of the cord respectively. They are more properly termed ventral and dorsal roots. The dorsal roots present, near their entrance into the cord, an enlargement termed a ganglion. Beyond the spinal canal these two roots unite to form the ordinary spinal nerve. Some of the nerves in connection with the base of the brain also present a ganglionic enlargement, and may, therefore, be regarded physiologically as dorsal nerves, while others may be regarded as ventral nerves. .

Experimentally, it has been determined that the anterior or ventral roots contain all the efferent fibers, the posterior or dorsal roots all the afferent fibers. The proofs in support of this view are as follows:

Stimulation of the ventral roots produces :

I. Convulsive movements of muscles.

2. The formation of a secretion in glands.

3. Changes in the caliber of blood-vessels.

4. Inhibition of the rhythmic activity of certain organs.

Divisions of these roots is followed by :

I. Loss of muscular movement (paralysis of motion).

2. Cessation of secretion.

3. Cessation of vascular changes.

Stimulation of the dorsal roots causes :

I. Reflex activities.

2. Conscious sensations.

3. Inhibition of the rhythmic activity of certain organs.

Division of these roots is followed by :

I. Loss of reflex activities, and

2. Loss of sensation in all parts to which they are distributed.

The ventral roots are, therefore, efferent in function, transmitting nerve impulses from the nerve centers to the periphery. The dorsal roots are afferent in function, transmitting nerve impulses from the general peripbery to the nerve centers. 
Development and Nutrition of Nerves.-The efferent nervefibers, which constitute some of the cranial nerves and all the ventral roots of the spinal nerves, have their origin in cells located in the gray matter beneath the aqueduct of Sylvius, beneath the floor of the fourth ventricle and in the anterior horns of the gray matter of the spinal cord. These cells are the modified descendants of independent, oval, pear-shaped cells - the neuroblasts-which migrate from the medullary tube. As they approach the surface of the cord their axons are directed toward the ventral surface, which eventually they pierce. Emerging from the cord,

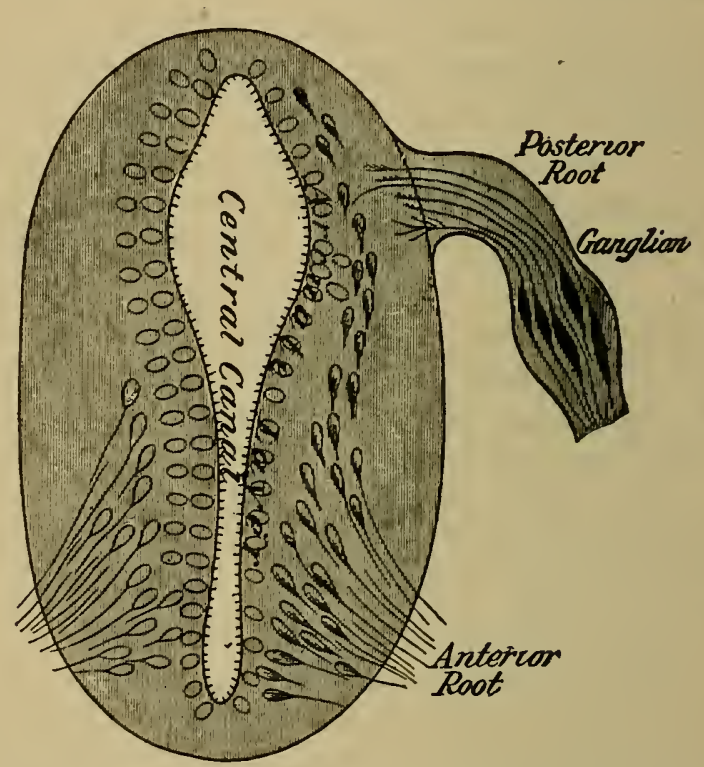

Fig. 9.-Diagram Showing the Mode of Origin of the Ventral and Dorsal. Roots.

the axions continue to grow, and become invested with the myelin sheath and neurilemma, thus constituting the ventral roots.

The afferent nerve-fibers, which constitute some of the cranial nerves and all the dorsal roots of the spinal nerves, develop outside of the central nervous system and only subsequently become connected with it. (See Fig. 9.) At the time of the closure of the medullary tube a band or ridge of epithelial tissue develops near the dorsal surface, which, becoming segmented, moves outward and forms the rudimentary spinal ganglia. The cells in this situation develop two axons, one from each end of the cell, which 
pass in opposite directions, one toward the spinal cord, the other toward the periphery. In the adult condition the two axons shift their position, unite, and form a T-slıaped process, after which a division into two branches again takes place. In the ganglia of all the sensoricranial and sensorispinal nerves the cells have this histologic peculiarity.

Nerve Degeneration.-If any one of the cranial or spinal nerves be divided in any portion of its course, the part in connection with the periphery in a short time exhibits certain structural changes, to which the term degeneration is applied. The portion in connection with the brain or cord retains its normal condition. The degenerative process begins simultaneously throughout the entire course of the nerve, and consists in a disintegration and reduction of the medulla and axis-cylinder into nuclei, drops of myelin, and fat, which in time disappear through absorption, leaving the neurilemma intact. Coincident with these structural changes there is a progressive alteration and diminution in the excitability of the nerve. Inasmuch as the central portion of the nerve, which retains its connection with the nerve-cell, remains histologically normal, it has been assumed that the nerve-cells exert over the entire course of the nerve-fibers a nutritive or a trophic influence. This idea has been greatly strengthened since the discovery that the axis-cylinder, or the axon, has its origin in and is a direct outgrowth of the cell. When separated from the parent cell, the fiber appears to be incapable of itself of maintaining its nutrition.

The relation of the nerve-cells to the nerve-fibers, in reference to their nutrition, is demonstrated by the results which follow section of the ventral and dorsal roots of the spinal nerves. If the anterior root alone be divided, the degenerative process is confined to the peripheral portion, the central portion remaining normal. If the posterior root be divided on the peripheral side of the ganglion, degeneration takes place only in the peripheral portion of the nerve. If the root be divided between the ganglion and the cord, degeneration takes place only in the central portion of the root. From these facts it is evident that the trophic centers for the ventral and dorsal roots lie in the spinal cord and spinal nerve ganglia, respectively, or, in other words, in the cells of which they are an integral part. The structural changes which nerves undergo after separation from their centers are degenerative in character, and the process is usually spoken of, after its discoverer, as the Wallerian degeneration.

When the degeneration of the efferent nerves is completed, the structures to which they are distributed, especially the muscles, undergo an atrophic or fatty degeneration, with a change or loss of their irritability. This is, 
apparently, not to be attributed merely to inactivity, but rather to a loss of nerve influences, inasmuch as inactivity merely leads to atrophy and not to degeneration.

Reactions of Degeneration. - In consequence of the degeneration and changes in irritability which occur in nerves and muscles when separated, either experimentally or as the result of disease, the response of these structures to the induced and the make-and-break of the constant currents differs from that observed in the physiologic condition. The facts observed under the application of these two forms of electricity are of the greatest importance in the diagnosis and therapeutics of the precedent lesions. The principal difference of behavior is observed in the muscles, which exhibit a diminished or abolished excitability to the induced current, while at the same time manifesting an increased excitability to the constant current; so much so is this the case that a closing contraction is just as likely to occur at the positive as at the negative pole. This peculiarity of the muscle response is termed the reaction of degeneration. The synchronous diminished excitability of the nerves is the same for either current. The term "partial reaction of degeneration" is used when there is a normal reaction of the nerves, with the degenerative reaction of the muscles. This condition is observed in progressive muscular atrophy.

Reflex Action.-Inasmuch as many of the muscle movements of the body, as well as the formation and discharge of secretions from glands, variations in the caliber of blood-vessels, inhibition and acceleration in the activity of various organs, are the result of stimulations of the terminal organs of afferent nerves, they are termed, for convenience, reflex actions, and, as they take place independently of the brain or of volitional impulses, they are also termed involuntary actions. As many of the processes to be described in succeeding chapters are of this character, requiring for their performance the coöperation of several organs and tissues associated through the intermediation of the nervous system, it seems advisable to consider briefly, in this connection, the parts involved in a reflex action, as well as their mode of action. As shown in figure ro, the necessary structures are as follows:

1. A sentient surface, skin, mucous membrane, sense organ, etc.

2. An afferent nerve.

3. An emissive cell, from which arises

4. An efferent nerve, distributed to a responsive organ, as

5. Muscle, gland, blood-vessel, etc.

Such a combination of structures constitutes a reflex mechanism or arc, 
the nerve portion of which is composed of but two neurons-an afferent and an efferent. An arc of this simplicity would of necessity subserve but a simple movement. The majority of reflex activitics, however, are extremely complex, and inrolve the coöperation and coördination of a number of structures frequently situated at distances more or less remote from one another. This implies that a number of neurons are associated in function. The afferent neurons are brought into relation with the dendrites of the efferent neurons by the end tufts of the collateral branches,

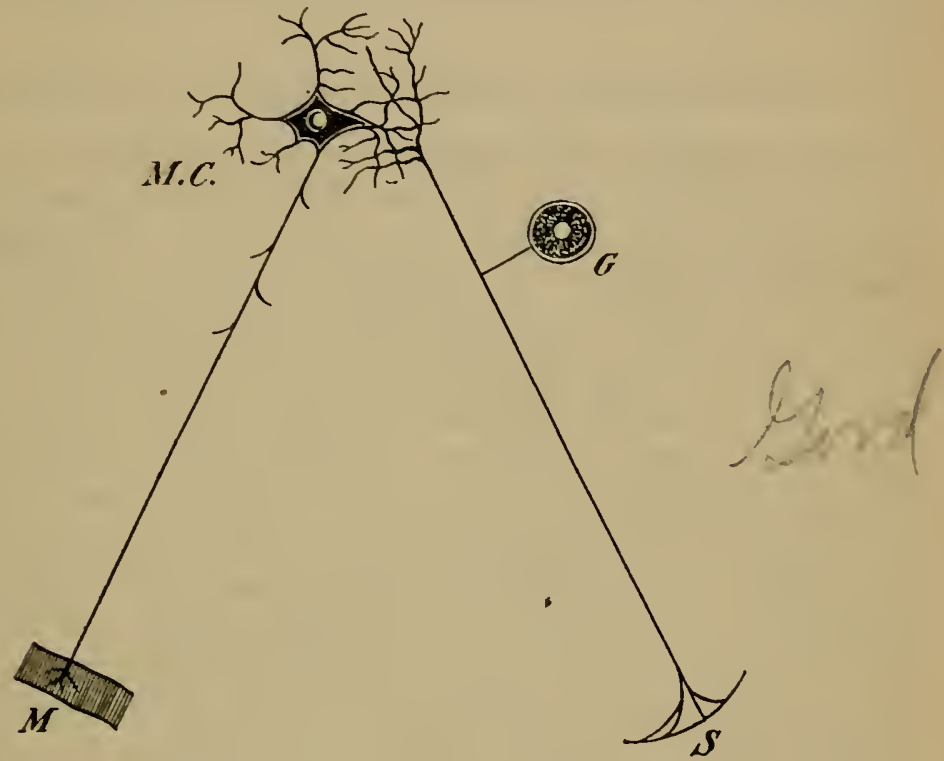

Fig. io.-Diagram Illustrating Reflex Action.-(Kirke.)

S. Sentient surface from which proceeds the afferent nerve. MT. C. Motor or emissive cell giving origin to efferent nerve which terminates in $M$. M. Motor organ. $G$. Ganglion cell on afferent nerve

which may extend for some distance up and down the cord before passing into the various segments.

For the excitation of a reflex action it is essential that the stimulus applied to the sentient surface be of an intensity sufficient to develop in the terminals of the afferent nerve a series of nerve impulses, which, traveling inward, will be distributed to and received by the dendrites of the emissive or motor cell. With the reception of these impulses there is apparently a disturbance of the equilibrium of its molecules, a liberation of energy, and, in consequence, a transmission outward of impulses through the 
efferent nerve to muscle, gland, or blood-vessel, separately or collectively, with the production of muscular contraction, glandular secretion, vascular dilatation or contraction, etc. The reflex actions take place, for the most part, through the spinal cord and medulla oblongata, which, in virtue of their contained centers, coördinate the various organs and tissues concerned in the performance of the organic functions. The movements of mastication; the secretion of saliva; the muscular, glandular, and vascular phenomena of gastric and intestinal digestion; the vascular and respiratory movements ; the mechanism of micturition, etc., are illustrations of reflex activity.

\section{PHYSIOLOGIC PROPERTIES OF NERVES.}

\section{Nerve Irritability or Excitability and Conductivity.-These terms} are employed to express that condition of a nerve which enables it to develop and to conduct nerve impulses from the center to the periphery, from the periphery to the center, in response to the action of stimuli. A nerve is said to be excitable or irritable as long as it possesses these capabilities or properties. For the manifestation of these properties the nerve must retain a state of physical and chemic integrity ; it must undergo no change in structure or chemic composition. The irritability of an efferent nerve is demonstrated by the contraction of a muscle, by the secretion of a gland, or by a change in the caliber of a blood-vessel, whenever a corresponding nerve is stimulated. The irritability of an afferent nerve is demonstrated by the production of a sensation or a reflex action whenever it is stimulated. The irritability of nerves continues for a certain period of time after separation from the nerve centers and even after the death of the animal, varying in different classes of animals. In the warm-blooded animals, in which the nutritive changes take place with great rapidity, the irritability soon disappears-a result due to disintegrative changes in the nerve, caused by the withdrawal of the blood-supply. In cold-blooded animals, on the contrary, in which the nutritive changes take place relatively slowly, the irritability lasts, under favorable conditions, for a considerable tine. Other tissues besides nerves possess irritability, that is, the property of responding to the action of stimuli-e.g., glands and muscles, which respond by the production of a secretion or a contraction.

Independence of Tissue Irritability.-The irritability of nerves is distinct and independent of the irritability of muscles and glands, as shown by the fact that it persists in each a variable length of time after their histologic connections have been impaired or destroyed by the introduction of various chemic agents into the circulation. Curara, for example, induces a 
state of complete paralysis by nodifying or depressing the conductivity of the end organs of the nerves just where they come in contact with the muscles without impairing the irritability of either nerve trunks or muscles. Atropin induces complete suspension of glandular activity by impairing the terminal organs of the secretory nerves just where they come into relation with the gland-cells, without destroying the irritability of either gland or nerve.

Stimuli of Nerves.-Nerves do not possess the power of spontaneously generating and propagating nerve impulses; they can be aroused to activity only by the action of an extraneural stimulus. In the living condition the stimuli capable of throwing the nerve into an active condition act for the most part on either the central or peripheral end of the nerve. In the case of motor nerves the stimulus to the excitation, originating in some molecular disturbance in the nerve-cells, acts upon the nerve-fibers in connection with them. In the case of sensory or afferent nerves the stimuli act upon the peculiar end organs with which the sensory nerves are in connection, which in turn excite the nerve-fibers. Experimentally, it can be demonstrated that nerves can be excited by a sufficiently powerful stimulus applied in any part of their extent.

Nerves respond to stimulation according to their habitual function; thus, stimulation of a sensory nerve, if sufficiently strong, results in the sensation of pain ; of the optic nerve, in the sensation of light; of a motor nerve, in contraction of the muscle to which it is distributed; of a secretory nerve, in the activity of the related gland, etc. It is, therefore, evident that peculiarity of nervous function depends neither upon any special construction or activity of the nerve itself, nor upon the nature of the stimulus, but entirely upon the peculiarities of its central and peripheral end organs.

Nerve stimuli may be divided into-

1. General stimuli, comprising those agents which are capable of exciting a nerve in any part of its course.

2. Special stimuli, comprising those agents which act upon nerves only through the intermediation of the end organs.

\section{General stimuli:}

1. Mechanical : as from a blow, pressure, tension, puncture, etc.

2. Thermal: heating a nerve at first increases and then decreases its excitability.

3. Chemic: sensory nerves respond somewhat less promptly than motor nerves to this form of irritation.

4. Electric : either the constant or interrupted current. 
5. The normal physiologic stimulus :

(a) Centrifugal or efferent, if proceeding from the center toward the periphery.

(b) Centripetal or afferent, if in the reverse direction. Special stimuli:

I. Light or ethereal vibrations acting upon the end organs of the optic nerve in the retina.

2. Sound or atmospheric undulations acting upon the end organs of the auditory nerve.

3. Heat or vibrations of the air upon the end organs in the skin.

4. Chemic agencies acting upon the end organs of the olfactory and gustatory nerves.

Nature of the Nerve Impulse.-As to the nature of the nerve impulse generated by any of the foregoing stimuli either generai or special, but little is known. It has been supposed to partake of the nature of a molecular disturbance, a combination of physical and chemical processes attended by the liberation of energy, which propagates itself from molecule to molecule. Judging from the deflections of the galvanometer needle it is probable that when the nerve impulse makes its appearance at any given point it is at first feeble but soon reaches a maximum development after which it speedily declines and disappears. It may, therefore, be graphically represented as a wave-like movement with a definite length and time duration. Under strictly physiological conditions the nerve impulse $\mathrm{p}_{i}$ asses in one direction only; in efferent nerves from the center to the periphery, in afferent nerves from the periphery to the center. Experimentally, how ever, it can be demonstrated that when a nerve impulse is aroused in the course of a nerve by an adequate stimulus it travels equally well in both directions from the point of stimulation. When once started the impulse is confined to the single fiber and does not diffuse itself to fibers adjacent to it in the same nerve trunk.

Rapidity of Transmission of Nerve Force.-The passage of a nervous impulse, either from the brain to the periphery or in the reverse direction, requires an appreciable period of time. The velocity with which the impulse travels in human sensory nerves has been estimated at about 190 feet a second, and for motor nerves at from 100 to 200 feet a second. The rate of movement is, however, somewhat modified by temperature, cold lessening and heat increasing the rapidity ; it is also modified by electric conditions, by the action of drugs, the strength of the stimulus, etc. The rate of transmission through the spinal cord is considerably slower 
than in nerves, the average velocity for voluntary motor impulses being only 33 feet a second, for sensitive impressions 40 feet, and for tactile impressions 140 feet a second.

Electric Currents in Muscles and Nerves.-If a muscle or nerve be divided and non-polarizable electrodes be placed upon the natural longitudinal surface at the equator, and upon the transverse section, electric currents are observed with the aid of a delicate galvanometer. The direction of the current is always from the positive equatorial surface to the negative transverse surface. The strength of the current increases or diminishes according as the positive electrode is moved toward or from the equator. When the electrodes are placed on the two transverse ends of a nerve, an axial current will be observed the direction of which is opposite to that of the normal impulse of the nerve.

The electromotive force of the strongest nerve-current has been estimated to be equal to the 0.026 of a Daniell battery; the force of the current of the frog muscle, about 0.05 to 0.08 of a Daniell.

Negative Variation of Currents in Muscles and Nerves.-If a muscle or nerve be thrown into a condition of tetanus, it will be observed that the currents undergo a diminution or negative variation, a change which passes along the nerve in the form of a wave and with a velocity equal to the rate of transmission of the nerve impulse. The wave-length of a single negative variation has been estimated to be eighteen millimeters, the period of its duration being from 0.0005 to 0.0008 of a second.

It is asserted by Hermann that perfectly fresh, uninjured muscles and nerves are devoid of currents, and that the currents observed are the result of molecular death at the point of section, this point beconing negative to the equatorial point. He applies the term "action currents" to the currents obtained when a muscle is thrown into a state of activity.

Electric Properties of Nerves. - When a galvanic current is made to flow along a motor nerve from the center to the periphery, from the positive to the negative pole, it is known as the direct, descending, or centrifugal current. When it is made to flow in the reverse direction, it is known as the inverse, ascending, or ientripetal current.

The passage of a direct current enfeebles the excitability of a nerve; the passage of the inverse current increases it. The excitability of a nerve may be exhausted by the repeated applications of electricity; when thus exhausted, it may be restored by repose, or by the passage of the inverse current if the nerve has been exhausted by the direct current, or vice versâ. 
During the actual passage of a feeble constant current, in either direction, neither pain nor muscular contraction is ordinarily manifested; if the current be very intense, the nerve may be disorganized and its excitability destroyed.

Electrotonus. - The passage of a direct galvanic current through a portion of a nerve excites in the parts beyond the electrodes a condition of electric tension, or electrotonus, during which the excitability of the nerve is decreased near the anode or positive pole, and increased near the cathode or negative pole; the increase of excitability in the catelectrotonic areathat nearest the muscle-being manifested by a more marked contraction of the muscle than the normal when the nerve is irritated in this region. The passage of an inverse galvanic current excites the same condition of electrotonus; the diminution of excitability near the anode, the anelectrotonic area,-that now nearest the muscle,-being manifested by a less marked contraction than the normal when the nerve is stimulated in this region. Between the electrodes is a neutral point, where the catelectrotonic area emerges into the anelectrotonic area. If the current be a strong one, the neutral point approaches the cathode; if weak, it approaches the anode.

When a nervous impulse passes along a nerve, the only appreciable effect is a change in its electric condition, there being no change in its temperature, chemic composition, or physical condition. The natural nerve-currents, which are always present in a living nerve as a result of its nutritive activity, in great part disappear during the passage of an impulse, undergoing a negative variation.

Law of Contraction.-If a feeble galvanic current be applied to a recent and excitable nerve, contraction is produced in the muscles only upon the making of the circuit with both the direct and inverse currents.

If the current be moderate in intensity, the contraction is produced in the muscle, both upon the making and breaking of the circuit, with both the direct and inverse currents.

If the current be intense, contraction is produced only when the circuit is made with the direct current, and only when it is broken with the inverse current.

\section{FOODS AND DIETETICS.}

During the functional activity of every organ and tissue of the body the living material of which it is composed-the protoplasm-undergoes more or less disintegration. Through a series of descending chemic stages it is reduced to a number of simpler compounds, which are of no further value to the body, and which are in consequence eliminated by the various elim- 
inating or excretory organs-the lungs, kidneys, skin, liver. Among these compounds the more important are carbon dioxid, urea, and uric acid. Many other compounds, inorganic as well as organic, are also eliminated in the water discharged from the body, in which they are held in solution. Coincident with this disintegration of the tissues there is an evolution or disengagement of energy, particularly in the form of heat.

In order that the tissues may regain their normal composition and thus be enabled to continue in the performance of their functions, they must be supplied with the same nutritive materials of which their protoplasm originally consisted-viz., water, inorganic salts, proteids, sugar, fat. These materials are furnished by the blood during its passage through the capillary blood-vessels. The blood is a reservoir of nutritive material in a condition to be absorbed, organized, and transformed into new living tissue.

Inasmuch as the loss of material from the body daily, which is very great, is compensated for under other forms by the blood, it is evident that this fluid would rapidly diminish in volume were it not restored by the introduction of new and corresponding materials. As soon as the blood volume falls to a certain point, the sensations of hunger and thirst arise, which in a short time lead to the necessity of taking food.

In addition to the direct appropriation of food by the tissues it is highly probable that an indefinite amount undergoes oxidation and disintegration without ever becoming an integral part of the tissues, and thus directly contributes to the production of heat.

Inanition or Starvation.-If these nutritive principles be not supplied in sufficient quantity, or if they are withheld entirely, a condition of physiologic decay is established, to which the term inanition or starvation is applied. The phenomena which characterizes this pathologic process are as follows - viz., hunger, intense thirst, gastric and intestinal uneasiness and pain, muscle weakness and emaciation, a diminution in the quantity of carbon dioxid exhaled, a lessening in the amount of urine and its constituents excreted, a diminution in the volume of the blood, an exhalation of a fetid odor from the body, vertigo, stupor, delirium, and at times conrulsions, a fall of bodily temperature, and, finally, death from exhaustion.

During starvation the loss of different tissues, before death occurs, averages $\frac{4}{10}$, or 40 per cent., of their weight.

Those tissues which lose more than 40 per cent. are : Fat, 93.3 ; blood, 75 ; spleen, 71.4 ; pancreas, 64.1 ; liver, 52 ; heart, 44.8 ; intestines, 42.4; muscle, 42.3. Those which lose less than 40 per cent. are: The muscular coat of the stomach, 39.7 ; pharynx and esophagus, 34.2 ; skin, 33.3 ; kidneys, 31.9 ; respiratory apparatus, 22.2 ; bones, 16.7 ; eyes, 10 ; nervous system, $\mathbf{1} .9$.

The fat entirely disappears, with the exception of a small quantity which 
remains in the posterior portion of the orbits and arount the kidneys. The blood diminishes in volume and loses its nutritive properties. The muscles undergo a marked diminution in volume and become soft and flabby. The nervous system is last to suffer, not more than two per cent. disappearing before death occurs.

The appearances presented by the body after death from starvation are those of anemia and great emaciation; almost total absence of fat ; bloodlessness; a diminution in the volume of the organs; an empty condition of the stomach and bowels, the coats of which are thin and transparent. There is a marked disposition of the body to undergo decomposition, giving rise to a very fetid odor.

The duration of life after a complete deprivation of food varies from eight to thirteen days, though life can be maintained much longer if a quantity of water be obtained. The water is more essential under these circumstances than the solid matters, which can be supplied by the organism itself.

The different alimentary or nutritive principles which are appropriated by the tissues, and which are contained within the various articles of food, belong to both the organic and inorganic groups and chemic compounds, and may be classified according to their composition as follows :

\section{CLASSIFICATION OF ALIMENTARY PRINCIPLES.}

\section{Proteid Group.-Nitrogenized, C, O, H, N, S, P.}

Principle.

Myosin, .

Vitellin, albumin, .

Fibrin, globulin,

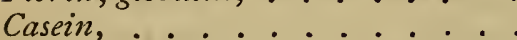

Gluten, . . . . . . . .

Vegetable albumin, .......

Legumin, . . . . . . . . .

Gelatin, . . .

2. Oleaginous Group.- $\mathrm{C}, \mathrm{O}, \mathrm{H}$.

Animal fats and oils, . . . . .

Stearin, olein,

Palmitin, fatty acids, . . . . .

3. Carbohydrate Group.- C, O, H.

Saccharose, or cane-sugar, . . . .

Dextrose, or glucose, . . . . . .

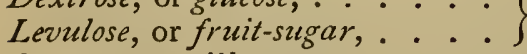

Lactose, or milk-sugar, . . . .

Maltose, . . . . . . . .

Starch,

Glycogen,
Where Found.

Flesh of animals.

Yolk of egg, white of egg.

Blood contained in meat.

Milk, cheese.

Grain of wheat and other cereals.

Soft, growing vegetables.

Peas, beans, lentils, etc.

Bones.

Found in the adipose tissue of animals, seeds, grains, nuts, fruits, and other vegetable tissues.

Sugar-cane.

Fruits.

Milk.

Malt, malt foods.

Cereals, tuberous roots, and leguminous plants.

Liver, muscles. 
4. Inorganic Group.-Water; sodium and potassium chlorids; sodium calciunı, magnesium, and potassium phosphates; calcium carbonate; and iron.

5. Vegetable Acid Group.-Malic, citrıc, tartaric, and other acids, found principally in fruits.

6. Accessory Foods. - Tea, coffee, alcohol, cocoa, etc.

The proteid principles of the food, after undergoing digestion and conversion into peptones, are absorbed and transformed into the form of proteids characteristic of the blood plasma and the lymph. Of the proteids thus brought into relation with the living protoplasm, a small percentage only is utilized in the repair of its substance. This is known as tissue proteid. A large percentage circulating among and permeating the tissues is acted upon by them directly, and reduced to simpler compounds without ever becoming a part of the tissue itself. This is known as circulaingr proteid. In the process of tissue metabolism all the proteids suffer disintegration, and give rise to the production of some carbon-holding compound, probably fat, and some nitrogen-holding compounds which eventually produce urea. The intermediate stages are possibly represented by glycin, creatin, uric acid, etc. An excess of proteids in the food is followed by their decomposition, by the pancreatic juice, into leucin and tyrosin, which, by the agency of the liver, are converted into urea. The disintegration of the proteids is attended by the disengagement of heat : they thus contribute to the energy of the body.

The oleaginous principles, after digestion, are absorbed into the blood, from which they rapidly disappear. It is probable that a portion of the fat enters directly into the composition of living protoplasm, out of which it again emerges at some subsequent stage in the form of small drops which make their appearance in the protoplasmic cells of the connective areolar tissue, thus giving rise to the adipose tissue. Another portion probably undergoes direct oxidation.

The carbohydrate principles, after digestion, are absorbed as dextrose and temporarily stored up in the liver as glycogen. The intermediate stages which sugar passes through and the combinations into which it enters between its absorption and its elimination are but imperfectly understood. That it contributes to the accumulation of fat is probable, though it is doubtful if it is ever converted into fat. A large percentage of the sugar absorbed is at once oxidized. The reduction of fat and sugar to carbon dioxid and water, under which forms they are eliminated from the body, is accompanied by the disengagement of a large quantity of heat. 
Water is present in all the fluids and solids of the body. It promotes the absorption of new material from the alimentary canal; it holds the various ingredients of the blood, lymph, and other fluids in solution; it hastens the absorption of waste products from the tissues, and promotes their speedy elimination from the body.

Sodium chlorid is present in all parts of the body to the extent of 110 $\mathrm{gm}$. The average amount eliminated daily is $15 \mathrm{gm}$. Its necessity as an article of diet is at once apparent. Taken as a condiment, it imparts sapidity to the food, excites the flow of the digestive fluids, promotes the absorption and assimilation of the albumins, influences the passage of nutritive material through animal membranes, and furnishes the chlorin for the free hydrochloric acid of the gastric juice. In some unknown way it favorably promotes the activity of the general nutritive process.

The potassizum salts are also essential to the normal activity of the nutritive process. When deprived of these salts, animals become weak and emaciated. When given in small doses, they increase the force of the heart-beat, raise the arterial pressure, and thus increase the action of the circulation of the blood.

The calcium phosphate and carbonate are utilized in imparting solidity to the tissues, more especially the bones and teeth. Many articles of food contain these salts in quantities sufficient to restore the amount lost daily.

The vegetable acids increase the secretions of the alimentary canal, and are apt, in large amounts, to produce flatulence and diarrhea. After entering into combination with bases to form salts, they stimulate the action of the kidneys and promote a greater elimination of all the urinary constituents. In some unknown way they influence nutrition; when deprived of these acids, the individual becomes scorbutic.

The accessory foods, coffee and tea, when taken in moderation, overcome the sense of fatigue and mental unrest consequent on excessive physical and mental exertion. Coffee increases the action of the intestinal glands and acts as a laxative. After absorption, its active principle, caffein, stimulates the action of the heart, raises the arterial pressure, and excites the action of the brain. Tea acts as an astringent, owing to the tannic acid it contains. One effect of the tannic acid is to coagulate the digestive ferments and to interfere with the activity of the digestive process.

Alcohol, when introduced into the system in small quantities, undergoes oxidation and contributes to the production of force, and is thus far a food. It excites the gastric glands to increased secretion, improves the digestion, accelerates the action of the heart, and stimulates the activities of the nerve centers. In zymotic diseases, and in all cases of depression of the 
vital powers, it is most useful as a restorative agent. When taken in excessive quantities, it is elininated by the lungs and kidneys. The metamorphosis of the tissue is retarded, the elimination of urea and carbonic acid is lessened, the temperature is lowered, the muscular powers are impaired, and the resistance to depressing external influences is diminished. When taken throughout a long period of time, alcohol impairs digestion, produces gastric catarrh, and disorders the secreting power of the hepatic cells. It also diminishes the muscular power and destroys the structure and composition of the cells of the brain and spinal cord. The connective tissue of the body increases in amount, and, subsequently contracting, gives rise to sclerosis.

A proper combination of various alimentary principles is essential for healthy nutrition, no one class being capable of maintaining life for any definite length of time.

The albuminous food in excess promotes the arthritic diathesis, manifesting itself as gout, gravel, etc.

The oleaginous food in excess gives rise to the bilious diathesis, while a deficiency of it promotes the scrofulous.

The farinaceous food when long continued in excess, favors the rheu. matic diathesis by the development of lactic acid.

The quantities of the different nutritive materials which are required daily for the growth and repair of the tissues and for the evolution of heat have been variously estimated by different observers. The following table shows the average diet scale of Vierordt, and the amount of waste products to which it would give rise :

\section{COMPARISON OF THE INgEsta AND Egesta.}

\begin{tabular}{|c|c|c|c|c|c|}
\hline Ingesta. & & & & esta. & \\
\hline $\begin{array}{l}\text { Proteids, . . } \\
\text { Fat, }\end{array}$ & I 20 & rams. & $\begin{array}{l}\text { Urea, } \\
\text { Inorganic salts }\end{array}$ & 40 & ams \\
\hline $\begin{array}{l}\text { Fat, } \cdot \cdots \cdot \\
\text { Starch, : }\end{array}$ & $\begin{array}{r}90 \\
330\end{array}$ & “" & $\begin{array}{l}\text { Inorganic salts } \\
\text { Feces, . . . }\end{array}$ & $\begin{array}{r}32 \\
104\end{array}$ & "6 \\
\hline Inorganic salts, & $3^{2}$ & "6 & Carbon dioxid & 800 & 66 \\
\hline Water, . . & 2,800 & 66 & Water, . . . & 3,096 & “ \\
\hline xygen, . & 700 & “" & Total, & $\overline{4,072}$ & “" \\
\hline Total, . & $.4,072$ & "6 & & & \\
\hline
\end{tabular}

Other estimates as to the amount of the organic substances required daily are as follows :

\section{Ranke.}

Proteid, . . Ioo

Fat, . . 100

Starch, . . 240
Voit.

I 18

50

500
Moleschott.

I 30 grams.

84
404


The Energy of the Animal Body. - The food consumed daily not only repairs the loss of material from the body, but also furnishes the energy to replace that which is expended daily in the shape of heat and motion. All the energy of the body can be traced to the chemic changes going on in the tissues, and more particularly to those changes involved in the oxidation of the foods.

The amount of heat yielded by any given food principle can be determined by burning it to carbon dioxid and water, and ascertaining the extent to which it will, when so liberated, raise the temperature of a given volume of water. This amount of heat may be expressed in gram degrees of heat -i.e., calories or kilogrammeters of work. A calorie is the amount of heat required to raise the temperature of one gram of water one degree Centigrade, or one kilogram of water one degree Centigrade. A kilogrammeter of work is the amount of heat energy required to raise a weight of one kilogram a distance of one meter.

The following estimates give, approximately, the number of calories produced when the food is reduced within the body to urea, carbon dioxid, and water :

I gram of proteid yields 4, I 24 kilogram calories.

I “

The total number of kilogram calories yielded by any given diet scale can be readily determined by multiplying the preceding factors by the quantities of material consumed. The diet scale of Ranke, for example, yields the following amount:

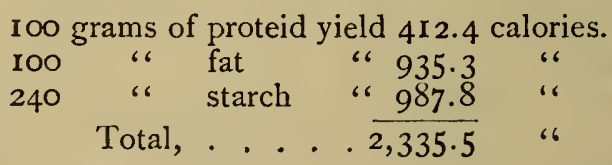

It has also been determined experimentally that one gram of proteid, one gram of fat, and one gram of starch, when completely oxidized, will yield energy sufficient to perform $\mathrm{I}, 850,3,84 \mathrm{I}$, and $\mathrm{I}, 567$ kilogrammeters of work, respectively.

The total energy of the Ranke diet scale can be easily calculated $-e \cdot g$. .

IOO grams of proteid yield 185,000 kilogrammeters.

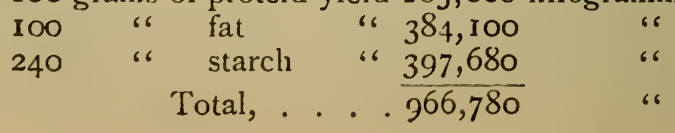


It will be thus seen that the food consumed daily yields $2,335,000 \mathrm{gram}$ calories, or 2,335 kilogram calories, which can be translated into its mechanical equivalent, 966,780 kilogrammeters of work.

The amount of food required in twenty-four hours is estimated from the total quantity of carbon and nitrogen excreted from the body in twenty-four hours, these two elements representing the waste or destruction of the carbonaceous and nitrogenized compounds. It has been determined by experimentation that about 4,600 grains of carbon and about 300 grains of nitrogen are eliminated from the body daily, the ratio being about 15 to $\mathbf{I}$. That the body may be kept in its normal condition, a proper proportion of carbonaceous (bread) to nitrogenized (meat) food should be observed in the diet.

The method of deternining the proper amounts of both kinds of food is as follows :

$$
\text { I,000 grs. of bread ( } 2 \text { oz.) contain } 300 \text { grs. C and Io grs. N. }
$$

To obtain the requisite amount of nitrogen from bread, 30,000 grains, or about four pounds, containing 9,000 grains of carbon and 300 of nitrogen, would have to be consumed. On such a diet there would be a large excess of carbon, which would be undesirable. On a meat diet the reverse obtains :

I, 000 grs. of meat ( 2 oz.) contain 100 grs. C and 30 grs. N.

To obtain the requisite amount of carbon from meat, 45,000 grains, or about $61 / 2$ pounds, containing 4,500 grains of carbon and 1,350 grains of nitrogen would have to be consumed. Under such circumstances there would arise an excess of nitrogen in the system, which would be equally undesirable and injurious. By combining these two articles, however, in proper proportion, the requisite amounts of carbon and nitrogen can be obtained without any excess of either-e. g. :

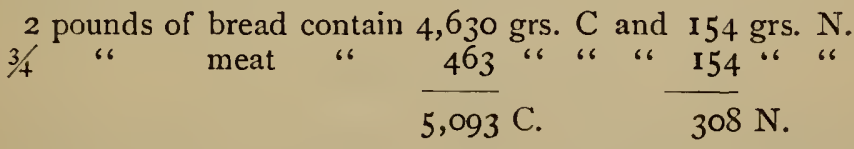

The amount of carbon and nitrogen necessary to compensate for the loss to the system daily would be contained in the foregoing amount of food. As about $3 \frac{1}{2}$ ounces of oil or butter are consumed daily, the quantity of bread can be reduced to 19 ounces. In the quantities of bread and meat just mentioned there are 4.2 ounces albumin, 9.3 sugar and starch. 
The alimentary principles are not introduced into the body as such, but are combined in proper proportions to form compound substances, termed foods, - e. g., bread, milk, eggs, meat, etc., - the nutritive value of each depending upon the extent to which these principles exist.

The following tables show the average composition of various articles of food:

COMPOSITION OF ANIMAL FOODS.

\begin{tabular}{|c|c|c|c|c|c|c|c|c|}
\hline$I_{N} 100$ & PART: & & Beef. & VEAL. & Mutton. & Pork. & Fowl. & Frsh. \\
\hline Water, & . . & . & 76.25 & 77.82 & 75.59 & 72.57 & 70.80 & $79 \cdot 30$ \\
\hline Proteid, & . . & - & 20.24 & 19.86 & I 7. I I & I9.3I & 22.70 & 18.30 \\
\hline Fat,. . & $\cdot \cdot$ & - & I. 68 & 0.82 & 5.47 & 5.82 & 4. IO & 0.70 \\
\hline Carbohyc & drates & & $0.5^{\circ}$ & 0.80 & 0.60 & 060 & I. 20 & 0.90 \\
\hline Salts, . & . . & $\cdot$ & I. $3^{8}$ & 0.70 & I 23 & 1.70 & I. 20 & O.So \\
\hline
\end{tabular}

COMPOSITION OF VEGETABLE FOODS.

\begin{tabular}{|c|c|c|c|c|c|c|}
\hline In IOO PARTS. & Beans. & Peas. & $\begin{array}{l}\text { PotA- } \\
\text { TOES }\end{array}$ & Turnips. & Cabbage. & $\begin{array}{l}\text { Aspara- } \\
\text { GUS. }\end{array}$ \\
\hline Water, . . & I 3.74 & 14.99 & $75 \cdot 47$ & 89.42 & 89.97 & $93 \cdot 75$ \\
\hline Proteid, . . . & 23.21 & 22.85 & 1.95 & I. 35 & I. 89 & I. 79 \\
\hline Fat, . . . & 2.14 & 1.79 & 0.15 & 0.18 & 0.20 & 0.25 \\
\hline Carbohydrates, & 53.67 & $5^{2.36}$ & 20.69 & $7 \cdot 3^{6}$ & 4.87 & 2.63 \\
\hline Cellulose, & 3.69 & 5.43 & 0.76 & 0.94 & 1.84 & 1.04 \\
\hline Salts, . . . & $3 \cdot 55$ & $2.5^{8}$ & 0.98 & 0.75 & 1.23 & 0.54 \\
\hline
\end{tabular}


ICOMPOSITION OF CEREAL FOODS.

\begin{tabular}{|c|c|c|c|c|c|c|}
\hline In IOO PARTS. & WHEAT. & RYE. & BARLEY. & OATS. & Conn. & Rice. \\
\hline Water, . . & $13 \cdot 5^{6}$ & I 2.65 & I 3.77 & 12.37 & I3. 10 & I3. I 2 \\
\hline Proteid, & 12.35 & 12.55 & II. I 4 & 10.41 & 9.85 & 7.88 \\
\hline Fat, . . . & I.75 & I.97 & 2. 16 & 5.23 & $4 \cdot 57$ & 0.85 \\
\hline Carbohydrates, & 67.90 & 67.95 & 64.93 & 57.78 & 68.42 & 76.55 \\
\hline Cellulose, . . & $2.6_{3}$ & 3.00 & $5 \cdot 3^{I}$ & I I, I9 & 2.50 & 0.55 \\
\hline Salts, . . . & I. $8 \mathrm{I}$ & 1.88 & 2.69 & 3.02 & 1.56 & I.05 \\
\hline
\end{tabular}

\section{DIGESTION.}

Digestion is a physical and chemic process by which the food introduced into the alimentary canal is liquefied and its nutritive principles are transformed by the digestive fluids into new substances capable of being absorbed into the blood.

The digestive apparatus consists of the alimentary canal and its appendages-viz., teeth ; salivary, gastric, and intestinal glands ; liver ; and pancreas.

Digestion may be divided into seven stages: prehension, mastication, insalivation, deglutition, gastric and intestinal digestion, and defecation.

Prehension, the act of conveying food into the mouth, is accomplished by the hands, lips, and teeth.

\section{MASTICATION.}

Mastication is the trituration of the food, and is accomplished by the teeth and lower jaw under the influence of muscular contraction. When thoroughly divided, the food presents a larger surface for the solvent action of the digestive fluids, thus aiding the general process of digestion.

The teeth are thirty-two in number, sixteen in each jaw, and divided into four incisors or cutting teeth, two canines, four bicuspids, and six 
molars or grinding teeth; each tooth consists of a crown covered by enamel, a neck, and a root surrounded by the crusta petrosa and embedded in the alveolar process; a section through a tooth shows that its substance is made of dentine, in the center of which is the pulp cavity containing blood-vessels and nerves.

The lower jaw is capable of making a downward and an upward, a lateral and an anteroposterior movement, dependent upon the construction of the temporomaxillary articulation.

The jaw is depressed by the contraction of the digastric, geniohyoid, mylohyoid, and platysma myoides muscles; elevated by the temporal, masseter, and internal pterygoid muscles; moved laterally by the alternate contraction of the external pterygoid muscles; moved anteriorly by the pterygoid, and posteriorly by the united actions of the grniohyoid, mylohyoid, and posterior fibers of the temporal muscles.

The food is kept between the teeth by the intrinsic and extrinsic muscles of the tongue from within, and the orbicularis oris and buccinator muscles from without.

The movements of mastication, though originating in an effort of the will and under its control, are, for the most part, of an automatic or reflex character, taking place through the medulla oblongata and induced by the presence of food within the mouth. The nerves and nerve-centers involved in this mechanism are shown in the following table:

\section{NERVOUS CIR LE OF MASTICATION.}

Afferent or Excitor Nerves.

I. Lingual branch of 5 th pair.

2. Glossopharyngeal.
Efferent or Motor Nerves.

I. $3 \mathrm{~d}$ branch of 5 th pair.

2. Hypoglossal.

3. Facial.

The impressions made upon the terminal filaments of the sensory nerves are transmitted to the medulla; motor impulses are here generated which are transmitted through motor nerves to the muscles involved in the movements of the lower jaw. The medulla not only generates motor impulses, but coördinates them in such a manner that the movements of mastication may be directed toward the accomplishment of a definite purpose.

\section{INSA LIVATION.}

Insalivation is the incorporation of the food with the saliva secreted by the parotid, submaxillary, and sublingual glands; the parotid saliva, thin and watery, is poured into the mouth through Steno's duct; the sub- 
maxillary and sublingual salivas, thick and viscid, are poured into the mouth through Wharton's and Bartholin's ducts.

In their minute structure the salivary glands resemble one another. They belong to the racemose variety, and consist of small sacs or vesicles, which are the terminal expansions of the smallest salivary ducts. Each vesicle or acinus consists of a basement membrane surrounded by blood-vessels and lined with epithelial cells. In the parotid gland the lining cells are granular and nucleated; in the submaxillary and sublingual glands the cells are large, clear, and rontain a quantity of mucigen. During and after secretion very remarkeble changes take place in the cells lining the acini, which are in some way connected with the essential constituents of the salivary fluids.

In a living serous gland,-e.g., parotid,-during rest, the secretory cells lining the acini of the gland are seen to be filled with fine granules, which are often so abundant as to obscure the nucleus and enlarge the cells until the lumen of the acinus is almost obliterated. (See Fig. II.) When the gland begins to secrete the saliva, the granules disappear from the outer
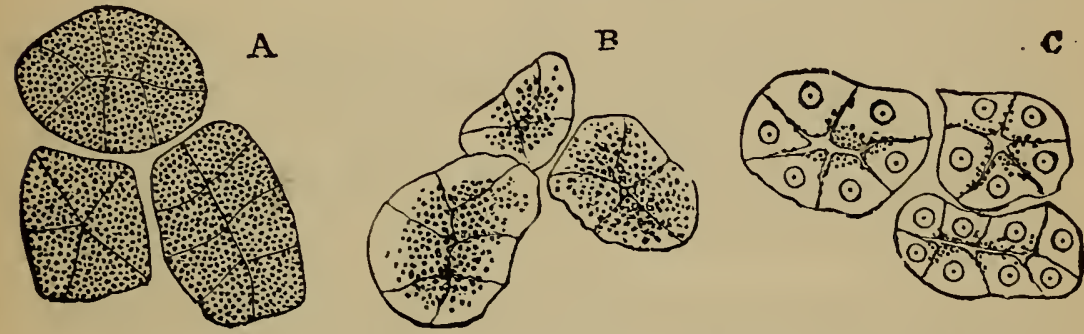

Fig. ir,-Cells of the Alyeoli of a Serous or Watery Salivary Gland.(Yeo's "Text-Book of Physiology.")

A. After rest. B. After a short period of activity. C. After a prolonged period of activity.

boundary of the cells, which then become clear and distinct. At the end of the secretory activity the cells have been freed of granules and have become smaller and more distinct in outline. It would seem that the granular matter is formed in the cells during the period of rest and discharged into the ducts during the activity of the gland.

In the mucous glands- $e . g .$, submaxillary and sublingual-the changes that occur in the cells are somewhat different. (See Fig. 12.) During the intervals of digestion the cells lining the gland are large, clear, and highly refractive, and contain a large quantity of mucigen. After secretion has taken place the cells exhibit a marked change. The mucigen cells have disappeared, and in their place are cells which are small, dark, and 

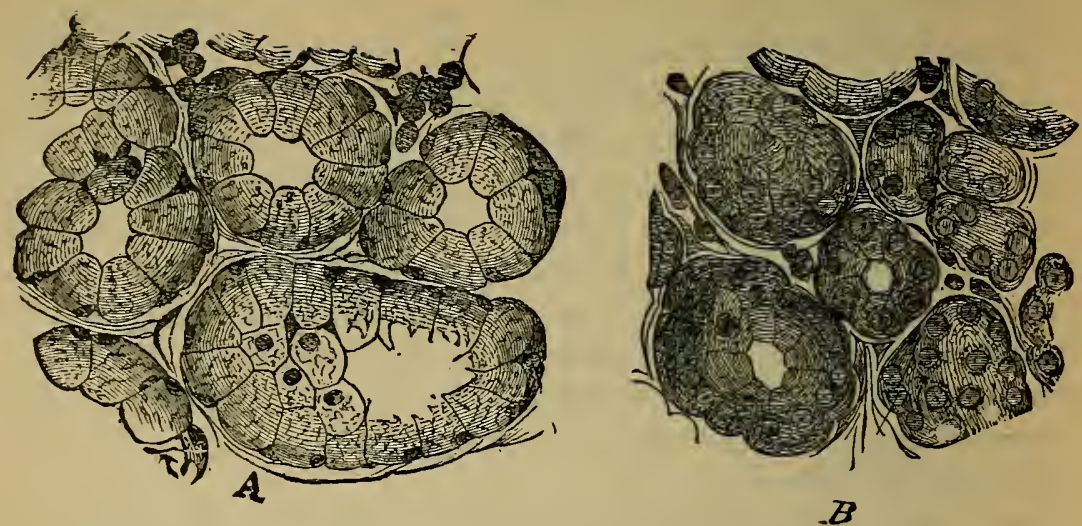

Fig. J2.-Section of a Mucous Gland.-(Lavdozusky.)

A. In a state rest. B. After it has been for some time actively secreting.

composed of protoplasm. - It would appear that the cells, during rest, elaborate the mucigen, which is discharged into the tubules during secretory activity, to become part of the secretion.

Saliva is an opalescent, slightly viscid, alkaline fluid, having a specific gravity of 1.005. Microscopic examination reveals the presence of salivary corpuscles and epithelial cells. Chemically it is composed of water, proteid matter, a ferment (ptyalin), and inorganic salts. The amount secreted in twenty-four hours is about $2 \frac{1}{2} \mathrm{lbs}$. Its function is twofold :

I. Physical.- Softens and moistens the food, agglutinates it, and facilitates swallowing.

2. Chemic.-Converts starch into sugar. This action is due to the presence of the organic ferment, ptyalin. Ptyalin is an amorphous nitrogenized substance, which can be precipitated from the saliva by calcium phosphate. Its power of converting starch into sugar is manifested most decidedly at the temperature of the living body and in a slightly alkaline medium. The conversion of starch into sugar takes place through several stages, the nature of which depends upon the structure of the starch granule. This consists of two portions, a stroma of cellulose and a contained material, granulose, which is the more abundant and important of the two. When subjected to the action of boiling water, the starch granule swells up and bursts, forming a viscid, opalescent mass of starch paste. If saliva be now added to this paste and kept at a temperature of $\mathrm{IO}^{\circ} \mathrm{F}$. for a few minutes, the paste becomes clear and limpid. The first stage in the digestion is now complete, with the 
formation of soluble starch. If the action of saliva be continued, a number of substances intermediate between starch and sugar are formed, to which the name dextrin has been given. Among these may be mentioned:

a. Evythrodextrin, which gives the reddish-brown color with iodin. As the digestion continues and sugar is formed, the erythrodextrin disappears, giving way to-

b. Achroödextrin, which yields no coloration with iodin, but which may be precipitated by alcohol.

The sugar formed by the action of saliva is maltose, the formula for which is $\mathrm{C}_{12} \mathrm{H}_{22} \mathrm{O}_{11}$. A small quantity of dextrose is also formed.

\section{NERVOUS CIRCULAT ON OF INSALIVATION.}

Afferent or Excitor Nerves.

I. Lingual branch of $5^{\text {th }}$ pair.

2. Glossopharyngeal.
Efferent or Secretory Nerves.

I. Auriculotemporal branch of 5 th pair, for parotid gland.

2. Chorda tympani, for submaxillary and sublingual glands.

The centers regulating the secretion are two-viz., the medulla oblongata and the submaxillary ganglion of the sympathetic, the latter acting antagonistically to the former. Impressions excited by the food in the mouth reach the medulla oblongata through the afferent nerves; motor impulses are there generated which pass outward through the efferent nerves.

Stimulation of the auriculotemporal branch increases the flow of saliva from the parotid gland; division arrests it.

Stimulation of the chorda tympani is followed by a dilatation of the blood-vessels of the submaxillary gland, increased flow of blood (thus acting as a vaso-dilator nerve), and an abundant discharge of a thin saliva ; division of the nerve arrests the secretion.

Stimulation of the cervical sympathetic is followed by a contraction of the blood-vessels, diminishing the flow of blood (thus acting as a vaso-constrictor nerve), and a diminution of the secretion, which now becomes thick and viscid; division of the sympathetic does not, however, completely dilate the vessels. There is evidence of the existence of a local vasomotor mechanism, which is inhibited by the chorda tympani, exalted by the sympathetic.

\section{DEGLUTITION.}

Deglutition is the act of transferring food from the mouth into the stomach, and may be divided into three stages:

I. The passage of the bolus from the mouth into the pharynx. 
2. From the pharynx into the esophagus.

3. From the esophagus into the stomach.

In the first stage, which is entirely voluntary, the mouth is closed and respiration momentarily suspended ; the tongue, placed against the roof of the mouth, arches upward and backward, and forces the bolus in tothe fauces.

In the second stage, which is entirely reflex, the palate is made tense and directed upward and backward by the levatores palati and tensores palati muscles; the bolus is grasped by the superior constrictor muscle of the pharynx and rapidly forced into the esophagus.

The food is prevented from entering the posterior nares by the uvula and the closure of the posterior half-arches (the palatopharyngeal muscles); from entering the larynx by its ascent under the base of the tongue and the action of the epiglottis.

In the third stage the longitudinal and circular muscle-fibers, contracting from above downward, strip the bolus into the stomach. (For Nervous Mechanism of Deglutition, see Medulla Oblongata.)

\section{GASTRIC DIGESTION.}

The Stomach.-Immediately beyond the termination of the esophagus the alimentary canal expands and forms a receptacle for the temporary retention of the food. To this dilatation the term stomach has been applied. This organ is somewhat pyriform in outline, and occupies the upper part of the abdominal cavity. It is about $\mathbf{I} 3$ inches long, 5 deep, and $31 / 2$ wide, and has a capacity of about five pints. It presents two orifices, the cardiac or esophageal, and the pyloric; two curvatures, the lesser and the greater.

The left or cardiac end of the stomach is enlarged, and forms the fundus : the right end is much narrower, and forms the pylorus. The stomach possesses three coats :

I. The serous, or reflection of the peritoneum.

2. The muscular, the fibers of which are arranged in a longitudinal, a circular, and an oblique direction. At the pyloric end the circular fibers increase in number and form a thick ring or band, which is known as the sphincter of the pylorus.

3. The mucous, which is somewhat larger than the muscular coat, and in consequence is thrown into folds or rugre. The surface of the mucous coat is covered by tall, narrow, columnar epithelium.

Gastric Juice.-During the period of time the food remains in the stomach it is subjected to the disintegrating action of an acid fluid, the 
gastric juice. This fluid, secreted from glands in the mucous membrane, is thoroughly incorporated with the food in consequence of the contractions of the muscular coat. The food is gradually liquefied and reduced to a form which partly fits it for passage into the small intestine and for absorp tion into the blood. Gastric juice, when obtained in a pure state, is a clear, colorless fluid, decidedly acid in reaction, with a specific gravity of 1005. It is composed of the following ingredients :

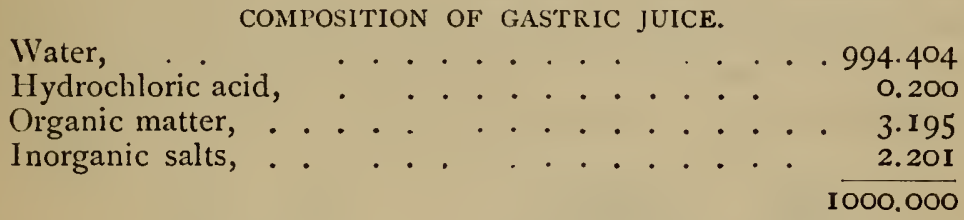

The water forms by far the largest part of this fluid, and serves the purpose of holding the other ingredients in solution, and by its saturating power brings them into relation with the constituents of the food. Of the inorganic salts the sodium and potassium chlorids are the most abundant and important.

The hydrochloric acid, which exists in a free state, is present in variable amounts. In the foregoing table the number of part a thousand is much smaller than is usually stated. According to most observers, hyärochloric acid is present to the extent of from 0.2 to 0.3 part a hundred. Thougl secreted as soon as the food enters the stomach, the acid can not be detected in the free state until after the lapse of from thirty to forty minutes. It acidulates the food and prevents fermentative changes.

The pepsin, which is present in gastric juice associated with the organic matter, is a hydrolytic ferment or enzyme. When freed from its associations and obtained in a pure state, pepsin presents the characteristics of a colloid body, and resembles in its reactions the albuminoids. It has the power, when brought into relation with acidulated proteids, of transforming them into new forms capable of absorption into the blood.

Rennin.-In addition to pepsin a second ferment exists in the gastric juice, to which the term rennin has been given. It possesses the power of coagulating the caseinogen of milk. It exists in the mucous membrane, from which it can be extracted by appropriate means. When rennin acts on caseinogen, the latter is split into insoluble casein and a soluble albumin. Calcium phosphate is essential to the action of this enzyme.

Gastric Glands.-Embedded within the mucous membrane are to be found enormous numbers of tubular glands, which, though resembling one 
another in general form, differ in their histologic details in various portions of the stomach.

In the cardiac end or fundus the glands consist of several long tubules, opening into a short, common duct, which opens by a wide mouth on the surface of the unucous membrane. Each gland consists primarily of a basement membrane lined by epithelial cells. In the duct the epithelium is of the columnar variety, resembling that covering the surface of the mucous membrane. The secretory portion of the tubule is lined by a layer of short, polyhedral, granular, and nucleated cells, which, as they border the lumen of the tubule, and thus occupy the central portion of the gland, are termed central cells. At irregular intervals, between the central cells
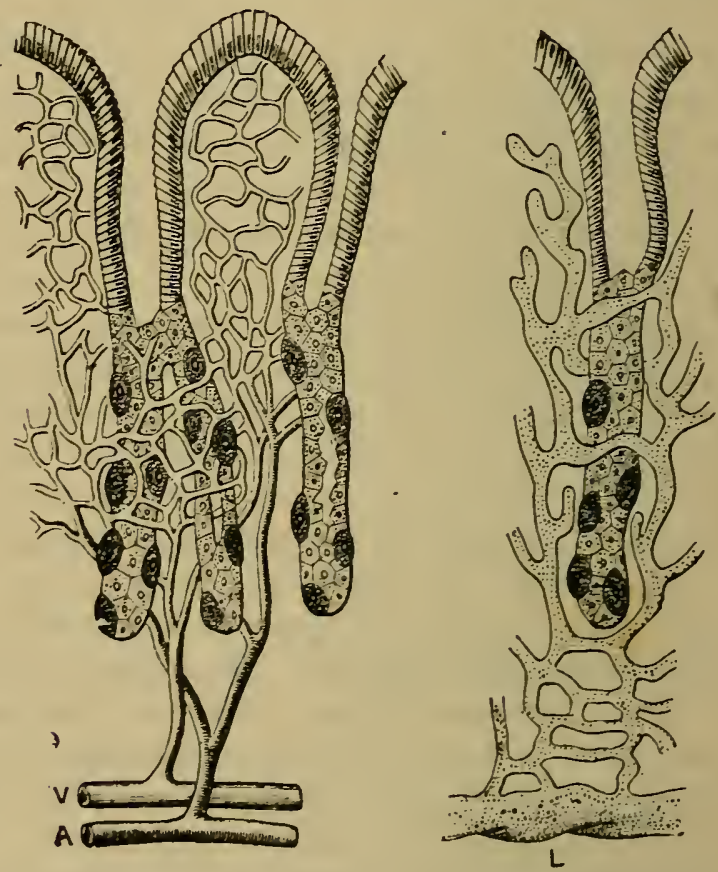

FIG. 13.

Diagram showing the relation of the ultimate twigs of the blood-vessels, $\mathrm{V}$ and $\mathrm{A}$, and of the absorbent radicles to the glands of the stomach and the different kinds of - epithelium-viz., above cylindric cells; small, pale cel $s$ in the lumen, outside which are the dark ovoid cells.-(Yeo's" Text-book of I'liysiology.")

and the wall of the tubule, are found large, oval, reticulated cells, which, on account of their position, are termed parietal cells. (See Fig. 13.)

Each parietal cell is in relation with a system of fine canals, which open directly into the lumen of the gland. It is estimated that the fundus con- 
tains about five million glands. In the pyloric end of the stomach the glands are generally branched at their lower extremities, and the common duct is long and wide. The duct is lined by columnar epithelium, while the secreting part is lined by short, slightly columnar, granular cells. The parietal cells are entirely wanting. The epithelium covering the surface of the nucous membrane is tall, narrow and cylindric in shape, and consists of mucus-secreting goblet cells. The outer half of the cell contains a substance, mucinogen, which produces mucin. The gastric glands in both situations are surrounded by a fine connective tissue, which supports blood-vessels, nerves, and lymphatics.

Changes in the Cells During Secretion.-During the periods of rest and secretory activity the cells of the glands undergo changes in structure which are supposed to be connected with the production of the pepsin and liydrochloric acid. During rest, the protoplasm of the central cells becomes filled with granular matter; during the time of secretion this disappears, presumably passing into the lumen of the tubule, and as a result the protoplasm becomes clear and hyaline in appearance. The granular material is probably the mother substance, pepsinogen, which, inactive in itself, yields the active ferment, pepsin. The parietal cells during digestion increase in size, but do not become granular. It is at this period that they secrete the hydrochloric acid. After digestion they rapidly diminish in size and return to their former condition. The pyloric glands secrete pepsin only.

Mechanism of Secretion.-In the intervals of digestion the mucous membrane of the stomach is covered with a layer of mucus. As soon as the food passes from the esophagus into the stomach, the blood-vessels dilate, the circulation becomes more active, and the membrane assumes a bright red appearance. Coincidentally, small drops of gastric juice begin to exude from the glands, which, as they increase in number, run together and trickle down the sides of the stomach. This pouring out of fluid continues during the presence of food in the stomach.

The secretion of gastric juice is a reflex act, taking place through the central nervous systen and called forth in response to the stimulus of food in the stomach. That the central nervous system also directly influences the production of the secretion is shown by the fact that emotion, such as fear or anger, will arrest or vitiate the normal secretion. The reflex nature of the process can be shown by experimentation upon the pneumogastric nerve. If during digestion, when the peristaltic movements are active and the gastric mucous membrane is flushed and covered with gas- 
tric juice, the pneumogastric nerves are divided on both sides, the mucous membrane becomes pale, the secretion is arrested, and the peristaltic movements become less marked. Stimulation of the peripheral end produces no constant effects; stimulation of the central end, however, is at once followed by dilatation of the vessels, flushing of the mucous membrane, and reëstablishment of the secretion. It is evident, therefore, that during digestion afferent impulses are passing up the pneumogastrics to the medulla; efferent impulses, in all probability, pass through the fibers of the sympathetic nervous system to the blood-vessels and glands concerned in the elaboration of the gastric juice. After all the nerve connections of the stomach are divided, the secretion of a small quantity of juice continues for several days. This has been attributed to the action of a local nervous mechanism and to the direct action of the food upon the protoplasm of the secreting cells.

Chemic Action of the Gastric Juice.-By the combined influence of the contraction of the muscular walls, the action of the gastric juice, and the temperature, the food is reduced to a semiliquid condition and acquires a distinct acid odor. This semifluid mass will vary in composition and appearance according to the nature of the food. To this matter the term chyme has been given.

Meat is rapidly disintegrated by the solution of-its connective tissue. The fibers thus separated are readily broken up into particles by solution of the sarcolemma. Well-cooked meat is more easily digested, owing to the conversion of the connective tissue into gelatin.

Connective tissues in the raw or imperfectly gelatinized condition are very slowly dissolved. Cartilage, tendons, and even bones will in time be corroded and liquefied.

Vegetables are not easily digested unless thoroughly prepared by sufficient cooking. The nutritive principles are inclosed by cellulose walls, which are not affected by gastric juice. The influence of heat and moisture softens and ruptures the cellulose walls so as to permit the introduction of gastric juice and the solution of its nutritive principles.

The principal action of the gastric juice, however, is the transformation of the different proteid principles of the food into peptones, the intermediate stages of which are due to the influence of the acid and pepsin respectively. As soon as any one of the albumins is penetrated by the acid it is converted into acid-albumin, a fact which indicates that the first step in gastric digestion is the acidification of the proteids. This having been accomplished, the pepsin becomes operative and in a varying length of 
time transforms the acid-albumin into a new form of proteid termed peptone, of which, as indicated by chemic tests, there are probably two forms, hemi-and anti-peptone. In this transformation it is possible to isolate intermediate bodies by the addition of ammonium sulphate, to which the term alhumose or proteose has been given. Inasmuch as two forms of peptone can be isolated after complete digestion of any given proteid, it is assumed, from this and other facts, that their appearance has been preceded by two forms of albumose, hemi- and anti-. This supposed change is represented in the following scheme :

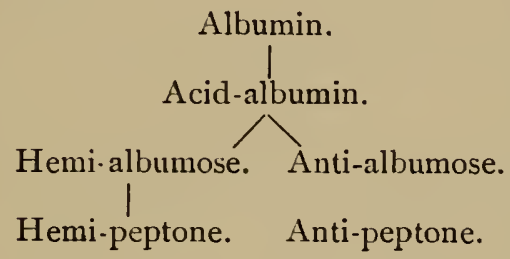

From the fact that one form of peptone-hemi-under the influence of the pancreatic ferment trypsin can be decomposed into leucin, tyrosin, aspartic acid, etc., it is believed that all the simple proteids contain two distinct groups or radicles termed hemi-and anti-radicles, and that it this fact that determines the line of cleavage and the characteristics of the cleavage products.

Peptones.-Peptones are the final products of the digestion of proteid bodies, and differ from the bodies from which they are derived in the following particulars :

I. They are diffusible, -i. e., capable of passing readily through animal membranes, - a condition essential for their absorption.

2. They are soluble in water and in saline solution.

3. They are non-coagulable by heat and nitric or acetic acids. They can be readily precipitated, however, by tannic acid, by bile acids, and by mercuric chlorid.

4. They are absorbable and assimilab'e, soon becoming transformed into serum-albumin.

The duration of gastric digestion will depend largely upon the quantity and quality of the food. The digestion of the average meal occupies from three to five hours.

Movements of the Stomach.-As soon as digestion commences the cardiac and pyloric orifices are closed; the walls of the stomach contract upon the food, and a peristaltic action begins, which carries the food along the greater and lesser curvatures, and thoroughly incorporates it with the 
gastric juice. As soon as any portion of the food is digested, it passes through the pylorus into the intestine.

TABLE SHOWING DIGESTIBILITY OF VARIOUS ARTICLES OF FOOD.

Eggs, whipped, ............. I

Hours. Minutes.

" soft-boiled, . . . . . . . . . . 3

" hard-boiled, . . . . . . . . . . 3

Oysters raw, . . . . . . . . . . 2

"s stewed,

Lamb, broiled,

Veal,

. . . . . . . . . . 2

Pork, roasted, . . . . . . . . . . . 5

Beefsteak, broiled, . . . . . . . . 3

Turkey, roasted, ............ . . 2

('hicken, boiled

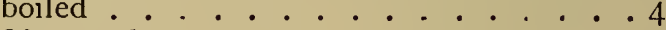

" fricasseed, . . . . . . . . . . . 2

Duck, roasted .

Soup, barley, boiled, . . . . . . . . . I

" bean, " . . . . . . . . . . 3

" chicken, " . . . . . . . . . . 3

" mutton, " . . . . . . . . . . . . 3

Iiver, beef, broiled, . . . . . . . . . 2

Sausage, " . . . . . . . . . . . 3

Green corn, boiled . . . . . . . . . . 34

Beans, ". . . . . . . . . . 2

Potatoes, roasted . . . . . . . . . . 2

" boiled,.............. . . 3 30

Cabbage, " . . . . . . . . . . . . 4

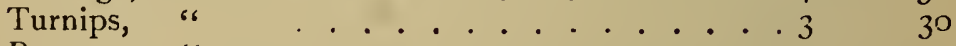

Beets, "

Parsnips, " . . . . . . . . . . . . 2 30

\section{INTESTLNAL DIGESTION.}

The process of digestion as it takes place in the small intestine is exceedingly important and complex, and is brought about by the action of the pancreatic juice, the bile, and the intestinal juice.

The contents of the stomach at the close of gastric digestion consist of water, inorganic salts, peptones, undigested albumins and starches, maltose, cane-sugar, liquefied fats, cellulose, and the indigestible portions of meats, cereals, fruits, etc. This so-called chyme is quite acid in reaction, and upon its passage through the now open pylorus into the intestine it excites a reflex stimulation and secretion of the intestinal fluids, which are decidedly alkaline in reaction, and which have a neutralizing action on the chyme. As soon as the latter becomes alkaline and gastric digestion is arrested, the 
various phases of intestinal digestion begin which eventuate in the transformation of all the remaining undigested nutritive materials into absorbable and assimilable compounds.

The small intestine is about 22 feet in length and about $1 \frac{1}{2}$ inches in diameter. Like the stomach, it possesses three coats, as follows :

I. The serous, or peritoneal.

2. The muscular, the fibers of which are arranged for the most part circularly. Some of the fibers are so arranged as to form longitudinal bands.

3. The mucous, which preserts a series of transverse folds, known as the valvula conniventes.

Intestinal Glands.- In that portion of the small intestine known as the duodenum are to be found a number of small, branched, tubular glands (Brunner's), the acini of which are lined by short, cylindric cells, similar to those lining the pyloric glands. From the duodenum to the termination of the intestine the mucous membrane contains an enormous number of tubular glands (Lieberkühn's), formed by an inversion of the basement membrane and lined by epithelial cells. The common secretion of these intestinal glands forms the intestinal juice. This is a thin, opalescent, slightly yellowish fluid, alkaline in reaction, and contains water, salts, and proteid matter.

The function of the intestinal juice is but incompletely known. It appears to have the power of converting starch into dextrose; it is doubtful whether it is capable of digesting either albumins or fats. Its most distinctive action is the inversion of cane-sugar, maltose, and lactose into dextrose, thus preparing them for absorption. This change is dependent on the presence of a ferment body known as invertin.

The pancreatic juice is secreted by the pancreas, a flattened gland, about six inches long, running transversely across the posterior wall of the abdomen behind the stomach; its duct opens into the duodenum.

The pancreas is similar in structure to the salivary glands, and consists of a system of ducts terminating in acini. The acini are tubular or flaskshaped, and consist of a basement membrane lined by a layer of cylindric, conic cells, which encroach upon the lumen of the acini. The cells exhibit a difference in their structure (Fig. 14), and may be said to consist of two zones-viz., an outer parietal zone, which is transparent and apparently homogeneous, staining rapidly with carmin; an inner zone, which ${ }^{\circ}$ borders the lumen, and is distinctly granular and stains but slightly with carmin. These cells undergo changes similar to those exhibited by the 
cells of the salivary glands during and after active secretion. As soon as the secretory activity of the pancreas is established, the granules disappear, and the inner granular layer becomes reduced to a very narrow border, while the outer zone increases in size and occupies nearly the entire cell. During the intervals of secretion, however, the granular layer reappears and increases in size until the outer zone is reduced to a minimum. It would seem that the granular matter is formed by the nutritive processes occurring in the gland during rest, and is discharged during secretory activity into the ducts, and takes part in the formation of the pancreatic secretion.

The pancreatic juice is transparent, colorless, strongly alkaline, and viscid, and has a specific gravity of I040. It is one of the most im-
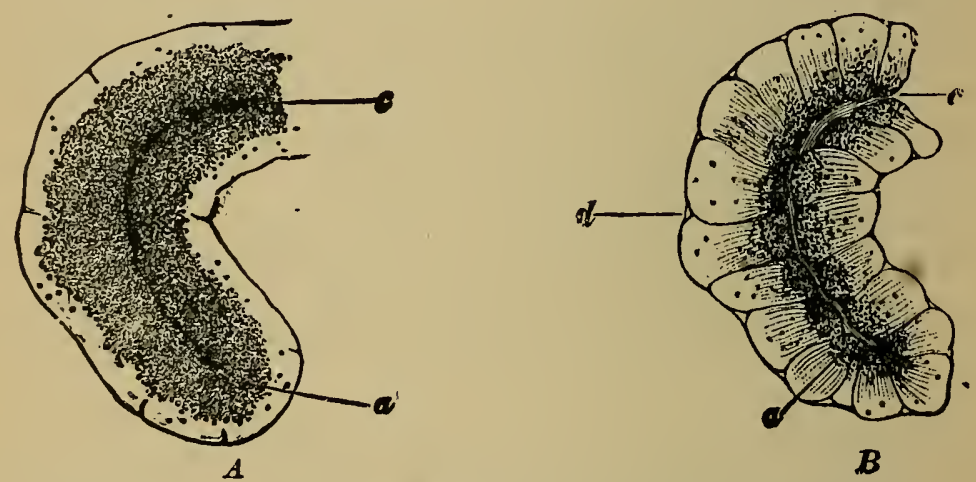

Fig. i4.-One Saccule of the Pancreas of the Rabeit in Different States of Activity.-(Yeo's "Text-book of Physiology," after Kühne and Lea.)

$A$. After a period of rest, in which case the outlines of the cells are indistinct and the

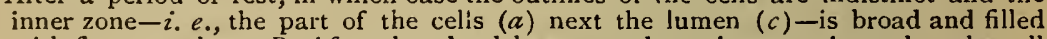
with fine granules. $B$. After the gland has poured out its secretion, when the cell outlines $(d)$ are clearer, the granular zone $(a)$ is smaller, and the clear outer zone is wider.

portant of the digestive fluids, as it exerts a transforming influence upon all classes of alimentary principles, and has been shown to contain at least three distinct ferments. It has the following composition :

COMIOSITION OF PANCREATIC JUICE.

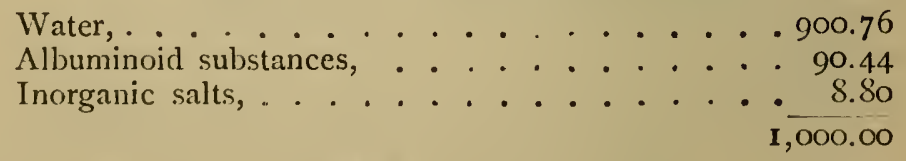

The pancreatic juice is characterized by its action :

I. Upon starch. When starch is subjected to the action of the juice, it is at once transformed into maltose; the change takes place more rapidly 
than when saliva is added. This action is caused by the presence of a special ferment, amylopsin.

2. Upon albumin. The proteid bodies which escape digestion in the stomach are converted into peptones by the action of the alkali and ferment. The first effect of the alkali is to change the proteid into an alkali-albumin, a fact which indicates that in the digestion of albumin by pancreatic juice, the first stage is alkalinization. This having been accomplished, the ferment trypsin transforms the alkali-albumin into peptone, of which, as in gastric digestion, there are two forms, hemi-and anti-peptone. For the same reason it is believed that here also these bodies are preceded in their development by albumoses, of which there are probably two forms. Long-continued action of the pancreatic juice as previously stated, decomposes the hemi-peptone into leucin, tyrosin, etc.

3. Upon fats. The most striking action of the pancreatic juice is the emulsification of the fats or their subdivision into minute particles of microscopic size. This change takes place rapidly, and depends upon the alkalinity of the fluid and the quantity of albumin present, combined with the intestinal movements. The neutral fats are also decomposed into their corresponding fatty acids and glycerin; the acids thus set free unite with the alkaline bases present in the intestine and form soaps. This decomposition of the neutral fats is caused by the ferment, steapsin.

The bile has an important function in the elaboration of the food and in its preparation for absorption. It is a golden-brown, viscid fluid, having a neutral or alkaline reaction and a specific gravity of 1020 .

COMPOSITION OF BILE.

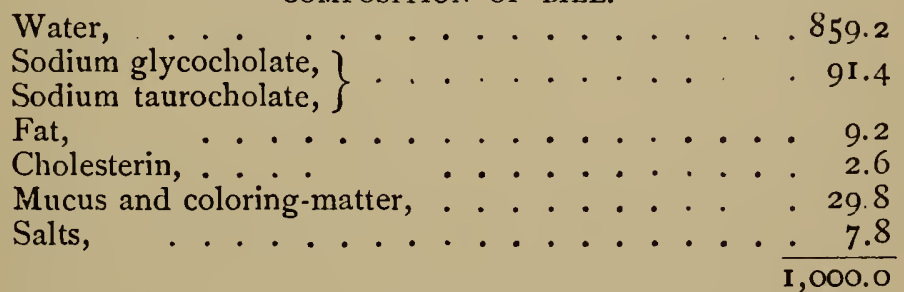

The biliary salts; sodium glycocholate and taurocholate, are characteristic ingredients, and by the process of secretion are formed in the liver from materials furnished by the blood. It is probable that they are derived from the nitrogenized compounds, though the stages in the process are unknown. They are reabsorbed from the small intestine to play some ulterior part in nutrition.

Cholesterin is a product of waste taken up by the blood from the nerve tissues and excreted by the liver. It crystallizes in the form of rhombic 
plates, which are quite transparent. When retained within the blood, it gives rise to the condition of cholesteremia, attended with severe nervous symptoms. It is given off in the feces under the form of stercorin.

The coloring-matters which give the tints to the bile are biliverdin and bilirubin, and are probably derived from the coloring-matter of the blood. Their presence in any fluid can be recognized by adding to it nitric acid containing nitrous acid, when a play of colors is observed, beginning with green, blue, violet, red, and yellow.

The bile is both a secretion and an excretion; it is constantly being formed and discharged by the hepatic ducts into the gall-bladder, in which it is stored up during the intervals of digestion. As soon as food enters the intestines it is poured out abundantly by the contraction of the walls of the gall-bladder.

The amount secreted in twenty-four hours is about $2 \frac{1}{2}$ pounds.

\section{Functions of the Bile:}

I. It assists in the emulsification of the fats and promotes their absorption.

2. It tends to prevent putrefactive changes in the food.

3. It stimulates the secretion of the intestinal glands, and excites the normal peristaltic movement of the bowels.

The digested food, the chyme, is a grayish, pultaceous mass, but as it passes through the intestines it becomes yellow from admixture with the bile. It is propelled onward by vermicular motion - by the contraction of the circular and longitudinal muscle-fibers.

During the passage of the digesting food through the intestinal canal the nutritive products - the peptones, the dextrose and levulose, the fatty emulsions, the fatty acids and their soaps - are absorbed into the blood, while the undigested residue is carried onward by the peristaltic movements through the ileo-cecal valve into the large intestine.

Intestinal Fermentation. - Owing to the favorable conditions for fermentative and putrefactive processes- - .g., heat, moisture, oxygen, microorganisms - the food, when consumed in excessive quantity or when acted upen by defective secretions, undergoes a series of decomposition changes which are attended by the production of gases and various chemic compounds. Grape-sugar and maltose are partially split into lactic acid, this into butyric acid, carbon dioxid, and hydrogen. Fats are reduced to glycerol and fatty acids; the glycerol, according to the organisms present, yields succinic and other fatty acids, carbon dioxid, and hydrogen.

The proteids, under the prolonged action of the pancreatic juice, are decomposed, and yield leucin and tyrosin; the former is split into valerianic 
acid, ammonia, and carbon dioxid; the latter is split into indol, which is the antecedent of indican in the urine. Skatol is another proteid derivative constantly present in the fecal substance.

The large intestine extends from the ileo-cecal valve to the anus, and is about five feet in length. Like the stomach it consists of three coats : the serous, the inuscular, and mucous. The mucous membrane contains a number of mucous glands, the secretion from which lubricates the surface of the canal. The ascending portion of the large intestine possesses the power of absorption, and hence its contents become less liquid and more consistent. As the residne passes toward the sigmoid flexure it acquires the characteristics of fecal matter. This residue consists of the undigested portions of the food, decomposition products, mucus, and inorganic salts.

Defecation is the voluntary act of extruding the feces from the rectum, and is accomplished by a relaxation of the sphincter ani muscle and by the contraction of the muscular walls of the rectum, aided by the contraction of the abdominal muscles.

\section{ABSORPTION.}

The term absorption is applied to the passage or transference of material into the blood from the tissues, from the serous cavities, and from the mucous surfaces of the body. The most important of these surfaces, especially in its relation to the formation of the blood, is the mucous surface of the alimentary canal; for it is from this organ that new materials are derived which maintain the quality and quantity of the blood. The absorption of materials from the interstices of the tissues is to be regarded rather as a return to the blood of liquid nutritive material which has escaped from the blood-vessels for nutritive purposes, and which, if not returned, would lead to an accumulation of such fluid and the development of dropsical conditions.

The anatomic mechanisms involved in the absorptive process are, primarily, the lymph-spaces, the lymph-capillaries, and the blood-capillaries; secondarily, the lymphatic vessels and larger blood-vessels.

Lymph-spaces, Lymph-capillaries, Blood-capillaries.-Everywhere throughout the body, in the intervals between connective-tissue bundles and in the interstices of the several structures of which an organ is composed, are found spaces of irregular shape and size, determined largely by the nature of the organ in which they are found, which have been termed 
lymph-spaces or lacunce, from the fact that during the living condition they are continually receiving the lymph which has escaped from the bloodvessels throughout the body. In addition to the connective-tissue lymphspaces, various observers have described special lymph-spaces in the testicle, kidney, liver, thymus gland, and spleen; in all secreting glands between the basement membrane and blood-vessels; around blood-vessels (perivascular spaces), and around nerves. The serous cavities of the body - peritoneal, pleural, pericardial, etc.-may also be regarded as lymphspaces, which are in direct communication by open mouths or stomata with the lymphatic capillaries. This method of communication is not only true of serous membranes, but to some extent also of mucous membranes. The cylindric sheaths and endothelial cells surrounding the brain, spinal cord, and nerves can also be looked upon as lymph-spaces in connection with lymph-capillaries.

The lymphatic capillarics, in which the lymphatic vessels proper take their origin, are arranged in the form of plexuses of quite irregular shape. In most situations they are intimately interwoven with the blood-vessels, from which, however, they can be readily distinguished by their larger caliber and irregular expansions. The wall of the lymph-capillary is formed by a single layer of epithelioid cells, with sinuous outlines, and which accurately dovetail with one another. In no instance are valves found. In the villus of the small intestine the beginning of the lymphatic is to be regarded as a lymph-capillary, generally club-shaped, which at the base of the villus enters a true lymphatic; at this point a valve is situated, which prevents regurgitation. The lymphatic capillaries anastomose freely with one another, and communicate on the one hand with the lymph-spaces, and on the other with the lymphatic vessels proper.

As the shape, size, etc., of both lymph-spaces and capillaries are determined largely by the nature of the tissues in which they are contained, it is not always possible to separate the one from the other. Their function, however, may be regarded as similar-viz., the collection of the lymph which has escaped from the blood-vessels, and its transmission onward into the regular lymphatic vessels.

The blood-capillaries not only permit the escape of the liquid nutritive portions of the blood through their delicate walls, but are also engaged in the reabsorption of this transudate, as well as in the absorption of new materials from the alimentary canal. The extensive capillary network which is formed by the ultimate subdivision of the arterioles in the submucous tissue and villi of the small intestine forms an anatomic arrangement well adapted for absorption. It is now well known that in the 
absorption of the products of digestion the blood-capillaries are more active than the lymphatic capillaries.

Lymphatic Vessels. - These constitute a system of minute, delicate transparent vessels, found in nearly all the organs and tissues of the body. Having their origin at the periphery in the lymphatic capillaries and spaces, they rapidly converge toward the trunk of the body and empty into the thoracic duct. In their course they pass through numerous small ovoid bodies, the lymphatic glands.

The lymphatic vessels of the small intestines-the lacteals-arise within the villous processes which project from the inner surface of the intestine throughout its entire extent. The wall of the villus is formed by an elevation of the basement membrane, and is covered by a layer of columnar epithelial cells. The basis of the villus consists of adenoid tissue, a fine plexus of blood-vessels, unstriped muscle-fibers, and the lacteal vessel. The adenoid tissue consists of a number of intercommunicating spaces, containing leukocytes. The lacteal vessel possesses a thin but distinct wall composed of endothelial plates, with here and there openings which bring the interior of the villus into communication with the spaces of the adenoid tissue.

The structure of the larger vessels resembles that of the veins, consisting of three coats :

I. External, composed of fibrous tissue and muscle-fibers, arranged longitudinally.

2. Middle, consisting of white fibers and yellow elastic tissue, non-striated muscle-fibers, arranged transversely.

3. Internal, composed of an elastic membrane, lined by endothelial cells. Throughout their course are found numerous semilunar valves, opening toward the larger vessels, formed by a folding of the inner coat and strengthened by connective tissue.

Lymphatic Glands. - The lymphatic glands consist of an external capsule composed of fibrous tissue which contains non-striped musclefibers ; from its inner surface septá of fibrous tissue pass inward and subdivide the gland-substance into a series of compartments, which communicate with one another. The blood-vessels which penetrate the gland are surrounded by fine threads, forming a follicular arrangement, the meshes of which contain numerous lymph-corpuscles. Between the follicular threads and the wall of the gland lies a lymph-channel traversed by a reticulum of adenoid tissue. The lymphatic vessels, after penetrating this capsule, pour their lymph into this channel, through which it passes ; it is then collected 


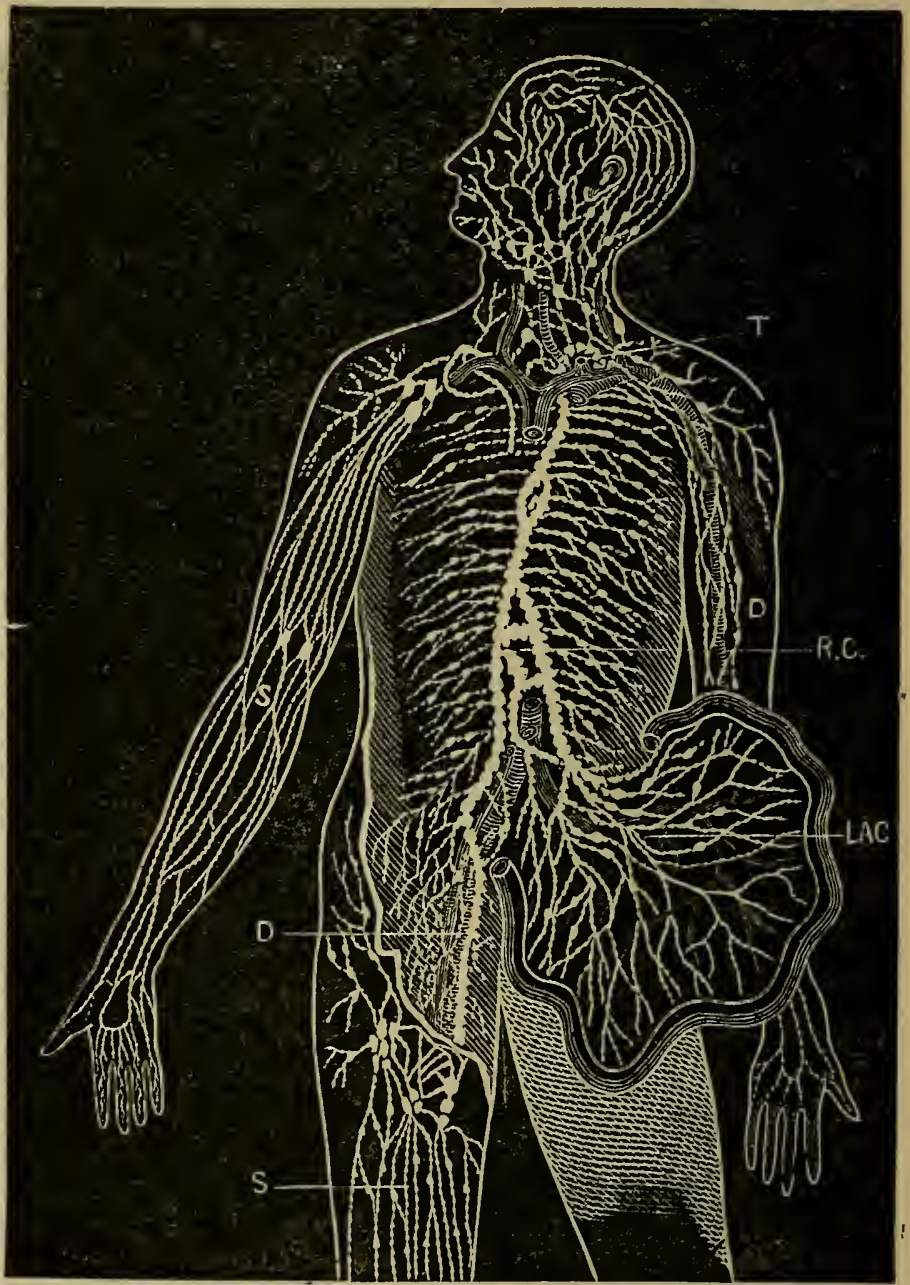

Fig. 15.-Diagram Showing the Course of the Main Thunks of the Absorbent System.-(Yeo's "Text-Book of Physiology.")

The lymphatics of lower extremities (D) meet the lacteals of intestines (LAC) at the receptaculum chyli ( $R C$ ), where the thoracic duct begins. The superficial vessels are shown in the diagram on the right arm and leg (S), and the deeper ones on the arm to the left (D). The glands are here and there shown in groups. 'The small right duct opens into the veins on the right side. The thoracic duct opens into the union of the great veins of the left side of the neck ( $T$ ). 
by the efferent vessels and transmitted onward. The lympin-corpuscles which are washed out of the gland into the lymph-stream are formed, most probably, by division of preëxisting cells.

The thoracic duct is the general trunk of the lymphatic system; into it the vessels of the lower extrcmities, of the abdominal organs, of the left side of the head, and of the left arm empty their contents. It is about twenty inches in length, arises in the abdomen, opposite the third lumbar vertebra, by a dilatation (the receptaculum chyli), ascends along the vertebral column to the seventh cervical vertebra, and terminates in the venous system at the junction of the internal jugular and subclavian veins on the left side. The lymphatics of the right side of the head, of the right arm, and of the right side of the thorax terminate in the right thoracic duct, about one inch in length, which joins the venous system at the junction of the internal jugular and subclavian on the right side.

The general arrangement of the Iymphatic vessels is show in figure 15 .

The blood-vessels which are concerned in the conduction of fresh nutritive material from the alimentary canal have their origin in the elaborate capillary network in the mucous membrane. The small veins which emerge from the network gradually unite, forming larger and larger trunks, which are known as the gastric, superior, and inferior mesenteric veins. These finally unite to form the portal vein, a short trunk about three inches in length. The portal vein enters the liver at the transverse fissure, after which it forms a fine capillary plexus ramifying throughout the substance of the liver; from this plexus the hepatic veins take their origin, and finally empty the blood into the vena cava inferior. (See Fig. I6.)

Absorption of Food.-Physiological experiments have demonstrated that the agents concerned in the absorption of new materials from the alimentary canal are :

I. The blood-vessels of the entire canal, but more particularly those uniting to form the portal vein.

2. The lymphatics coming from the small intestine, which converge to empty into the thoracic duct.

As a result of the action of the digestive fluids upon the different classes of food principles-albumins, sugars, starches, and fats - there are formed peptones, glucose, and fatty emulsion, which differ from the former in being highly diffusible-a condition essential to their absorption. In order that these substances may get into the blood, they must pass through the layer of cylindric epithelial cells and the underlying basement membrane, and into the lymph-spaces of the villi and submucous tissue. The mechanism 
by which the cells effect this passage of the food is but imperfectly understood. Osmosis and filtration are conditions, however, made use of by the cells in the absorptive process.

The products of digestion find their way into the general circulation by two routes:

1. The water, peptones, slucose, and soluble salts, after passing into the

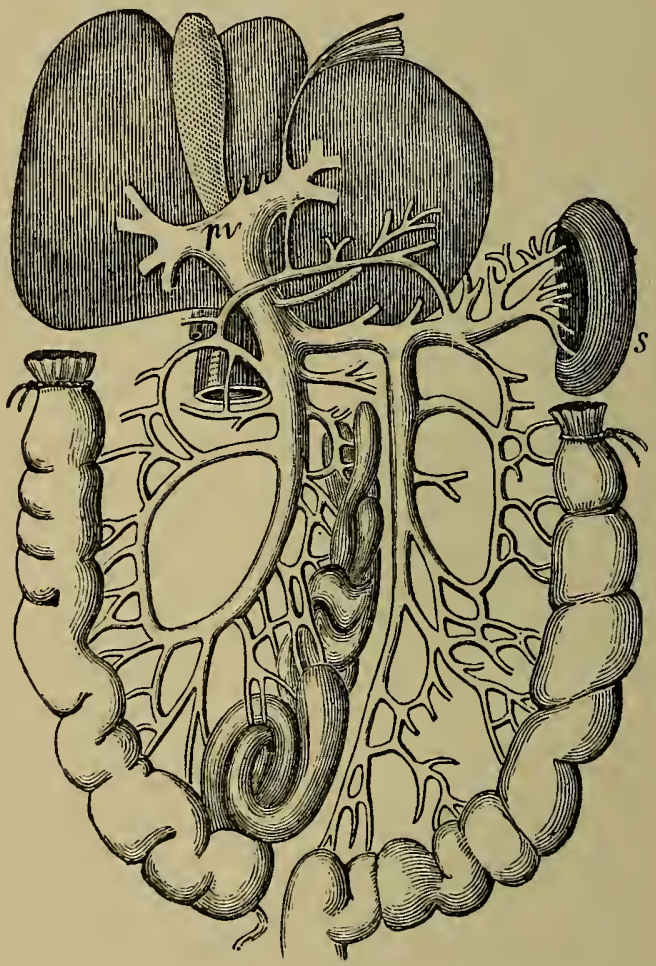

FIG. I6.

Diagram of the portal vein $(p v)$ arising in the alimentary tract and spleen $(s)$, and carrying the blood from these organs to the liver.-(Yeo's "Text-book of Physiology.")

lymph-spaces of the villi, pass through the wall of the capillary bloodvessel; entering the blood, they are carried to the liver by the vessels uniting to form the portal vein; emerging from the liver, they are empted into the inferior vena cava by the hepatic vein.

2. The emulsified fat enters the lymph-capillary in the interior of the villus; by the contraction of the layer of muscle-fibers surrounding 
it its contents are forced onward into the lymphatic vessels or lacteal, thence into the thoracic duct, and finally into the circulation at the junction of the internal jugular and subclavian veins on the left side.

Absorption of Lymph.- Similar to the absorption of food from the alimentary canal is the absorption of lymph from the lymph-spaces of the organs and tissues. During the passage of the blood through the capillary blood-vessels a portion of the liquor sanguinis, or plasma, or lymph, passes through the capillary wall out into the lymph-spaces. The tissue-cells are thus bathed with this new materal ; from it those substances are selected which are necessary for their growth, repair, and all purposes of nutrition. An excess of nutritive material, far beyond the needs of the tissues, transudes from the blood-vessels, and it is this excess which is absorbed by the lymphatics and returned to the blood by the thoracic duct. It is quite probable, also, that a portion of this transudate is reabsorbed by the blood-vessels.

Properties and Composition of Lymph and Chyle.-Lymph, as found in the lymphatic vessels of animals, is a clear, colorless, or opalescent fluid, having an alkaline reaction, a saline taste, and a specific gravity of about 1040. It holds in suspension a number of corpuscles resembling in their general appearance the white corpuscles of the blood. Their number has been estimated at 8,200 per cubic millimeter, though the number varies in different portions of the lymphatic system. As the lymph flows through the lymphatic gland it receives a large addition of corpuscles. Lymph-corpuscles are granular in structure, and measure $\frac{1}{2500}$ of an inch in diameter. When withdrawn from the vessels, lymph undergoes a spontaneous coagulation similar to that of the blood, after which it separates in serum and clot.

\section{COMPOSITION OF LYMPII.}

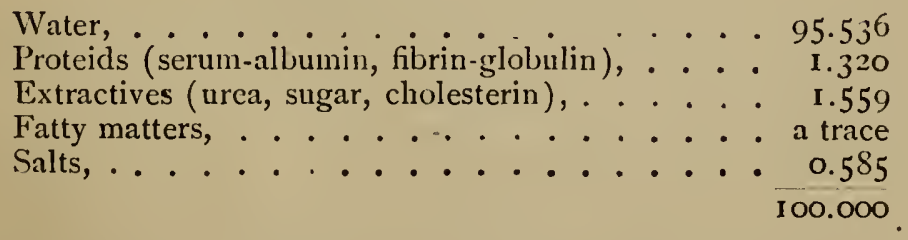

Chyle.-Chyle is the fluid found in the lymphatic vessels, coming from the small intestine after the digestion of a meal containing fat. In the intervals of digestion the fluid of these lymphatics is identical in all respects with the lymph found in all other regions of the body. As soon 
as the emulsified fat passes into the lymphatic vessels and mingles with the lymph it becomes milky white in color, and the vessels which previously were invisible become visible, and resemble white threads running between the layers of the mesentery. Chyle has a composition similar to that of lymph, but it contains, in addition, numerous fatty granules, each surrounded by an albuminous envelope. When examined microscopically, the chyle presents a fine molecular basis, made up of the finely divided granules of fat.

COMPOSITION OF CHYLE.

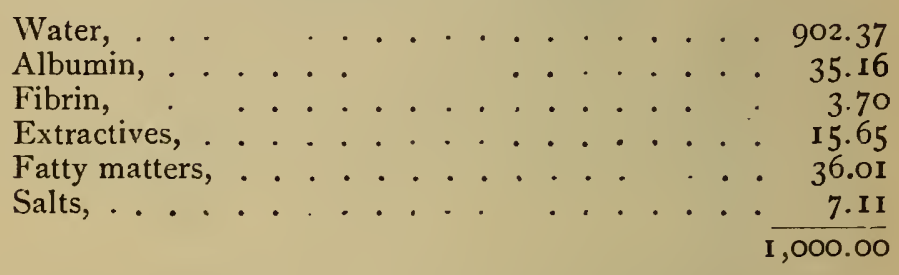

Forces Aiding the Movement of Lymph and Chyle.--The lymph and chyle are continually moving in a progressive manner from the periphery or beginning of the lymphatic system to the final termination of the thoracic duct. The force which primarily determines the movement of the lymph has its origin in the beginnings of the lymphatic vessels, and depends upon the difference in pressure here and the pressure in the thoracic duct. The greater the quantity of fluid poured into the lymph-spaces, the greater will be the pressure and, consequently, the movement. The first movement of chyle is the result of a contraction of the muscle-fibers within the walls of the villus. At the time of contraction the lymphatic capillary is compressed and shortened, and its contents are forced onward into the true lymphatic. When the muscle-fibers relax, regurgitation is prevented by the closure of the valve in the lymphatic at the base of the villus.

As the walls of the lymphatic vessels contain muscle-fibers, when they become distended these fibers contract and assist materially in the onward movement of the fluid.

The contraction of the general muscular masses in all parts of the body, by exerting an intermittent pressure upon the lymphatics, also hastens the current onward; regurgitation is prevented by the closure of valves which everywhere line the interior of the vessels.

The respiratory movements aid the general flow of both lymph and chyle from the thoracic duct into the venous blood. During the time of an inspiratory movement the pressure within the thorax, but outside the lungs, 
undergoes a diminution in proportion to the extent of the movement; as a result, the fluid in the thoracic duct outside of the thorax, being under a higher pressure, flows more rapidly into the venous system. At the time of an expiration, the pressure rises and the flow is temporarily impeded, only to begin again at the next inspiration.

\section{BLOOD.}

The blood is a nutritive fluid containing all the elements necessary for the repair of the tissues; it also contains principles of waste absorbed from the tissues, which are conveyed to the various excretory organs and by them eliminated from the body.

The total a mount of blood in the body is estimated to be about one eighth of the body-weight; from sixteen to eighteen pounds in an individual of average physical development. The quantity varies during the twenty-four hours, the maximum being reached in the afternoon, the minimum in the early morning hours.

Blood is an opaque, red fluid, having an alkaline reaction, a saline taste, and a specific gravity of 1055 .

The opacity is due to the refraction of the rays of light by the elements of which the blood is composed. The color varies in hue, from a bright scarlet in the arteries to a deep purple in the veins, due to the presence of a coloring-matter-hemoglobin-in different degrees of oxidation.

The alkalinity is constant, and depends upon the presence of the alkaline sodium phosphate, $\mathrm{Na}_{2} \mathrm{HPO}_{4}$.

The saline taste is due to the amount of sodium chlorid present.

Within the limits of health the specific gravity ranges from 1045 to 1075 .

The odor of the blood is characteristic, and varies with the animal from which it is drawn; it is due to the presence of caproic acid.

The temperature of the blood ranges from $9 \oint^{\circ} \mathrm{F}$. at the surface to $107^{\circ}$ $F$. in the hepatic rein; it loses heat by radiation and evaporation as it approaches the extremities and as it passes through the lungs.

\section{Blood Consists of Two Portions:}

I. The liquor sansuinis or plasma, a transparent, colorless fluid, in which are floating-

2. Red and white corpuscles, these constituting by weight less than one balf ( 40 per cent.) of the entire amount of blood. 
COMPOSITION OF PLASMA.

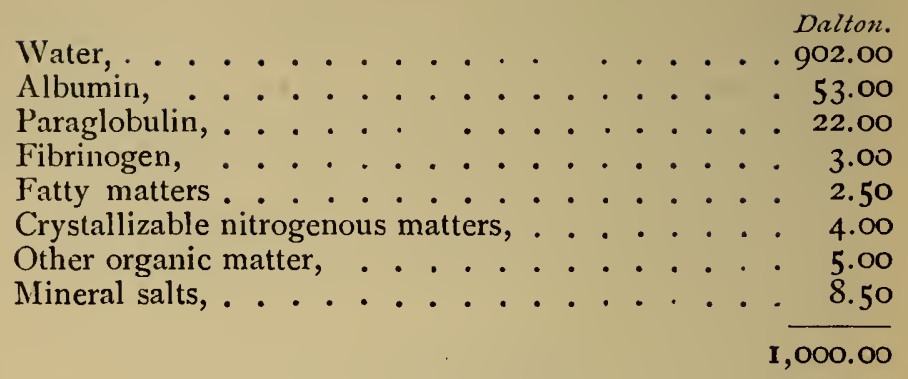

Water acts as a solvent for the inorganic matters and holds in suspension the corpuscular elements.

Albumin is the nutritive principle of the blood; it is absorbed by the tissues to repair their waste and is transformed into the organic basis characteristic of each structure.

Paraglobulin or fibrinoplastin is a soft, amorphous substance precipitated by sodium chlorid in excess, or by passing a strean of carbonic acid through dilute serum.

Fibrinogen also can be obtained by strongly diluting the serum and passing carbonic acid through it for a long time, when it is precipitated as a viscous deposit.

Fatty matter exists in small proportion, except in pathologic conditions and after the ingestion of food rich in oleaginous matters; it soon disappears, undergoing oxidation, generating heat and force, or is deposited as adipose tissue.

Sugar is represented by glucose, a product of the digestion of saccharine matter and starches in the alimentary canal; glycogenic matter is derived from the liver.

The saline constituents aid the process of osmosis, give alkalinity to the blood, promote the absorption of carbonic acid from the tissues into the blood, and hold other substances in solution; the most important are the sodium and potassium chlorids and the calcium and magnesium phosphates.

Excrementitious matters are represented by carbonic acid, urea, creatin, creatinin, urates, oxalates, etc. ; they are absorbed from the tissues by the blood and conveyed to the excretory organs, lungs, kidneys, etc.

Gases.-Oxygen, nitrogen, and carbonic acid exist in varying proportions. 


\section{BLOOD-CORPUSCLES.}

The corpuscular elements of the blood occur under two distinct forms, which, from their color, are known as the red and white corpuscles.

The red corpuscles as they float in a thin layer of the liquor sanguinis are of a pale straw-color; it is only when aggregated in masses that they assume the bright red color. In form they are circular and biconcave; they have an average diameter of $\frac{1}{3} \frac{1}{200}$ of an inch.

In mammals, birds, reptiles, amphibia, and fish the corpuscles vary in size and number, gradually becoming larger and less numerous as the scale of animal life is descended, e. $g$. :

TABLE SHOWING COMPARATIVE DIAMETER OF RED CORPUSCI.ES.

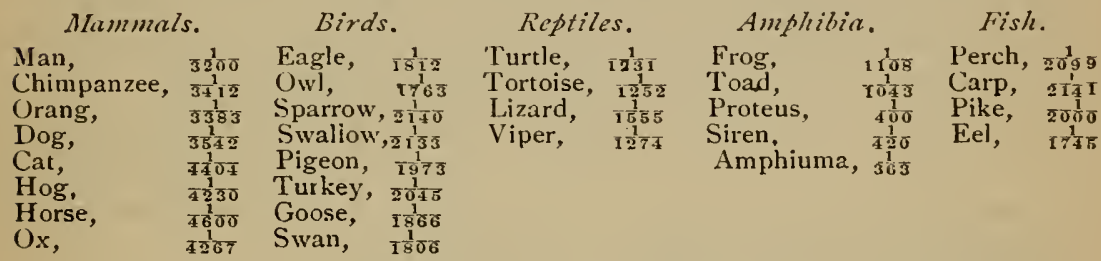

In man and the mammals the red corpuscles present neither a nucleus nor a cell wall, and are universally of a small size. They can be readily distinguished from the corpuscles of birds, reptiles, and fish, in which animals they are larger, oval in shape, and possess a well-defined nucleus.

The red corpuscles are exceedingly numerous, amounting to about $5,000,000$ in a cubic millimeter of blood. In structure they consist of a firm, elastic, colorless framework, - the stroma,-in the meshes of which is entangled the coloring-matter-the hemoglobin.

CHEMIC COMPOSITION OF RED CORPUSCI.ES.

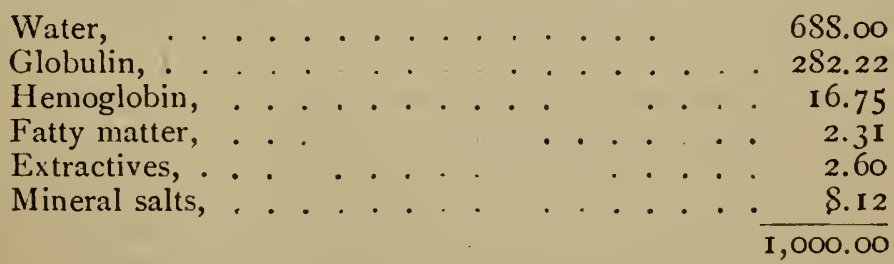

Hemoglobin, the coloring matter of the corpuscles, is an albuminous compound, composed of $\mathrm{C}, \mathrm{O}, \mathrm{H}, \mathrm{N}, \mathrm{S}$, and iron. It may exist in either an amorphous or a crystalline form. When deprived of all its oxygen, except the quantity entering into its intimate composition, the hemoglobin 
becomes purplish in color, and is known as reduced hemoglobin. When exposed to the action of oxygen, it again absorbs a definite amount and becomes scarlet in color, and is known as oxyhemoglobin. The amount of oxygen absorbed is 1.76 c.c. ( $\frac{7}{50}$ of a cubic inch) for I mg. ( $\frac{1}{64}$ of a grain) of hemoglobin.

It is this substance which gives the color to the venous and arterial blood. As the venous blood passes through the capillaries of the lungs the reduced hemoglobin absorbs the oxygen from the pulmonary air and becomes oxyhemoglobin, scarlet in color; the blood becomes arterial. When the arterial blood passes into the systemic capillaries, the oxygen is absorbed by the tissues; the hemoglobin becomes reduced, purple in color, and the blood becomes venous. A dilute solution of oxyhemoglobin gives two absorption bands between the lines $\mathrm{D}$ and $\mathrm{E}$ of the solar spectrum. Reduced hemoglobin gives but one absorption band, occupying the space existing between the two bands of the oxylhemoglobin spectrum.

The function of the red corpuscle is, therefore, to absorb oxygen and carry it to the tissues; the smaller the corpuscles and the greater the number, the greater is the quantity of oxygen absorbed, and, consequently, all the vital functions of the body become more active.

The white corpuscles are far less numerous than the red, the proportion being, on an average, about I white to from 350 to 400 red; they are globular in shape, and are $\frac{1}{2 \frac{1}{50}}$ of an inch in diameter, and consist of a soft, granular, colorless substance, containing several nuclei.

The white corpuscles possess the power of spontaneous movement, alternately contracting and expanding, throwing out processes of their substance and quickly withdrawing them, thus changing their shape from moment to moment. These movements resemble those of the ameba, and for this reason are termed ameboid. The white corpuscles also possess the capability of moving from place to place. In the interior of the vessels they adhere to the inner surface, while the red corpuscles move through the center of the stream.

The white corpuscles are identical with the leukocytes, and are found in milk, lymph, chyle, and other fluids.

Origin of Corpuscles.-The red corpuscles take their origin from the mesoblastic cells in the vascular area of the developing embryo.

In the adult they are produced from colorless, nucleated corpuscles resembling the white corpuscles. The spleen is the organ in which they are finally destroyed.

The white corpuscles originate from the leukocytes of the adenoid tissue, 
and subsequently give rise to the red corpuscles; they assist in the formation of the new tissues that result from inflammatory action.

\section{COAGULATION OF THE BLOOD.}

When blood is withdrawn from the body and allowed to remain at rest, it becomes somewhat thick and viscid in from three to five minutes; this viscidity gradually increases until the entire volume of blood assumes a jelly. like consistence, which process occupies from five to fifteen minutes.

As soon as coagulation is completed, a second process begins, which consists in the contraction of the coagulum and the oozing of a clear, straw-colored liquid, - the serum, - which gradually increases in quantity as the clot diminishes in size, by contraction, until the separation is completed, which occupies from twelve to twenty-four hours.

The changes in the blood are as follows :

Before coagulation.

Living blood. $\left\{\begin{array}{l}\text { Liq. Sanguinis, } \\ \text { or } \\ \text { Plasma, } \\ \text { Corpuscles, red and white. }\end{array}\right\}$ consisting of $\left\{\begin{array}{l}\text { Water. } \\ \text { Albumin. } \\ \text { Fibrinogen. } \\ \text { Salts. }\end{array}\right.$ After coagulation.

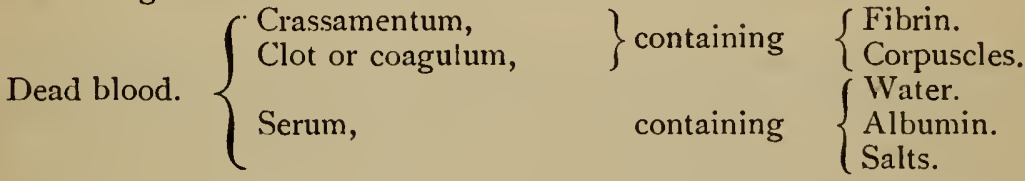

The serum, therefore, differs from the liquor sanguinis in not containing fibrin.

In from twelve to twenty-four hours the upper surface of the clot presents a grayish appearance, - the buffy coat, - owing to the rapid sinking of the red corpuscles beneath the surface, permitting the fibrin to coagulate without them; this substance then assumes a grayish-yellow tint. Inasmuch as the white corpuscles possess a lighter specific gravity than the red, they do not sink so rapidly, and, becoming entangled in the fibrin, assist in forming the buffy coat. Continued contraction gives a cupped appearance to the surface of the clot.

Inflammatory states of the blood produce a marked increase in the buffed and cupped condition, on account of the aggregation of the corpuscles and their tendency to rapid sinking. 
Nature of Coagulation. - Coagulated fibrin does not preëxist in the blood, but is formed at the moment blood is withdrawn from the vessels. According to Denis, a liquid substance-plasmin - exists in the blood, which, when withdrawn from the circulation, decomposes into fibrin and metalbumin.

According to Schmidt, fibrin results from the union of fibrinoplastin (paraglobulin) and furinogen, brought about by the presence of a third substance, the fibrin-ferment.

According to Hammersten and others, the fibrin obtained from the blood after coagulation comes from the fibrinogen alone, the conversion being brought about by the presence of a ferment substance, paraglobulin in this case having nothing to do with the change. This view is supported by the fact that the quantity of fibrin obtained from the blood is never greater than the quantity of fibrinogen previously present. The origin of the ferment is obscure, but there is reason to believe that it comes from the injured vascular coats or from the breaking of the white corpuscles.

Conditions Influencing Coagulation. - The process is retarded by cold, retention within living vessels, neutral salts in excess, inflammatory conditions of the system, imperfect aëration, exclusion from air, etc.

It is accelerated by a temperature of $100^{\circ} \mathrm{F}$., contact with air, rough surfaces, and rest.

Blood coagulates in the body after the arrest of the circulation in the course of twelve to twenty-four hours ; local arrest of the circulation, from compression or a ligature, will cause coagulation, thus preventing hemorrhages from wounded vessels.

The composition of the blood varies in different portions of the body. The arterial differs from the venous, in being more coagulable; in containing more oxygen and less carbonic acid; in having a bright scarlet color, from the union of oxygen with hemoglobin. The purple hue of venous blood results from the deoxidation of the coloring-matter.

The blood of the portal vein differs in constitution, according to different stages of the digestive process; during digestion it is richer in water, albuminous matter, and sugar; occasionally it contains fat; corpuscles are diminished, and there is an absence of biliary substances.

The blood of the hepatic vein contains a larger proportion of red and white corpuscles; the sugar is augmented, while albumin, fat, and fibrin are diminished. 


\section{CIRCULATION OF THE BLOOD.}

The circulatory apparatus by which the blood is distributed to all portions of the body consists of a central organ, - - the heart,- -with which is connected a system of closed ressels known as arteries, capillaries, and vcins. Within this system the blood is kept, by the action of the heart, in continual movement, distributing nutritive matter to all portions of the body and carrying waste matters from the tissues to the various eliminating organs.

The heart is a hollow, muscular organ, pyramidal in shape, measuring about $51 / 2$ inches in length and about $31 / 2$ in breadth, weighing from to to 12 ounces in the male and from $S$ to 10 in the female. Situated in the thoracic cavity, between the lungs, its base is directed upward, backward, and to the right; its apex is directed downward and to the left.

Pericardium.- The heart is surrounded by a closed fibrous membrane called the pericardium. The inner surface of this membrane is lined by a serous membrane, which is also reflected over the surface of the heart; between the two surfaces of the serous membrane is found a small quantity of fluid (the pericardial fluid), which lubricates the surfaces and prevents friction during the movements of the heart. The interior of the heart is also lined by a serous membrane, called the endocardium.

Cavities of the Heart.-The general cavity of the heart is subdivided by a longitudinal septum into a right and left half; each of these cavities is in turn subdivided by a transverse constriction into two smaller cavities, which communicate with each other and are known as the auricles and ventricles, the orifice between the auricle and ventricle being known as the auriculoventricular orifice. The heart, therefore, consists of four cavities - a right auricle and ventricle and a left auricle and ventricle.

Into the right auricle the two terminal trunks of the venous system-the superior and inferior vence cava-empty the venous blood which has been collected from all parts of the system; from the right ventricle arises the pulmonary artery, which, passing into the lungs, distributes the blood to the walls of the air-cells of the lungs; into the left auricle empty four pulmonary veins, which have collected the blood from the lung capillaries; from the left ventricle springs the aorta, the general trunk of the arterial system, the branches of which distribute the blood to the entire system. 


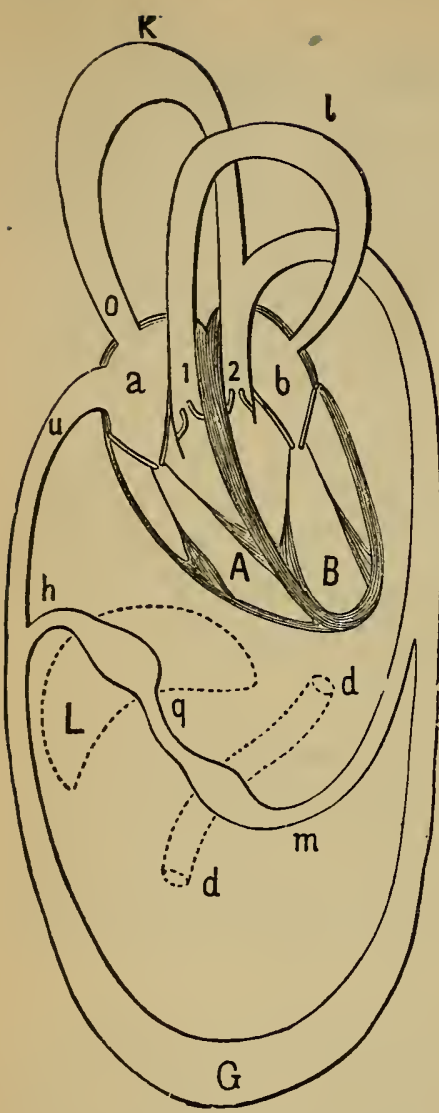

Fig. 17. - Scheme of the CirCUI.ATION.-(Landois.)

a Right, b. left, auricle. A. Right, $B$, left ventricle. I. Pulmonary artery. 2. Aorta. 1. Area of pulmonary, $\mathrm{K}$, area of systemic, circulation. o. The superior vena cava. G. Area supplying the inferior vena cava, u. d, d. Intestine. m. Mesenteric artery. q. Portal vein. L. Liver. h. Hepatic vein.
The Valves of the Heart.-The valves of the heart are formed by a reduplication of the endocardium strengthened by connective tissue. At the auriculoventricular openings on the right and left sides of the heart, respectively, are found the tricuspid and mitral valves. The tricuspid valve consists of three, the mitral of two, cusps or segments, which project into the interior of the ventricle when it does not contain blood. At their bases the segments are united so as to form an annular membrane attached to the margin of the orifice. To the free edges of the valves are attached numerous fine threads, - the chord a tendinece,which are the tendons of the small papillary muscles springing from the walls of the ventricles.

The Semilunar Valves.-At the openings of the pulmonary artery and the aorta are found three cup shaped or semilunar valves, the free edgcs of which are directed away from the interior of the heart. The anatomic arrangement of the valves is such that upon their closure regurgitation of the blood is prevented.

The Course of the Blood through the Heart.-Reference to figure $\mathbf{I} 7$ will make it clear that there is a pathway for the blood between the venæ cavæ on the right side and the aorta on the left side by way of the right side of the heart, the cardio-pulmonary vessels and the left side of the heart.

The venous blood flowing towards the heart is emptied by the superior and inferior venæ cavæ into the right auricle from which it passes through the auriculoventricular opening into the right ventricle; thence into and through the pulmonary artery and its branches to the pulmonary capillaries where it 
is arterialized, $i . e$., yields up its carbon dioxid and takes on a fresh supply of oxygen-and is changed in color from dark blue to scarlet red. The arterialized blood flowing towards the heart is emptied by the pulmonary veins into the left auricle from which it passes through the auriculoventricular opening into the left ventricle; thence into the aorta and its branches to the systemic capillaries where it is de-arterialized by an opposite exchange of gases, $i . e$., yields up a portion of its oxygen to, and absorbs carbon dioxid from the tissues, and changes in color from scarlet to dark blue. The venous blood is again returned by the systemic veins to the vena cavæ.

While there is but one circulation, physiologists frequently divide the circulatory apparatus into-

1. The systemic circuiation, which includes the movement of the blood from the left side of the heart through the aorta and its branches, through the capillaries and veins, to the right side.

2. The pulmonary circulation, which includes the course of the blood from the right side through the pulmonary artery, through the capillaries of the lungs and pulmonary veins, to the left side of the heart.

3. The portal circulation, which includes the portal vein. This vein is formed by the union of the radicles of the gastric, mesenteric, and splenic veins, and carries the blood directly into the liver, where the vein divides into a fine capillary plexus, from which the hepatic veins arise; these empty into the ascending vena cava.

The Mechanism of the Heart.-The immediate cause of the movement of the blood through the blood-vessels is the alternate contraction and relaxation of the muscular walls of the heart, and more especially the walls of the ventricles, each of which plays alternately the part of a force pump and to a slight extent of a suction pump. The motive power is furnished by the heart itself. The contraction of any part of the heart is termed the systole, the relaxation, the diastole; as each side of the heart has two cavities, the walls of which contract and relax in succession, it is customary to speak of an auricular systole and diastole and a ventricular systole and diastole ; as the two sides are in the same physiologic relation they contract and relax in the same periods of time.

Movements of the Heart. - At each beat, during the systole, the heart hardens and becomes shortened in its long diameter; its apex is raised up, rotated on its axis from left to right, and thrown forward against the walls of the chest. The impulse of the heart, observed about two inches below the nipple and one inch to the sternal side, between the fifth and sixth ribs, is caused mainly by the apex of the heart striking against 
the chest walls, assisted by the distention of the great vessels about the base of the heart.

The Cardiac Cycle.-The entire period of the heart's pulsation may be divided into three stages, viz. :

I. The auricular contraction and relaxation.

2. The ventricular contraction and relaxation.

3. The pause or period of repose during which both auricles and ventricles are at rest. These three stages constitute collectively a cardiac cycle or a cardiac revolution.

The duration of a cycle, as well as the duration of its three stages, varies in different animals in accordance with the number of cycles which recur in a minute. In human beings in adult life there are about 72 cycles to the minute; the average duration is, therefore, $0.80 \mathrm{sec}$. From this it follows that the time occupied by any one of the three stages must be extremely short and difficult of determination. From experiments on animals and from observations made on human beings, the following estimates have been made and accepted as approximately correct for human beings :

I. The auricular systole-0.16 sec.; the auricular diastole, $0.64 \mathrm{sec}$.

2. The ventricular systole $-0.32 \mathrm{sec}$; the ventricular diastole, $0.48 \mathrm{sec}$.

3. The period of rest for both auricles and venticles- $0.32 \mathrm{sec}$.

The Action of the Valves. - The forward movement of the blood is permitted and regurgitation prevented by the alternate action of the auriculoventricular and semilunar valves as a point of departure for a consideration of the action of these valves and their relation to the systole and diastole of the heart, the close of the ventricular systole may be selected. At this moment, the semilunar valves, which during the systole, are directed outward by the blood current are now suddenly and completely closed by the pressure of the blood in the aorta and pulmonary artery. Regurgitation into the ventricles is thus prevented.

During the ventricular systole, the relaxed auricles are filling with blood. With the ventricular diastole this blood or its equivalent flows into the relaxed and easily distensible ventricles until both auricles and ventricles are nearly filled. The tricuspid and mitral valves which are hanging down into the ventricular cavities, are now floated up by currents of blood welling up behind them until they are nearly closed. The auricles now suddenly contract, forcing their contained volumes into the ventricles which become fully distended.

With the cessation of the auricular systole, the ventricular systole begins. 
If the blood is not to be returned to the auricles the tricuspid and mitral valves must be instantly and completely closed. This is accomplished by the upward pressure of the blood which brings their free edges in close apposition. Reversal of these valves is prevented by the contraction of the papillary muscles which exert a traction on their under surfaces and edges and hold them steady.

The blood now confined in the ventricles between the closed auriculoventricular and semilunar valves is subjected to pressure on all sides; as the pressure rises proportionately to the vigor of the contraction there comes a moment when the intra-ventricular pressure exceeds that in the aorta and pulmonary artery; at once the semilunar valves are thrown open and the blood discharged. Both contraction and outflow continue until the ventricles are practically empty, when relaxation sets in attended by a rapid fall of pressure, under the influence of the positive pressure of the blood in the aorta and pulmonary artery, the semilunar valves are again closed. The accumulation of blood in the auricles, attended by a rise in pressure, again -forces the tricuspid and mitral valves open. With these events the cardiac cycle is again completed.

Sounds of the Heart.-It the ear be placed over the cardiac region, two distinct sounds are heard during each revolution of the heart, closely following each other, and which differ in character.

The sound coinciding with the systole in point of time-the first soundis prolonged and dull, and caused by the closure and vibration of the auriculoventricular valves, the contraction of the walls of the ventricles, and the apex-beat; the second sound, occurring during the diastole, is short and sharp, and caused by the closure of the semilunar valves.

The frequency of the heart's action varies at different periods of life, but in the adult male it beats about seventy-two times a minute. It is influenced by age, exercise, posture, digestion, etc.

Age.-Before birth, the number of pulsations a minute averages . . I40 During the first year it diminishes to ....... I28 During the third year diminishes to . . . . . . . 95 From the eighth to the fourteenth year averages . . . . . 84 In adult life the average is ........... . 72

Exercise and digestion increase the frequency of the heart's action.

Posture influences the number of pulsations a minute; in the male, standing, the average is $8 \mathrm{I}$; sitting, $7 \mathrm{I}$; lying, 66-independent, for the most part, of muscular effort.

The rhythmic movements of the heart are dependent upon- 
I. An inherent irritability of the muscle-fiber, which manifests itself as long as the nutrition is maintained.

2. The continuous flow of blood through its cavities, distending them and stimulating the endocardium.

The force exerted by the left ventricle at each contraction has been estimated at fifty-two pounds. If a tube be inserted into the aorta, the pressure there will be sufficient to support a column of blood nine feet, or a column of mercury six inches, in height, the weight in either case being about four pounds. The estimation of the force which the heart is required to exert to support this column of blood is arrived at by multiplying the pressure in the aorta (four pounds) by the area of the internal surface of the left ventricle (about thirteen inches), each inch of the ventricle being capable of supporting a downward pressure of four pounds.

Work Done by the Heart. - The work done by the heart is estimated by multiplying the amount of blood sent out from the right and left ventricles at each contraction by the pressure of the pulmonary artery and aorta, respectively-e.g., when the right ventricle contracts, it forces out $1 / 4$ of a pound of blood, and in so doing must overcome a pressure in the pulmonary artery sufficient to support a column of blood three feet in height; that is, must exert energy sufficient to raise $1 / 4$ of a pound 3 feet, or $1 / 4 \times 3$, or $3 / 4$ of a pound $I$ foot. When the left ventricle contracts, it sends out $1 / 4$ of a pound of blood, and in so doing the left ventricle must . overcome a pressure in the aorta sufficient to support a column of blood 9 feet in height ; that is, must exert energy sufficient to raise $1 / 4$ of a pound 9 feet, or $1 / 4 \times 9$, or $2 \frac{1}{4}$ pounds I foot. Work done is estimated by the amount of energy required to raise a definite weight a definite height; the unit, the foot-pound, being that required to raise I pound I foot.

The heart, therefore, at each systole exerts energy sufficient to raise 3 foot-pounds, and as it contracts 72 times a minute, it would raise in that time $3 \times 72$, or 216 foot-pounds; and in one hour $216 \times 60$, or 12,960 foot-pounds; and in twenty-four hours $12,960 \times 24$, or 311,040 footpounds, or 138.5 foot-tons.

Influence of the Nervous System upon the Heart.-When the heart of a frog is removed from the body, it continues to beat for a variable length of time, depending upon the nature of the conditions surrounding it. The heart of a warm-blooded animal continues to beat but for a very short time. The cause of the continued pulsations of the frog-heart is the presence of nerve-ganglia in its substance. These ganglia have not been 
shown to exist in the mammalian heart, but there is reason to believe that the nervous mechanism is fundamentally the same.

The ganglia of the heart are three in number: one situated at the opening of the inferior vena cava (the ganglion of Remak), a second situated in the auriculoventricular septum (the ganglion of Biddle), and a third situated in the interauricular septum (the ganglion of Ludwig). The first two are motor in function and excite the pulsations of the heart; the third is inhibitory in function and retards the action of the heart. The actions of these ganglia, though for the most part automatic, are modified by impressions coming through nerves from the medulla oblongata. When the inhibitory center is stimulated by muscarin, the heart is arrested in diastole; when atropin is applied, the heart recommences to beat, because atropin paralyzes the inhibitory center.

The nerves modifying the action of the heart are the pneumogastric (vagus) and the accelerator nerves.

The pneumogastric nerve, after emerging from the medulla, receives motor fibers from the spinal accessory nerve. It then passes downward, giving off branches, some of which terminate in the inhibitory ganglion. Stimulation of the vagus, by increasing the activity of the inhibitory center, arrests the heart in diastole with its cavities full of blood; but as the stimulation is only temporary, after a few seconds the heart recommences to beat; at first the pulsations are weak and feeble, but they soon regain their original vigor. After the administration of atropin in sufficient doses to destroy the termination of the pneumogastric, stimulation of its trunk has no effect upon the heart. The inhibitory fibers in the vagus are constantly in action, for division of the nerve on both sides is always followed by an increase in the frequency of the heart's pulsations.

The accelerator fibers arise in the medulla, pass down the cord, emerge in the cervical region, pass to the last cervical and first dorsal ganglia of the sympathetic, and thence to the heart. Stimulation of these fibers causes an increased frequency of the heart's pulsations, but they are dininished in force.

\section{ARTERIES.}

The arteries are a series of branching tubes conveying blood to all portions of the body. They are composed of three coats :

1. External, formed of areolar and elastic tissue.

2. Middle, contains both elastic and muscle fibers, arranged transversely to the long axis of the artery. The elastic tissue is more abundant in the larger vessels, the muscular in the smaller. 
3. Internal, composed of a thin, homogeneous membrane, covered with a layer of elongated endothelial cells.

The arteries possess both elasticity and contractility.

The property of elasticity allows the arteries already full to accommodate themselves to the incoming amount of blood, and to convert the intermittent acceleration of blood in the large vessels into a steady and continuous stream in the capillaries.

The contractility of the smaller vessels equalizes the current of blood, regulates the amount going to each part, and promotes the onward flow of blood.

Blood-pressure.-Under the influence of the ventricular systole, the recoil of the elastic walls of the arteries, and the resistance offered by the capillaries, the blood is constantly being subjected to a certain amount of pressure. If a large artery of an animal be divided, and a glass tube of the same caliber be inserted into its lumen, the blood will rise to a height of about nine feet; or if it be connected with a mercurial manometer, the mercury will rise to a height of six inches. This height will be a measure. of the pressure in the vessel. The absolute quantity of mercury sustained by an artery can be ascertained by multiplying the height of the column by the area of a transverse section of that artery.

The pressure of the blood is greatest in the large arteries, but gradually decreases toward the capillaries.

The blood-pressure is increased or diminished by influences acting upon the heart or upon the peripheral resistance of the capillaries, viz.:

If, while the force of the heart remains the same, the number of pulsations a minute increases, thus increasing the volume of blood in the arteries, the pressure rises. If the rate remains the same, but the force increases, the pressure again rises. Causes that increase the peripheral resistance by contracting the arterioles-e.g., irritation of the vasomotor nerves, cold, etc. - produce an increase of the pressure.

On the other hand, influences which diminish either the volume of the blood, or the number of pulsations, or the force of the heart, or the peripheral resistance, lower the pressure.

The pulse is the sudden distention of the artery in a transverse and longitudinal direction, due to the injection of a volume of blood into the arteries at the time of the ventricular systole. As the vessels are already full of blood, they must expand in order to accommodate themselves to the incoming volume of blood. The blood-pressure is thus increased, and the pressure originating at the ventricle excites a pulse-zwave, which passes from 
the heart toward the capillaries at the rate of about twenty-nine feet a second. It is this wave that is recognized by the finger.

The velocity with which the blood flows in the arteries diminishes from the heart to the capillaries, owing to an enlargement in the united sectional area of the vessels; the velocity increases from the capillaries toward the heart. The blood moves most rapidly in the large vessels, and especially under the influence of the ventricular systole. From experiments on animals, it has been estimated to move in the carotid of man at the rate of sixteen inches a second, and in the large veins at the rate of four inches a second.

The caliber of the blood-vessels is regulated by the vasomotor nerves, which have their origin in the gray matter of the medulla oblongata. They issue from the spinal cord through the anterior roots of spinal nerres, pass through the sympathetic ganglia, and ultimately are distributed to the coats of the blood-vessels. They exert at different times a constricting and a dilating action upon the vessels, thus keeping up the arterial tonus.

Capillaries.-The capillaries constitute a network of vessels of microscopic size, which distribute the blood to the inmost recesses of the tissues, inosculating with the arteries on the one hand and the veins on the other; they branch and communicate in every possible direction.

The diameter of a capillary vessel varies from $\frac{1}{6000}$ to $\frac{1}{3000}$ of an inch ; the walls of these consist of a delicate, homogeneous membrane, $\frac{1}{2000} \overline{0}$ of an inch in thickness, lined by flattened, elongated, endothelial cells, between which, here and there, are observed stomata.

It is through the agency of the capillary vessels that the phenomena of nutrition and secretion take place, for here the blood flows in an equable and continuous current, and is brought into intimate relationship with the tissues - two of the essential conditions for proper nutrition.

The rate of movement in the capillary vessels is estimated at one inch in thirty seconds.

In the capillary current the red corpuscles may be seen hurrying down the center of the stream, while the white corpuscles in the still layer adhere to the walls of the vessel, and at times can be seen to pass through the walls of the vessel by ameboid movements.

The passage of the blood through the capillaries is mainly due to the force of the ventricular systole and the elasticity of the arteries; but it is probably also aided by a power resident in the capillaries themselves, the result of a vital relation between the blood and the tissues.

The veins are the vessels which return the blood to the heart; they 
have their origin in the venous radicles, and as they approach the heart converge to form larger trunks, and terminate finally in the venæ cavæ.

They possess three coats-

I. External, made up of areolar tissue.

2. Middle, composed of non-striated muscle-fibers; yellow, elastic, and fibrous tissue.

3. Internal, an endothelial membrane similar to that of the arteries.

Veins are distinguished by the possession of valves throughout their course, which are arrunged in pairs, and formed by a reflection of the internal coat, strengthened by fibrous tissues; they always look toward the heart, and when closed prevent a reflux of blood in the veins. Valves are most numerous in the veins of the extremities, but are entirely absent in many others.

The onward flow of blood in the veins is mainly due to the action of the heart, but is assisted by the contraction of the voluntary muscles and the force of respiration.

Muscular contraction, which is intermittent, aids the flow of blood in the veins by compressing them. As regurgitation is prevented by the closure of the valves, the blood is forced onward toward the heart.

Rhythmic movements of veins have been observed in some of the lower animals, aiding the onward current of blood.

During the movement of inspiration the thorax is enlarged in all its diameters, and the pressure on its contents at once diminishes. Under these circumstances a suction force is exerted upon the great venous trunks, which causes the blood to flow with increased rapidity and volume toward the heart.

Venous Pressure.-As the force of the heart-beat is nearly expended in driving the blood through the capillaries, the pressure in the venous system is not very marked, not amounting in the jugular vein of. a dog to more than $\frac{1}{1} \overline{2}$ that of the carotid artery.

'The time required for a complete circulation of the blood throughout the vascular system has been estimated to be from twenty to thirty seconds, while for the entire mass of blood to pass through the heart fifty-eight pulsations would be required, occupying forty-eight seconds.

The forces keeping the blood in circulation are:

1. Action of the heart.

2. Elasticity of the arteries.

3. Capillary force.

4. Contraction of the voluntary muscles upon the veins.

5. Respiratory movements. 


\section{RESPIRATION.}

Respiration is the function by which oxygen is absorbed into the blood and carbonic acid exhaled. The assimilation of the oxygen and the evolution of carbonic acid takes place in the tissues as a part of the general nutritive process, the blood and respiratory apparatus constituting the media by means of which the interchange of gases is accomplished.

The respiratory apparatus consists of a larynx, trachea, and lungs.

The larynx is composed of firm cartilages, united by ligaments and muscles. Running anteroposteriorly across the upper opening are four ligamentous bands, - the two superior or false vocal cords, and the two inferior or true vocal cords, - formed by folds of the mucous membrane. They are attached anteriorly to the thyroid cartilages and posteriorly to the arytenoid cartilages, and are capable of being separated by the contraction of the posterior crico-arytenoid muscles, so as to admit the passage of air into and from the lungs.

The trachea is a tube from four to five inches in length, $\frac{3}{4}$ of an inch in diameter, extending from the cricoid cartilage of the larynx to the third dorsal vertebra, where it divides into the right and left bronchi. It is composed of a series of cartilaginous rings, which extend about two thirds around its circumference, the posterior third being occupied by fibrous tissue and non-striated muscle-fibers; which are capable of diminishing its caliber.

The trachea is covered externally by a tough, fibro-elastic membrane, and internally by mucous membrane, lined by columnar, ciliated, epithelial cells. The cilia are always waving from within outward. When the two bronchi enter the lungs, they divide and subdivide into numerous smaller branches, which penetrate the lungs in every direction until they finally terminate in the pulmonary lobules.

As the bronchial tubes become smaller their walls become thinner; the cartilaginous rings disappear, but are replaced by irregular angular plates of cartilage; when the tube becomes less than $\frac{1}{50}$ of an inch in diameter, they wholly disappear, and the fibrous and mucous coats blend, forming a delicate elastic membrane, with circular muscle-fibers.

The lungs occupy the cavity of the thorax, are conic in shape, of a pink color and a spongy texture. They are composed of a great number of distinct lobules (the pulmonary lobules), connected by interlobular connective tissue. These lobules vary in size, are of an oblong shape, and 
are composed of the ultimate ramifications of the bronchial tubes, within which are contained the air-vesicles or cells. The walls of the air-vesicles, exceedingly thin and delicate, are lined internally by a layer of tessellated epithelium, externally covered by elastic fibers, which give the lungs their elasticity and distensibility.

The venous blood is distributed to the lungs for aeration by the pulmonary artery, the terminal branches of which form a rich plexus of capillary vessels surrounding the air-cells; the air and blood are thus brought into intimate relationship, being separated only by the delicate walls of the aircells and capillaries.

The thoracic cavity, in which the respiratory organs are lodged, is of a conic shape, having its apex directed upward, its base downward. Its framework is formed posteriorly by the spinal column, anteriorly by the sternum, and laterally by the ribs and costal cartilages. Between and over the ribs lie muscles, fascia, and skin, above, the thorax is completely closed by the structures passing into it and by the cervical fascia and skin; below, it is closed by the diaphragm. It is, therefore, an air-tight cavity.

The Pleura.-Each lung is surrounded by a closed serous membrane (the pleura), one layer of which (the visceral) is reflected over the lung; the other (the parietal), reflected over the wall of the thorax; between the two layers is a small amount of fluid, which prevents friction during the play of the lungs in respiration.

Owing to the elastic tissue which is present in the lungs, they are very readily distensiblę; so much so, indeed, that the pressure of the air inside the trachea and lungs is sufficient to distend them until they completely fill all parts of the thoracic cavity not occupied by the heart and great vessels. The elastic tissue endows them not only with distensibility, but also with the power of elastic recoil, by which they are enabled to accommodate themselves to all variations in the size of the thoracic cavity.

When the chest-walls recede, the air within the lungs expands and presses them against the ribs; when the chest-walls contract, the air being driven out, the elastic tissue recoils and the lungs return to their original condition. The movements of the lungs are, therefore, entirely passive.

As the capacity of the chest in a state of rest is greater than the volume of the lungs after they are collapsed, it is quite evident that in the living condition the lungs are distended and in a state of elastic tension, which is greater or less in proportion as the thoracic cavity is increased or diminislied in size. The elastic tissue, always on the stretch, is endeavoring to pull the visceral layer of the pleura away from the parietal layer, but is 
antagonized by the pressure of the air within the air-passages. This condition of things persists as long as the thoracic cavity remains air-tight ; but if an opening be made in the thoracic wall, the pressure of the external air, which was previously supported by the practically rigid walls of the thorax, now presses upon the lung with as much force as the air within the lung. The two pressures being neutralized, there is nothing to prevent the elastic tissue from recoiling, driving the air out, and collapsing. 'The elastic tension of the lungs can be readily measured in man after death by inserting a manometer into the trachea. Upon opening the thorax and allowing the tissue to recoil, the air passes upon the mercury and elevates it, the extent to which it is raised being the index of the pressure. Hutchinson calculated the pressure to be one half pound to the square inch of lung surface.

\section{Respiratory Movements. - The} movements of respiration are two, and consist of an alternate dilatation and contraction of the chest, known as inspiration and expiration.

I. Inspiration is an active process, the result of the expansion of the thorax, whereby air is introduced into the lungs.

2. Expiration is a partially passive process, the result of the recoil of the elastic walls of the thorax, and the recoil of the elastic tissue of the

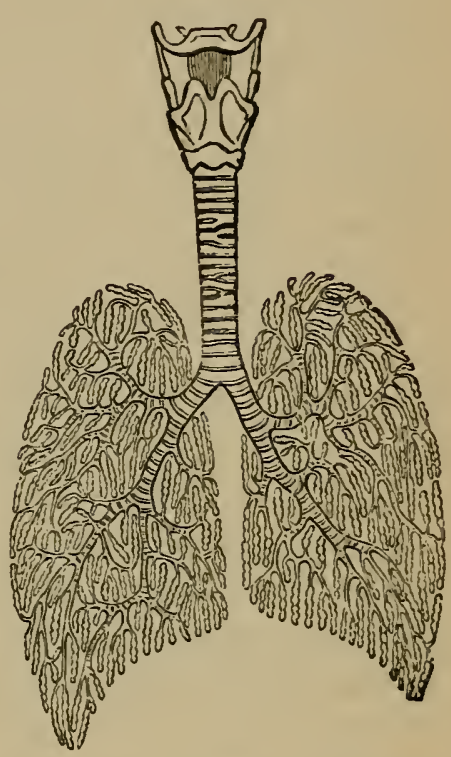

Fig. 18.-Diagram of the RespiRATORY ORgaNs.

The windpipe, leading down from the larynx, is seen to branch into two large bronchi, which subdivide after they enter their respective lungs. lungs, whereby the carbonic acid is expelled.

In inspiration the chest is enlarged by an increase in all its diameters viz. :

I. The vertical is increased by the contraction and descent of the diaphragm when it approximates a straight line.

2. The anteroposterior and t,ansverse diameters are increased by the elevation and rotation of the ribs upon their axes.

In ordinary tranquil inspiration the muscles which elevate the ribs and 
thrust the sternum forward, and so increase the diameters of the chest, are the external intercostals, running from above downward and forward, the sternal portion of the internal intercostals, and the levatores costarum.

In the extraordinary efforts of inspiration certain auxiliary muscles are brought into play, - viz., the sternomastoid, pectorales, serratus magnus, which increase the capacity of the thorax to its utmost limit.

In expiration the diameters of the chest are all diminished-viz. :

I. The vertical, by the ascent of the diaphragm.

2. The anteroposterior, by a depression of the ribs and sternum.

In ordinary tranquil expiration the diameters of the thorax are diminished by the recoil of the elastic tissue of the lungs and the ribs; but in forcible expiration the muscles which depress the ribs and sternum, and thus further diminish the diameter of the chest, are the internal intercostals, the infracostals, and the triangularis sterni.

In the extraordinary efforts of expiration certain auxiliary muscles are brought into play, - viz., the abdominal and sacrolumbalis muscles, - which diminish the capacity of the thorax to its utmost limit.

Expiration is aided by the recoil of the elastic tissue of the lungs and ribs and by the pressure of the air.

Movements of the Glottis.-At each inspiration the rima glottidis is dilated by a separation of the vocal cords, produced by the contraction of the crico-arytenoid muscles, so as freely to admit the passage of air into the lungs; in expiration they fall passively together, but do not interfere with the exit of air from the chest.

Nervous Mechanism of Respiration. - The movements of respiratory muscles, though capable of being modified to a certain extent by efforts of the will, are of an automatic character, and called forth by nervous impulses emanating from the medulla oblongata. The respiratory center, the so-called vital point, generates the nerve impulses, which, traveling outward through the phrenic and intercostal nerves, excite contractions of the diaphragm and intercostal muscles, respectively. This center is for the most part automatic in its action, though it is capable of being modified by impulses reflected to it through various sensory nerves.

This center may be stimulated :

1. Directly, by the condition of the blood. An increase of carbonic acid or a diminution of oxygen in the blood causes an acceleration of the respiratory movements; the reverse of these conditions causes a diminution of the respiratory movements. 
2. Indirectly, by reflex action. The medulla may be excited to action through the pneumogastric nerve, by the presence of carbonic acid in the lungs irritating its terminal filaments; through the fifth nerve, by irritation of the terminal branches; and through the nerves of general sensibility. In either case this center retlects motor impulses to the respiratory muscles through the phrenic, intercostals, inferior laryngeal, and other nerves.

Types of Respiration.-The abdominal type is most marked in young children, irrespective of sex, the respiratory movements being effected by the diaphragm and abdominal muscles.

In the superior costal type, exhibited by the adult female, the respiratory movenients are more marked in the upper part of the chest, from the first to the seventh ribs, permitting the uterus to ascend in the abdomen during pregnancy without interfering with respiration.

In the inferior costal type, manifested by the male, the movements are largely produced by the muscles of the lower portions of the chest, from the seventh rib downward, assisted by the diaphragm.

The respiratory movements vary according to age, sleep, and exercise, being most frequent in early life, but averaging twenty a minute in adult life. They are diminished by sleep and increased by exercise. There are about four pulsations of the heart to each respiratory act.

During inspiration two sounds are produced: the one, heard in the thorax, in the trachea, and larger bronchial tubes, is tubular in character; the other, heard in the substance of the lungs, is vesicular in character.

\section{AMOUNT OF AIR EXCHANGED IN RESPIRATION, AND CAPACITY OF LUNGS.}

The tidal or breathing volume of air, that which passes in and out of the lungs at each inspiration and expiration, is estimated at from twenty to thirty cubic inches.

The complemental air is that amount which can be taken into the lungs by a forced inspiration, in addition to the ordinary tidal volume, and amounts to about $\mathbf{I}$ Io cubic inches.

The reserve air is that which usually remains in the chest after the ordinary efforts of expiration, but which can be expelled by forcible expiration. The volume of reserve air is about roo cubic inches.

The residual air is that portion which remains in the chest and cannot be expelled after the most forcible expiratory efforts, and which amounts, according to Dr. Hutchinson, to about 100 cubic inches. 
The vital capacity of the chest indicates the amount of air that can be forcibly expelled from the lungs after the deepest possible inspiration, and is an index of an individual's power of breathing in disease and during prolonged severe exercise. The combined amount of the tidal, the complemental, and the reserve air, 230 cubic inches, represents the vital capacity of an individual five feet seven inches in height. The vital capacity varies chiefly with stature. It is increased eight cubic inches for every inch in height above this standard, and diminishes eight cubic inches for each inch below it.

The tidal volume of air is carried only into the trachea and large bronchial tubes by the inspiratory movements. It reaches the deeper portions of the lungs in obedience to the law of diffusion of gases, which is inversely proportionate to the square root of their densities.

The ciliary action of the columnar cells lining the bronchial tubes also assists in the interchange of air and carbonic acid.

The entire volume of air passing in and out of the thorax in twenty-four hours is subject to great variation, but can be readily estimated from the tidal volume and the number of respirations a minute. Assuming that an individual takes into the chest twenty cubic inches at each inspiration, and breathes eighteen times a minute, in twenty-four hours there would pass in and out of the lungs 518,400 cubic inches, or 300 cubic feet.

Chemistry of Respiration.-As the inspired air undergoes a change in composition during its stay in the lungs which renders it unfit for further respiration, it becomes requisite, for the correct understanding of respiration, to ascertain the composition of both inspired and expired air.

Composition of Air.-Chemic analysis has shown that every 100 volumes of air contain 20.8I volumes of oxygen, 70.19 volumes of nitrogen, and 0.03 volume of carbonic acid. Aqueous vapor is also present, though the quantity is variable. The higher the temperature, the greater the amount.

The changes in the air effected by respiration are:

Loss of oxygen, to the extent of five cubic inches per 100 of air, or one in twenty.

Gain of carbonic acid, to the extent of 4.66 cubic inches per 100 of air, or 0.93 inch in twenty.

Increase of water-vapor and organic matter.

Elevation of temperature.

Increase, and at times decrease, of nitrogen.

Gain of ammonia. 
The total yuantity of oxysen withdrawn from the air and consumed by the body in twenty-four hours amounts to fifteen cubic feet, and can be readily estimated from the amount consumed at each respiration. Assuming that one cubic inch of oxygen remains in the lungs at each respiration, in one hour there are consumed roSo cubic inches, and in twenty four hours 25,920 cubic inches, or fifteen cubic feet, weighing eighteen ounces. To obtain this quantity, 300 cubic feet of air are necessary.

The quantity of oxygen consumed daily is subject to considerable variations. It is increased by exercise, digestion, and lowered temperature, and decreased by the opposite conditions.

The quantity of carbonic acia' exhaled in twenty-four hours varies greatly. It can be estimated in the same way. Assuming that an individual exhales $0.93+$ cubic inch at each respiration, in one hour there are eliminated roo 8 cubic inches, and in twenty-four hours 24,192 cubic inches, or fourteen cubic feet, containing seven ounces of pure carbon.

The exhalation of carbonic acid is increased by muscular exercise, nitrogenous food, tea, coffee, and rice, age, and by muscular development; decreased by a lowering of temperature, repose, gin and brandy, and a dry condition of the air.

As there is always more oxygen consumed than carbonic acid exhaled, and as oxygen unites with carbon to form an equal volume of carbonic acid, it is evident that a certain quantity of oxygen disappears within the body. In all probability it unites with the sulphur hydrogen of the food to form water.

The amount of watery vapor which passes out of the body with the expired air is estimated at from one to two pounds.

The organic matter, though slight in amount, gives the odor to the breath. In a roon with defective ventilation the organic matter accumulates and gives rise to headache, nausea, drowsiness, etc. I.ong-continued breathing of such air produces general ill health. It is not so much the presence of $\mathrm{CO}_{2}$ in increased amount as the presence of organic matter which necessitates thorough ventilation.

\section{Condition of the Gases in the Blood.}

Oxygen is absorbed from the lungs into the arterial blood by the coloringmatter, hemoglobin, with which it exists in a state of loose combination, and is disengaged during the process of nutrition.

Carbonic acid, arising in the tissues, is absorbed into the blood in consequence of its alkalinity, where it exists in a state of simple solution and also in a state of feeble combination with the carbonates, soda and potassa, forming the bicarbonates. 
Nitrogen is simply beld in solution in the plasma.

Exchange of Gases in the Air-cells. - From the difference in tension of the oxygen in the air-cells $(27.44 \mathrm{~mm}$ of $\mathrm{Hg})$ and of the oxygen in the venous blood $(22 \mathrm{~mm}$. $\mathrm{Hg}$ ), and from the difference of the carbonic acid tension in the venous blood $(4 \mathrm{I} \mathrm{mm} . \mathrm{Hg})$ and in the air-cells $(27 \mathrm{~mm}$. $\mathrm{Hg}$ ), it might be concluded that the passage of the gases is due solely to pressure. The absorption of oxygen, however, does not follow absolutely the law of pressure; that chemic processes are involved is shown by the union of oxygen with the hemoglobin of the blood corpuscles. The exhalation of $\mathrm{CO}_{2}$ is also partly a chemic process, as it has been shown that the quantity excreted is greatly increased when oxygen is simultaneously absorbed. Oxygen not only favors the exhalation of loosely combined $\mathrm{CO}_{2}$, but favors the expulsion of that which can be excreted only by the addition of acids to the blood.

Changes in the Blood during Respiration.

As the blood passes through the lungs it is changed in color, from the dark purple of venous blood to the bright red of arterial blood.

The heterogeneous composition of venous blood is exchanged for the uniform composition of the arterial.

It gains oxygen and loses carbonic acid.

Its coagulability is increased. Temperature is diminished.

Asphyxia.-If the supply of oxygen to the lungs be diminished and the carbonic acid retained in the blood, the normal respiratory movements cease and the condition of asphyxia ensues, which soon terminates in death.

The phenomena of asphyxia are violent spasmodic action of the respiratory muscles attended by convulsions of the muscles of the extremities, engorgement of the venous system, lividity of the skin, abolition of sensibility and reflex action, and death.

The cause of death is a paralysis of the heart from overdistention by blood. The passage of the blood through the capillaries is prevented by contraction of the smaller arteries, from irritation of the vasomotor center. The heart is enfeebled by a want of oxygen and inhibited in its action by the inhibitory centers. 


\section{ANIMAL HEAT.}

The functional activity of all the organs and tissues of the body is attended by the evolution of heat, which is independent, for the most part, of external conditions. Heat is a necessary condition for the due performance of all vital actions; although the body constantly loses heat by radiation and evaporation, it possesses the capability of renewing it and of maintaining it at a fixed standard. The normal temperature of the body in the adult, as shown by means of a delicate thermometer placed in the axilla, ranges from $97.25^{\circ} \mathrm{F}$. to $99.5^{\circ} \mathrm{F}$., though the mean normal temperature is estimated by Wunderlich at $9^{\$} 6^{\circ} \mathrm{F}$.

The temperature varies in different portions of the body according to the extent to which oxidation takes place, being highest in the muscles, in the brain, blood, liver, etc.

The conditions which produce variations in the normal temperature of the body are: age, period of the day, exercise, food and drink, climate, season, and disease.

Age. - At birth the temperature of the infant is about $\mathrm{I}^{\circ} \mathrm{F}$. above that of the adult, but in a few hours falls to $95.5^{\circ} \mathrm{F}$, to be followed in the course of twenty-four hours by a rise to the normal or a degree beyond. During childhood the temperature approaches that of the adult; in aged persons the temperature remains about the same, though they are not so capable of resisting the depressing effects of external cold as adults. A diurnal varintion of the temperature occurs from $1.8^{\circ} \mathrm{F}$. to $3 \cdot 7^{\circ} \mathrm{F}$. (Jürgensen); the maximum occurring late in the afternoon, from 4 to 9 P. M. ; the mini$m u m$, early in the morning, from $\mathbf{I}$ to $7 \mathrm{~A}$. $M$.

Exercise. - The temperature is raised from $\mathrm{I}^{\circ}$ to $2^{\circ} \mathrm{F}$. during active contractions of the muscular masses, and is probably due to the increased activity of chemic changes; a rise beyond this point being prevented by its diffusion to the surface, consequent on a more rapid circulation, radiation, more rapid breathing, etc.

Food and Drink. - The ingestion of a hearty meal increases the temperature but slightly; an absence of food, as in starvation, produces a marked decrease. Alcoholic drinks, in large amounts, in persons unaccustomed to their use, cause a depression of the temperature amounting to. from $\mathrm{I}^{\circ}$ to $2^{\circ} \mathrm{F}$. Tea causes a slight elevation.

External Temperature. - Long-continued exposure to cold, especially if the body is at rest, diminishes the temperature from $\mathrm{I}^{\circ}$ to $2^{\circ} \mathrm{F}$., while exposure to a great heat slightly increases it. 
Disease frequently causes a marked variation in the normal temperature of the body, which rises as high as $107^{\circ} \mathrm{F}$. in typhoid fever and $105^{\circ} \mathrm{F}$. in pneumonia; in cholera it falls as low as $80^{\circ} \mathrm{F}$. Death usually occurs when the heat remains high and persistent, from $106^{\circ}$ to $110^{\circ} \mathrm{F}$; the increase of heat in disease is due to excessive production rather than to diminished elimination.

The source of heat is to be sought for in the chemic decompositions and hydrations taking place during the general process of nutrition, and in the combustion of the carbonaceous compounds by the oxygen of the inspired air ; the amount of its production is in proportion to the activity of the internal changes.

Every contraction of a muscle, every act of secretion, each exhibition of nerve force, is accompanied by a change in the chemic composition of the tissues and an evolution of heat. The reduction of the disintegrated tissues to their simplest form by oxidation, and the combination of the oxygen of the inspired air with the carbon and hydrogen of the blood and tissues, results in the formation of carbonic acid and water and the generation of a great amount of heat.

Certain elements of the food, particularly the non-nitrogenized substances, undergo oxidation without taking part in the formation of the tissues, being transformed into carbonic acid and water, and thus increase the sum of heat in the body.

Heat-producing Tissues.-All the tissues of the body add to the general amount of heat, according to the degree of their activity. But special structures, on account of their mass and the large amount of blood they receive, are particularly to be regarded as heat producers, e. g..

I. During mental activity the brain receives nearly one fifth of the entire volume of blood, and the venous blood returning from it is charged with waste matters, and its temperature is increased.

2. The muscular tissue, on account of the many chernic changes occurring during active contractions, must be regarded as the chief heat-producing tissue.

3. The secreting glands, during their functional activity, add largely to the amount of heat.

The entire quantity of heat generated within the body has been demonstrated experimentally to be about 2,300 calories, a calory, or heat unit, being that amount of heat required to raise the temperature of one kilogram of water $\left(2.2\right.$ pounds) $\mathrm{r}^{\circ} \mathrm{C}$. This quantity of heat, if not utilized and re- 
tained within the body, would elevate its temperature in twenty-four hours about $60^{\circ} \mathrm{F}$. That this volume of heat depends very largely upon the oxidation of the food-stuffs can be shown experimentally.

The normal temperature of the body is maintained by a constant expenditure of the heat in several directions:

r. In warming the food, drink, and air that are consumed in twenty-four hours. For this purpose about $\mathbf{I} 57$ heat units are required.

2. In evaporating water from the skin and lungs, 619 heat units being utilized for this purpose.

3. In radiation and conduction. By these processes the body loses at least fifty per cent. of its heat, or I, I 56 heat units.

4. In the production of work; the work of the circulatory, respiratory, muscular, and nervous apparatus being performed by the transformation of 369 heat units into units of work.

The nervous system influences the production of heat in a part by increasing the amount of blood passing through it by its action upon the vasomotor nerves. Whether there exists a special heat-center has not been satisfactorily determined, though this is probable.

\section{SECRETION.}

The process of secretion consists in the separation of materials from the blood which are either to be again utilized to fulfil some special purpose in the economy, or are to be removed from the body as excrementitious matter; in the former case they constitute the secretions, in the latter, the excretions.

The materials which enter into the composition of the secretions are derived from the nutritive principles of the blood, and require special organs-e. $g$., gastric glands, mammary glands, etc.-for their proper elaboration.

The materinls which compose the excretions preexist in the blood, and are the results of the activities of the nutritive process; if retained within the body, they exert a deleterious influence upon the composition of the blood.

Destruction of a secreting gland abolishes the secretion peculiar to it, and it can not be formed by any other gland; but among the excreting organs there exists a complementary relation, so that if the function of one organ be interfered with, another performs it to a certain extent. 


\section{CLASSIFICATION OF THE SECRETIONS.}

PERMANENT FLUIDS.

Serous fluids.

Synovial fluid.

Aqueous humor of the eye.
Vitreous humor of the eye.

Fluid of the labyrinth of the internal ear.

Cerebro-spinal fluid.

\section{TRANSITORY FLUIDS.}

Mucus.

Sebaceous matter.

Cerumen (external meatus).

Meibomian fluid.

Milk and colostrum.

Tears.

Saliva.
Gastric juice.

Pancreatic juice.

Secretion from Brunner's glands.

Secretion from Lieberkühn's glands. Secretions from follicles of the large intestine.

Bile (also an excretion).

\section{EXCRETIONS.}

Perspiration and the secretion of Urine. the axillary glands. Bile (also a secretion).

FLUIDS CONTAINING FORMED ANATOMIC ELEMENTS.

Seminal fluid, containing sperma- Fluid of the Graafian follicles. tozoids.

The essential apparatus for secretion is a delicate, homogeneous, structureless membrane, on one side of which, in close contact, is a capillary plexus of blood-vessels, and on the other side a layer of cells the physiologic function of which varies in different situations.

Secreting organs may be divided into membranes and glands.

Serous membranes usually exist as closed sacs, the inner surfaces of which are covered by pale, nucleated epithelium, containing a small amount of secretion.

The serous membranes are the pleura, peritoneum, pericardium, synovial sacs, etc.

The serous fuids are of a pale amber color, soinewhat viscid, alkaline, coagulable by heat, and resemble the serum of the blood; their amount is but small. The pleural fluid varies from four to seven drams; the peritoneal from one to four ounces; the pericardial from one to three drams.

The synovial fluid is colorless, alkaline, and extremely viscid, from the presence of synovin. 
The function of serous fluids is to moisten the opposing surfaces, so as to prevent friction during the play of the viscera.

The mucous membranes are soft and velvety in character, and line the cavities and passages leading to the exterior of the body-e.g., the gastrointestinal, pulmonary and genito-urinary. They consist of a primary basement membrane covered with epithelial cells, which in some situations are tessellated, in others, columnar.

Mucus is a pale, semitransparent, alkaline fluid, containing epithelial cells and leukocytes. It is composed, chemically, of water, an albuminous principle (mucosin), and mineral salts; the principal varieties are nasal, bronchial, vaginal, and urinary.

Secreting glands are formed of the same elements as the secreting membranes, but instead of presenting flat surfaces, are involuted, forming tubules, which may be simple follicles--e.g., mucous, uterine, or intestinal ; or compound follicles-e.g., gastric glands, mammary glands, or racemose glands-e. g., salivary glands and pancreas. They are composed of a basement membrane, enveloped by a plexus of blood-vessels, and are lined by epithelial and true secreting cells, which in different glands possess the capability of elaborating elements characteristic of their secretions.

In the production of the secretion two essentially different processes are concerned.

1. Chemic.-The formation and elaboration of the characteristic organic ingredients of the secreted fluids-e.g., pepsin, pancreatin-take place during the intervals of glandular activity, as a part of the general function of nutrition. They are formed by the cells lining the glands, and can often be seen in their interior with the aid of the microscope-e. $g$., bile in the liver cells, fat in the cells of the mammary gland.

2. Physical.-Consisting of a transudation of water and mineral salts from the blood into the interior of the gland.

During the intervals of glandular activity only that amount of blood passes through the gland sufficient for proper nutrition; when the gland begins to secrete, under the influence of an appropriate stimulus, the bloodvessels dilate and the quantity of blood becomes greatly increased beyond that flowing to the gland during its repose.

Under these conditions a transudation of water and salt takes place, washing out the characteristic ingredients, which are discharged by the gland ducts. The dischargre of the secretions is intermittent; they are retained in the glands until they receive the appropriate stimulus, when 
they pass into the larger ducts by the vis a tergo, and are then discharged by the contraction of the muscular walls of the ducts.

The activity of grandular secretion is hastened by an increase in the blood-pressure and retarded by a diminution.

The nervous centers in the medulla oblongata influence secretion :

I. By increasing or diminishing the amount of blood entering a gland.

2. By exerting a direct influence upon the secreting cells themselves, the centers being excited by reflex irritation, mental emotion, etc.

\section{MAMMARY GLANDS.}

The mammary glands, which secrete the milk, are two more or less hemispheric organs, situated in the human female on the anterior surface of the chest. Though rudimentary in childhood, they gradually increase in size as the young female approaches puberty.

The gland presents at its convexity a small prominence of skin (the nipple), which is surrounded by a circular area of pigmented skin (the areola). 'The gland proper is covered by a layer of adipose tissue anteriorly and is attached posteriorly to the pectoral muscles by a meshwork of fibrous tissue. During utero-gestation the mammary glands become larger, firmer, and more lobulated ; the areola darkens and the veins become more prominent. At the period of lactation the gland is the seat of active histologic and physiologic changes, correlated with the production of milk. At the close of lactation the glands diminish in size, undergo involution, and gradually return to their original non-secreting condition.

Structure of the Mammary Glands. - Each mammary gland consists of an aggregation of some fifteen or twenty lobes, each of which is surrounded by a framework of fibrous tissue. The lobe is provided with an excretory duct, which, as it approaches the base of the nipple, expands to form a sinus or reservoir, beyond which it opens by a narrowed orifice on the surface of the nipple. On tracing the duct into a lobe, it is found to divide and subdivide, and finally to terminate in lobules or acini. Each acinus consists of a basement membrane, lined by low polyhedral cells. Externally it is surrounded by connective tissue, supporting blood-vessels, lymphatics, and nerves.

\section{MILK.}

Milk is an opaque, bluish-white fluid, almost inodorous, of a sweet taste, an alkaline reaction, and a specific gravity of 1025 to 1040. When exam- 
ined microscopically it is seen to consist of a clear fluid (the milk-plasma), holding in suspension an enormous number of small, highly refractive oilglobules, which measure, on an average, 1000\% of an inch in diameter, Each globule is supposed by some observers to be surrounded by a thin. albuminous envelope, which enables it to maintain the discrete form. The quantity of milk secreted daily by the human female averages about two and a half pints. The milk of all the mammalia consists of all the different classes of nutritive principles, though in varying proportions. The relative proportions in which these constituents exist are shown in the following table of analyses :

COMPOSITION OF MILK.

\begin{tabular}{l|c|c|c|c|c|c}
\hline \multicolumn{1}{c|}{ In iuo Parts. } & Human. & Cow. & Goat. & Ass. & Sherp. & Mare. \\
\hline Water. & 88.00 & 86.87 & 87.54 & 91.57 & 82.27 & 88.80 \\
\hline Caseinogen. & 2.40 & 3.98 & 3.00 & 1.09 & 6.10 & 2.19 \\
\hline Lactalbumin. & 0.57 & 0.77 & 0.62 & 0.70 & 1.00 & 0.42 \\
\hline Fat. & 2.90 & 3.50 & 4.20 & 1.02 & 5.30 & 2.50 \\
\hline Lactose. & 5.87 & 4.00 & 4.00 & 5.50 & 4.20 & 5.50 \\
\hline Salts. & 0.16 & 0.17 & 0.56 & 0.42 & $\mathbf{I . 0 0}$ & 0.50 \\
\hline
\end{tabular}

Caseinogen is the chief proteid constituent of milk, and is held in solution by the presence of calcic phosphate. On the addition of acetic acid or of sodic chlorid up to the point of saturation, the caseinogen is precipitated as such, and may be collected by appropriate chemic methods. When taken into the stomach caseinogen is coagulated-that is, it is separated into casein or tyrein and a small quantity of a new soluble proteid. The ferment which induces this change is known as rennin. The presence of calcic phosphate is necessary for this coagulation.

The fat of milk is more or less solid at ordinary temperatures. It is a composition of olein, palmitin, and stearin, with a small quantity of butyrin and caproin. When milk is allowed to stand for some time the fat-globules rise to the surface and form a thick layer, known as cream. When 
subjected to the churning process, the fat globules run together and form a cohesive mass-the butter.

Lactose is the particular form of sugar characteristic of milk. It belongs to the saccharose group and has the following composition : $\mathrm{C}_{12} \mathrm{H}_{22}$ $\mathrm{O}_{11}$. In the presence of the bacillus acidi lactici the lactose is decomposed into lactic acid and carbon dioxid, the former of which will cause a coagulation of the caseinogen.

Mechanism of Secretion.-During the time of lactation the mammary gland exhibits periods of secretory activity which alternate with periods of rest. Coincidentally with these periods, certain histologic changes take place in the secreting structures of the gland. At the close of a period of active secretion each acinus presents the following features: the epithelial cells are short, cubic, nucleated, and border a relatively wide lumen in which is to be found a variable quantity of non-discharged milk. After the gland has rested for some time, active metabolism again begins. The epithelial cells grow and elongate; the nucleus divides into two or three new nuclei, and at the same time the cell becomes constricted; the inner portion is detached and is discharged into the lumen. Coincidentally with these changes oil-globules make their appearance in the cell protoplasm, some of which are discharged separately into the lumen, while others remain for a time associated with the detached cell. From these histologic changes it would appear that the caseinogen and the fat-globules are metabolic products of the cell protoplasm, and not derived directly from the blood. That lactose has a similar origin appears certain from the fact that it is formed independently of carbohydrate food. The water and inorganic salts are doubtless secreted by a mechanism similar to that of all other secreting glands.

\section{VASCULAR OR DUCTLESS GLANDS.}

\section{INTERNAL SECRETIONS.}

The metabolism of the body generally, as well as that of individual organs, has been shown to be related not only to the physiologic activity of such organs as the liver and pancreas, but also to the activity of the so-called vascular or ductless glands. The influence of the pancreas in regulating the production of glycogen by the liver, and the influence of the liver in the maintenance of the general metabolism through the pro- 
duction of glycogen and the formation of urea, are now established facts. That the vascular or ductless glands to an equal extent, though perhaps in a different way, assist in the maintenance of physiologic processes, appears certain from the results of animal experimentation. The explanation given, and generally accepted at the present time, for the influence of these glands is that they produce specific substances, which are poured into the blood or lymph and carried direct to the tissues, to the activities of which they appear to be essential ; for without these substances the nutrition of the tissues declines and in a short time a fatal termination ensues.

Inasmuch as these partly unknown substances are formed by cell activity and are poured into the interstices of the tissues, they have been termed " internal secretions." Though the term internal secretions is applicable to all substances which arise in consequence of tissue metabolism, and which, after being poured into the blood, influence in varying degrees and ways physiological processes, yet the term in this connection will be applied only to the secretions of the thyroid gland, hypophysis cerebri, and adrenal bodies.

Thyroid Gland.-The thyroid gland or body consists of two lobes situated on the lateral aspect of the upper part of the trachea. Each lobe is pyriform in shape, the base being directed downward and on a level with the fifth or sixth tracheal ring. The lobe is about $50 \mathrm{~mm}$. in length, $20 \mathrm{~mm}$. in breadth, and $25 \mathrm{~mm}$. in thickness. As a rule, the lobes are united by a narrow band or isthmus of the same tissue. In color the gland is reddish, and it is abundantly supplied with blood-vessels and lymphatics.

Microscopic examination shows that the thyroid consists of an enormous number of closed sacs or vesicles, variable in size, the largest not meas uring more than $0.1 \mathrm{~mm}$. in diameter. Each sac is composed of a thin homogeneous membrane lined by cuboid epithelium. The interior of the sac in adult life contains a transparent viscid fluid, containing albumin and termed "colloid" substance. Externally, the sacs are surrounded by a plexus of capillary blood-vessels and lymphatics. The individual sacs are united and supported by connective tissue, which forms, in addition, a covering for the entire gland.

Function of the Thyroid.-The knowledge at present possessed as to the function of the thyroid gland, especially in mammals, is the outcome of a study of the effects which follow its arrest of development in the child, its degeneration in the adult, its extirpation in the human being as well as 
in animals. The results, however, which follow its extirpation are not always uniform in all animals; sufficient reasons for which lack of uniformity can not always be assigned.

Cretinism, a condition characterized by a want of physical and mental development, is associated with, if not directly dependent on, a congenital absence or an arrested development of the thyroid, either at the time of birth or during the early years of childhood.

Myxedema, a condition of the skin in which there is a hyperplasia of the connective tissue, of an embryonic type, rich in mucin, is generally regarded as one of the effects of degenerative processes in the thyroid. Partly in consequence of this change in the skin the face becomes broader, swollen, and flattened, giving rise to a loss of expression. At the same time the mind becomes dull, clouded, even approximating the idiotic type. This supposed infiltration of the skin with mucin was termed myxedema by Ord, who at the same time associated it with a change in the structure of the thyroid as a result of which it became functionally useless.

Extirpation of the thyroid, for relief from symptoms due to grave pathologic changes, has been followed in human beings by symptoms similar to those of myxedema. To this condition the terms operative myxedema and cachexia strumipriva have been applied.

After the publication of the history of the myxedema which followed surgical removal of the thyroid, Schiff, in 1887 , repeated his earlier experiments on dogs, and found again that removal of the thyroid was speedily followed by tremors, convulsions, and death. Similar experiments were made by Horsley on monkeys, with results which resembled those characteristic of myxedema. Among the symptoms which developed within a few days after the removal of the gland may be mentioned loss of appetite; fibrillar contractions of muscles; tremors; spasms ; mucinoid degeneration of the skin, giving rise to puffiness of the eyelids and face and to a swollen condition of the abdomen; hebetude of mind, frequently terminating in idiocy ; fall of blood-pressure; dyspnea; albuminuria; atrophy of the tissues, followed by death of the animal in the course of from five to eight weeks. The complexus of symptoms observed in monkeys was divided by Horsley into three stages: vix., the neurotic, the mucinoid, and the atrophic. It is evident that the presence of the thyroid is essential to the normal activity of the tissues generally. As to the manner in which it exerts its favorable influence, there is some difference of opinion. The view that the gland removes from the blood certain toxic bodies, rendering them innocuo,us, and thus preserving the body from a species of auto-intoxication, is gradually yielding to the more probable view that the epithelinm is engaged to the 
secretion of a specific material, which finds its way into the blood or lymph and in some unknown way influences favorably tissue metabolism. This view of the function of the thyroid is supported by the fact that successful grafting of a portion of the thyroid beneath the skin or in the abdominal cavity will prevent the usual symptoms which follow thyroidectomy. The same result is obtained by the intravenous injection of thyroid juice or by the administration of the raw gland. It was shown by Murray that myxedematous patients could be benefited, and even cured, by feeding them with fresh thyroids or even with the dry extract.

The chemic features of the material secreted and obtained from the structures of the thyroid indicate that it is a complex proteid containing iodin, which, under the influence of various reagents, undergoes cleavage, giving rise to a non-proteid residue, which carries with it the iodin and phosphorus. The amount of iodin in the thyroid varies from 0.33 to I milligram for each gram of tissue. To this compound the term thyroiodin has been given. The administration of this compound produces effects similar to those which follow the therapeutic administration of the fresh thyroid itself : viz., a diminution of all myxedematous symptoms. In normal states of the body, thyroiodin influences very actively the general metabolism. It gives rise to a decomposition of fats and proteids and to a decline in body-weight. In large doses it may produce toxic symptoms : e. g., increased cardiac action, vertigo, and glycosuria.

The Hypophysis Cerebri.-This is a small body lodged in the sella turcica of the sphenoid bone. It consists of an anterior lobe, somewhat red in color, and a posterior lobe, yellowish-gray in color. The former is much the larger and partly embraces the latter. The anterior lobe is developed from an invagination of the epiblast of the mouth cavity, and consists of distinct gland tissue. The posterior lobe is an outgrowth from the brain, and is connected with the infundibulum by a short stalk. It has been suggested that the term infundibular body be reserved for the posterior lobe. This distinction appears to be desirable, inasmuch as in their origin and structure they are separate and distinct bodies.

Removal of the hypophysis cerebri, or the pituitary body, is always followed by a fatal result, preceded by symptoms not unlike those which follow removal of the thyroid: viz., anorexia, tremors, spasms, etc. Degeneration of the hypophysis has been found in connection with a hypertrophic condition of the bones of the face and extremities, to which the term acromegalia has been given.

Intravenous injection of an extract of the hypophysis increases the 
force of the heart-beat without any change in its frequency, and causes a rise of blood-pressure from a stimulation of the arterioles (Schäfer and Oliver). The material secreted by the hypophysis has not been isolated, hence its chemic features are unknown. After its formation it probably passes through a system of ducts into the cerebrospinal fluid, after which it influences the metabolism of the nervous and osseous tissues as well as the force of the heart muscle.

An extract of the hypophysis itself exerts no appreciable effect on the blood-pressure or on the rate of the heart-beat, nor does it influence the circulatory and respiratory organs (Howell). An extract of the infundibular body intravenously injected, however, gives rise to increased bloodpressure and to a slowing of the heart-beat.

Adrenal Bodies, or Suprarenal Capsules. - These are two flattened bodies, somewhat crescentic or triangular in shape, situated each upon the upper extremity of the corresponding kidney, and held in place by connective tissue. They measure about $40 \mathrm{~mm}$. in height, $30 \mathrm{~mm}$. in breadth, and from 6 to $8 \mathrm{~mm}$. in thickness. The weight of each is about $4 \mathrm{gm}$.

Function of the Adrenal Bodies.-It was observed by Addison that a profound disturbance of the nutrition, characterized by a bronze-like discoloration of the skin and mucous membranes of the mouth, extreme muscular weakness, and profound anemia, was associated with, if not dependent on, pathologic conditions of the suprarenal glands. In the progress of the disease the asthenia gradually increases, the heart becomes weak, the pulse small, soft, and feeble, indicating a general loss of tone of the muscular and vascular apparatus. Death ensues from paralysis of the respiratory muscles. The essential nature of the lesion which gives rise to these symptoms has not been determined.

Removal of these bodies from various animals is invariably and in a short time followed by death, preceded by some of the symptoms characteristic of Addison's disease. Their development, however, was more acute. From the fact that animals so promptly die from extirpation of these bodies, and the further fact that the blood of such animals is toxic to those the subjects of recent extirpation, but not to normal animals, the conclusion was drawn that the function of the adrenal bodies was to remove from the blood some toxic material the product of muscle metabolism. Its accumulation after extirpation gives rise to death through auto-intoxication.

On the supposition that the adrenals might secrete and pour into the blood a specific material which favorably influences general imetabolism, 
Schäfer and Oliver injected hypodermically glycerin and water extracts, and observed at once an increased activity of the heart-beats and of the respiratory movements. The effects, however, were only transitory. When these extracts are injected into the veins directly, there follows in a short time a cessation of the auricular contraction of the heart, though the ventricular contraction continues with an independent rhythm. If the vagi are cut previous to the injection or if the inhibition is removed by atropin, the rapidity and vigor of both auricles and ventricles are increased. Whether the inhibitory influence is removed or not, there is a marked increase in the blood-pressure, though it is greater in the former than in the latter instance. This is attributed to a direct stimulation and contraction of the musclefibers of the arterioles themselves, and not to vaso-motor influences, as it occurs also after division of the cord and destruction of the bulb. The contraction of the arterioles is quite general, as shown by plethysmographic studies of the limbs, spleen, kidney, etc. Applied locally to the mucous membranes, the adrenal extract produces contraction of the blood-vessels and pallor. The skeletal muscles are affected by the extract very much as they are by veratrin. The duration of a single contraction is very much prolonged, especially in the phase of relaxation or of decreasing energy.

It is evident from these experiments that the adrenal bodies are engaged in elaborating and pouring into the blood a specific material which stimulates to increased activity the muscle-fibers of the heart and arteries, and thus assists in maintaining the normal blood-pressure as well as the tonicity of the skeletal muscles. The active principle of this gland has been isolated by Abel and termed epinephrin.

\section{EXCRETION.}

The principal excrementitious fluids discharged from the body are the urine, perspiration, and bile; they hold in solution principles of waste which are generated during the activity of the nutritive process and are the ultimate forms to which the organic constituents are reduced in the body. They also contain inorganic salts.

The urinary apparatus consists of the kidneys, ureters, and bladder.

\section{KIDNEYS.}

The kidneys are the organs for the secretion of urine; they resemble a bean in shape, are from four to five inches in length, two in breadth, and weigh from four to six ounces. 
They are situated in the lumbar region, one on each side of the vertebral column behind the peritoneum, and extend from the eleventh rib to the crest

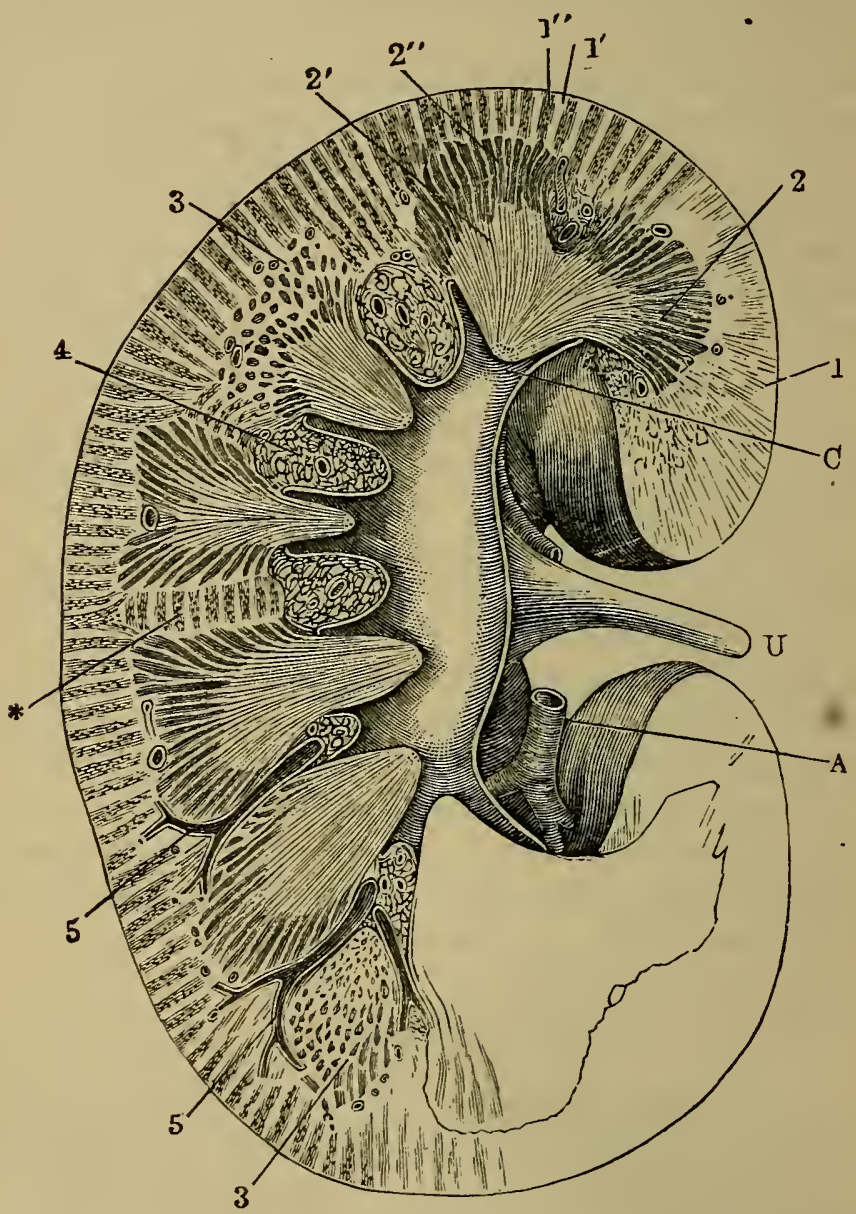

lig. ig.-Longitudinal Section through the Kidney, the Pelvis of the Kidiney, and a number of Renal Calyces.-(Tyson, after Henle.)

A. Branch of the renal artery. U. Ureter. C. Renal calyx. r. Cortex. $x^{\prime}$. Medullary rays. I". Labyrinth, or cortex proper. 2. Meduila. 2'. Papillary portion of medulla, or nedulla proper. $2^{\prime \prime}$. Border layer of the medulla. 3,3 . Transverse section through the axes of the tubules of the border layer. 4. Fat of the renal sinus. 5, 5. Arterial branches. *. Transversely coursing medulla rays.

of the ilium; the anterior surface is convex, the posterior surface concave, the latter presenting a deep notch, the hilus.

The kidney is surrounded by a thin, smooth membrane composed of 
white fibrous and yellow elastic tissue; though it is attached to the surface of the kidney by minute processes of connective tissue, it can be readily torn away. The substance of the kidney is dense, but friable.

Upon making a longitudinal section of the kidney it will be observed that the hilus extends into the interior of the organ and expands to form a cavity known as the sinus. This cavity is occupied by the upper, dilated portion of the ureter, the interior of which forms the pelvis. The ureter subdivides into several portions, which ultimately give origin to a number of smaller tubes, termed calyces, which receive the apices of the pyramids.

The parenchyma of the kidney consists of two portions-viż. :

1. An internal or medullary portion, consisting of a series of pyramids or cones, some twelve or fifteen in number. They present a distinctly striated appearance, a condition due to the straight direction of the tubules and blood-vessels.

2. An external or cortical portion, consisting of a delicate matrix containing an immense number of tubules having a markedly convoluted appearance. Throughout its structure are found numerous small ovoid bodies, termed Malpighian curpuscles.

The Uriniferous Tubules. - The kidney is a compound, tubular gland composed of microscopic tubules whose function it is to secrete from the blood those waste products which collectively constitute the urine. If the apex of each pyramid be examined with a lens, it will present a number of small orifices, which are the beginnings of the uriniferous tubules. From this point the tubules pass outward in a straight

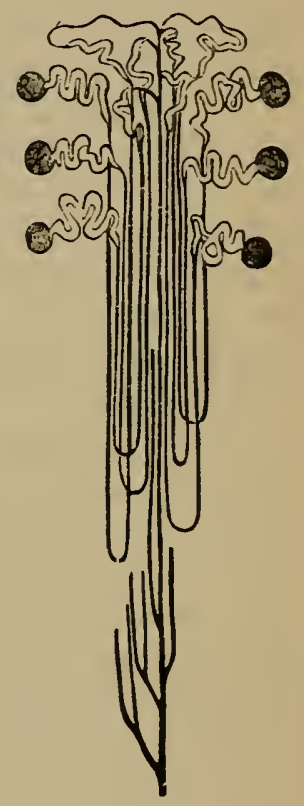

Fig. 20.-Diagramimatic EXPOSITION OF THE METHOD IN WHICH THE URINIFEROUS TUBES UNITE TO FORM PRIMITIVE CONES. - (T'son, after Ludwig.) but somewhat divergent manner toward the cortex, giving off at acute angles a number of branches (Fig. 20). From the apex to the base of the pyramids they are known as the tubules of Bellini. In the cortical portion of the kidney each tubule becomes enlarged and twisted, and after pursuing an extremely convoluted course, turns backward into the medullary portion for some distance, forming the descending limb of Henle's loop : 
it then turns upon itself, forming the ascending limb of the loop, reēnters the cortex, again expands, and finally terminates in a spheric enlargement known as Mïller's or Bozman's capsule. Within this capsule is contained a small tuft of blood-vessels, constituting the glomerulus, or Malpighian corpuscle.

Structure of the Tubules.-Each tubule consists of a basement membrane lined by epithelial cells throughout its entire extent. The tubule and its contained epithelium vary in shape and size in different parts of its course. The termination of the convoluted tube consists of a little sac or capsule, which is ovoid in shape and measures about $\frac{1}{2} 00$ of an inch. This capsule is lined by a layer of flattened epithelial cells, which is also reflected over the surface of the glomerulus. During the periods of secretory activity the blood-vessels of the glomerulus become filled with blood, so that the cavity of the sac is almost obliterated; after secretory activity the blood-vessels contract and the sac-cavity becomes enlarged. In that portion of the tubule lying between the capsule and Henle's loop the epithelial cells are cuboid in shape; in Henle's loop they are flattened, while in the remainder of the tubule they are cuboid and columnar.

Blood-vessels of the Kidney.-The renal artery is of large size and enters the organ at the hilum; it divides into several large branches, which penetrate the substance of the kidney between the pyramids, at the base of which they form an anastomosing plexus, which completely surrounds them. From this plexus vessels follow the straight tubes toward the apex, while others, entering the cortical portion, divide into small twigs, which enter the Malpighian body and form a mass of convoluted vessels, the glomerulus. After circulating through the Malpighian tuft, the blood is gathered together by two or three small veins, which again subdivide and form a fine capillary plexus, which envelops the convoluted tubules; from this plexus the veins converge to form the emulgent vein, which empties into the vena cava.

The nerves of the kidney follow the course of the blood-vessels and are derived from the renal plexus.

The ureter is a membranous tube, situated behind the peritoneum, about the diameter of a goose-quill, eighteen inches in length, and extends from the pelvis of the kidney to the base of the bladder, which it perforates in an oblique direction. It is composed of three coats : fibrous, muscular, and mucous.

The bladder is a reservoir for the temporary reception of the urine 
prior to its expulsion from the body; when fully distended it is ovoid in shape, and holds about one pint. It is composed of four coats : serous, muscular (the fibers of which are arranged longitudinally and circularly), areolur, and mucous. The orifice of the bladder is controlled by the sphincter vesica, a muscular band about $1 / 2$ of an inch in width.

As soon as the urine is formed it passes through the tubuli uriniferi into the pelvis and thence through the ureters into the bladder, which it enters at an irregular rate. Shortly after a meal, after the ingestion of large quantities of fluid, and after exercise, the urine flows into the bladder quite rapidly, while it is reduced to a few drops during the intervals of digestion. It is prevented from regurgitating into the ureters by the oblique direction they take between the mucous and muscular coats.

Nervous Mechanism of Urination. - When the urine has passed into the bladder, it is there retained by the sphincter vesicæ muscle, kept in a state of chronic contraction by the action of a nerve center in the lumbar region of the spinal cord. This center can be inhibited and the sphincter relaxed, either reflexly, by impressions coming through sensory nerves from the mucous membrane of the bladder, or directly, by a voluntary impulse descending the spinal cord. When the desire to urinate is experienced, impressions made upon the vesical sensory nerves are carried to the centers governing the sfhincter and detrusor urine muscles and to the brain. If now the act of urination is to take place, a voluntary impulse originating in the brain passes down the spinal cord and still further inbibits the sphincter vesicre center, with the effect of relaxing the muscle and of stimulating the center governing the detrusor muscle, with the effect of contracting the muscle and expelling the urine. If the act is to be suppressed, voluntary impulses inhibit the detrusor center and possibly stimulate the splincter center.

The genitospinal center controlling these movements is situated in that portion of the spinal cord corresponding to the origin of the third, fourth, and fifth sacral nerves.

\section{URINE.}

Normal urine is of a pale yellow or amber color, perfectly transparent, with an aromatic odor, an acid reaction, a specific gravity of 1020 , and a temperature when first discharged of $100^{\circ} \mathrm{F}$.

The color varies considerably in health, from a pale yellow to a brown lue, owing to the presence of the coioring-matter, urobilin or wrochrome. 
The transparency is diminished by the presence of mucus, the calcium and magnesium phosphates, and the mixed urates.

The reaction of the urine is acid, owing to the presence of acid phosphate of sodium. The degree of acidity, however, varies at different periods of the day. Urine passed in the morning is strongly acid, while that passed during and after digestion, especially if the food is largely vegetable in character, is either neutral or alkaline.

The specific gravity varies from ror 5 to 1025 .

The quantity of urine excreted in twenty-four hours is between forty and fifty fluidounces, but ranges above and below this standard.

The odor is characteristic, and caused by the presence of taurylic and phenylic acids, but is influenced by vegetable foods and other substances eliminated by the kidneys.

\section{COMPOSITION OF URINE.}

Water, . . . . . . . . . . . . . 967.0

Urea, .......... 14.230

Other nitrogenized crystalline bodies, uric acid, principally in the form of alkaline urates,

Creatin, creatinin, xanthin, hypoxanthin,

Hippuric acid, leucin, tyrosin, taurin, cystin, all in small amounts, and not constant,

Mucus and pigment,

Salts

Inorganic: principally sodium and potassium sulphates, phosphates, and chlorids, with magnesium and calcium phosphates, traces of silicates and chlorids,

Organic: lactates, hippurates, acetates, formates, which appear only occasionally,

Sugar

Gases (nitrogen and carbonic acid principally).

\section{,}

- a trace

$\overline{1,000.000}$

The average quantity of the principal constituents excreted in twentyfour hours is as follows :

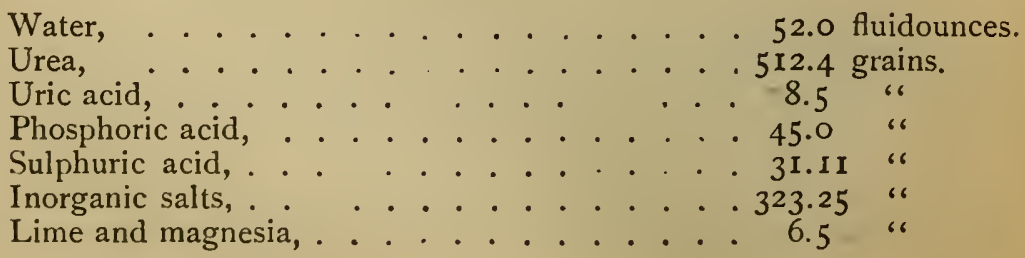


To determine the amount of solid matters in any given amount of urine, multiply the last two figures of the specific gravity by the coefficient of Haeser, 2.33-e. $g$., in $\mathrm{I}, 000$ grains of urine having a specific gravity I022, there are contained $22 \times 2.33=5 \mathrm{r} .26$ grains of solid matter.

Organic Constituents of Urine.-Urea is one of the most important of the organic constituents of the urine, and is present to the extent of from 2.5 to 3.2 per cent. Urea is a colorless, neutral substance, crystallizing in four-sided prisms terminated by oblique surfaces. When crystallization is caused to take place rapidly, the crystals take the form of long, silky needles. Urea is soluble in water and alcohol; when subjected to prolonged boiling, it is decomposed, giving rise to carbonate of ammonia. In the alkaline fermentation of urine, urea takes up two molecules of water with the production of carbonate of ammonia.

The average amount of urea excreted daily has been estimated at about 500 grains. As urea is one of the principal products of the breaking up of the albuminous compounds within the body, it is quite evident that the quantity produced and eliminated in twenty-four hours will-be increased by any increase in the amount of albuminous food consumed, or by a rapid destruction of albuminous tissues, as is observed in various pathologic states, inanition, febrile conditions, fevers, etc. A farinaceous or vegetable diet will diminish the urea production nearly one half.

Muscular exercise when the nutrition of the body is in a state of equilibrium does not seem to increase the quantity of urea.

Seat of Urea Formation.-As to the seat of urea formation, little is positively known. It is quite certain that it preëxists in the blood and is merely excreted by the kidneys. It is not produced in muscles, as even after prolonged exercise hardly a trace of urea is to be found in them. Experimental and pathologic facts point to the liver as the probable organ engaged in urea formation. Acute yellow atrophy of the liver, suppurative diseases of the liver, diminish almost entirely the production of urea.

Uric acid is also a constant ingredient of the urine and is closely allied to urea. It is a nitrogenized substance, carrying out of the body a large quantity of nitrogen. The amount eliminated daily varies from five to ten grains. Uric acid is a colorless crystal belonging to the rhombic system. It is insoluble in water, and if eliminated in excessive amounts, it is deposited as a "brick-red" sediment in the urine. It is doubtful if uric acid exists in a free state, being combined for the most part with sodium and potassium bases forming urates. It is to be regarded as one of the termi- 
nal products of the disassimilation of albuminous compounds, and is probably produced in the liver.

Hippuric acid is found very generally in urine, though it is present only in small amounts. It is increased by a diet of asparagus, cranberries, plums, and by the administration of benzoic and cinnamic acids. It is probably formed in the kidney.

Kreatinin resembles the kreatin derived from muscles. It is a colorless crystal, belonging to the rhombic system. Its origin is unknown, though it is largely increased in amount by albuminous food. About fifteen grains are excreted daily.

Xanthin, sarkin, oxaluric acid, and allantoin are also constituents of urine. They are nitrogenized compounds and are also terminal products of albuminous compounds.

Urobilin, the coloring-matter of the urine, is a derivative of the bile pigments. It is particularly abundant in febrile conditions, giving to the urine its reddish-yellow color.

Inorganic Constituents of Urine.-Earthy Phosphate. Phosphoric acid in combination with magnesium and calcium is excreted daily to the extent of from fifteen to thirty grains. The phosphates are insoluble in water, but are held in solution in the urine by its acid ingredients, alkalinity of the urine being attended with a copious precipitation of the phosphates. Mental work increases the amount of phosphoric acid excreted, a condition caused by increased metabolism of the nervous tissue.

Sulphuric acid in combination with sodium and potassium constitutes the sulphates, of which about thirty grains are excreted daily. Sulphuric acid results largely from the decomposition of albuminous food and from increased destruction of animal tissues.

The gases of urine are carbonic acid and nitrogen.

Mechanism of Urinary Secretion.-As the kidney anatomically presents an apparatus for filtration (the Malpighian bodies) and an apparatus for secretion (the epithelial cells of the urinary tubules), it might be inferred that the elimination of the constituents of the urine is accomplished by the twofold process of filtration and secretion; that the water and highly diffusible inorganic salts simply pass by diffusion through the walls of the blood-vessels of the glomerulus into the capsule of Müller, while the urea and remaining organic constituents are removed by true secretory action of the renal epithelium. Modern experimentation supports this view of renal action. 
The secretion of urine is, therefore, partly physical and partly vital.

The filtration of urinary constituents from the glomerulus into Müller's capsule depends largely upon the blood-pressure and the rapidity of blood flow in the renal artery and glomerulus. Among the influences which increase the pressure and velocity may be mentioned increased frequency and force of the heart's action, contraction of the capillary vessels of the body generally, dilatation of the renal artery, and increase in the volume of the blood.

The reverse conditions lower the blood-pressure and diminish the secretion of urine.

The fact that organic matters are eliminated by the secretory activity of the renal epithelium seems to be well established by modern experiments. These substances, removed from the blood in the secondary capillary plexus of blood-vessels, by a true selective action of the epithelium, are dissolved and washed toward the pelves by the liquid coming from the capsules.

The blood-supply to the kidney is regulated by the nervous system. If the renal nerves be divided, the renal artery dilates and a copious flow of urine takes place. If the peripheral ends of the same nerves be stimulated, the artery contracts and the urinary flow ceases. The same is true of the splanchnic nerves, through which the vaso-motor nerves coming from the medulla oblongata and spinal cord pass to the renal plexus.

\section{LIVER.}

The liver is a highly vascular, conglomerate gland, appended to the alimentary canal. It is the largest gland in the body, weighing about four and one half pounds; it is situated in the right hypochondriac region, and is retained in position by five ligaments, four of which are formed by duplicatures of the peritoneal investment.

The proper coat of the liver is a thin but firm fibrous membrane, closely adherent to the surface of the organ, which it penetrates at the transverse fissure, and follows the vessels in their ramifications through its substance, constituting Glisson's capsule.

Structure of the Liver.-The liver is made up of a large number of small bodies (the lobuies), rounded or ovoid in shape, measuring $\frac{1}{25}$ of an inch in diameter, separated by a space in which are situated blood-vessels, nerves, bepatic ducts, and lymphatics. 
The lobules are composed of cells, which, when examined microscopically, exhibit a rounded or polygonal shape, and measure, on the average, $\frac{1}{1000}$ of an inch in diameter; they possess one, and sometimes two, nuclei ; they also contain globules of fat, pigment matter, and animal starch. The cells constitute the secreting structure of the liver, and are the true hepatic cells.

The blood-vessels which enter the liver are:

I. The portal vein, made up of the gastric, splenic, and superior and inferior mescnteric veins.

2. The hepatic artery, a branch of the celiac axis.

Both the portal vein and the hepatic artery are invested by a sheath of areolar tissue.

The vessels which leave the liver are the hepatic veins, originating in its interior, collecting the blood distributed by the portal vein and hepatic artery, and conducting it to the ascending vena cava.

Distribution of Vessels.--The portal vein and the hepatic artery, upon entering the liver, penetrate its substance, divide into smaller and smaller branches, occupy the spaces between the lobules, completely surrounding and limiting them, and constitute the interlobular vessels. The hepatic artery, in its course, gives off branches to the walls of the portal vein and Glisson's capsule, and finally empties into the small branches of the portal vein in the interlobular spaces.

The interlobular vessels form a rich plexus around the lobules, from which branches pass to neighboring lobules and enter their substance, where they form a very fine network of capillary vessels, ramifying over the hepatic cells, in which the various functions of the liver are performed. The blood is then collected by small veins, converging toward the center of the lobule, to form the intralobular vein, which runs through its long axis and empties into the sublobular vein. The hepatic veins are formed by the union of the sublobular veins, and carry the blood to the ascending vena cava; their walls are thin and adherent to the substance of the hepatic tissue.

The hepatic ducts or bile capillaries originate within the lobules, in a very fine plexus lying between the hepatic cells; whether the smallest vessels have distinct membranous walls, or whether they originate in the spaces between the cells by open orifices, has not been satisfactorily determined.

The bile-channels empty into the interlobular ducts, which measure about $\frac{1}{2000}$ of an inch in diameter, and are composed of a thin, homogeneous membrane lined by flattened epithelial cells. 
As the interlobular bile-ducts unite to form large trunks, they receive an external coat of fibrous tissue, which strengthens their walls; they finally unite to form one large duct (the hepatic duct), which joins the cystic duct; the union of the two forms the ductus communis choledochus, which is about three inches in length, the size of a goose-quill, and opens into the duodenum.

The gall-bladder is a pear-shaped sac, about four inches in length, situated in a fussa on the under surface of the liver. It is a reservoir for the bile, and is capable of holding about one ounce and a half of fluid. It is composed of three coats :

I. Serous, a reflection of the peritoneum.

2. Fibrous and muscular.

3. Mucous.

Functions of the Liver.-The liver is a complex organ having a variety of relations to the general processes of the body. While its physiologic actions are not yet wholly understood, it may be said that it-

I. Secretes bile.

2. Forms glycogen.

3. Assists in the formation of urea and allied products.

4. Modifies the composition of the blood as it passes through it.

The Secretion of Bile. - The characteristic constituents of the bile do not preëxist in the blood, but are formed in the interior of the liver cells of materials derived from the venous and arterial blood. The hepatic cells, absorbing these materials, elaborate them into bile-elements, and in so doing undergo histologic changes similar to those exhibited by other secretory glands. The bile once formed, it passes into the mouths of the bile capillaries, near the periphery of the lobules. Under the influence of the vis a tergo of the new-formed bile it flows from the smaller into the larger bile-ducts, and finally empties into the intestine, or is regurgitated into the gall-bladder, where it is stored up until it is required for the digestive process in the small intestine. The study of the secretion of bile by means of biliary fistulæ reveals the fact that the secretion is continuous and not intermittent; that the hepatic cells are constantly pouring bile into the ducts, which convey it into the gall-bladder. As this fluid is required only during intestinal digestion, it is only then that the walls of the gall-bladder contract and discharge it into the intestine.

The flow of bile from the liver cells into the gall-bladder is accomplished by the inspiratory movements of the diaphragm, and by the contraction of the muscle-fibers of the biliary ducts, as well as the vis a tergo of new- 
formed bile. Any obstacle to the outflow of bile into the intestine leaps to an accumulation within the bile-ducts. The pressure within the ducts increasing beyond that of the blood within the capillaries, a reabsorption of biliary matters by the lymphatics takes place, giving rise to the phenomena of jaundice.

The bile is both a secretion and an excretion; it contains new constituents, which are formed only in the substance of the liver, and are destined to play an important part ultimately in nutrition; it contains also waste ingredients, which are discharged into the intestinal canal and eliminated from the body.

Glycogenic Function.--In addition to the preceding function, Bernard, in 1848 , demonstrated the fact that the liver, during life, normally produces a sugar-forming substance, analogous in its chemic composition to starch, which he terms glycogen; also that, when the liver is removed from the body, and its blood-vessels are thoroughly washed out, after a few hours sugar again makes its appearance in abundance.

It can be shown to exist in the blood of the hepatic vein as well as in a decoction of the liver substance by means of either Trommer's or Fehling's test, even when the blood of the portal vein does not contain a trace of sugar.

Origin and Destination of Glycogen.-Glycogen appears to be formed de novo in the liver cells, from materials derived from the food, whether the diet be animal or vegetable, though a larger percentage is formed when the animal is fed on starchy and saccharin than when fed on animal food. The glucose, which is one of the products of digestion, is absorbed by the blood-vessels and carried directly into the liver; as it does not appear in the urine, as it would if injected at once into the general circulation, it is probable that it is detained in the liver, dehydrated, and stored up as glycogen. The change is shown by the following formula:

$$
\begin{aligned}
& \mathrm{C}_{6} \mathrm{H}_{12} \mathrm{O}_{6}-\mathrm{H}_{2} \mathrm{O}=\mathrm{C}_{6} \mathrm{H}_{10} \mathrm{O}_{5} \text {. } \\
& \text { Glucose. Water. Glycogen. }
\end{aligned}
$$

The glycogen thus formed is stored up in the hepatic cells for the future requirements of the system. When it is carried from the liver, it is again transformed into glaiose by the agency of a ferment. Glycogen does not undergo oxidation in the blood; this process takes place in the tissues, particularly in the muscles, where it generates heat and contributes to the development of muscular force. 
Glycogen, when obtained from the liver, is an amorphous, starch-like substance, of a white color, tasteless and colorless, and soluble in water; by boiling with dilute acids, or subjected to the action of an animal ferment, it is easily converted into dextrose. When an excess of sugar is generated by the liver, dextrose can be found not only in the blood of the hepatic vein, but also in other portions of the body ; under these circumstances it is eliminated by the kidneys, appearing in the urine, constituting the condition of glycosuria.

Formation of Urea.-The liver is now regarded by many physiologists as the principal organ concerned in urea formation. The liver normally contains a certain amount of urea; and if blood be passed through the excised liver of an animal which has been in full digestion when kilied, a large amount of urea is obtained. The clinical evidence proves that in destructive diseases of the liver substance there is at once a falling-off in urea elimination. Various drugs which stimulate liver action increase the urea in the urine.

Elaboration of Blood.-Besides the capability of secreting bile, the liver possesses the property of so acting upon and modifying the chemic composition of the products of digestion as they traverse its substance that they are readily assimilated by the blood, and are transformed into materials capable of being converted into the elements of the blood and into solid tissues.

The albuminous principles particularly require the modifying influence of the liver; for if they are removed from the portal vein and introduced into the jugular vein, they are at once removed from the blood by the action of the kidneys.

The blood of the hepatic vein differs from the blood of the portal vein in being richer in blood-corpuscles, both red and white; its plasma is more dense, containing a smaller percentage of water and a greater amount of solid constituents, but no fibrin; its serum contains less albumin, fats, and salts, but its sugar is increased.

Influence of the Nervous System.-The nervous system directly controls the functional activity of the liver, and more especially its glycogenic function. It was discovered by Bernard that puncture of the medulla oblongata is followed by so enormous a production of sugar that it is at once excreted by the kidneys, giving rise to diabetic or saccharine urine. This part of the medulla is, however, the vaso-motor center for the bloodvessels of the liver. Destruction of this center, or injury to the vaso-motor nerves emanating from it in any part of their course, is followed at once by dilatation of the hepatic blood-vessels, slowing of the blood-current, a pro- 
found disturbance of the normal relation existing between the blood and liver-cells, and a production of sugar. Many of the hepatic vaso-motor nerves may be traced down the cord as far as the lumbar region, while others leave the cord high up in the neck and enter the cervical ganglia of the sympathetic, and so reach the liver. Injury to the sympathetic ganglia is often followed by diabetes. Peripheral stimulation of various nerves, e. $g$., sciatic, pneumogastric, depressor nerve, - as well as the direct action of many drugs, impair or depress the hepatic vaso-motor center and so give rise to diabetes.

\section{SKIN.}

The skin, the external investment of the body, is a most complex and important structure, serving -

I. As a protective covering.

2. As an organ for tactile sensibility.

3. As an organ for the elimination of excrementitious matters.

The amount of skin investing the body of a man of average size is about twenty feet, and varies in thickness, in different situations, from $\frac{1}{8}$ to $\frac{1}{10}$ of an inch.

The skin consists of two principal layers - viz., a deeper portion, the corium, and a superficial portion, the epidermis.

The corium, or cutis vera, may be subdivided into a reticulated and a papillary layer. The former is composed of white fibrous tissue, nonstriated muscle-fibers, and elastic tissue, interwoven in every direction, forming an areolar network, in the meshes of which are deposited masses of fat, and a structureless, amorphous matter ; the latter is formed mainly of club-shaped elevations or projections of the amorphous matter, constituting the papilla; they are most abundant and well developed upon the palms of the hands and upon the soles of the feet; they average $\frac{1}{100}$ of an inch in length, and may be simple or compound; they are well supplied with nerves, blood-vessels, and lymphatics.

The epidermis, or scarf skin, is an extravascular structure, a product of the true skin, and is composed of several layers of cells. It may be divided into two layers: the rete mucosum, or the Malpighian layer, and the horny or corneous.

The former is closely adherent to the papillary layer of the true skin, 
and is composed of large, nucleated cells, the lowest layer of which, the "prickle cells," contains pigment-granules, which give to the skin its varying tints in different individuals and in different races of men; the more superficial cells are large, colorless, and semi-transparent. The latter, the corneous layer, is composed of flattened cells, which, from their exposure to the atmosphere, are hard and horny in texture; it varies in thickness from $\frac{1}{8}$ of an inch on the palms of the hands and soles of the feet to $\frac{1}{900}$ of an inch in the external auditory canal.

\section{APPENDAGES OF THE SKIN.}

Hairs are found in almost all portions of the body, and can be divided into -

I. Long, soft hairs, on the head.

2. Short, stiff hairs, along the edges of the eyelids and nostrils.

3. Soft, downy hairs on the general cutaneous surface.

They consist of a root and a shaft. The latter is oval in shape and about $\frac{1}{400}$ of an inch in diameter; it consists of fibrous tissue, covered externally by a layer of imbricated cells, and internally by cells containing granular and pigment material.

The root of the hair is embedded in the hair-follicle, formed by a tubular depression of the skin, extending nearly through to the subcutaneous tissue ; its walls are formed by the layers of the corium, covered by epidermic cells. At the bottom of the follicle is a papillary projection of amorphous matter, corresponding to a papilla of the true skin, containing blood-vessels and nerves, upon which the hair-root rests. The investments of the hair-roots are formed of epithelial cells, constituting the internal and external rootsheaths.

The hair protects the head from the heat of the sun and from the cold, retains the heat of the body, prevents the entrance of foreign matter into the lungs, nose, ears, etc. The color is due to pigment matter. In old age the hair becomes more or less whitened.

The sebaceous glands, embedded in the true skin, are simple and compound racemose glands, opening, by a common excretory duct, upon the surface of the epidermis or into the hair-follicle. They are found in all portions of the body, most abundantly in the face, and are formed by a delicate, structureless membrane, lined by flattened polyhedral cells. The sebaceous glands secrete a peculiar oily matter (the sebum), by which the 
skin is lubricated and the hairs are softened; it is quite abundant in the region of the nose and forehead, which often presents a greasy, glistening appearance; it consists of water, mineral salts, fatty globules, and epithelial cells.

The vernix caseosa, which frequently covers the surface of the fetus at birth, consists of the residue of the sebaceous matter, containing epithelial cells and fatty matters; it seems to keep the skin soft and supple, and guards it from the effects of the long-continued action of the amniotic water.

The sudoriparous glands excrete the sweat. They consist of a mass or coil of a tubular gland duct, situated in the derma and in the subcutaneous tissue, average $\frac{1}{75}$ of an inch in diameter, and are surrounded by a rich plexus of capillary blood-vessels. From this coil the duct passes in a straight direction up through the skin to the epidermis, where it makes a few spiral turns and opens obliquely upon the surface. The sweat-glands consist of a delicate homogeneous membrane lined by epithelial cells, whose function is to extract from the blood the elements existing in the perspiration.

The glands are very abundant all over the cutaneous surface-as many at 3528 to the square inch, according to Erasmus Wilson.

The perspiration is an excrementitious fluid, clear, colorless, almost odorless, slightly acid in reaction, with a specific gravity of 1003 to 1004 .

The total quantity of perspiration excreted daily has been estimated at about two pounds, though the amount varies with the nature of the food and drink, exercise, external temperature, season, etc.

The elimination of the sweat is not intermittent, but continuous; it takes place so gradually that as fast as it is formed it passes off by evaporation as insensible perspiration. Under exposure to great heat and exercise the evaporation is not sufficiently rapid, and it appears as sensible perspiration.

\section{COMPOSITION OF SWEAT.}

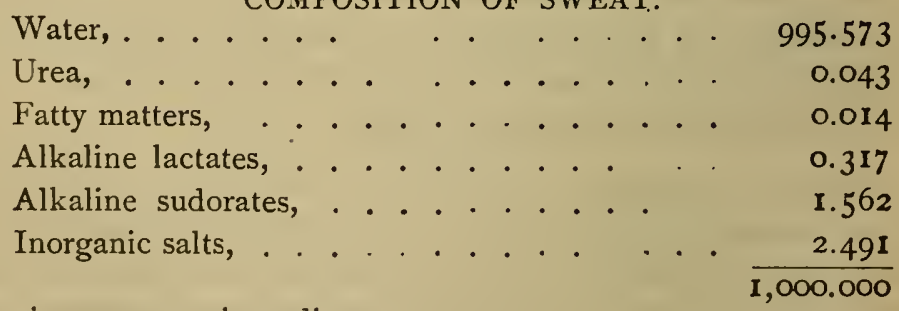

Urea is a constant ingredient.

Carbonic acid is also exhaled from the skin, the amount being about $\frac{1}{2} 00$ of that from the lungs. 
Perspiration regulates the temperature and removes waste matters from the blood ; it is so important that if elimination be prevented, death occurs in a short time.

Influence of the Nervous System.-The secretion of sweat is regulated by the nervous system. Here, as in the secreting glands, the fluid is formed from material in the lymph-spaces surrounding the gland. Two sets of nerves are concerned-viz., vaso-motor, regulating the blood-supply ; and secretory, stimulating the activities of the gland cells. Generally the two conditions, increased blood flow and increased glandular action, coexist. At times profuse clammy perspiration occurs, with diminished blood flow.

The dominating sweat-center is located in the medulla, though subordinate centers are present in the cord. The secretory fibers reach the perspiratory glands of the head and face through the cervical sympathetic; of the arms, through the thoracic sympathetic, ulnar, and radial nerves; of the leg, through the abdominal sympathetic and sciatic nerves.

The sweat-center is excited to action by mental emotions, increased temperature of blood circulating in the medulla and cord, increased venosity of blood, many drugs, rise of external temperature, exercise, etc.

\section{CEREBRO-SPINAL AXIS.}

The cerebro-spinal axis consists of the spinal cord, medulla oblongata, pons Varolii, cerebellum, and cerebrum, exclusive of the spinal and cranial nerves. It is contained within the cavities of the cranium and spinal column, and surrounded by three membranes,- - the dura mater, arachnoid, and pia mater,-which protect it from injury and supply it with blood-vessels.

\section{MEMBRANES.}

The dura mater, the outermost of the three, is a tough membrane, composed of white fibrous tissue arranged in bundles, which interlace in every direction. In the cranial cavity it lines the inner surface of the bones, and is attached to the edge of the foramen magnum ; it sends processes inward, forming the falx cerebri, falx cerebelli, and tentorium cerebelli, supporting and protecting parts of the brain. In the spinal canal it loosely invests the cord, and is separated from the walls of the canal by areolar tissue. 
The arachnoid, the middle membrane, is a delicate serous structure which envelops the brain and cord, forming the visceral layer, and is then reflected to the inner surface of the dura mater, forming the parietal layer. Between the two layers there is a small quantity of fluid, which prevents friction by lubricating the two surfaces.

The pia mater, the innermost of the three, composed of areolar tissue and blood-vessels, covers the entire surface of the brain and cord, to which it is closely adherent, dipping down between the convolutions and fissures. It is exceedingly vascular, sending small blood-vessels some distance into the brain and cord.

The cerebro-spinal fluid occupies the subarachnoid space and the general ventricular cavities of the brain, which communicate by an opening (the foramen of Magendie) in the pia mater, at the lower portion of the fourth ventricle. This fluid is clear, transparent, alkaline, possesses a salty taste, and has a low specific gravity; it is composed largely of water, and there are traces of albumin, glucose, and mineral salts. It is secreted by the pia mater; the quantity is estimated at from two to four fluidounces.

The function of the cerebro-spinal fluid is to protect the brain and cord by preventing concussion from without; as it is easily displaced into the spinal canal, it prevents undue pressure and insufficiency of blood to the brain.

\section{SPINAL CORD.}

The spinal cord varies from sixteen to eighteen inches in length; it is $1 / 2$ of an inch in thickness, weighs $I 1 / 2$ ounces, and extends from the atlas to the second lumbar vertebra, terminating in the filum terminale. It is cylindric in shape, and presents an enlargement in the lower cervical and lower dorsal regions, corresponding to the origin of those nerves which are distributed to the upper and lower extremities. The cord is divided into two lateral halves by the anterior and posterior fissures. It is composed of both while or fibrous and gray or vesicular matter, the former occupying the exterior of the cord, the latter the interior, where it is arranged in the form of two crescents, one in each lateral half, united by the central mass, the gray commissure; the white matter being united in front by the white commissure.

Structure of the Gray Matter.-The gray matter is arranged in the form of two crescents, united by a commissural band, forming a figure 
resembling the letter $\mathrm{H}$. Each crescent presents an anterior and a posterior horn. The center of the commissure presents a canal which extends from the fourth ventricle downward to the filum terminale. The anterior horn is short and broad and does not extend to the surface. The posterior horn is narrow and elongated and extends quite to the surface. It is covered and capped by the substantia gelatinosa. The gray matter consists primarily of a framework of fine connective tissue, supporting blood-vessels, lymphatics, medullated and non-medullated nerve-fibers, and groups of nerve-cells.

The nerve-cells are arranged in groups, which extend for some distance throughout the cord, forming columns more or-less continuous. The first group is situated in the anterior horn, the cells of which are large, multipolar, and connected with the anterior roots of the spinal nerves. The second group is situated in the posterior horn, the cells of which are spindleshaped, and from their relation to the posterior roots are supposed to be sensory in function. The third group is situated in the lateral aspect of the gray matter, and is quite separate and distinct, except in the lumbar and cervical enlargements, where it blends with those of the anterior horn. A fourth group is situated at the inner

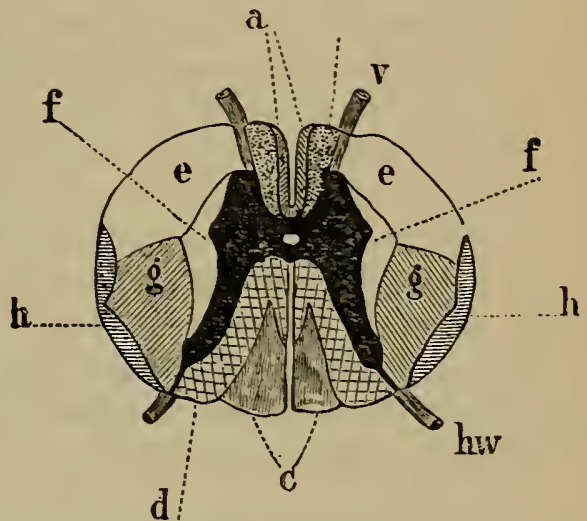

Fig. 2I.-Scheme of the Conducting PATH iN THE SPINAL CoRd at THE Third Dorsal Nerve.-(Landois)

The black pairt is the gray matter. v. Anterior, hw, posterior, root. a. Direct, and $\mathrm{g}, \mathrm{g}$, crossed, pyramidal tracts. b. Anterior column, ground bundle. c. Goll's column. d. Postero-external column. e, e, and f, f. Mixed lateral paths. h, h. Direct cerebellar tracts. base of the posterior horn; it begins about the seventh or eighth cervical nerve and extends downward to the second or third lumbar, being nost prominent in the dorsal region. This column is known as Clark's vesicular column.

Structure of the White Matter.-The white matter surrounding each lateral half of the cord is made up of nerve-fibers, some of which are continuations of the nerves which enter the cord, while others are derived from different sources. It is subdivided into- 
I. An anterior column, comprising that portion between the anterior roots and the anterior fissure, which is again subdivided into two parts :

(a) An inner portion, bordering the anterior median fissure, the direct pyramidal tract, or column of Türck; it contains motor fibers which do not decussate, and which extend as far down as the middle of the dorsal region.

(b) An outer portion, surrounding the anterior cornua, known as the anterior root zone, composed of short, longitudinal fibers which serve to connect different segments of the spinal cord.

2. A lateral column, the portion between the anterior and posterior roots, which is divisible into-

(a) The crossed pyramidal tract, occupying the posterior portion of the lateral column, and containing all those fibers of the motor tract which have decussated at the medulla oblongata; it is composed of longitudinally running fibers, which are connected with the multipolar nerve-cells of the anterior cornua.

(b) The direct cerebellar tract, situated upon the surface of the lateral column, consisting of longitudinal fibers which terminate in the cerebellum; it first appears in the lumbar region, and increases in thickness as it passes upward.

(c) The anterior tract, lying just posterior to the anterior cornua.

3. A posterior column, the portion included between the posterior roots and the posterior fissure, also divisible into two portions :

(a) An inner portion, the postero-internal column, or the column of Goll, bordering the posterior median fissure, and

(b) An external portion, the postero-external column, the column of Burdach, lying just behind the posterior roots.

The two portions of the posterior column are composed of long and short commissural fibers, which connect different segments of the spinal cord.

\section{SPINAL NERVES.}

Origin.-The spinal nerves are thirty-one in number on each side of the spinal cord, and arise by two roots, an anterior and a posterior, from the anterior and posterior aspect of the cord, respectively. The posterior roots present, near their emergence from the cord, a small ganglionic enlargement. Outside of the spinal canal the two roots unite to form a main trunk, which is ultimately distributed to the skin, muscles, and viscera.

For the functions of the roots of the spinal nerves, see page 77 . 


\section{COURSE OF THE ANTERIOR AND POSTERIOR ROOTS.}

The anterior roots pass through the anterior columns, horizontally, in straight and distinct bundles, and enter the anterior cornua, where they diverge in four directions :

I. Many become connected with the prolongations of the multipolar nervecells.

2. Others leave the gray matter, pass through the anterior white commissure, and enter the anterior columns of the opposite side.

3. A considerable number enter the lateral columns of the same side, through which they pass to the medulla oblongata, where they decussate and finally terminate in the corfus striatum of the opposite side.

4. Others traverse the gray matter horizontally, and come into relation with the cells of the intermediary lateral column.

The posterior roots enter the posterior horns of the gray matter-

1. Through the substantia gelatinosa.

2. Through the posterior columns.

Of the former some bend upward and downward, and become connected with the anterior cornua; others pass through the posterior commissure to the opposite side ; of the latter, fibers pass into the gray matter to the posterior vesicular columns, passing obliquely through the posterior white columns upward and downward for some distance, and enter the gray matter at different heights.

Decussation of Motor and Sensory Fibers.-The motor fibers, which conduct volitional impulses from the brain outward to the anterior cornua, arise in the motor centers of the cerebrum ; they then pass downward through the corona radiata, the internal capsule, the inferior portions of the crura cerebri, the pons Varolii, to the medulla oblongata, where the motor tract of each side divides into two portions, viz. :

1. The larger, containing ninety-one to ninety-seven per cent. of the fibers, which decussates at the lower border of the medulla and passes down in the lateral column of the opposite side, and constitutes the crossed pyramidal tract.

2. The smaller, containing three to nine per cent. of the fibers, does not decussate, but passes down the anterior column of the same side, and constitutes the direct pyramidal tract, or the column of Türck.

Some of the motor fibers of these two tracts, after entering the anterior cornua of the gray matter, become connected with the large multipolar 
nerve-cells, while others pass directly into the anterior roots. Through this decussation each half of the brain governs the muscular movements of the opposite side of the body.

The sensory fibers, which convey to the cord and brain the impression made upon the periphery, pass into the cord through the posterior roots of spinal nerves; they then diverge and enter the gray matter at differen levels, and at once decussate, passing to the opposite side of the gray matter. The sensory tract passes upward, through the cord, the medulla, pons Varolii, the superior portion of the cruri cerebri, the posterior third of the internal capsule, to the sensory perceptive center, located in the hippocampus major and uncinate convolution (Ferrier). Through this decussation each half of the brain governs the sensibility of the opposite half of the body.

Properties of the Spinal Cord.-Irritation applied directly to the anterolateral white columns produces muscular movements, but no pain; they are, therefore, excitable, but insensible.

The surface of the posterior columns is not sensitive to direct irritation, except near the origin of the posterior roots. The sensibility is due, however, not to its own proper fibers, but to the fibers of the posterior root, which traverse it.

Division of the anterolateral columns abolishes all power of voluntary movement in the lower extremities.

Division of the posterior column impairs the power of muscular coördination, such as is witnessed in locomotor ataxia.

The gray matter is probably both insensible and inexcitable under the influence of direct stimulation.

A transverse section of one lateral half of the cord produces-

I. On the same side, paralysis of voluntary motion, a relative or absolute elevation of temperature, and an increased flow of blood in the paralyzed parts; hyperesthesia, for the sense of contact, tickling ,pain, and temperature.

2. On the opposite side, complete anesthesia as regards contact, tickling, and temperature in the parts corresponding to those which are paralyzed in the opposite side, with a complete preservation of voluntary power and of the muscular sense.

A vertical section through the middle of the gray matter results in the loss of sensation on both sides of the body below the section, but no loss of voluntary power. 


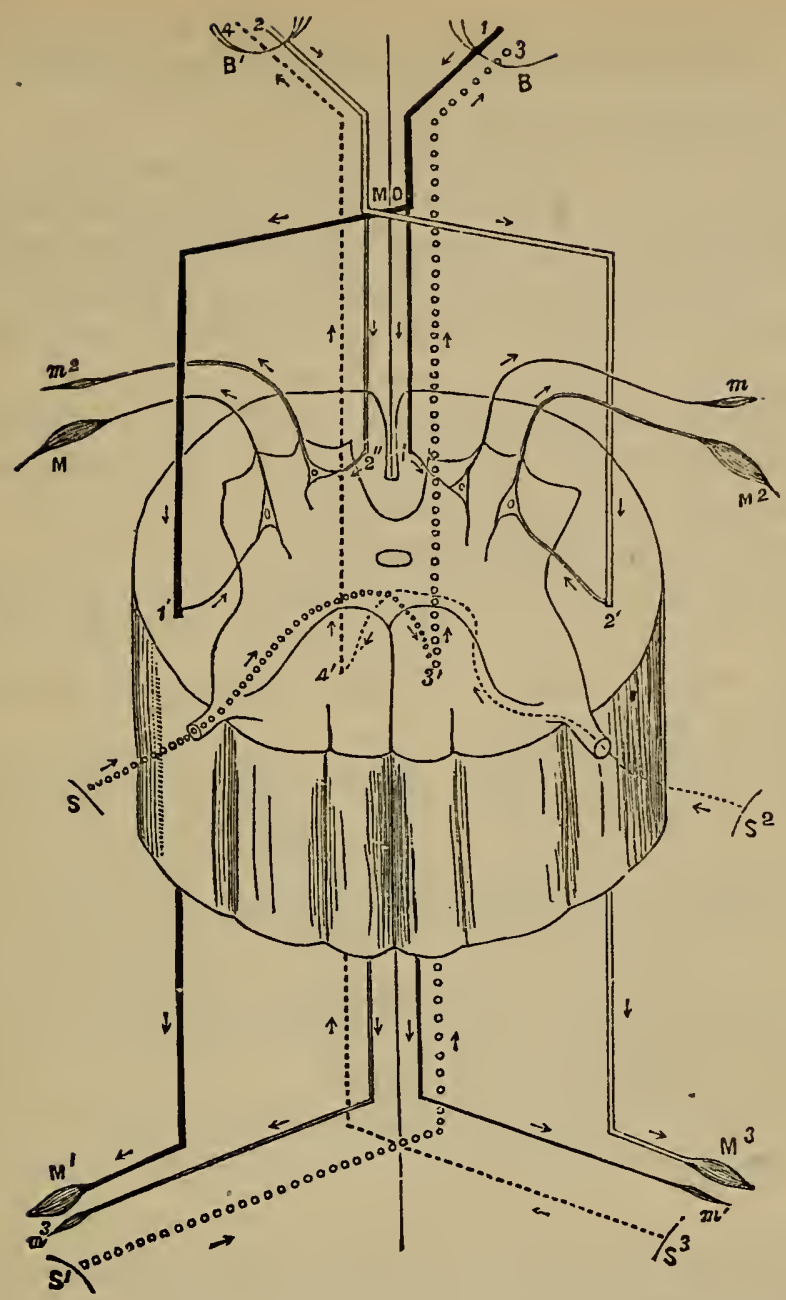

Fig, 22.-Diagrai showing the Course, through the Spinal Cord, of the Motor and Sensory Nerve-Fibers.

$B$ and $B^{\prime}$ represent the right and left hemispheres of the brain, from which the motor fibers take their origin, and in which the sensory fibers terminate. The motor tract from the right side, I, passes down through the crus, through the pons to the medulla oblongata, where it divides into two portions: (I) The larger portion, ninety-seven per cent., crosses over to the opposite side of the cord and passes down through the lateral column. It gives off fibers at different levels, which pass into the gray matter and become connected with the muscles, M, through the inultipolar cells. (2) The smaller portion, three per cent., does not cross over, but descends on the same side of the cord in the anterior column and supplies the muscles, $m$. The same is true for the motor tract for the left hemisphere.

The sensory fibers from the left side of the body enter the gray matter through the posterior roots. They then cross over at once to the opposite side of the cord and ascend to the hemisphere, partly in the gray matter, partly in the posterior column. The same is true for the sensory nerves of the right side of the body. 


\section{FUNCTIONS OF THE SPINAL CORD.}

\section{As an Independent Nerve Center.}

The spinal cord, in virtue of its contained nerve-cells, is capable of transforming afferent nerve impulses arriving through the afferent nerves into efferent impulses, which are reflected outward through efferent nerves to muscles, producing motion; to glands, exciting secretion ; to bloodvessels, changing their caliber. All such actions taking place independent of either sensation or volition are termed reflex actions. The mechanism involved in every reflex action consists of a sentient surface, an afferent nerve, an emissive center, an efferent nerve, and a responsive organ, muscle, gland, or blood-vessel (see p. 8I).

The reflex excitability of the cord may be-

I. Increased by disease of the lateral columns, by the administration of strychnin, and, in frogs, by a separation of cord from the brain, the latter apparently exerting an inhibitory influence over the former and depressing its reflex activity.

2. Inhibited by destructive lesion of the cord-e. $g$. , locomotor ataxia, atrophy of the anterior cornua-the administration of various drugs, and, in the frog, by irritation of certain regions of the brain. When the cerebrum alone is removed and the optic lobes are stimulated, the time elapsing between the application of an irritant to a sensory surface and the resulting movement will be considerably prolonged, the optic lobes (Setschenow's center) apparently generating impulses which, descending the cord, retard its reflex movement.

All movements taking place through the nervous system are of this reflex character, and may be divided into excito-motor, sensori-motor, and ideomotor.

Classification of Reflex Movements (Küss).-They may be divided into four groups, according to the route through which the centripetal and centrifugal impulses pass :

I. 'Those normal reflex acts (e.g., deglutition, coughing, sneezing, walking, etc.) and pathologic reflex acts (e.g., tetanus, vomiting, epilepsy) which take place both centripetally and centrifugally through spinal nerves.

2. Reflex acts which take place in a centripetal direction through a cerebro-spinal sensory nerve, and in a centrifugal direction through a sympathetic motor nerve, usually a vaso-motor nerve-e. g., the normal reflex acts, which give rise to most of the secretions, pallor of the skin and blushing, certain movements of the iris, certain modifications in the beat 
of the'heart ; the pathologic reflexes, which, on account of the difficulty in explaining their production, are termed metastatic-e.g., ophthalmia, coryza, orchitis, which depend on a reflex hyperemia; amaurosis, paralysis, paraplegia, etc., due to a reflex anemia.

3. Reflex movements in which the centripetal impulse passes through a sympathetic nerve, and the centrifugal through a cerebro-spinal nerve; most of these phenomena are pathologic-e. $g$., convulsions from intestinal irritation produced by the presence of worms, eclampsia, hysteria, etc.

4. Reflex actions in which both the centripetal and centrifugal impulses pass through filaments of the sympathetic nervous system-e. $g$., those obscure reflex actions which preside over the secretions of the intestinal fluids, which unite the phenomena of the generative organs, the dilatation of the pupils from intestinal irritation (worms), and many pathologic phenomena.

Laws of Reflex Action (Pflïger).

1. Law of Unilaterality. - If a feeble irritation be applied to one or more sensory nerves, movement takes place usually on one side only, and that the same side as the irritation.

2. Laze of Symmetry. - If the irritation becomes sufficiently intense, motor reaction is manifested, in addition, in corresponding muscles of the opposite side of the body.

3. Law of Intensity.-Reflex movements are usually more intense on the side of the irritation; at times the movements of the opposite side equal them in intensity, but they are usually less pronounced.

4. Law of Radiation. - If the excitation still continues to increase, it is propagated upward, and motor reaction takes place through centrifugal nerves coming from segments of the cord higher up.

5. Law of Generalization.-When the irritation becomes very intense, it is propagated in the medulla oblongata; motor reaction then becomes general, and it is propagated up and down the cord, so that all the muscles of the body are thrown into action, the medulla oblongata acting as a focus whence radiate all reflex movements.

\section{Special Reflex Movements.}

There are a number of reflex movements taking place through the spinal cord, a study of which enables the physician to determine the condition of its different segments. They may be divided into-

I. Skin or superficial, and

2. Tendon or deep reflexes. 
The skin reflexes are induced by irritation of the skin and mucous membranes-e.g., pricking, pinching, scratching, etc. The following are the principal skin reflexes :

I. Plantar reflex, consisting of contraction of the muscles of the foot, induced by stimulation of the sole of the foot; it involves the integrity of the reflex arc through the lower end of the cord.

2. Gluteal reflex, consisting of contraction of the glutei muscles when the skin over the buttock is stimulated; it takes place through the segments giving origin to the fourth and fifth lumbar nerves.

3. Cremasteric reflex, consisting of a contraction of the cremaster muscle and a retraction of the testicle toward the abdominal ring when the skin on the inner side of the thigh is stimulated; it depends upon the integrity of the segments giving origin to the first and second lumbar nerves. 4. Abdominal reflex, consisting of a contraction of the abdominal muscles when the skin upon the side of the abdomen is gently scratched; its production requires the integrity of the spinal segments from the eighth to the twelfth.

5. Epigastric reflex, consisting of a slight muscular contraction in the neighborhood of the epigastrium when the skin between the fourth and sixth ribs is stimulated; it requires the integrity of the cord between the fourth and seventh dorsal nerves.

6. The scapular reflex consists of a contraction of the scapular muscles when the skin between the scapulæ is stimulated; it depends upon the integrity of the cord between the fifth cervical and third dorsal nerves.

The superficial reflexes, though variable, are generally present in health. They are increased or exaggerated when the gray matter of the cord is abnormally excited, as in tetanus, strychnin-poisoning, and disease of the lateral columns, leading to arrest of their normal functions. The tendon or deep reflexes are also of great value in diagnosing the condition of the spinal segments. They are induced by a sharp blow upon a tendon. The following are the principal forms:

I. Patellar reflex, or knee-jerk, consisting of a contraction of the extensor muscles of the thigh when the ligamentum patella is struck between the patella and tibia. This reflex is best observed when the legs are freely hanging over the edge of a table. The patellar reflex is generally present in health, being absent in only two per cent.; it is greally exaggerated in lateral sclerosis and in descending degeneration of the cord; it is absent in locomotor ataxia and in atrophic lesions of the anterior gray cornuæ.

2. Ankle-jerk or Reflex. - If the extensor muscles of the leg be placed 
upon the stretch and the tendo Achillis be sharply struck, a quick extension of the foot will take place.

3. Ankle-clonus. - This consists of a series of rhythmic reflex contractions of the gastrocnemius muscle, varying in frequency from six to ten a second. To elicit this reflex, pressure is made upon the sole so as suddenly and energetically to flex the foot at the ankle, thus putting the tendo Achillis upon the stretch. The rhythmic movements thus produced continue as long as the tension is maintained. Ankle-clonus is never present in health, but is very marked in lateral sclerosis of the cord.

The toe reflex, peroneal reflex, and zurist reflex are also present in sclerosis of the lateral columns and in the late rigidity of hemiplegia.

Special Nerve Centers in Spinal Cord. - Throughout the spinal cord there are a number of special nerve centers, capable of being excited reflexly and of producing complex coördinated movements. Though for the most part independent in action, they are subject to the controlling influences of the medulla and brain.

I. Ciliospinal center, situated in the cord between the lower cervical and the third dorsal vertebra. It is connected with the dilatation of the pupil through fibers which emerge in this region and enter the cervical sympathetic. Stimulation of the cord in this locality causes dilatation of the pupil on the same side; destruction of the cord is followed by contraction of the pupil.

2. Genitospinal center, situated in the lower part of the cord. This is a complex center, and comprises a series of subordinate centers for the control of the muscular movements involved in the acts of defecation, micturition, and ejaculation of semen, and of the movements of the uterus during parturition, etc.

3. Vaso-motor centers, giving origin to both vaso-constrictor and vasodilator fibers, which are distributed throughout the cord. Though acting reflexly, they are under the dominating influence of the center in the medulla.

4. Sweat-centers are also present in various parts of the cord.

\section{As a Conductor.}

The lateral columns, particularly the posteriqr portions, the "pyramidal tracts," and the columns of Türck, are the channels through which pass the voluntary motor impulses from the brain to the large multipolar nervecells, in the anterior cornua of gray matter, and through them become 
connected with the anterior roots which transmit the motor stimuli to the muscles.

The anterior columns, especially the portion surrounding the anterior cornua, the "anterior radicular zones," are composed of short, longitudinal commissural fibers, which serve to connect different segments of the spinal cord, a condition required for the coördination of muscular movements.

The posterior columns are composed of short and long commissural fibers which connect different segments of the cord. They are insensible to direct irritation, but aid in the coördination of muscular movements in walking, standing, running, etc. Degeneration of the posterior columns gives rise to the lack of muscular coördination observed in locomotor ataxia.

The gray matter, and especially that portion immediately surrounding the central canal, transmits the sensory nerve-fibers from the posterior roots up to the brain. Decussation of the sensory fibers takes place throughout the whole length of the gray matter.

The multipolar cells of the anterior cornua are connected with the generation and transmission of motor impulses outward; are centers for reflex movements; are the trophic centers for the motor nerves and muscle-fibers to which they are distributed. The anterior roots give passage to the vaso-constrictor and vaso-dilator fibers, which exert an influence upon the caliber of the blood-vessels. Complete destruction of the anterior horns is followed by paralysis of motion, degeneration of the anterior roots, atrophy of muscles and bones, and abolition of reflex movements.

\section{Paralysis from Injuries of the Spinal Cord.}

Seat of Lesion.-If it be in the lower part of the sacral canal, there is paralysis of the compressor urethræ, accelerator urinæ, and sphincter ani muscles; no paralysis of the muscles of the leg.

At the Upper Limit of the Sacral Region.-Paralysis of the muscles of the bladder, rectum, and anus; loss of sensation and motion in the muscles of the legs, except those supplied by the anterior crural and obturatorviz., psoas iliacus, Sartorius, pectineus, adductores longus, magnus, and brevis, obturator, vasti externus and internus, etc.

At the Upper Limit of the Lumbar Region.-Sensation and motion paralyzed in both legs; loss of power over the rectum and bladder; paralysis of the muscular walls of the abdomen, interfering with expiratory movements. 
At the Lower Portion of the Cervical Region.-Paralysis of the legs, etc., as in the foregoing; in addition, paralysis of all the intercostal muscles and consequent interference with respiratory movements ; paralysis of muscles of the upper extremities, except those of the shoulders.

Above the Middle of the Cervical Region. - In addition to the preceding, difficulty of deglutition and vocalization, contraction of the pupils, paraly. sis of the diaphragm, scalene muscles, intercostals, and many of the accessory respiratory muscles; death resulting immediately from arrest of respiratory movements.

\section{CRANIAL NERVES.}

The cranial nerves come off from the base of the brain, pass through the foramina in the walls of the cranium, and are distributed to the skin, muscles, and organs of sense in the face and head.

According to the classification of Soemmering, there are twelve pairs of nerves, enumerating them from before backward, as follows-viz.:

First pair, or olfactory.

Second pair, or optic.

Third pair, or motor oculi communis.

Fourth pair, or patheticus (trochlearis).

Fifth pair, or trifacial (trigeminus). Sixth pair, or abducens.
Seventh pair, or facial (portio dura). Eighth pair, or auditory (portio mollis).

Ninth pair, or glosso-pharyngeal.

Tenth pair, or pneumogastric.

Twelfth pair, or hypoglossal.

The cranial nerves may also be classified physiologically, according to their function, into three groups :

I. Nerves of special sense.

2. Nerves of motion.

3. Nerves of general sensibility.

First Pair. Olfactory.

Apparent Origin.-From the inferior and internal portion of the anterior lobes of the cerebrum by three roots-viz., an external white root, which passes across the fissure of Sylvius to the middle lobe of the cerebrum; an internal white root, from the most posterior part of the anterior lobe; a gray root, from the gray matter in the posterior and inner portion of the inferior surface of the anterior lobe.

Deep Origin,--Not satisfactorily determined. 
Distribution.-The olfactory nerve, formed by the union of the three roots, passes forward along the under surface of the anterior lobe to the ethmoid bone, where it expands into the olfactory bulb. This bulb contains ganglionic cells and is grayish in color and soft in consistency; it gives off from its under surface from fifteen to twenty nerve filaments, the true olfactory nerves, which pass through the cribriform plate of the ethmoid bone and are distributed to the Schneiderian mucous membrane. This membrane extends from the cribriform plate of the ethmoid bone downward, about one inch.

Properties. - The olfactory nerves give rise to neither motor nor sensory phenomena when stimulated. They carry simply the special impressions of odorous substances. Destruction or injury of the olfactory bulbs is attended by a loss of the sense of smell.

Function.-Governs the sense of smell. Conducts the impressions which give rise to odorous sensations.

\section{Second Pair. Optic.}

Apparent Origin.-From the anterior portion of the optic commissure.

Deep Origin.-The origins and connections of the optic tract are very complex. The immediate origins are bands of fibers from the thalamus opticus and anterior corpora quadrigemina. The corpora geniculata are interposed ganglia. The ultimate roots are traced-

I. By a broad band of fibers- "the optic radiation of Gratiolet"--to the psycho-optic centers in the occipital lobes.

2. To the gyrus hippocampi and sphenoid lobes.

3. Through the corpus callosum to the motor areas of the opposite cerebral hemispheres.

4. To the frontal region by "Meynert's commissure."

5. To the spinal cord.

6. To the corpora geniculata, pulvinar, and anterior corpula geniculata by ganglionic roots.

Distribution.- The two roots unite to form a flattened band, the optic tract, which winds around the crus cerebri to decussate with the nerve of the opposite side, forming the optic chiasm. The decussation of fibers is not complete; some of the fibers of the left optic tract going to the outer half of the eye of the same side and to the inner half of the eye of the opposite side ; the same holds true for the right optic tract. 
The optic nerves proper arise from the commissure, pass forward through the optic foramina, and are finally distributed in the retina.

Properties.-They are insensible to ordinary impressions, and convey only the special impressions of light. Division of one of the nerves is attended by complete blindness in the eye of the corresponding side.

Hemiopia and Hemianopsia.-O Owing to the decussation of the fibers in the optic chiasm, division of the optic tract produces loss of sight in the outer half of the eye of the same side, and in the inner half of the eye of the opposite side, the blind part being separated from the normal part by a vertical line. The term hemiopia is applied to the loss of function or paralysis of the one half of the retina; hemianopsia is applied to the blindness in the field of vision. If, for example, the right optic tract be divided, there will be hemiopia in the outer half of the right eye and inner half of the left eye, thus causing left lateral hemianopsia, and as the two halves are affected which correspond in normal vision, the condition is known as homonymous hemianopsia. Lesion of the anterior part of the optic chiasm causes blindness in the inner half of the two eyes.

Functions.-Governs the sense of sight. Receives and conveys to the brain the luminous impressions which give rise to the sensation of sight.

The reflex movements of the iris are called forth by the optic nerve. When an excess of light falls upon the retina, the impression is carried back to the tubercula quadrigemina, where it is transformed into a motor impulse, which then passes outward through the motor oculi nerve to the contractile fibers of the iris and diminishes the size of the pupil. The absence of light is followed by a dilatation of the pupil.

\section{Third Pair. Motor Oculi Communis.}

Apparent Origin.--From the inner surface of the crura cerebri.

Deep Origin.--By three sets of filaments coming from the oculomotorius nucleus, which lies under the aqueduct of Sylvius; these three groups of filaments are destined for the innervation of the muscles of the eyeball, the sphincter pupillæ, and the ciliary muscle. By filaments coming from the lenticular nucleus, corpora quadrigemina, optic thalamus; these filaments converge to form a main trunk, which winds around the crus cerebri, in front of the pons Varolii.

Distribution.-The nerve then passes forward, and enters the orbit through the sphenoid fissure, where it divides into a superior branch dis- 
tributed to the superior rectus and levator palpebra muscles; an inferior branch, sending branches to the internal and inferior recti and the inferior oblique muscles; filaments also pass into the ciliary or ophthalmic ganglion ; from this ganglion the ciliary nerves arise, which enter the eyeball and are distributed to the circular fibers of the iris and the ciliary muscle. The third nerve also receives filaments from the cavernous plexus of the sympathetic and from the fifth nerve.

Properties.- Irritation of the root of the nerve produces contraction of the pupil, internal strabismus, and muscular movements of the eye, but no pain. Division of the nerve is followed by ptosis (falling of the upper eyelid); external strabismus, due to the unopposed action of the external rectus muscle; paralysis of the accommodation of the eye; dilatation of the pupil from paralysis of the circular fibers of the iris and ciliary muscle ; and inability to rotate the eye, slight protrusion, and double vision. The images are crossed; that of the paralyzed eye is a little above that of the sound, and its upper end inclined toward it.

Function.-Governs movements of the eyeball by animating all the muscles except the external rectus and superior oblique, influences the movements of the iris, elevates the upper lid, influences the accommodation of the eye for distances. Can be called into action by (I) voluntary stimuli, (2) by reflex action through irritation of the optic nerve.

\section{Fourth Pair. Patheticus.}

Apparent Origin.-From the superior peduncles of the cerebellum.

Deep Origin.-By fibers terminating in the corpora quadrigemina, lenticular nucleus, valve of Vieussens, and substance of the cerebellar peduncles; some filaments pass over the median line and decussate with fibers of the opposite side.

Distribution.-The nerve enters the orbital cavity through the sphenoid fissure, and is distributed to the superior oblique muscle; in its course it receives filaments from the ophthalmic branch of the fifth pair and the sympathetic.

Properties. - When the nerve is irritated, muscular movements are produced in the superior oblique muscle, and the pupil of the eye is turned downward and outward. Division or paralysis lessens the movements and rotation of the globe downward and outward. The diplopia consequent upon this paralysis is homonymous, one image appearing above the other. 
The image of the paralyzed eye is below, its upper end inclined toward that of the sound eye.

Function.-Governs the movements of the eyeball produced by the action of the superior oblique muscles.

\section{Sixth Pair.* Abducens. Motor Oculi Externus.}

Apparent Origin.-From the groove between the anterior pyramidal body and the pons Varolii, where it arises by two roots.

Deep Origin.-From the gray matter of the medulla oblongata.

Distribution.- The nerve then passes into the orbit through the sphenoid fissure, and is distributed to the external rectus muscle. Receives filaments from the cervical portion of the sympathetic, through the carotid plexus, and spheno-palatine ganglion.

Properties.-When irritated, the external rectus muscle is thrown into convulsive movements and the eyeball is turned outward. When divided or paralyzed, this muscle is paralyzed, motion of the eyeball outward past the median line is impossible, and the homonymous diplopia increases as the object is moved outward past this line. The images are upon the same plane and parallel. Internal strabismus results because of the unopposed action of the internal rectus.

Function.-To turn the eyeball outward.

\section{Fifth Pair. Trifacial. Trigeminal.}

Apparent Origin.-By two roots from the side of the pons Varolii.

Deep Origin.-The deep origin of the two roots is the upper part of the floor and anterior wall of the fourth ventricle, by three bundles of filiments, one of which anastomoses with the auditory nerve; another passes to the lateral tract of the medulla ; while a third, grayish in color, goes to the restiform bodies, and may be traced to the point of the calamus scriptorius.

Filaments of origin have been traced to the "trigeminal sensory nucleus," located on a level with the point of exit of the nerve, and to the posterior gray horns of the cord, as low down as the middle of the neck.

\footnotetext{
* The sixth nerve is considered in connection with the third and fourth nerves, since they together constitute the motor apparatus by which the ocular muscles are excited to action.
} 
Distribution.-The large root of the nerve passes obliquely upward and forward to the ganglion of Gasser, which receives filaments of communication from the carotid plexus of the sympathetic. It then divides into three branches :

I. Ophthalmic branch, which receives communicating filaments from the sympathetic, and sends sensitive fibers to all the motor nerves of the eyeball. It is distributed to the ciliary ganglion, to the lacrymal gland, sac, and caruncle, to the conjunctiva, integument of the upper eyelid, forehead, side of head and nose, anterior portion of the scalp, ciliary muscle, and iris.

2. Superior maxillary branch, sends branches to the sphenopalatine ganglion, integument of the temple and lower eyelid, side of the forehead nose, cheek, upper lip, teeth of the upper jaw, and alveolar processes.

3. Inferior maxillary branch, which, after receiving in its course filaments from the small root and from the facial, is distributed to the submaxillary ganglion, the parotid and sublingual glands, external auditory meatus, mucous membrane of the mouth, anterior two thirds of the tongue (lingual branch), gums, arches of the palate, teeth of the lower jaw, and integument of the lower part of the face, and to the muscles of mastication.

The small root passes forward beneath the ganglion of Gasser, through the foramen ovale, and joins the inferior maxillary division of the large root, which then divides into an anterior and a posterior branch, the former of which is distributed to the muscles of mastication-viz., temporal, masseter, and internal and external pterygoid muscles.

Properties.- It is the most acutely sensitive nerve in the body, and endows all the parts to which it is distributed with general sensibility.

Irritation of the large root, or of any of its branches, will give rise to marked evidence of pain; the various forms of neuralgia of the head and face being occasioned by compression, disease, or exposure of some of its terminal branches.

Division of the large root within the cranium is followed at once by a complete abolition of all sensibility in the head and face, but is not attended by any loss of motion. The integument, the mucous membranes, and the eye may be lacerated, cut, or bruised, without the animal exhibiting any evidence of pain. At the same time the lacrymal secretion is diminished, the pupil becomes contracted, the eyeball is protruded, and the sensibility of the tongue is abolished.

The reflex movements of deglutition are also somewhat impaired, the 
impression of the food being unable to reach and excite the nerve center in the medulla oblongata.

Galvanization of the small root produces movements of the muscles of mastication ; section of the root causes paralysis of these muscles, and the jaw is drawn to the opposite side by the action of the opposing muscles.

Influences of the Special Senses.-After division of the large root within the cranium, a disturbance in the nutrition of the special senses sooner or later manifests itself.

Sight.-In the course of twenty-four hours the eye becomes very vascular and inflamed, the cornea becomes opaque and ulcerates, the humors are discharged, and the eye is totally destroyed.

Sinell. - The nasal mucous membrane swells up, becomes fungous, and is liable to bleed on the slightest irritation. The mucous is increased in amount, so as to obstruct the nasal passages; the sense of smell is finally abolished.

Hearing.-At times the hearing is impaired from disorders of nutrition in the middle ear and external auditory meatus.

Alteration in the nutrition of the special senses is not marked if the section is made posterior to the ganglion of Gasser and to the anastomosing filaments of the sympathetic, which join the nerves at this point; but if the ganglion be divided, these effects are very noticeable, owing to the section of the sympathetic filaments.

Function.-Gives sensibility to all parts of the head and face to which it is distributed; through the small root, endows the masticatory muscles with motion; through fibers from the sympathetic, governs the nutrition of the special senses.

\section{Seventh Pair. Portio Dura. Facial Nerve.}

Apparent Origin.-From the groove between the olivary and restiform bodies at the lateral portion of the medulla oblongata and below the margin of the pons Varolii.

Deep Origin.-From a nucleus of large cells in the floor of the fourth ventricle, below the nucleus of origin of the sixth pair, with which it is connected. Some filaments are traceable to the lenticular nucleus of the opposite side. Some of the fibers cross the median line and decussate. It is intimately associated with the nerve of Wrisberg at its origin.

Distribution.-From its origin the facial nerve passes into the internal auditory meatus, and then, in company with the nerve of Wrisberg, enters 
the aqueduct of Fallopius. The filaments of the nerve of Wrisberg are supplied with a ganglion, of a reddish color, having nerve-cells. These filaments unite with those of the root of the facial to form a common trunk, which emerges at the stylomastoid foramen.

In the aqueduct the facial gives off the following branches-viz. :

1. Large petrosal nerve, which passes forward to the spheno-palatine, or Meckel's ganglion, and through this to the levator palati and azygos uvulæ muscles, which receive motor influence from this source.

2. Small petrosal nerve, passing to the otic ganglion and thence to the tensor tympani muscle, endowing it with motion.

3. Tympanic branch, giving motion to the stapedius muscle.

4. Chorda tympani nerve, which, after entering the posterior part of the tympanic cavity, passes forward between the malleus and incus, through the Glaserian fissure, and joins the lingual branch of the fifth nerve. It is then distributed to the mucous membrane of the anterior two thirds of the tongue and the submaxillary glands.

After emerging from the stylomastoid foramen, the facial nerve sends branches to the muscles of the ear, the occipitofrontalis, the digastric, the palatoglossi, and palatopharyngei ; after which it passes through the parotid gland and divides into the temporofacial and cervicofacial branches, which are distributed to the superficial muscles of the face-viz., occipitofrontalis, corrugator supercilii, orbicularis palpebrarum, levator labii superioris et alæque nasi, buccinator, levator anguli oris, orbicularis oris, zygomatici, depressor anguli oris, platysma myoides, etc.

Properties.-Undoubtedly a motor nerve at its origin, but in its course receives sensitive filaments from the fifth pair and the pneumogastric.

Irritation of the nerve, after its emergence from the stylomastoid foramen, produces convulsive movements in all the superficial muscles of the face. Division of the nerve at this point causes paralysis of these muscles on the side of the section, constituting facial paralysis, the phenomena of which are a relaxed and immobile condition of the same side of the face; the eyelids remain open, from paralysis of the orbicularis palpebrarum; the act of winking is abolished; the angle of the mouth droops, and saliva constantly drains away; the face is drawn over to the second side; the face becomes distorted upon talking or laughing; mastication is interfered with, the food accumulating between the gums and cheek, from paralysis of the buccinator muscle; fluids escape from the mouth in drinking; articulation is impaired, the labial sounds being imperfectly pronounced. 
Properties of the Branches given off in the Aquaduct of liullopius.-I the large petrosal, when irritated, throws the levator palati and azygos uvulæ muscles into contraction. Paralysis of this nerve, from deep-seated lesions, produces a deviation of the uvula to the sound side, a drooping of the palate, and an inability to elevate it.

The small petrosal influences hearing by animating the tensor tympani muscles; when paralyzed, there occurs partial deafness and an increased sensibility to sonorous impressions.

The tympanitic branch animates the stapedius muscle and influences audition.

The chorda tympani influences the circulation and the secretion of saliva in the submaxillary glands, and governs the sense of taste in the anterior two thirds of the tongue. Galvanization of the chorda tympani dilates the blood-vessels, increases the quantity and rapidity of the stream of blood, and increases the secretion of saliva. Division of the nerve is followed by contraction of the vessels, an arrest of the secretion, and a diminution of the sense of taste on the same side.

Function.-The facial is the nerve of expression, and coördinates the muscles employed to delineate the various emotions, influences the sense of taste, deglutition, movements of the uvula and soft palate, the tension of the membrana tympani, and the secretions of the submaxillary and parotid glands. Indirectly influences smell, hearing, and vision.

\section{Eighth Pair. Portio Mollis. Auditory Nerve.}

Apparent Origin.-From the upper and lateral portion of the medulla oblongata, just below the margin of the pons Variolii.

Deep Origin.-By two roots from the floor of the fourth ventricle, each root consisting of a number of gray filaments, some of which decussate in the median line; the external root has a gangliform enlargement containing fusiform nerve-cells.

Distribution.-The two roots wind around the restiform bodies and enter the internal auditory meatus, and divide into an anterior branch, distributed to the cochlea, and a posterior branch, distributed to the vestibule and semicircular canals.

Properties.-They are soft in consistence, grayish in color, consisting of axis-cylinders with a medullary sheath only; they are not sensible to ordinary impressions, but convey the impression of sound. 
Function.-Governs the sense of hearing. Receives and conducts to the brain the impression of sound, which gives rise to the sensations of hearing.

\section{Ninth Pair. Glossopharyngeal.}

Apparent Origin.-Partly from the medulla oblongata and the inferior peduncles of the cerebellum.

Deep Origin.-From the lower portion of the gray substance in the floor of the fourth venticle.

This nerve has two ganglia: The jugular ganglion includes only a portion of the root-filaments; the ganglion of Andersch includes all the fibers of the trunk.

Distribution.-The trunk of the nerve passes downward and forward, receiving near the ganglion of Andersch fibers from the facial and pneumogastric nerves. It divides into two large branches, one of which is distributed to the base of the tongue, the other to the pharynx. In its course it sends filaments to the otic ganglion; a tympanic branch which gives sensibility to the mucous membrane of the fenestra rotunda, fenestra ovalis, and Eustachian tube; lingual branches to the base of the tongue; palatal branches to the soft palate, uvula, and tonsils; pharyngeal branches to the mucous membrane of the pharynx.

Properties.-Irritation of the roots at their origin calls forth evidences of pain; it is, therefore, a sensory nerve, but its sensibility is not so acute as that of the trifacial. Irritation of the trunk after its exist from the cranium produces contraction of the muscles of the palate and pharynx, owing to the presence of anastomosing motor fibers.

Division of the nerve abolishes sensibility in the structures to which it is distributed and impairs the sense of taste in the posterior third of the tongue (see Sense of Taste).

Function.-Governs sensibility of pharynx, presides partly over the sense of taste, and controls reflex movements of deglutition and vomiting.

\section{Tenth Pair. Pneumogastric. Par Vagum.}

Apparent Origin.-From the lateral side of the medulla oblongata, just behind the olivary body.

Deep Origin.-In the gray nuclei in the lower half of the floor of the fourth ventricle and in the substance of the restiform body. Some filaments are traced along the restiform tract, toward the cerebellum, and 
others to the median line of the floor of the fourth ventricle, where many of them decussate.

This nerve has two ganglia: one in the jugular foramen, called the ganglion of the root, and another outside of the cranial cavity on the trunk, the ganglion of the trunk.

Distribution.-The filaments from the roots unite to form a single trunk, which leaves the cavity of the cranium, through the jugular foramen, in company with the spinal accessory and glossopharyngeal. It soon receives an anastomotic branch from the spinal accessory, and afterward branches from the facial, the hypoglossal, and the anterior branches of the two upper cervical nerves.

As the nerve passes down the neck it sends off the following main branches :

I. Pharyngeal nerves, which assist in forming the pharyngeal plexus, which is distributed to the nucous membrane and to the muscles of the pharynx.

2. Superior laryngeal nerve, which enters the larynx through the thyrohyoid membrane, and is distributed to the mucous membrane lining the interior of the larynx, and to the cricothyroid muscle and the inferior constrictor of the pharynx. The "depressor nerve," found in the rabbit, is formed by the union of two branches, one from the superior laryngeal, the other from the main trunk; it passes downward to be distributed to the heart.

3. Inferior laryngeal, which sends its ultimate branches to all the intrinsic muscles of the larynx except the cricothyroid, and to the inferior constrictor of the pharynx.

4. Cardiac branches given off from the nerve throughout its course, which unite with the sympathetic fibers to form the cardiac plexus, to be distributed to the heart.

5. Pulmonary branches, which form a plexus of nerves, and are distributed to the bronchi and their ultimate terminations, the lobules and air-cells. From the right pneumogastric nerve branches are distributed to the mucous membrane and muscular coats of the stomach and intestines, and to the liver, spleen, kidneys, and suprarenal capsules.

Properties.-At its origin the pneumogastric nerve is sensory, as shown by direct irritation or galvanization, though its sensibility is not very marked. In its course it exhibits motor properties, from anastomosis with motor nerves.

The pharyngeal branches assist in giving sensibility to the mucous 
membrane of the pharynx, and influence reflex phenomena of deglutition through motor fibers which they contain, derived from the spinal accessory.

The superior laryngeal nerve endows the upper portion of the larynx with sensibility; protects it from the entrance of foreign bodies; by conducting impressions to the medulla, excites the reflex movements of deglutition and respiration; through the motor filaments it contains, produces contraction of the cricothyroid muscle.

Division of the "depressor nerve" and galvanization of the central end retard and even arrest the pulsations of the heart, and by depressing the vaso-motor center, diminish the pressure of blood in the large vessels, by causing dilatation of the intestinal vessels through the splanchnic nerves.

The inferior laryngeal contains, for the most part, motor fibers from the spinal accessory. When irritated, produces movement in the laryngeal muscles. When divided, is followed by paralysis of these muscles, except the cricothyroid, impairment of phonation, and an embarrassment of the respiratory movements of the larynx, and, finally, death from suffocation.

The cardiac branches, through filaments derived from the spinal accessory, exert a direct inhibitory action upon the heart. Division of the pneumogastrics in the neck increases the frequency of the heart's action. Galvanization of the peripheral ends diminishes the heart's pulsations, and, if sufficiently powerful, paralyzes it in diastole.

The pulmonary branches give sensibility to the bronchial mucous membrane and govern the movements of respiration. Division of both pneumogastrics in the neck diminishes the frequency of the respiratory movements, which may fall as low as four to six a minute; death usually occurs in from five to eight days. Feeble galvanization of the central ends of the divided nerves accelerates respiration; " powerful galvanization retards, and may even arrest, the respiratory movements.

The gastric branches give sensibility to the mucous coat, and through sympathetic filaments, which join the pneumogastrics high up in the neck, give motion to the muscular coat of the stomach. They influence the secretion of gastric juice, aid the process of digestion and absorption from the stomach.

The hepatic branches, probably through anastomosing sympathetic filaments, influence the secretion of bile and the glycogenic function of the liver; division of the pneumogastrics in the neck produces congestion of the liver, diminishes the density of the bile, and arrests the glycogenic function; galvanization of the central ends exaggerates the glycogenic function and makes the animal diabetic. 
The intestinal branches give sensibility and motion to the small intestines, and when divided, purgative generally fail to produce evacuation.

Function. $-\Lambda$ great sensitive nerve, which, through anastomotic filaments from motor sources, influences deglutition, the action of the heart, the circulatory and respiratory systems, voice, the secretions of the stomach, intestines, and various glandular organs.

\section{Eleventh Pair. Spinal Accessory.}

Apparent Origin.-By two sets of filaments :

I. A bulbar or medullary set, four or five in number, from the lateral or motor tract of the lower half of the medulla oblongata, below the origin of the pneumogastric.

2. A spinal set, from six to eight in number, from the lateral portion of the spinal cord, between the anterior and posterior roots of the upper four or five cervical nerves.

Deep Origin.-The medullary portion arises in a nucleus in the lower half of the floor of the fourth ventricle, common to the pneumogastric and glossopharyngeal nerves. The spinal portion has its origin in an elongated nucleus lying along the external surface of the anterior cornua of the spinal cord, extending down to the fifth cervical vertebra.

Distributior..-From this origin the fibers unite to form a main trunk, which enters the cranial cavity through the foramen magnum, where it is at times joined by fibers from the posterior roots of the two upper cervical nerves, and sends filaments to the ganglion of the root of the pneumogastric. After emerging from the cranial cavity through the jugular foramen, it sends a branch to the pneumogastric and receives others in return, and also from the second, third, and fourth cervical nerves. It divides into two branches :

I. An internal or anastomotic branch, made up of filaments coming principally from the medulla oblongata; it is distributed to the muscles of the pharynx through the pharyngeal nerves coming from the pneumogastric ; to all the muscles of the larynx, except the cricothyroid, through the inferior laryngeal nerve; to the heart, by filaments which reach it through the pneumogastric nerve.

2. An external branch, which is distributed to the sterno-cleido-mastoid and trapezius muscles; these muscles also receive filaments from the cervical nerves. 
Properties.-At its origin it is a purely motor nerve, but in its course it exhibits some sensibility, which it received from anastomosing fibers.

Destruction of the medullary root, by tearing it from its attachment by means of forceps, impairs the action of the muscles of deglutition and destroys the power of producing vocal sounds by paralysis of the laryngeal muscles, without, however, interfering with the respiratory movements of the larynx, these being controlled by other motor nerves. The normal rate of movement of the heart is also impaired by destruction of the medullary root.

Irritation of the external branch throws the trapezius and sternomastoid muscles into convulsive movements, though section of the nerve does not produce complete paralysis, as they are also supplied with motor influence from the cervical nerves. The sternomastoid and trapezius muscles perform movements antagonistic to those of respiration, fixing the head, neck, and upper part of the thorax, and delaying the expiratory movement during the acts of pushing, pulling, straining, etc., and in the production of a prolonged vocal sound, as in singing. When the external branch alone is divided, in animals, they experience shortness in breath during exercise, from a want of coördination of the muscles of the limbs and respiration; and while they can make a vocal sound, it can not be prolonged.

Function.-Governs phonation by its influence upon the vocal movements of the glottis ; influences the movements of deglutition, inhibits the action of the heart, and controls certain respiratory movements associated with sustained or prolonged muscular efforts and phonation.

\section{Twelfth Pair. Hypoglossal or Sublingual.}

Apparent Origin.-By two groups of filaments from the medulla oblongata, in the grooves between the olivary body and the anterior pyramid.

Deep Origin.-From the hypoglossal nucleus, situated deep in the substance of the medulla, on a level with the lowest portion of the floor of the fourth ventricle; some decussating filaments have been traced to a higher encephalic center.

Distribution.-The trunk formed by a union of the root filament passes out of the cranial cavity through the anterior condyloid foramen, occasionally receiving a filament from the lateral and posterior portion of the medulla oblongata. After emerging from the cranium, it sends filaments to the sympathetic and pneumogastric; it anastomoses with the lingual branch of the fifth pair, and receives and sends filaments to the upper cer- 
vical nerves. The nerve is finally distributed to the sternohyoid, sternothyroid, omohyoid, thyrohyoid, styloglossi, hyoglossi, geniohyoid, geniohyoglossi, and the intrinsic muscles of the tongue.

Properties.-A purely motor nerve at its origin, but derives sensibility outside the cranial cavity from anastomosis with the cervical, pneumogastric, and fifth nerves.

Irritation of the nerve gives rise to convulsive movements of the tongue and slight evidences of sensibility.

Division of the nerve abolishes all movements of the tongue and interferes considerably with the act of deglutition.

When the hypoglossal nerve is involved in hemiplegia, the tip of the tongue is directed to the paralyzed side when the tongue is protruded, owing to the unopposed action of the geniohyoglossus on the sound side.

Articulation is considerably impaired in paralysis of this nerve, great difficulty being experienced in the pronunciation of the consonantal sounds.

Mastication is performed with difficulty, from inability to retain the food between the teeth until it is completely triturated.

Function.-Governs all the movements of the tongue and influences the functions of mastication, deglutition, and articulaton.

\section{MEDULLA OBLONGATA.}

The medulla oblongata is the expanded portion of the upper part of the spinal cord. It is pyramidal in form and measures $I \frac{1}{2}$ inches in length, $3 / 4$ of an inch in breadth, $1 / 2$ of an inch in thickness, and is divided into two lateral halves by the anterior and posterior median fissures, which are continuous with those of the cord. Each half is again subdivided by minor grooves into four columns-viz:, anterior pyramid, lateral and tract olivary body, restiform body, and posterior pyramid.

1. The anterior pyramid is composed partly of fibers continuous with those of the anterior column of the spinal cord, but mainly of fibers derived from the lateral tract of the opposite side by decussation. The united fibers then pass upward through the pons Varolii and crura cerebri, and for the most part terminate in the corpus striatum and cerebrum.

2. The lateral tract is continuous with the lateral columns of the cord; its fibers in passing upward take three directions-viz., an external bundle joins the restiform body, and passes into the cerebellum; an internal 
bundle decussates at the median line and joins the opposite anterior pyramid; a middle bundle ascends beneath the olivary body, behind the pons, to the cerebrum, as the fasciculus teres. The olivary body of each side is an oval mass, situated between the anterior pyramid and restiform body; it is composed of white matter externally and gray matter internally, forming the corpus dentatum.

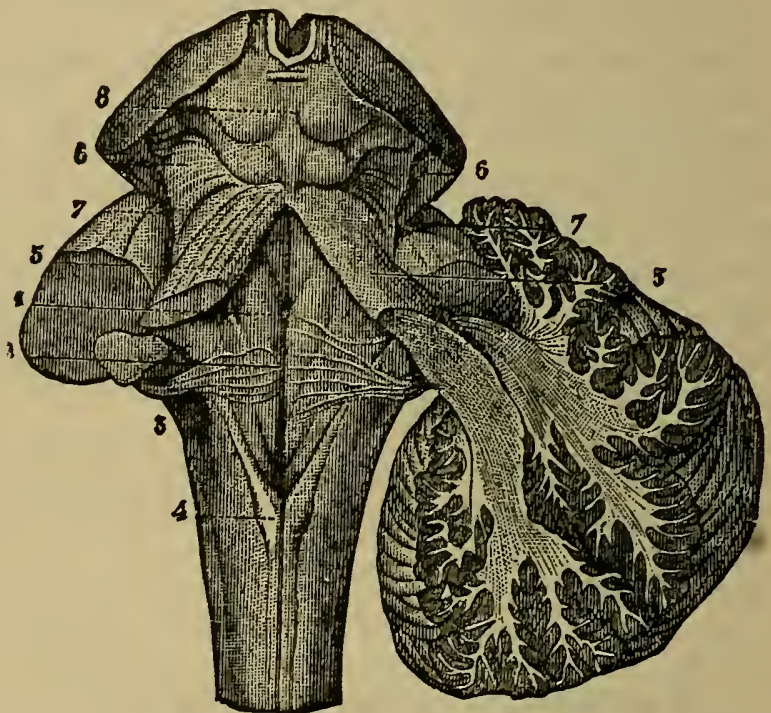

Fig. 23.-View of Cerebellum in Section, and of Fourth Ventricle, with the Neighboring Parts.-(From Sappey.)

1. Median groove fourth ventricle, ending below in the calamus scriptorizes, with the longitudinal eminences formed by the fasciculi teretes, one on each side. 2. The same groove, at the place where the white streaks of the auditory nerve emerge from it to cross the floor of the ventricle. 3. Inferior peduncle of the cerebellum, formed by the restiform body. 4. Posterior pyramid; above this is the calamus scriptorius. 5 , 5. Superior peduncle of cerebellum, or processus $e$ cerebello ad testes. 6.6 . Fillet to the side of the crura cerebri. 7, 7. Lateral grooves of the crura cerebri. 8. Corpora quadrigemina. (After Hirschfeld and Leveille.)

3. The restiform body, continuous with the posterior column of the cord, also receives fibers from the lateral column. As the restiform bodies pass upward they diverge and form a space (the fourth ventricle), the floor of which is formed by gray matter, and then turn backward and enter the cerebellum.

4. The posterior pyramid is a narrow white cord bordering the posterior median fissure; it is continued upward, in connection with the fasciculus teres, to the cerebrum. 
The gray matter of the medulla is continuous with that of the cord. It is arranged with much less regularity, becoming blended with the white matter of the different columns, with the exception of the anterior. By the separation of the posterior columns the transverse commissure is exposed, forming part of the floor of the fourth ventricle; special collections of gray matter are found in the posterior portions of the medulla, connected with the roots of origin of different cranial nerves.

Properties and Functions.-The medulla is excitable anteriorly and sensitive posteriorly to direct irritation. It serves-

1. As a conductor of sensitive impressions upward from the cord, through the gray matter to the cerebrum.

2. As a conductor of voluntary impulses from the brain to the spinal cord and nerves, through its anterior pyramids.

3. As a conductor of coördinating impulses from the cerebellum, through the restiform bodies to the spinal cord.

As an Independent Reflex Center.-The medulla oblongata contains special collections of gray matter, constituting independent nerve centers presiding over different functions, some of which are as follows-viz.:

I. A center which controls the movements of mastication, through afferent and efferent nerves. (See̊ p. 96.)

2. A center reflecting impressions which influence the secretion of saliva. (See p. 99.)

3. A center for sucking, mastication, and deglutition, whence are derived motor stimuli exciting to action and coördinating the muscles of the palate, pharynx, and esophagus, necessary for the swallowing of the food.

The secretion of sativa is also controlled by a center in the medulla.

NERVOUS CIRCLE OF DEGLUTITION.

Second and Third Stages.

$\begin{gathered}\text { Excitor } \\ \text { or } \\ \text { centripetal } \\ \text { nerves. }\end{gathered}$
Motor
$\begin{gathered}\text { or } \\ \text { centrifugal } \\ \text { nerves. }\end{gathered}$$\quad\left\{\begin{array}{l}\begin{array}{l}\text { Palatal branch of the fifth pair. } \\ \text { Pharyngeal branches of the glossopharyngeal. } \\ \text { Superior laryngeal branches of the pneumogastric. } \\ \text { Esophageal branches of the pneumogastric. }\end{array} \\ \begin{array}{l}\text { Pharyngeal branches of the pneumogastric, derived from } \\ \text { the spinal accessory. }\end{array} \\ \begin{array}{l}\text { Inferior or recurrent laryngeal. } \\ \text { Motor filaments of the third division of the fifth pair. } \\ \text { Portio dura. }\end{array}\end{array}\right.$


4. A center which coördinates the muscles concerned in the act of vomiting.

5. A speech center, coördinating the various muscles necessary for the accomplishment of articulation through the hypoglossal, facial nerves, and the second division of the fifth pair.

6. A center for the harmonization of muscles concerned in expression, reflecting its impulses through the facial nerve.

7. A cardiac center, which exerts (I) an accelerating influence over the heart's pulsations through accelerating nerve-fibers emerging from the cervical portion of the cord, entering the inferior cervical ganglion, and thence passing to the heart; (2) an inhibitory or retarding influence upon the action of the heart, through fibers of the spinal accessory nerve running in the trunk of the pneumogastric. The cardio-inhibitory center is in a state of tonic excitement and continuously sends impulses to the heart which exert an inhibitory influence upon its action. It may be stimulated directly by anemia as well as by venous hyperemia of the blood-vessels of the medulla and increased venosity of the blood. It is excited reflexly by the stimulation of the central end of the vagus, sciatic, and splanchnic nerves.

8. A vaso-motor center, which, by alternately contracting and dilating the blood-vessels through nerves distributed in their walls, regulates the quantity of blood distributed to an organ or tissue, and thus influences nutrition, secretion, and calorification. The vaso-motor center is situated in the medulla oblongata and pons Varolii, between the corpora quadrigemina and the calamus scriptorius. The vaso-motor fibers having their origin in this center descend through the interior of the cord, emerge through the anterior roots of spinal nerves, enter the ganglia of the sympathetic, and thence pass to the walls of the blood-vessels, and maintain an arterial tonus; they may be divided into two classes-viz., vasodilators (e.g., chorda tympani) and vaso-constrictors (e.g., sympathetic fibers).

Division of the cord at the lower border of the medulla is followed by a dilatation of the entire vascular system and a marked fall of the blood pressure. Galvanic stimulation of the divided surface of the cord is followed by a contraction of the blood-vessels and a rise in the blood pressure.

The vaso-motor center is stimulated directly by the condition of the blood in the medulla oblongata. When the blood is highly venous this center becomes very active, the blood-vessels throughout the body are contracted, and the blood current becomes swifter; sudden anemia of the medulla has a similar effect. The action of the vaso-motor center may be 
accelerated, with attendent rise of blood pressure, by irritation of certain afferent nerve-fibers. These are known as pressor fibers. On the other hand, its action may be depressed by other fibers, with attendant fall of blood pressure. These are known as depressor fibers.

9. A diabetic center, irritation of which causes an increase in the amount of urine secreted and the appearance of a considerable quantity of sugar in the urine.

10. Respiratory center, situated near the origin of the pneumogastric nerves, presides over the movements of respiration and its modifications, laughing, singing, sobbing, sneezing, etc. It may be excited reflexly by the presence of carbonic acid in the lungs irritating the terminal pneumogastric filaments; or automatically, according to the character of the blood circulating through it; an excess of carbonic acid or a diminution of oxygen increasing the number of respiratory movements; a reverse condition diminishing the respiratory movements.

11. A spasm center, stimulation of which gives rise to convulsive phenomena, such as coughing, sneezing, etc.

12. A center for certain ocular functions, governing the closure of the eyelids and dilatation of the pupil.

13. A sweat center is also localized in the medulla.

NERVOUS CIRCLE OF RESPIRATION.

Entirely Reflex.

Excitor
or
centripetal
nerves. $\quad\left\{\begin{array}{l}\text { Pulmonary branches of the pneumogastric. } \\ \text { Superior laryngeal. } \\ \text { Trifacial, or fifth pair. } \\ \text { Nerves of general sensibility. } \\ \text { Sympathetic nerve. }\end{array}\right.$

Motor
or
$\begin{gathered}\text { centrifugal } \\ \text { nerves. }\end{gathered}$$\left\{\begin{array}{l}\text { Phrenic, distributed to the diaphragm. } \\ \text { Intercostals, distributed to the intercostal muscles. } \\ \text { Facial nerve, or portio dura, to the facial muscles. } \\ \text { External branch of spinal accessory, to the trapezius and } \\ \text { sterno-cleido-mastoid muscles. }\end{array}\right.$

\section{PONS VAROLII.}

The pons Varolii unites the cerebrum above, the cerebellum behind, and the medulla oblongata below. It consists of transverse and longitudinal fibers, amidst which are, irregularly scattered collections of gray or vesicular nervous matter. 
The transverse fibers unite the two lateral halves of the cerebellum. The longitudinal fibers are continuous-

I. With the anterior pyramids of the medulla oblongata, which, interlacing with the deep layers of the transverse fibers, ascend to the crura cerebri, forming their superficial or fasciculated portions.

2. With fibers derived from the olivary fasciculus, some of which pass to the tubercula quadrigemina, while others, uniting with fibers from the lateral and posterior columns of the medulla, ascend in the deep or posterior portions of the crura cerebri.

Properties and Functions.-The superficial portion is insensible and inexcitable to direct irritation; the deeper portion appears to be excitable, consisting of descending motor fibers; the posterior portions are sensible, but inexcitable to irritation.

Transmits motor impulses and sensory impressions from and to the cerebrum.

The gray ganglionic matter consists of centers which convert impressions into conscious sensations and originate motor impulses, these taking place independent of any intellectual process; they are the seat of instinctive reflex acts, the centers which assist in the coördination of the automatic movements of station and progression.

\section{CRURA CEREBRI.}

The crura cerebri are largely composed of the longitudinal fibers of the pons (anterior pyramids, fasciculi teretes); after emerging from the pons they increase in size, and become separated into two portions by a layer of dark-gray matter, the locus niger.

The superfuial portion, the crusta, composed of the anterior pyramids, constitutes the motor tract, which terminates, for the most part, in the corpus striatum, but to some extent, also, in the cerebrum; the deep portion, made up of the fasciculi teretes and posterior pyramids and accessory fibers from the cerebellum, constitutes the sensory tract (the tegmentum), which terminates in the optic thalamus and cerebrum.

Function.-The crura are conductors of motor impulses and sensory impressions; the gray matter, the locus niger, assists in the coördination of 
the complicated movements of the eyeball and iris, through the motor oculi communis nerve. They also assist in the harmonization of general muscular movements, section of one crus giving rise to peculiar movements of rotation and somersaults forward and backward.

\section{CORPORA QUADRIGEMINA.}

The corpora quadrigemina are four small, rounded eminences, two on each side of the median line, situated immediately behind the third ventricle, and beneath the posterior border of the corpus callosum.

The anterior tubercles are oblong from before backward, and larger than the posterior, which are hemispheric in shape; they are grayish in color, but consist of white matter externally and gray matter internally.

Both the anterior and posterior tubercles are connected with the optic thalami by commissural bands, named the anterior and posterior brachia, respectively. They receive fibers from the olivary fasciculus and fibers from the cerebellum, which press upward to enter the optic thalami.

The corpora geniculata are situated, one on the inner side and one on the outer side of each optic tract, behind and beneath the optic thalamus, and from their position are named the corpora geniculatu interna and externa; they give origin to fibers of the optic nerve.

Functions.-The corpora quadrigemina are centers associated with the visual centers. Destruction of these tubercles is immediately followed by a loss of the sense of sight; moreover, their action in vision is crossed, owing to the decussation of the optic tracts, so that if the tubercle of the right side be destroyed by disease or extirpated, in a pigeon, the sight is lost in the eye of the opposite side, and the iris loses its mobility.

The tubercula quadrigemina as nerve centers preside over the reflex movements which cause a dilatation or contraction of the iris, irritation of the tubercles causing contraction, destruction causing dilatation. Removal of the tubercles on one side produces a temporary loss of power of the opposite side of the body, and a tendency to move around an axis is manifested, as after a section of one crus cerebri, which, however, may be due to giddiness and loss of sight.

They also assist in the coördination of the complex movements of the eye, and regulate the changes of the iris during the movements of accommodation for distance. 


\section{CORPORA STRIATA AND OPTIC THALAMI.}

The corpora striata are two large ovoid collections of gray matter, situated at the base of the cerebrum, the larger portions of which are embedded in the white matter, the smaller portions projecting into the anterior part of the lateral ventricle. Each striated body is divided, by a narrow band of white matter, into two portions-viz. :

I. The caudate mucleus, the intraventricular portion, which is conic in shape, having its apex directed backward, as a narrow, tail-like process.

2. The lenticular nucleus, embedded in the white matter, and for the most part external to the ventricle. On the outer side of the lenticular nucleus is found a narrow band of white matter, the external capsule; and between it and the convolutions of the island of Reil, a thin band of gray matter, the claustrum.

The corpora striata are grayish in color, and when divided, present transverse striations, from the intermingling of white fibers and gray cells.

The optic thalami are two oblong masses situated in the ventricles posterior to the corpora striata, and resting upon the posterior portion of the crura cerebri. The internal surface, projecting into the lateral ventricles, is white, but the interior is grayish, from a commingling of both white fibers and gray cells. Separating the lenticular nucleus from the caudate nucleus and the optic thalamus is a band of white tissue, the internal capsule.

The internal capsule is a narrow, curved tract of white matter, and is, for the most part, an expansion of the motor tract of the crura cerebri. It consists of two segments-an anterior, situated between the caudate nucleus and the anterior surface of the lenticular nucleus, and a posterior, situated between the optic thalamus and the posterior surface of the lenticular nucleus. These two segments unite at an obtuse angle, which is directed toward the median line. Pathologic observation has shown that the nervefibers of the direct and crossed pyramidal tracts can be traced upward through the anterior two thirds of the posterior segment into the centrum ovale, where, for the most part, they are lost ; a portion, however, remaining united, ascend higher and terminate in the paracentral lobule, the superior extremity of the ascending frontal and parietal convolutions. Those of the sensory tract can be traced upward, through the posterior third, into the cerebrum, where they probably terminate in the hippocampus major and uncinate convolution.

Functions. - The corpora striata are the centers in which terminate some of the fibers of the superficial or motor tract of the crura cerebri; 
others pass upward through the intervial capsule, to be distributed to the cerebrum. It might be inferred, from their anatomic relations, that the corpora striata are motor centers. Irritation by a weak galvanic current produces muscular movements of the opposite side of the body ; destruction of their substance by a hemorrhage, as in apoplexy, is followed by a paralysis of motion of the opposite side of the body, but there is no loss of sensation. When the hemorrhagic destruction involves the fibers of the anterior two thirds of the posterior segment of the internal capsule, and thus separates them from their trophic centers in the cortical motor region, a descending degeneration is established, which involves the direct pyramidal tract of the same side and the crossed pyramidal tract of the opposite side.

Destruction of the posterior one third of the posterior segment of the internal capsule is followed by a loss of sensation on the opposite side of the body and a loss of the senses of smell and vision on the same side (Charcot). The precise function of the corpora striata is unknown, but they are in some way connected with motion.

The optic thalami receives the fibers of the tegmentum, the posterior portion of the crura cerebri. They are insensible and inexcitable to direct irritation. Removal of one optic thalamus, or destruction of its substance by disease or hemorrhage, is followed by a loss of sensibility of the opposite side of the body, but there is no loss of motion; their precise function is also unknown, but they are in some way connected with sensation. In both cases their action is crossed.

\section{CEREBELLUM.}

The cerebellum is situated in the inferior fossæ of the occipital bone, beneath the posterior lobes of the cerebrum. It attains its maximum weight, which is about five ounces, between the twenty-fifth and fortieth years, the proportion between the cerebellum and cerebrum being as I to $8 \frac{4}{7}$.

It is composed of two lateral hemispheres and a central elongated lobe, the vermiform process; the two hemispheres are connected with each other by the fibers of the middle peduncle, forming the superficial portion of the pons Varolii. The cerebellum is brought into connection with the medulla oblongata and spinal corl through the prolongation of the restiform bodies; with the cerebrum, by fibers passing upward beneath the corpora quadrigemina and the optic thalami, and then forming part of the diverging cerebral fibers. 
Structure.-It is composed of both white and gray matter, the former being internal, the latter external, and is convoluted, for economy of space.

The zuhite matter consists of a central stem, the interior of which is a dentated capsule of gray matter, the corpus dentatum. From the external surface of the stem of white matter processes are given off, forming the lamine, which are covered with gray matter.

The gray matter is convoluted and covers externally the laminated processes; a vertical section through the gray matter reveals the following st uctures :

I. A delicate connective-tissue layer, just beneath the pia mater, containing rounded corpuscles, and with branching fibers passing toward the external surface.

2. The cells of Purkinje, forming a layer of large, nucleated, branched nerve-cells sending off processes to the external layer.

3. A granular layer of small but numerous corpuscles.

4. A nerve-fiber layer, formed by a portion of the white matter.

Properties and Functions.-Irritation of the cerebellum is not followed by any evidences either of pain or convulsive movements; it is, therefore, insensible and inexcitable.

Coördination of Movements. - Removal of the superficial portions of the cerebellum in pigeons produces feebleness and zuant of harmony in the muscular movements; as successive slices are removed, the movements become more irregular, and the pigeon becomes restless; when the last portions are removed, all power of Aying, walking, standing, etc., is entirely gone, and the equilibrium can not be maintained, the power of coördinating muscular movements being wholly lost. The same results have been obtained by operating on all classes of animals.

The following symptoms were noticed by Wagner, after removing the whole or a large part of the cerebellum :

1. A tendency on the part of the animal to throw itself on one side, and to extend the legs as far as possible.

2. Torsion of the head on the neck.

3. Trembling of the muscles of the body, which was general.

4. Vomiting and occasional liquid evacuations.

Forced Movements.-Division of one crus cerebelli causes the animal to fall on one side and roll rapidly on its longitudinal axis. According to Schiff, if the peduncle be divided from behind, the animal falls on the same side as the injury; if the section be made in front, the animal turns to the opposite side. 
Disease of the cerebellum partially corroborates the result of experiments ; in many cases symptoms of unsteadiness of gait, from $a$ want of coördination, have been noticed.

Comparative anatomy reveals a remarkable correspondence between the development of the cerebellum and the increase in complexity of muscular actions. It attains a much greater development, relatively to the rest of the brain, in those animals whose movements are very complex and varied in character, such as the kangaroo, shark, and swallow.

The cerebellum may possibly exert some influence over the sexual functions, but physiologic and pathologic facts are opposed to the idea of its being the seat of the sexual instinct. It appears to be simply a center for the coördination and equilibration of muscular movements.

\section{CEREBRUM.}

The cerebrum is the largest portion of the encephalic mass, constituting about four fifths of its weight; the average weight of the adult male brain is from forty-eight to fifty ounces, or about three pounds, while that of the adult female is about five ounces less. After the age of forty the weight of the cerebrum gradually diminishes at the rate of one ounce every ten years. In idiots the brain weight is often below the normal, at times not amounting to more than twenty ounces.

The blood-supply to the cerebrum is unusually large, considering its comparative bulk, nearly one fifth of the entire volume of blood in the body being distributed to it by the carotid and vertebral arteries. These vessels anastomose so freely, and are so arranged within the cavity of the cranium, that an obstruction in one vessel will not interfere with the regular supply of blood to the parts to which its branches are distributed. A diminished amount, or complete cessation, of the supply of blood is at once followed by a suspension of its functional activity.

The cerebrum is connected with the pons Varolii and medulla oblongata through the crura cerebri, and with the cerebellum through the superior peduncles. It is divided into two lateral halves, or hemispheres, by the longitudinal fissure running from before backward in the median line; each hemisphere is composed of both white and gray matter, the former being internal, the latter external ; it covers the surfaces of the hemisphere which are infolded, forming convolutions, for economy of space. 


\section{Fissures.}

I. The fissure of Sylvius is one of the most important; it is the first to appear in the development of the fetal brain, being visible at about the third month ; in the adult it is quite deep and well marked, running from the under surface of the brain upward, outward, and backward, and forms a boundary between the frontal and temporosphenoid lobes.

2. The fissure of Rolando is second in importance, and runs from a point on the convexity near the median line transversely outward and downward toward the fissure of Sylvius, but does not enter it. It separates the frontal from the parietal lobe.

3. The parietal fissure, arising a short distance hehind the fissure of Rolando, upon the convexity of the hemisphere, runs downward and backward to its posterior extremity.

4. The parieto-occipital fissure separates the occipital from the parietal lobe. Beginning upon the outer surface of the cerebrum, it is continued on the mesial aspect downward and forward until it terminates in the calcarine fissure.

5. The callosomarginal fissure lies upon the mesial surface, where it runs parallel with the corpus callosum.

Secondary fissures of importance are found in different lobes of the cerebrum, separating the various convolutions. In the anterior lobe are found the precentral, superior frontal, and inferior frontal fissures; in the temporosphenoid lobes are found the first and second temporosphenoid fissure; in the occipital lobe, the calcarine and hippocampal fissures.

\section{Convolutions. Frontal Lobe.}

The ascending frontal convolution, situated in front of the fissure of Rolando, runs downward-and forward; it is continuous above with the anterior frontal, and below with the inferior frontal, convolution.

The superior frontal convolution is bounded internally by the longitudinal fissure, and externally by the superior frontal fissure; it is connected with the superior end of the frontal convolution, and runs downward and forward to the anterior extremity of the frontal lobe, where it turns backward, and rests upon the orbital plate of the frontal bone.

The middle frontal convolution, the largest of the three, runs from behind forward, along the sides of the lobe, to its anterior part; it is bounded above by the superior and below by the inferior frontal fissures.

The inferior frontal convolution winds around the ascending branch of the fissure of Sylvius, in the anterior and inferior portion of the cerebrum. 
Parietal Lobe.-The ascending parietal convolution is situated just behind the fissure of Rolando, running downward and forward; above, it

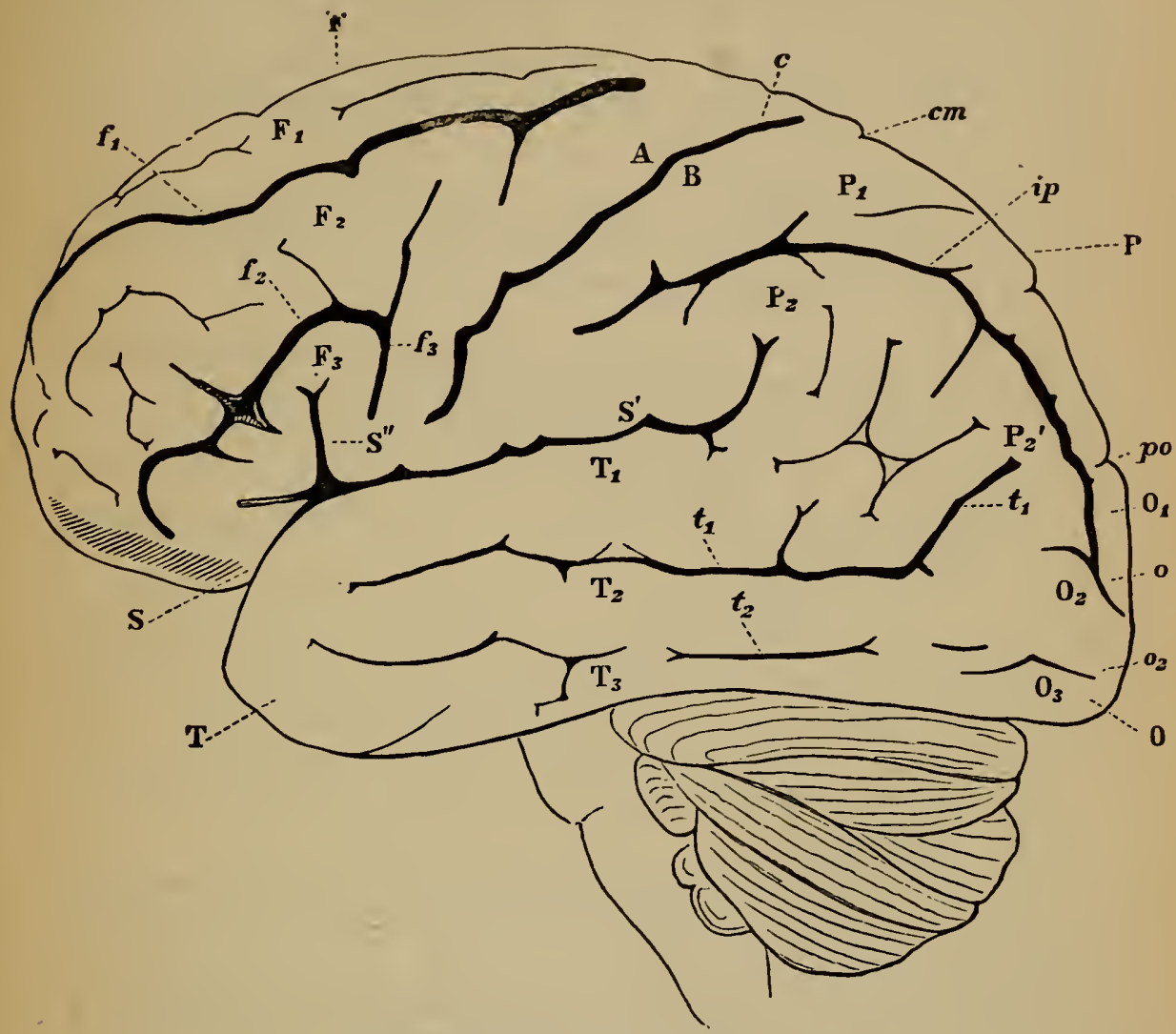

Fig. 24.-Diagram Showing Fissures and Convolutions of the Left Side of the Human Brain.-(Lanaois' "Physiorogy.")

F. Frontal. P. Parietal. O. Occipital. T. Temporosphenoid lobe. S. Fissure of Sylvius. S'. He rizontal. S'". Ascending ramus of S. c. Sulcus centralis, or fissure of Rolando. A. Ascending frontal, and B. Ascending parietal convolutions. $F_{1}$. Superior, $F_{2}$. Middle, and $F_{3}$. Inferior frontal convolutions. $f_{1}$. Superior, $f_{2}$. Inferior frontal fissures. $f_{3}$. Sulcus præcentralis. $P_{1}$. Superior paretial lobule. $\mathrm{P}_{2}$. Inferior parietal lobule, consisting of $\mathrm{P}_{2}$. Supramarginal gyrus, and $\mathrm{P}_{2}{ }^{\prime}$. Angular gyrus. ip. Sulcus interparit tali-. $\mathrm{cm}$. Terminat on of callosomarginal fissure. $\mathrm{O}_{1}$. First, $\mathrm{O}_{2}$. Second, $\mathrm{O}_{3}$. Third occipital convolutions. po. Parieto-occipiral fissure. 0 . Transverse occipital fissure. $o_{2}$. Inferior longitudinal occipital fissure. $\mathrm{T}_{1}$. First, $\mathrm{T}_{2}$. Second, $\mathrm{T}_{3}$. Temporospheno $\mathrm{d}$, convolutions. $t_{1}, t_{1}$. First, $t_{2}$. Second, temporosphenoid fissures.

becomes continuous with the upper parietal convolution, and below, winds around to be united with the ascending frontal. 
The upper parietal convolution is situated between the parietal and longitudinal fissures.

The supramarginal convolution winds around the superior extremity of the fissure of Sylvius.

The angular convolution, a continuation of the preceding, follows the parietal fissure to its posterior extremity, and then makes a sharp angle downward and forward.

Temporosphenoid Lobe.-Contains three well-marked convolutions, the superior, middle and inferior, separated by well-defined fissures, and continuous posteriorly with the convolutions of the parietal lobe.

The occipital lobe lies behind the parieto-occipital fissure, and contains the superior, middle and inferior convolutions, not well marked.

The central lobe, or island of Reil, situated at the bifurcation of the fissure of Sylvius, is a triangular-shaped cluster of six convolutions, the gyri operti, which are connected with those of the frontal, parietal, and temporosphenoid lobes.

Upon the inner or mesial aspect of the hemisphere are found (Fig. 25)I. The paracentral lobule, lying in the region of the upper extremity of the fissure of Rolando; it contains the large giant cells of Betz. Injury to this convolution is followed by degeneration of the motor tract.

2. The gyrus fornicatus, lying below the callosomarginal fissure. Running parallel with the corpus callosum, it terminates at its posterior border in the hippocampal gyrus.

3. The gyrus hippocampus $(\mathrm{H})$ is formed by the union of the preceding convolution with the occipitotemporal. It runs forward and terminates in a hooked extremity-uncus.

4. The quadrate lobule, or pracuneus, lies between the upper extremity of the callosomarginal fissure and the parieto-occipital.

5. The cuneus lies posteriorly to the quadrate lobule. It is a wedgeshaped mass inclosed by the calcarine and parieto-occipital fissures.

Structure.-The gray matter of the cerebrum, about $1 / 8$ of an inch thick, is composed of five layers of nerve-cells :

I. A superficial layer, containing a few small multipolar ganglion cells.

2. Small ganglion cells, pyramidal in shape.

3. A layer of large pyramidal ganglion cells with processes running off superiorly and laterally.

4. The granular formation containing nerve-cells.

5. Spindle-shaped and branching nerve-cells of a moderate size. 
The white matter consists of three distinct sets of fibers.

I. The diverging or peduncular fibers are mainly derived from the columns of the cord and medulla oblongata; passing upward through the crura cerebri, they receive accessory fibers from the olivary fasciculus, corpora quadrigemina, and cerebellum. Some of the fibers terminate in the optic

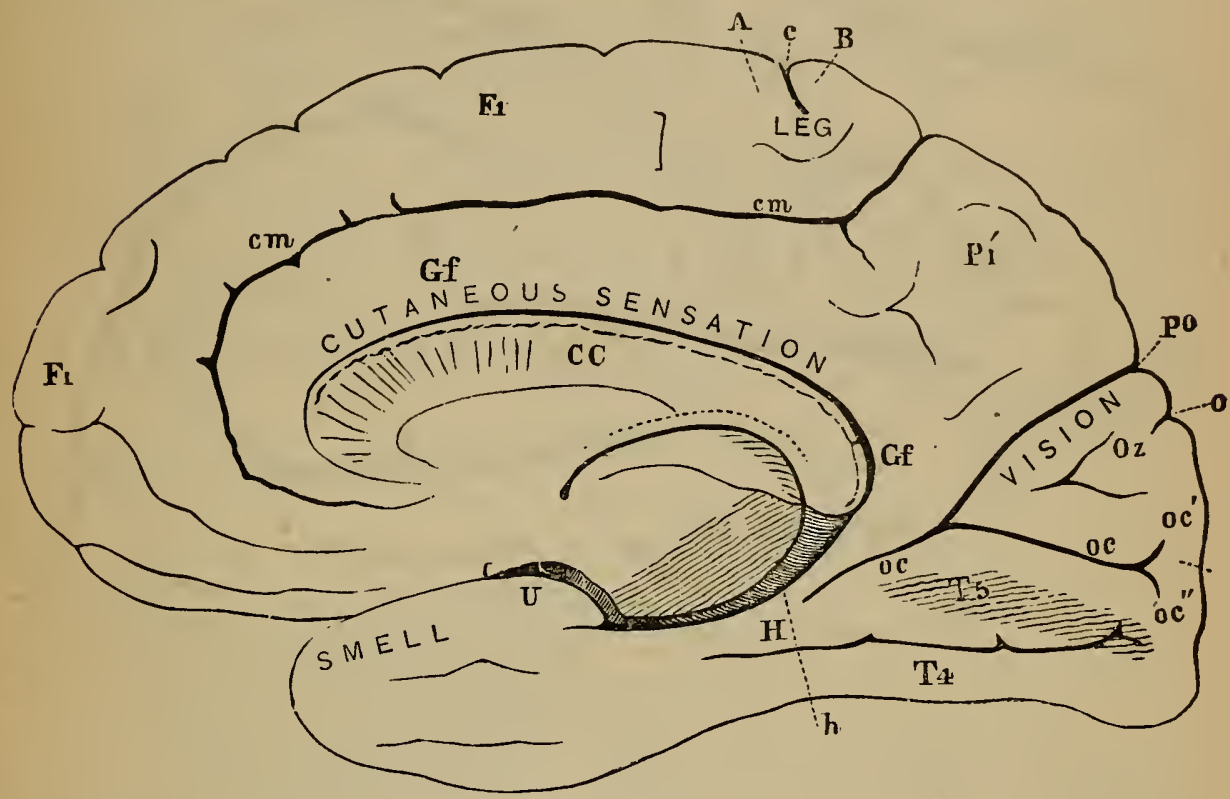

Fig. 25.-Diagram showing Fissures and Convolutions on Mesial Aspect of the Right Hemisphere.

Median aspect of the right hemisphere. CC. Corpus callosum divided longitudinallyi Gf. Gyrus fornicatus. H. Gyrus hippocampi. h. Sulcus hippocampi. U. Unc,nate gyrus. $\mathrm{cm}$. Callosomarginal fissure. $F_{1}$. First frontal convolution. $c$. Terminal portion of fissure of Rolando. A. Ascending frontal, B. Ascending parietal. convolution and paracential lobule. $\mathrm{P}_{1}^{\prime}$. Præcuneus or quadrate lobule. Oz. Cuneus. Po. Parieto-occipital fissure. o. Transverse occipital fissure. oc. Calcarine fissure. $o c^{\prime}$. Superior, $o c^{\prime \prime}$. Inferior, rami of the same. D. Gyrus descendens. $\mathbf{T}_{\mathbf{4}}$. Gyrus occipitotemporalis lateralis (lobulus fusiformis). $\mathbf{T}_{\mathbf{5}}$. Gyrus occipitotemporalis medialis (lobulus lingualis).

thalami and corpora striata, while others radiate into the anterior, middle, and posterior lobes of the cerebrum.

2. The transverse commissural fibers connect the two hemispheres, through the corpus callosum and anterior and posterior commissures.

3. The longitudinal commissural fibers connect different parts of the same hemisphere.

Functions.-The cerebral hemispheres are the centers of the nervous system through which are manifested all the phenomena of the mind; they 
are the centers in which impressions are registered and reproduced subsequently as ideas; they are the seat of intelligence, reason, and will.

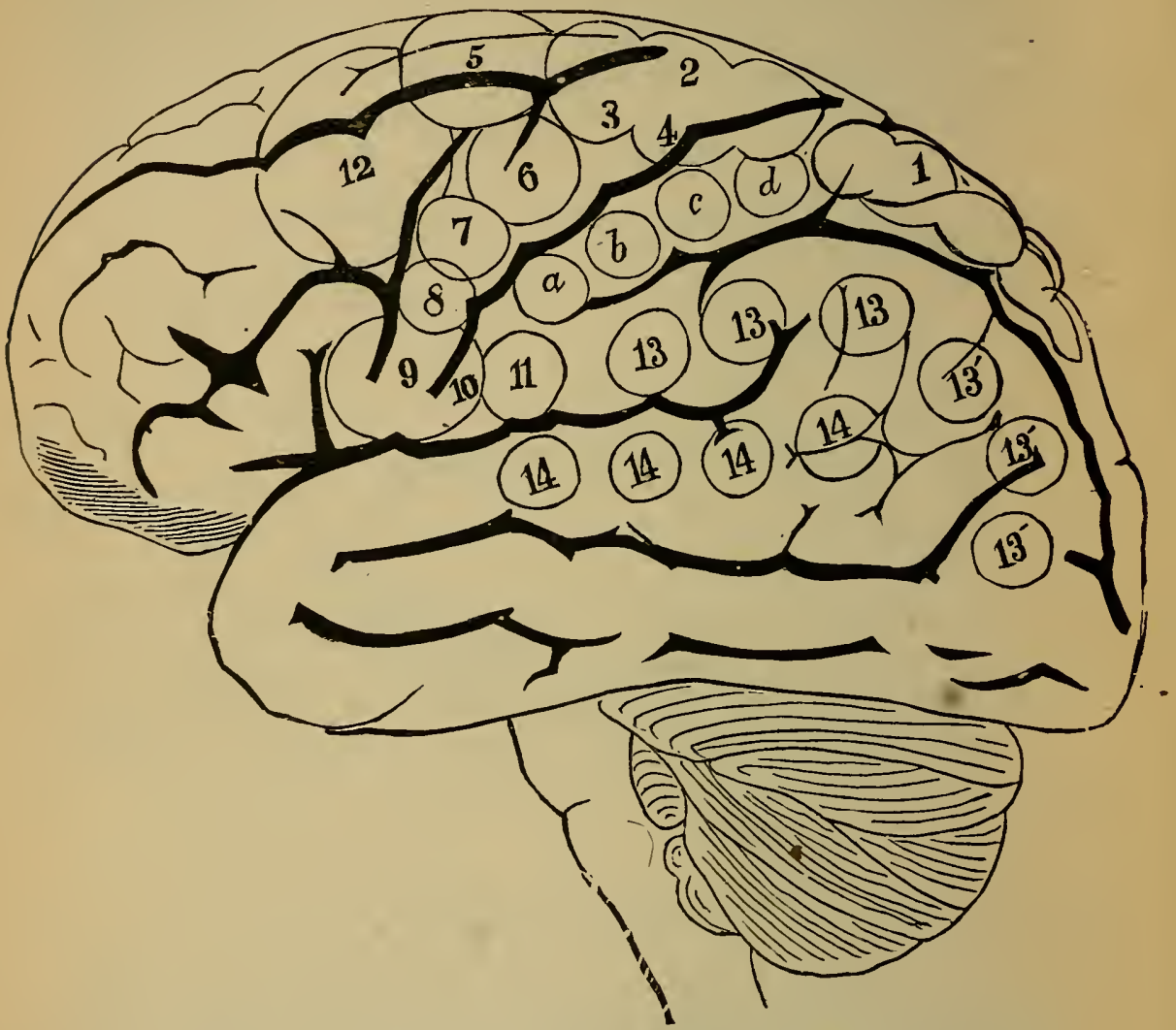

Fig. 26.-Side View of the Brain of Man, with the Areas of the Cerebral Convolutions aCCORDING TO FERRIER.

The figures are constructed by marking on the brain of man, in their respective situations, the areas of the brain of the monkey as determined by experiment, and the description of the effects of stimuiating the various areas refers to the brain of the monkey.

I. Advance of the opposite hind limb, as in walking. 2, 3, 4. Complex movements of the opposite leg and arm, and of the trunk, as in swimming. $a, b, c, d$. Individual and combined movements of the fingers and wrists of the opposite hand. Prehensile movements. 5. Extension forward of the opposite arm and hand. 6. Supination and flexion of the opposite forearm. 7. Retraction and elevation of the opposite angle of the mouth, by means of the zygomatic muscles. 8. Elevation of the alr nasi and upper lip, with depression of the lower lip on the opposite side. 9, 10. Opening of the mouth, with (9) protrusion and (10) retraction of the tongue : region of aphasia, bilateral action. TI. Retraction of the opposite angle of the mouth, the head turned slightly to one side. I2. The eyes open widely, the pupils dilate, and the head and eyes turn toward the opposite side. I3, I3'. The eyes moved toward the opposite side with an upward $\left({ }_{3}\right)$ or downward $\left({ }^{\prime} 3^{\prime}\right)$ deviation. The pupils are generally contracted. I4. Pricking of the opposite ear, the head and eyes turn to the opposite side, and the pupils diiate widely. 
However important a center the cerebrum may be for the exhibition of this highest form of nervous action, it is not directly essential for the continuance of life, for it does not exert any control over those automatic reflex acts, such as respiration, circulation, etc., which regulate the functions of organic life.

From the study of comparative anatomy, pathology, vivisection, etc., evidence has been obtained which throws some light upon the physiology of the cerebral hemispheres.

I. Comparative anatomy shows that there is a general connection between the size of the brain, its texture, the depth and number of convolutions, and the exhibition of mental power. Throughout the entire animal series, the increase in intelligence goes hand in hand with an increase in the development of the brain. In man there is an enormous increase in size over that of the highest animals, the anthropoids. The most cultivated races of men have the greatest cranial capacity ; that of the educated European being about II 6 cubic inches, that of the Australian being about 60 cubic inches, a difference of 56 cubic inches. Men distinguished for great mental power usually have large and well-developed brains; that of Cuvier weighed 64 ounces; that of Abercrombie, 63 ounces; the average being about 48 to 50 ounces. Not only the size, but, above all, the texture, of the brain must be taken into consideration.

2. Pathology.-Any severe injury or disease disorganizing the hemispheres is at once attended by a disturbance or an entire suspension of mental activity. A blow on the head, producing concussion, or undue pressure from cerebral hemorrhage, destroys consciousness; physical and chemic alterations in the gray matter have been shown to coexist with insanity, and with loss of memory, speech, etc. Congenital defects of organization from imperfect development are usually accompanied by a corresponding deficiency of intellectual power and of the higher instincts. Under these circumstances no great advance in mental development can be possible, and the intelligence remains of a low grade. In congenital idiocy not only is the brain of small size, but it is wanting in proper chemic composition, phosphorus, a characteristic ingredient of the nervous tissue, being largely diminished in amount.

3. Experimentation upon the lower animals by removing the cerebral hemispheres is attended by results similar to those observed in disease and injury. Removal of the cerebrum in pigeons produces complete abolition of intelligence, and destroys the capability of performing spontaneous movements. The pigeon remains in a condition of profound stupor, which is not accompanied, however, by a loss of sensation or of 
the power of producing reflex or instinctive movements. The pigeon can be temporarily aroused by pinching the feet, loud noises, lights placed before the eyes, etc., but soon relapses into a state of quietude, being unable to remember impressions and connect them with any train of ideas, the faculties of memory, reason, and judgment being completely abolished.

\section{CEREBRAL LOCALIZATION OF FUNCTION.}

From experiments made upon animals, and from the results of clinical and post-mortem observations upon men, it has been shown that the phenomena of organic and psychic life are presided over by anatomically localized centers in the brain. A knowledge of the position of these centers becomes of the highest importance in localizing the seat of lesions, thrombi, hemorrhages, new growths, etc., which show themselves in paralyses, epilepsies, etc. It has not been possible to thus localize all functions, and to many parts of the brain no special use can be assigned. The following are the centers most definitely mapped out and that are of paramount importance.

Motor Centers.- These are in the cortical gray matter, and are arranged along either side of the fissure of Rolando. This area is known as the motor area or motor zone, stimulation of which is followed by convulsive movements of the muscles of the opposite side of the body, while destruction of the gray matter of this area is followed by permanent paralysis of the muscles of the opposite side. From experiments made upon monkeys, Ferrier has mapped out a number of motor centers which he has transferred to corresponding localities on the human brain. (See Fig. 26.) The descriptive text of the illustration renders his results intelligible. Pathologic studies have largely confirmed his deductions. In a general way it may be said that the upper third of the ascending frontal and parietal convolutions about this fissure preside over the movements of the leg of the opposite side of the body; the middle third controls the movements of the arm; the upper part of the inferior third is the facial area. The lowest part of the inferior third governs the motility of the lips and tongue, and this space, with the posterior extremity of the third frontal convolution, constitutes the speech center.

The experiments of Horsley and Schäfer have enabled them to furnish a new diagrammatic representation of the motor area and more accurately 
to define the special areas upon the lateral and mesial aspects of the brain of the monkey. The boundaries of the general and special areas, as determined by these observers, will be readily understood by an examination of Figures 27 and 28 .

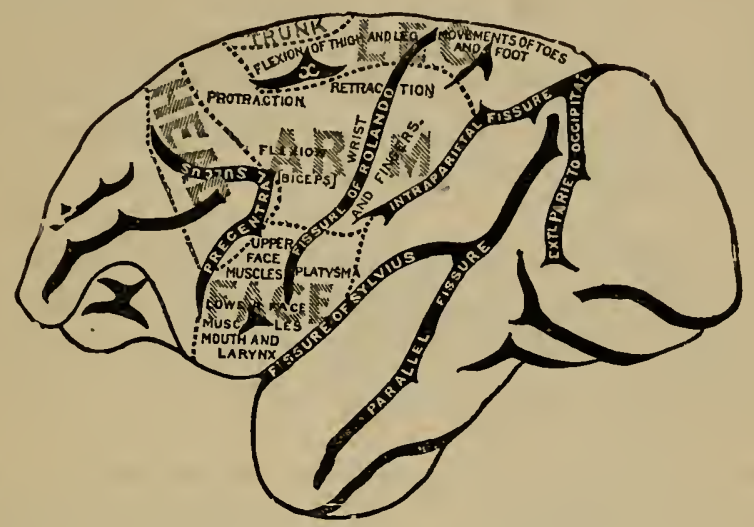

Fig. 27.-Diagram of the Motor Areas on the Outer Surface of a Monkey's BRAIN-( Horsley and Schäfer.)

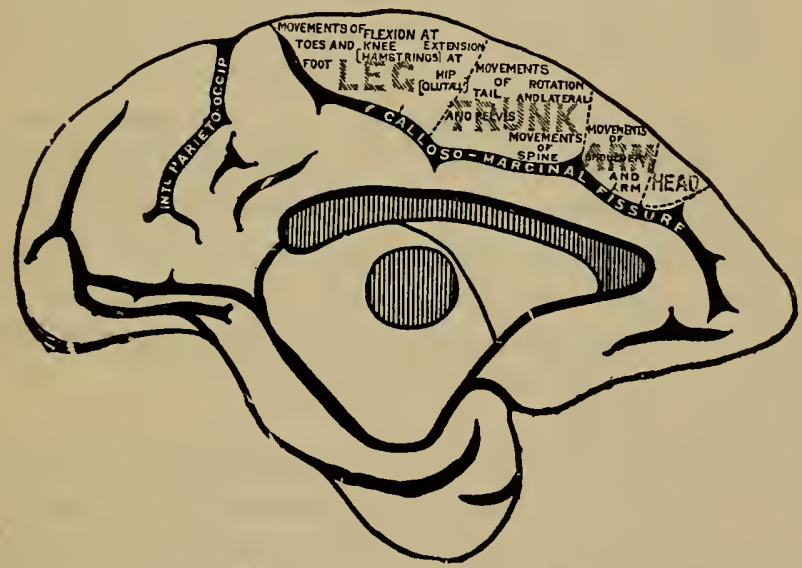

Fig. 28.-Diagram of the Motor Arras on the Marginal Convolution of a MONKRY'S BRAIN.-(Horsley and Schäfer.)

For diagnostic purposes the motor areas for the face and limbs have been subdivided as follows :

I. The face area may be divided into an upper part, comprising about one third, and a lower part, comprising the remaining two thirds. In the upper part are centers governing the movements of the muscles of the 
ofposite angle of the mouth and of the lower face. The anterior portion of the lower two thirds controls the movements of the vocal cords, and may be regarded as a laryngeal center; the posterior portion governs the opening and shutting of the mouth and the protrusion and retraction of the tongue.

2. The upper limb area may be subdivided as follows: The upper part controls the movements of the shoulder; posteriorly and below this point are centers for the elbow; below and anteriorly, centers for the wrist and finger movements, while lowest and posteriorly, centers governing the thumb.

3. The leg area may be subdivided as follows: The anterior part, both on the mesial and lateral surfaces, contains centers governing the hip and thigh movements; in the posterior part are centers for the movements of the leg and toes. The center for the great toe has been located in the paracéntral lobule.

4. The trunk area, situated largely on the mesial surface, contains anteriorly centers governing the rotation and arching of the spine, while -posteriorly are found centers governing movements of the tail and pelvis.

5. The head area, or area for visual direction, contains centers excitation of which causes "opening of the eyes, dilatation of the pupils, and turning of the head to the opposite side, with conjugate deviation of the eyes to that side."

The centers of origin of the nerves for the ocular muscles lie in the gray matter of the aqueduct of Sylvius. Destruction of the gray matter at these points is followed by paralysis of the muscles of the opposite side of the body, and morbid growths, hemorrhages, or thrombi of the vessels of the part result in abnormal stimulation or in interference with the functions corresponding to the nature and extent of the lesion. Cerebral or Jacksonian epilepsy is a result of local cortical disease.

Center for Speech.-Pathologic investigations have demonstrated that the left third frontal convolution is of essential importance for speech. Adjoining this convolution are the centers controlling the motility of the lips, tongue, etc. In the majority of cases the speech centers are on the left side of the brain, though in exceptional cases they are on the right side, especially in left-handed persons. In deaf mutes this convolution is very imperfectly developed, while in monkeys it is quite rudimentary.

Lesions of the third frontal convolution on the left side, if the patient be right-handed, produce the various forms of aphasia, or the partial or complete loss of the power of articulate speech. 
Aphasia is of many degrees and kinds. In ataxic aphasia the patient is unable to communicate his thoughts by words, there being an inability to execute the movements of the mouth, etc., necessary for speech. In agraphic aphasia there is an inability to execute the movements necessary for writing, though the mental processes are retained. In the ataxic form the lesion is in the third frontal convolution, and in the agraphic form it is in the arm center.

In amnesic aphasia there is a loss of the memory of words, the purest examples of which consist of the affections known as word-deafness and word-blindness. In word-deafness the patient can not understand vocal speech, though he is capable of hearing other sounds. This condition is associated with lesion of the first temporal convolution. In word-blindness the patient can not name a letter or a word when printed or written, though he can see all other objects. This condition is associated with impairment of the visual centers.

Figure 29 will illustrate the conditions in the various forms of aphasia. Impressions are constantly passing

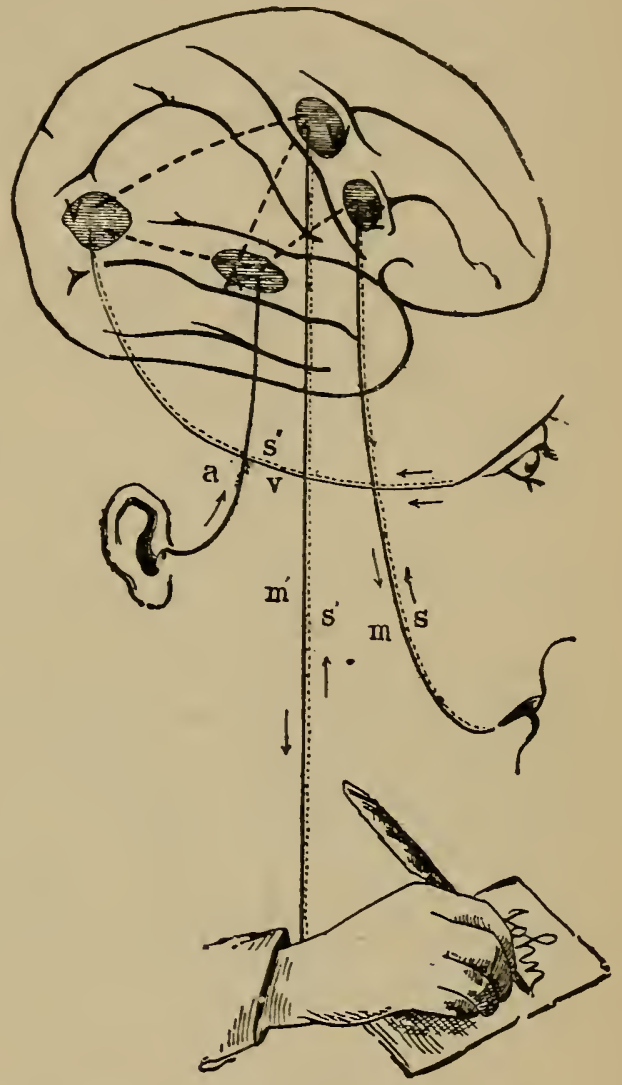

Frg. 29. from eye and ear to the visual and auditory centers and are there being registered. Commissural fibers connect these centers with the arm and speech centers, which in turn are connected by efferent fibers with the muscles of the hand and of the vocal apparatus. Muscular movements of the eyes, hand, and mouth are also registered by means of the afferent fibers, $s, s^{\prime}, s^{\prime \prime}$.

Sensory Centers.- These are the centers in which the sensory impres- 
sions are coördinated, and in which they probably become parts of our consciousness. The most important are :

The visual center, located in the occipital lobe and especially in the cuneus. Unilateral destruction of this area results in hemianopsia, or blindness of the corresponding halves of the two retinæ. Destruction of both occipital lobes in man results in total blindness. Stimulation or irritation of the visual center causes photopsia, or hallucinations of sight, in corresponding halves of the retinæ. There have been instances of injury of these parts when sensations of color were abolished with preservation of those of space and light, thus showing a special localization of the color center. Recent experiments show that the centers of the two hemispheres are united, as ocular fatigue of an unused eye was found to be proportional to the fatigue of the exercised one.

The auditory centers are located in the temporosphenoid lobes. Worddeafness is associated with softening of these parts, and their complete removal results in deafness.

The gustatory and olfactory centers are located in the uncinate gyrus, on the inner side of the temporosphenoid lobes. There does not seem to be any differentiation, up to this time, of these two centers.

The center for tactile impressions was located by Ferrier in the hippocampal region. Horsley and Schäfer found that destructive lesions of the gyrus fornicatus were followed by hemianesthesia of the opposite side of the body, which was more or less marked and persistent. These observers conclude that the limbic lobe "is largely, if not exclusively, concerned in the appreciation of sensations, painful and tactile."

The superior and middle frontal convolutions appear to be the seats of the reason, intelligence, and will. Destruction of these parts is followed by proportional hebetude, without any impairment of sensation or motion.

\section{SYMPATHETIC NERVOUS SYSTEM.}

The sympathetic nervous system consists of a chain of ganglia connected by longiturdinal nerve filaments, situated on each side of the spinal column, running from above downward. The two ganglionic cords are connected in the interior of the cranium by the ganglion of Ribes, on the anterior communicating artery, and terminate in the ganglion impar, situated at the top of the coccyx.

The chain of ganglia is divided into groups, and named according to the 
location in which they are found-viz., cranial, four in number; cervical, three; thoracic, twelve; lumbar, five; sacral, five; coccygeal, one. Each ganglion consisis of a collection of vesicular nervous matter, bundles of non-medullated nerve-fibers, embedded in a capsule of connective tissue. The ganglia are reinforced by motor and sensory fibers from the cerebrospinal nervous system.

The ganglia have distinct nerve-fibers, from which branches are distributed to the glands, arteries, and muscles, and to the cerebral and spinal nerves ; many pass, also, to the visceral ganglia-e.g., cardiac, semilunar, pelvic, etc.

\section{Cephalic Ganglia.}

I. The ophthalinic or ciliary ganglion is situated in the orbital cavity, posterior to the eyeball; it is of small size and of a reddish-gray color; receives filaments of communication from the motor oculi, ophthalmic branch of the fifth pair, and the carotid plexus. Its filaments of distribution are the ciliary nerves, which consist of-

(a) Motor fibers for the circular fibers of the iris and ciliary muscle.

(b) Sensory fibers for the cornea, iris, and associated parts.

(c) Vaso-motor fibers for the blood-vessels of the choroid, iris, and retina.

(d) Motor fibers for the dilator fibers of the iris.

2. The spheno-palatine or Meckel's ganglion, triangular in shape, is situated in the sphenomaxillary fossa; receives filaments from the facial (Vidian nerve) and the superior maxillary branch of the fifth nerve. Its filaments of distribution pass to the gums, the soft palate, and the, levator palati and azygos uvulæ muscles.

3. The otic or Arnold's ganglion is of small size, oval in shape, and situated beneath the foramen ovale; receives a motor filament from the facial and sensory filaments from the glossopharyngeal and fifth nerves; sends filaments to the mucous membrane of the tympanic cavity and to the tensor tympani muscle.

4. The submaxillary ganglion, situated in the submaxillary gland, receives filaments from the chorda tympani, sensory filaments from the lingual branch of the fifth nerve, and filaments from the sympathetic. The chorda tympani nerve supplies vaso-dilator and secretory fibers to the submaxillary and sublingual glands. The fifth nerve endows the glands with sensibility, while the sympathetic supplies secretory or trophic fibers.

\section{Cervical Ganglia.}

The superior cervical ganglion is fusiform in shape, of a grayish-red 
color, and situated opposite the second and third cervical vertebræ; it sends branches to form the carotid and cavernous plexuses, which branches follow the course of the carotid arteries to their distribution; also sends branches to join the glossopharyngeal and pneumogastric, to form the pharyngeal plexus.

The middie cervical ganglion, the smallest of the three, is occasionally absent; it is situated opposite the fifth cervical vertebra; sends branches to the superior and inferior cervical ganglia and to the thyroid artery.

The inferior cervical ganglion, irregular in form, is situated opposite the last cervical vertebra; it is frequently united with the first thoracic ganglion.

The superior, middle, and inferior cardiac nerves, arising from these cervical ganglia, pass downward and forward to form the deep and superficial cardiac plexuses located at the bifurcation of the trachea, from which branches are distributed to the heart, coronary arteries, etc.

The thoracic ganglia are usually twelve in number, and are placed against the heads of the ribs behind the pleura; they are small in size and gray in color; they communicate with the cerebro-spinal nerves by two filaments, one of which is white, the other gray.

The great splanchnic nerve is formed by the union of branches from the sixth, seventh, eighth, and ninth ganglia; it passes through the diaphragm to the semilunar ganglion.

The lesser splanchnic nerve is formed by the union of filaments from the tenth and eleventh ganglia, and is distributed to the celiac plexus.

The renal splanchnic nerve arises from the last thoracic ganglion and terminates in the renal plexus.

The semilunar ganglia, the largest of the sympathetic system, are situated by the side of the celiac axis ; they send radiating branches to form the solar plexus; from the various plexuses, nerves follow the gastric, splenic, hepatic, renal, etc., arteries, into the different abdominal viscera.

The lumbar ganglia, four in number, are placed upon the bodies of the vertebra; they give off branches, which unite to form the aortic lumbar plexus and the hypogastric plexus, and follow the blood-vessels to their terminations.

The sacral and coccygeal ganglia send filaments of distribution to all the blood-vessels of the pelvic viscera.

Properties and Functions.-The sympathetic nerve possesses both sensibility and the power of exciting motion, but these properties are much less decided than in the cerebro-spinal system. Irritation of the ganglia 
does not produce any evidence of pain until some time has elapsed. If caustic soda be applied to the semilunar ganglia, or a galvanic current be passed through the splanchnic nerve, no instantaneous effect is noticed, as in the case of the cerebro-spinal nerves; but in the course of a few seconds a slow, progressive contraction of the muscular coat of the intestines is established, which continues for some time after the irritation has been removed. Division of the sympathetic nerve in the neck is followed by a vascular congestion of the parts above the section on the corresponding side, attended by an increase in the temperature; not only is there an increase in the amount of blood, but the rapidity of the blood current is very much accelerated and the blood in the veins becomes of a brighter color. Galvanization of the upper end of the divided nerve causes all the preceding phenomena to disappear; the congestion decreases, the temperature falls, and the venous blood becomes dark again.

The sympathetic exerts a similar influence upon the circulation of the limbs and the glandular organs; destruction of the first thoracic ganglion and division of the nerves forming the lumbar and sacral plexuses are followed by a dilatation of the vessels, an increased rapidity of the circulation, and an elevation of temperature in the anterior and posterior limbs; galvanization of the peripheral ends of these nerves causes all of these phenomena to disappear. Division of the splanchnic nerve causes a dilatation of the blood-vessels of the intestine.

These phenomena of the sympathetic nervous system are dependent upon the presence of vaso-motor nerves, which, under normal circumstances, exert a tonic influence upon the blood-vessels. These nerves, derived from the cerebro-spinal system and the medulla oblongata, leave the spinal cord by the rami communicantes, enter the sympathetic ganglia, and finally terminate in the muscular walls of the blood-vessels.

Sleep is a periodic condition of the nervous system in which there is a partial or complete cessation of the activities of the higher nerve centers. The cause of sleep is a diminution in the quantity of blood, occasioned by a contraction of the smaller arteries under the influence of the vaso-motor nerves.

During the waking state the brain undergoes a physiologic waste, as a result of the exercise of its functions; after a certain length of time its activities become enfeebled, and a period of repose ensues, during which a regeneration of its substance takes place.

When the brain becomes enfeebled, there is a diminished molecular activity and an accumulation of waste products ; under these circumstances 
it ceases to dominate the medulla oblongata and the spinal cord. These centers then act more vigorously, and diminish the caliber of the cerebral blood-vessels through the action of the vaso-motor nerves, producing a condition of physiologic anemia and sleep ; during this state waste products are removed, force is stored up, nutrition is restored, and waking finally occurs.

\section{THE SENSE OF TOUCH.}

The sense of touch is a modification of general sensibility, and is located in the skin, which is especially adapted for this purpose on account of the number of nerves and papillary elevations it possesses. The structures of the skin and the modes of termination of the sensory nerves have already been considered.

The tactile sensibility varies in acuteness in different ${ }^{-}$portions of the body, being most marked in those regions in which the tactile corpuscles are most abundant-e. $g$, , the palmar surface of the third phalanges of the fingers and thumb.

The relative sensibility of different portions of the body has been ascertained by means of a pair of compasses : the points of the instrument being guarded by cork, it was then determined how closely they could be brought together, and yet be felt at two different points. 'The following are some of the measurements :

Point of tongue, . . . . . . . 1 $1 / 2$ of a line.

Palmar surface of third phalanx, . . . . I line.

Red surface of lips, . . . . . . . . 2 lines.

Palmar surface of metacarpus, . . . . . 3 " "

Tip of the nose, . . . . . . . . 3 "

Part of lips covered by skin, . . . . . . 4 "

Palm of hand, .......... . . 5 "

Lower part of forehead, . . . . . . . IO "

Back of hand, ............ I4 "

Dorsum of foot, . . . . . . . 18 "

Middle of the thigh, ........ . . 30 "6

The sense of touch communicates to the mind the idea of resistance only, and the varying degrees of resistance offered to the sensory nerves enable us to estimate, with the aid of the muscular sense, the qualities of hardness or softness of external objects. The idea of space or extension is obtained when the sensory surface or the external object changes its place in regard 
to the other; the character of the surface, its rougrhness or smoothness, is estimated by the impressions made upon the tactile papillæ.

Appreciation of Temperature.-The general surface of the body is more or less sensitive to differences of temperature, though this sensation is separate from that of touch; whether there are nerves especially adapted for the conduction of this sensation has not been fully determined. Under pathologic conditions, however, the sense of touch may be abolished, while the appreciation of changes in temperature may remain normal.

The cutaneous surface varies in its sensibility to temperature in different parts of the body, and depends, to some extent, upon the thickness of the skin, exposure, habit, etc. ; the inner surface of the elbow is more sensitive to changes in temperature than the outer portion of the arm ; the left hand is more sensitive than the right, the mucous membrane less so than the skin.

Excessive heat or cold has the same effect upon the sensibility; the temperatures most readily appreciated are those between $50^{\circ} \mathrm{F}$. and $I I 5^{\circ} \mathrm{F}$.

The sensations of pain and tickling appear to be conducted to the brain, also, by nerves different from those of touch; in abnormal conditions the appreciation of pain may be entirely lost while touch remains unimpaired.

\section{THE SENSE OF TASTE.}

The sense of taste is localized mainly in the mucous membrane covering the superior surface of the tongue.

The tongue is situated in the floor of the mouth; its base is directed backward and is connected with the hyoid bone and by numerous muscles with the epiglottis and soft palate; its apex is directed forward against the posterior surface of the teeth.

The substance of the tongue is made up of intrinsic muscle-fibers, the linguales; it is attached to surrounding parts, and its various movements are performed by the extrinsic muscles- $e_{.} g$., styloglossus, geniohyoglossus, etc.

The mucous membrane covering the tongue is continuous with that lining the commencement of the alimentary canal, and is furnished with vascular and nervous papillæ.

The papilla are analogous in their structure to those of the skin, and are distributed over the dorsum of the tongue, giving it its characteristic roughness. 
There are three principal varieties-

I. The filiform papilla are most numerous, and cover the anterior two thirds of the tongue; they are conic or filiform in shape, often prolonged into filamentous tufts, of a whitish color, and covered by horny epithelium.

2. The fungiform papille are found chiefly at the tip and sides of the tongue; they are larger than the preceding, and may be recognized by their deep red color.

3. The circumvallate papille are rounded eminences, from eight to ten in number, situated at the base of the tongue, where they form a $V$-shaped figure. They are quite large, and consist of a central projection of mucous membrane, surrounded by a wall, or circumvallation, from which they derive their name.

The taste-beakers, supposed to be the true organs of taste, are flasklike-bodies, ovoid in form, about $\frac{1}{30} \overline{0}$ of an inch in length, situated in the epithelial covering of the mucous membrane, on the circumvallate papillæ. They consist of a number of fusiform, narrow cells, which are curved so as to form the walls of this flask-like body; in the interior are elongated cells, with large, clear nuclei, the taste-cells.

Nerves of Taste.-The chorda tympani nerve, a branch of the facial, after leaving the cavity of the tympanum, joins the third division of the fifth nerve between the two pterygoid muscles, and then passes forward in the lingual branches, to be distributed to the mucous membrane of the anterior two thirds of the tongue. Division or disease of this nerve is followed by a loss of taste in the part to which it is distributed.

The glossopharyngeal enters the tongue at the posterior border of the hyoglossus muscle, and is distributed to the mucous membrane of the base and sides of the tongue, fauces, etc.

The lingual branch of the trifacial nerve endows the tongue with general sensibility ; the hypoglossal endows it with motion.

The nerves of taste in the superficial layer of the mucous membrane form a fine plexus, from which branches pass to the epithelium and penetrate it; others enter the taste-beakers, and are directly connected with the taste-cells.

The seat of the sense of taste has been shown by experiment to be the whole of the mucous membrane over the dorsum of the tongue, soft palate, fauces, and upper part of the pharynx.

The sense of taste enables us to distinguish the savor of substances 
introduced into the mouth, which faculty is different from tactile sensibility, The sapid qualities of substances appreciated by the tongue are designated as bitter, sweet, alkaline, sour, salt, etc.

The essential conditions for the production of the impressions of taste are :

I. A state of solubility of the food.

2. A free secretion of the saliva, and

3. Active morements on the part of the tongue, exciting pressure against the roof of the mouth, gums, etc., thus aiding the solution of various articles and their osmosis into the lingual papillæ.

Sapid substances, when in a state of solution, pass into the interior of the taste-beakers, and come into contact, through the medium of the tastecells, with the terminal filaments of the gustatory nerves.

\section{THE SENSE OF SMELL.}

The sense of smell is located in the mucous membrane lining the upper part of the nasal cavity, in which the olfactory nerves are distributed.

The nasal fossæ are two cavities, irregular in shape, separated by the vomer, the perpendicular plate of the ethmoid bone, and the triangular cartilage. They open anteriorly and posteriorly by the anterior and posterior nares, the latter communicating with the pharynx. They are lined by mucous membrane, of which the only portion capable of receiving odorous impressions is the part lining the upper one third of the fossæ.

The olfactory nerves, arising by three roots from the posterior and inferior surface of the anterior lobes, pass forward to the cribriform plate of the ethmoid bone, where they each expand into an oblong body, the olfactory bulb. From its under surface from fifteen to twenty filaments pass downward through the foramina, to be distributed to the olfactory mucous membrane, where they terminate in long, delicate, spindle-shaped cells, the olfactory cells, situated between the ordinary epithelial cells.

The olfactory bulbs are the centers in which odorous impressions are perceived as sensations, destruction of these bulbs being attended by an abolition of the sense of smell.

In animals which possess an acute sense of smell there is a corresponding increase in the development of the olfactory bulbs. 
The essential conditions for the sense of smell are--

1. A special nerve center capable of receiving impressions and transforming them into odorous sensations.

2. Emanations from bodies which are in a gaseous or vaporous condition.

3. The odorous emanations must be drawn freely through the nasal fossæ; if the odor be very faint, a peculiar inspiratory movement is made, by which the air is forcibly brought into contact with the olfactory filaments. The secretions of the nasal fossæ probably dissolve the odorous particles. Various substances, as ammonia, horseradish, etc., excite the sensibility of the mucous membrane; this must be distinguished from the perception of true odors.

\section{THE SENSE OF SIGHT.}

The Eyeball.-The eyeball, or organ of vision, is situated at the fore part of the orbital cavity and is supported by a cushion of fat ; it is protected from injury by the bony walls of the cavity, the lids, and the lashes, and is so situated as to permit of an extensive range of vision. The eyeball is loosely held in position by a fibrous membrane, the capsule of Tenon, which is attached on the one hand to the eyeball itself and on the other to the walls of the cavity. Thus suspended, the eyeball is capable of being moved in any direction by the contraction of the muscles attached to jt.

Structure. - The eyeball is spheroid in shape and measures about $\frac{9}{10}$ of an inch in its anteroposterior diameter, and a little less in its transverse diameter. When viewed in profile, it is seen to consist of the segments of two spheres, of which the posterior is the larger, occupying five sixths, and the anterior the smaller, occupying one sixth, of the ball.

The eye is made up of several membranes, concentrically arranged, within which are inclosed the refracting media essential to vision. These membranes, enumerated from without inward, are-

I. The sclerotic and cornea.

2. The choroid and iris.

3. The retina.

The refracting media are the aqueous humor, the crystalline lens, and the vitreous humor.

The Sclerotic and Cornea.-The sclerotic is the opaque fibrous membrane covering the posterior five sixths of the ball. It is composed of connective tissue arranged in layers, which run both transversely and longitudinally; it is pierced posteriorly by the optic nerve about $\frac{1}{10}$ of an 
inch internal to the optic axis. The sclerotic, by its density, gives form to the eye and protects the delicate structures within it, and serves for the attachment of the muscles by which the ball is moved.

The cornea is a transparent non-vascular membrane covering the anterior one sixth of the eyeball. It is nearly circular in shape and is continuous at the circumference with the sclerotic, from which it can not be separated. The substance of the cornea is made up of thin layers of delicate, transparent fibrils of connective tissue, more or less united ; between these layers are found a number of intercommunicating lymph-spaces, lined by endothelium, which are in connection with lymphatics. Leukocytes or lynphcorpuscles are often found in these spaces. The anterior surface of the cornea is covered by several layers of nucleated epithelium, which rest upon a structureless membrane known as the anterior elastic lamina. The posterior surface is covered by a similar membrane, the membrane of Descemet, which at its periphery becomes continuous with the iris; it is also covered by a layer of epithelial cells. At the junction of the cornea and sclerotic is found a circular groove, the canal of Schlemm.

The choroid, the iris, the ciliary muscle, and the ciliary processes together constitute the second or middle coat of the eyeball.

The choroid is a dark brown membrane which extends forward nearly to the cornea, where it terminates in a series of folds, the ciliary processes. In its structure the choroid is highly vascular, consisting of both arteries and veins. Externally it is connected with the sclerotic by connective tissue; internally it is lined by a layer of hexagonal pigment cells, which, though usually classed as belonging to the choroid, is now known to belong, embryologically and physiologically, to the retina. From without inward may be distinguished the following layers :

I. The lamina suprachoroidea.

2. The elastic layer of Sattler, consisting of two endothelial layers.

3. The chorio-capillaris, choroid proper, or membrane of Ruysch-a thick, elastic network of arterioles and capillaries lying within the outer layer of veins and arteries called the venæ vorticosæ.

4. The lamina vitrea, or internal limiting membrane.

The choroid with its contained blood-vessels bears an important relation to the nutrition of the eye ; it provides for the blood-supply and for drainage from the body of the eye, and presents a uniform and high temperature to the retina.

The iris is the circular, variously colored membrane placed in the anterior portion of the eye just behind the cornea. It is perforated a little 
to the nasal side of the center by a circular opening, the pupil. The outer or circumferential border is connected with the cornea, ciliary muscle, and ciliary processes ; the free inner edge forms the boundary of the pupil, the size of which is constantly changing. The framework of the iris is composed of connective-tissue blood-vessels, muscle-fibers and pigmented connective-tissue corpuscles. The anterior surface is covered with a layer of ephithelial cells continuous with those covering the posterior surface of the cornea; the posterior surface is lined by a limiting membrane bearing pigment epithelial cells continuous with those of the choroid. The various

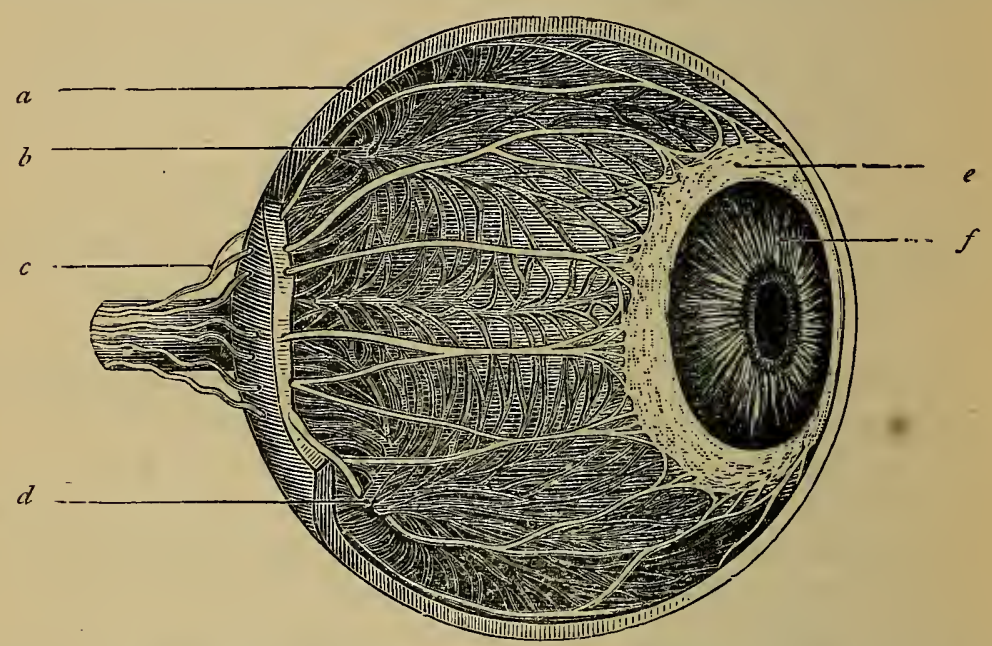

Fig. 30.-Sclerotic Coat removed to show Choroid Ciliary Muscle, and Nerves.-(From Holden's "Anatomy.")

$a$. Sclerotic coat. b. Veins of the choroid. $c$. Ciliary nerves. $d$. Veins of the choroid. e. Ciliary muscle. $f$. Iris.

colors which the iris assumes in different individuals depend upon the quantity and disposition of the pigment granules.

The muscle-fibers of the iris, which are of the non-striated variety, are arranged in two sets-the sphincter and dilator.

The sphincter pupille is a circular, flat band of muscle-fibers surrounding the pupil close to its posterior surface; by its contraction and relaxation the pupil is diminished or increased in size. The dilatator pupilla consists of a thin layer of fibers arranged in a radiate manner; at the margin of the pupil they blend with those of the sphincter muscle, while at the outer border they arch to form a circular muscular layer.

The ciliary muscle is a gray, circular band, consisting of unstriped 
muscle-fibers about $\frac{1}{10}$ of an inch long running from before backward. It is attached anteriorly to the inner surface of the sclerotic and cornea, and posteriorly to the choroid coat opposite the ciliary processes. At the anterior border of the radiating fibers and internally are found bundles of circular muscle-fibers, constituting the annular muscle of Muiller. The ciliary muscle thus consists of two sets of fibers, a radiating and a circular, both of which are concerned in effecting a change in the convexity of the lens in the accommodation of the eye to near vision.

The retina forms the internal coat of the eye. In the fresh state it is a delicate, transparent membrane of a pink color, but after death soon becomes opaque ; it extends forward almost to the ciliary processes, where it terminates in an indented border, the ora serrata. In the posterior part of the retina, at a point corresponding to the axis of vision, is a yellow spot, the macula lutea, which is somewhat oval in shape and tinged with yellow pigment. It presents in its center a depression, the fovea centralis, corresponding to a decrease in thickness of the retina; about $\frac{1}{10}$ of an inch to the inner side of the macula is the point of entrance of the optic nerves The arteria centralis retince pierces the optic nerve near the sclerotic, runs forward in its substance, and is distributed in the retina as far forward as the ciliary processes.

The retina is remarkably complex, consisting of ten distinct layers, from within outward, supported by connective tissue. These are as followsviz. :

I. Membrana limitans interna.

2. Fibers of optic nerve.

3. Layers of ganglionic corpuscles.

4. Molecular layer.

5. Internal granular layer.

6. Molecular layer.

7. External granular layer.

8. Membrana limitans externa.

9. Jacobson's membrane, or layer of rods and cones.

Io. The layer of pigment cells.

The most important of these, however, is the layer of rods and cones in the external portion of the retina. The rods are straight, elongated cylinders extending through the entire thickness of Jacobson's membrane. They consist of an external portion, which is clear, homogeneous, and bighly refracting, and of an internal portion, which is slightly granular and less refractive; the outer end of each rod is in direct contact with the pig- 
ment epithelium lining the choroid, while the inner end, tapering to a fine thread, pierces the external limiting membrane and passes into the external granular layer. 'The cones consist also of two portions, the inner of which is somewhat thicker than the rod and rests upon the limiting membrane; the outer portion tapers to a fine point, which is known as the cone-style. The cones, as a rule, are somewhat shorter than the rods. The proportion of rods to cones varies in different parts of the retina, though there are on an average about fourteen rods to one cone. In the macula lutea, where vision is most acute, the rods are almost entirely absent, cones alone being present. All the retinal elements at this point are changed. The nerve-fiber layer is absent, the axis-cylinders radiating in such a manner as to leave the spot free from their covering. The remaining layers are all thinned and the stroma is reduced to a minimum. The optic nerve, after passing forward from the brain, penetrates in succession the sclerotic, choroid, and retina; the nerve-fibers then spread out over the anterior surface of the retina and become connected with the large ganglionic cells, the third layer of the retina.

The number of optic nerve-fibers in the retina is estimated to be about 800,000 , and for each fiber there are about seven cones, one hundred rods, and seven pigment cells. The points of the rods and cones are directed toward the choroid, or away from the entering light, and dip into the pigment layer. They, with the pigment layer, are the intermediating elements in the change of the ethereal vibrations into nerve force; out of these nerve vibrations the brain fashions the sensations of light, form, and color.

The vitreous humor, which supports the retina, is the largest of the refracting media; it is globular in form and constitutes about four fifths of the ball; it is hollowed out anteriorly for the reception of the crystalline lens. The outer surface of the vitreous is covered by a delicate, transparent membrane, termed the hyaloid membrane, which serves to maintain its globular form.

The aqueous humor, found in the anterior chamber of the eye, is a clear alkaline fluid, having a specific gravity of 1003-1009. It is secreted most probably by the blood-vessels of the iris and ciliary processes. It passes from the interior of the eye, through the canal of Schlemm and the meshes at the base of the iris, into the anterior circular vein.

The crystalline lens, inclosed within its capsule, is a transparent biconvex body, situated just behind the iris and resting in the depression in the anterior part of the vitreous. The two convexities are not quite alike, the curvature of the posterior surface being slightly greater than that of the an- 
terior. The lens measures about $\frac{1}{3}$ of an inch in the transverse diameter and $\frac{1}{5}$ of an inch in the anteroposterior diameter.

The suspensory ligament, by which the lens is held in position, is a firm, transparent membrane, united to the ciliary processes. A short distance beyond its origin it splits into two layers, the anterior of which is inserted into the capsule of the lens and blends with it ; the posterior, passing inward behind the lens, becomes united to its capsule. The anterior layer presents a series of foldings, zone of Zinn, which are inserted into the intervals of the folds of the ciliary processes. The triangular space between the two layers is the canal of Petit.

Blood-vessels and Nerves.-The structures composing the eyeball are supplied with blood by the long and short ciliary arteries, branches of the ophthalmic; they pierce the sclerotic at various points and are ultimately distributed to all tissues within the ball.

The nerve-supply comes largely from the ophthalmic or ciliary ganglion. This is a small body, situated in the posterior part of the orbit; it receives motor fibers from a branch of the motor oculi, or third nerve; a sensory branch from the ophthalmic division of the fifth nerve, and fibers from the cavernous plexus of the sympathetic. From the anterior border of the ganglion proceed the ciliary nerves, which, entering the eyeball, endow its structures with motion and sensation.

The Eyeball a Living Camera Obscura.-The eyeball may be compared in a general way to a camera obscura. The anatomic arrangement of its structures reveals many points of similarity. The sclerotic and choroid may be compared with the walls of the chamber; the combined refractive media, cornea, aqueous $F$ mor, lens, and vitreous humor, to the lens for focusing the rays of light; the retina, to the sensitive plate receiving the image formed at the focal point; the iris, to the diaphragm, which, by cutting off the marginal rays, prevents spheric aberration and at the same time regulates the amount of light entering the eye; the ciliary muscle, to the adjusting screw, by which distinct images are thrown upon the retina in spite of varying distances of the object from which the light rays emanate. The structures just enumerated are those essential for normal vision.

The relationship of the various structures composing the eyeball is shown in Figure $\mathbf{3} \mathbf{1}$.

The dioptric or refracting apparatus, by which the rays of light entering the eye are so manipulated as to produce an image on the retina, consists of the cornea, aqueous humor, crystalline lens, and vitreous humor. A ray of light in passing through each of these media will undergo refraction 
at their surfaces and ultimately be brought to a focus at the retina. Inasmuch as the two surfaces of the cornea are parallel and its refractive power practically the same as the aqueous humor, the media may be reduced to three-viz.:

1. Cornea and aqueous humor.

2. The lens.

3. The vitreous humor.

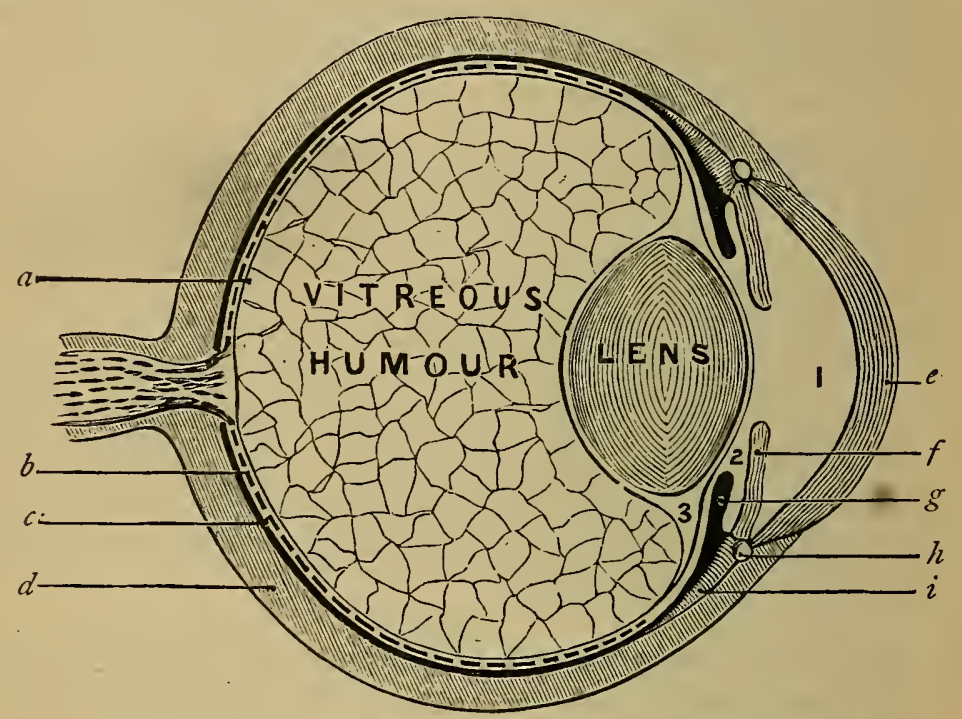

Fig. 3r.-Diagram of a Vertical Section of the Eye.-(From Holdens,

I. Anterior chamber filled with aqueous humor. 2. Posterior chamber. 3 Canal of

Petit. $a$. Hyaloid membrane. b. Retina (dotted line). c. Choroid coat (black

line). $d$. Sclerotic coat. e. Cornea. $f$. Iris. $g$. Ciliary processes. $h$. Canal of Schlemm or Fontana. $i$. Ciliary muscle.

The refracting surfaces may also be reduced to three-viz. :

I. Anterior surface of the cornea.

2. Anterior surface of lens.

3. Posterior surface of lens.

The refraction effected by the cornea is very great, owing to the passage of the light from the air into a comparatively dense mediun, and is sufficient of itself to bring parallel rays of light to a focus about ten millimeters behind the retina. This would be the condition in an eye in which the lens was congenitally absent. Perfect vision requires, however, that the convergence of the light shall be great enough to allow the image to fall upon the retina. 
This is accomplished by the crystalline lens, a body denser than the cornea and possessing a higher refractive power. The manner in which a biconvex lens focuses both parallel and divergent rays is shown in figures 32 and 33 .

The function of the crystalline lens, therefore, is to focus the rays of light with the formation of an image on the retina.

The retinal image corresponds in all respects to the object from which the light proceeds. The existence of this image can be demonstrated by removing from the eye of a recently killed animal a circular portion of the sclerotic and choroid posteriorly, and then placing at the proper distance in

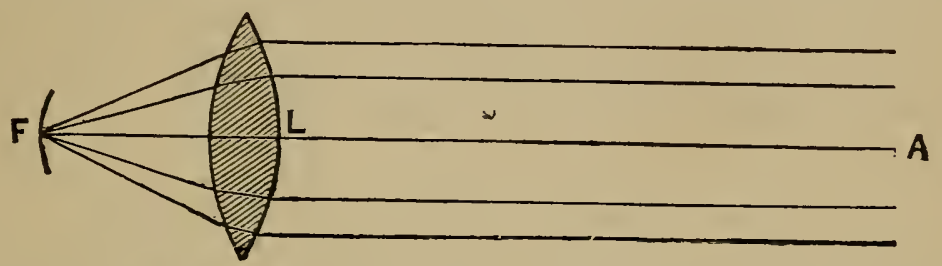

Fig. 32.-Diagrair showing the Course of Parallel Rays of Light from A, in their Passage through a Biconvex Lens, L, in which they are so Refrated as to Bind Toward and Come in a Focus at a Point, F. -(From Yeo's "Text-book of Physiology.")

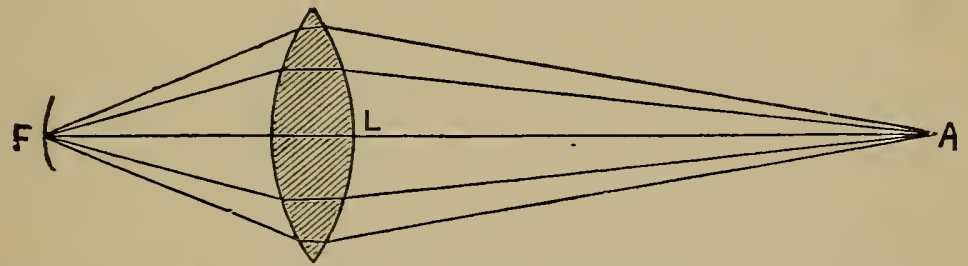

Fig. 33.-Diagram showing the Course of Diverging Rays which are Bent to a Point Further fron the Lens than the Parallel Rays in PreCEDING FIGURE.-(Yeo's "Text-book of Physiology.")

front of the cornea a lighted candle ; an inverted image of the candle will be seen upon the retina. The size of the retinal image depends upon the visual angle, which in turn depends upon the size of the object and its dis-

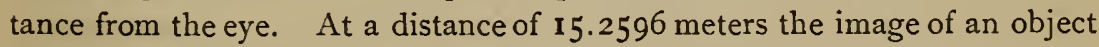
one meter high would be one millimeter, or a thousand times smaller than the object.

Accommodation.-By accommodation is understood the power which the eye possesses of adjusting itself to vision at different distances. In a normal or emmetropic eye parallel rays of light are brought to a focus on 
the retina ; but divergent rays - that is, rays coming from a near luminous point-will be brought to a focus behind the retina, provided the refractive media remain the same; as a result, vision would be indistinct, from the formation of diffusion circles. It is impossible to see distinctly, therefore, a near and a distant object at the same time. We must alternately direct the vision from one to the other. A normal eye does not require adjusting for parallel rays ; but for divergent rays a change in the eye is necessitated ; this is termed accommodation. In the accommodation for near vision the lens becomes more convex, particularly on its anterior surface. The increase in convexity augments its refractive power; the greater the degree of divergence of the rays previous to entering the eye, the greater the increase of convexity of the lens and convergence of the rays after passing through it. By this alteration in the shape of the lens we are enabled to focus light rays coming from, and to see distinctly, near as well as distant objects.

Function of the Ciliary Muscle.-Though it is admitted that the change in the convexity of the lens is caused by the contraction of the ciliary muscle and the relaxation of the suspensory ligament, the exact manner in which it does so is not understood. When the eye is in repose, as in distant vision, the suspensory ligament is tense, and the lens possesses that degree of curvature necessary for focusing parallel rays. In the voluntary efforts to accommodate the eye for near vision, the ciliary muscle contracts, the suspensory ligament relaxes, and the lens, inherently elastic, bulges forward and once again focuses the rays upon the retina. It is, therefore, termed the muscle of accommodation, and by its alternate contraction and relaxation the lens is rendered more or less convex, according to the requirements for near and distant vision.

Range of Accommodation.-Parallel rays coming from a luminous point distant not less than 200 feet do not require adjustment; from this point up to infinity no accommodation is required for perfect vision. This is termed the punctum remotum, and indicates the distance to which an object may be removed and yet distinctly seen. If the object be brought nearer to the eye than 200 feet, the accommodative power must come into play; the nearer the object, the more energetic must be the contraction of the ciliary muscle and the consequent increase in the convexity of the lens. At a distance of five inches, however, the power of accommodation reaches its maximum; this is termed the punctum proximum, and indicates the nearest point at which an object may be seen distinctly. The distance between these two points is the range of accommodation. 
Optic Defects. - Astigmatism is a cundition of the eye which prevents vertical and horizontal lines from being focused at the same time, and is due to a greater curvature of the cornea in one meridian than in another.

Spheric aberration is a condition in which there is an indistinctness of an image from the unequal refraction of the rays of light passing through the circumference and the center of the lens; it is corrected mainly by the iris, which cuts off the marginal rays, and transmits only those passing through the center.

Chromatic aberration is a condition in which the image is surrounded by a colored margin, from the decomposition of the rays of light into their elementary parts.

Myopia, or shortsightness, is caused by an abnormal increase in the anteroposterior diameter of the eyeball, or by a hypernormal refracting power of the lens. It is generally due to the first cause; the lens, being too far removed from the retina, forms the image in front of it, and the perception becomes dim and blurred. Concave glasses correct this defect by preventing the rays from converging too soon.

Hypermetropia, or longsightness, is caused by a shortening of the anteroposterior diameter or by a subnormal refractive power of the lens; the focus of the rays of light would, therefore, be behind the retina. Convex glasses correct this defect by converging the rays of light more anteriorly.

Presbyopic is a loss of the power of accommodation of the eye to near objects, and usually occurs between the ages of forty and sixty ; it is remedied by the use of convex glasses.

The Iris.-The iris plays the part of a diaphragm, and by means of its central aperture the pupil regulates the quantity of light entering the interior of the eye; by preventing rays from passing through the margin of the lens it diminishes spheric aberration. The size of the pupil depends upon the relative degree of contraction of the circular and radiating fibers; the variations in size of the pupil from variations in the degree of contraction depend upon different intensities of light. If the light be intense, the circular fibers contract, and diminish the size of the pupil; if the light diminishes in intensity, the circular fibers relax and the pupil enlarges.

Point of Most Distinct Vision.-While all portions of the retina are sensitive to light, their sensibility varies within wide limits. At the macula lutea, and more especially in its most central depression, the fovea, where the retinal elements are reduced practically to the layer of rods and 
cones, the sensibility reaches its maximum. It is at this point that the image is found when vision is most distinct. The macula and fovea are always in the line of direct vision. From the macula toward the periphery of the retina there is a gradual diminution in sensibility, and a corresponding decline in the distinctness of vision. In those portions of the retina lying outside the macula, the indistinctness of vision depends not only on diminished sensibility, but also upon inaccurate focusing of the rays.

Blind Spot.-Although the optic nerve transmits the impulses excited in the retina by the ethereal vibration, the nerve-fibers themselves are insensitive to light. At the point of entrance of the optic nerve, owing to the absence of the rods and cones, the rays of light make no impression. This is the blind spot. As this spot is not in the line of vision, no dark point is ordinarily observed in the field of vision-the circular space before a fixed eye within which reflections of objects are perceptible.

The rods and cones are the most sensitive portions of the retina. A ray of light entering the eye passes entirely through the various layers of the retina, and is arrested only upon reaching the pigmentary epithelium in which the rods and cones are embedded. As to the manner in which the objective stimuli-light and color, so called-are transformed into nerve impulses, but little is known. It is probable that the ethereal vibrations are transformed into heat, which excites the rods and cones. These, acting as highly specialized end organs of the optic nerve, start the impulses on their way to the brain, where the seeing process takes place. As to the relative function of the rods and cones, it has been suggested, from the study of the facts of comparative anatomy, that the rods are impressed only by differences in the intensity of light, while the cones, in addition, are impressed by qualitative differences or color.

Accessory Structures.-The muscles which move the eyeball are six in number-the superior and inferior recti, the external and internal recti, the superior and inferior oblique muscles. The four recti muscles, arising from the apex of the orbit, pass forward and are inserted into the sides of the sclerotic coat; the superior and inferior muscles rotate the eye around a horizontal axis; the external and internal rotate it around a vertical axis.

The superior oblique muscle, having the same origin, passes forward to the inner and upper angle of the orbital cavity, where its tendon passes through a cartilaginous pulley; it is then reflected backward and inserted into the sclerotic just behind the transverse diameter. Its function is to 
rotate the eycball in such a manner as to direct the pupil docunzeard and outward.

The inferior oblique muscle arises at the inner angle of the orbit, and then passes outward and backward, to be inserted into the sclerotic. Its function is to rotate the eyeball and to direct the pupil uproard and outzuard.

By the associated action of all these muscles, the eyeball is capable of performing all the varied and complex movements necessary for distinct vision.

The eyelids, bordered with short, stiff hairs, shade the eye and protect it from injury. On the posterior surface, just beneath the conjunctiva, are the Meibomian glands, which secrete an oily fluid; this covers the edge of the lids, and prevents the tears from flowing over the cheek.

The lacrymal glands are ovoid in shape, and are situated at the upper and outer part of the orbital cavity; they open by from six to eight ducts at the outer portion of the upper lids.

The tears, secreted by the lacrymal glands, are distributed over the cornea by the lids during the act of winking, and keep it moist and free from dust. The excess of tears passes into the lacrymal ducts, which begin by two minute orifices, one on each lid, at the inner canthus. They conduct the tears into the nasal duct, and so into the nose.

\section{THE SENSE OF HEARING.}

The ear, or organ of hearing, is lodged within the petrous portion of the temporal bone. It may be, for convenience of description, divided into three portions-viz :

I. The external ear.

2. The middle ear.

3. The internal ear or labyrinth.

The external ear consists of the pinna, or auricle, and the external auditory canal. The pinna consists of a thin layer of cartilage, presenting a series of elevations and depressions; it is attached by fibrous tissue to the outer bony edge of the auditory canal ; it is covered by a layer of integument continuous with that covering the side of the head. The general shape of the pinna is concave, and presents, little below the center, a deep depression-the concha. The external auditory canal extends from the concha inward for a distance of about $1 / 4$ inches. It is directed some- 
what forward and upward, passing over a convexity of bone, and then dips downward to its termination; it is composed of both bone and cartilage, and is lined by a reflection of the skin covering the pinna. At the external portion of the canal the skin contains a number of tubular glands, - the ceruminous glands, - which in their conformation resemble the perspiratory glands. They secrete the cerumen, or ear-wax.

The middle ear, or tympanum, is an irregularly shaped cavity hollowed out of the temporal bone and situated between the external ear and the labyrinth. It is narrow from side to side, but relatively long in its vertical and anteroposterior diameters; it is separated from the external auditory canal by a membrane-the membrana tympani; from the internal ear it is separated by an osseomembranous partition, which forms a common wall for both cavities. The middle ear communicates posteriorly with the mastoid cells; anteriorly with the nasopharynx, by means of the Eustachian tube. The interior of this cavity is lined by mucous membrane continuous with that lining the pharynx.

The membrana tympani is a thin, translucent, nearly circular membrane, measuring about $\frac{2}{5}$ of an inch in diameter, placed at the inner termination of the external auditory canal. The membrane is inclosed within a ring of bone, which in the fetal condition can be easily removed, but in the adult condition becomes consolidated with the surrounding bone. The membrana tympani consists primarily of a layer of fibrous tissue, arranged both circularly and radially, and forms the membrana propria; externally it is covered by a thin layer of skin continuous with that lining the auditory canal ; internally it is covered by a thin mucous membrane. The tympanic membrane is placed obliquely at the bottom of the auditory canal, inclining at an angle of forty-five degrees, being directed from behind and above downward and inward. On its external surface this membrane presents a funnel-shaped depression, the sides of which are somewhat convex.

The Ear Bones.-Running across the tympanic cavity and forming an irregular line of joined levers is a chain of bones which articulate with one another at their extremities. They are known as the malleus, incus, and stapes.

The form and position of these bones are shown in figure 34 .

The malleus consists of a head, neck, and handle, of which the latter is attached to the inner surface of the membrana tympani; the incus, or anvil bone presents a concave, articular surface, which receives the head of the malleus; the stapes, or stirrup bone, articulates externally with the long 
process of the incus, and internally, by its oval base, with the edges of the foramen ovale.

The tensor tympani muscle consists of a fleshy, tapering portion, $1 / 2$ of an inch in length, which terminates in a slender tendon; it arises from the cartilaginous portion of the Eustachian tube and the adjacent surface of the sphenoid bone. From this origin the muscle passes nearly horizontally

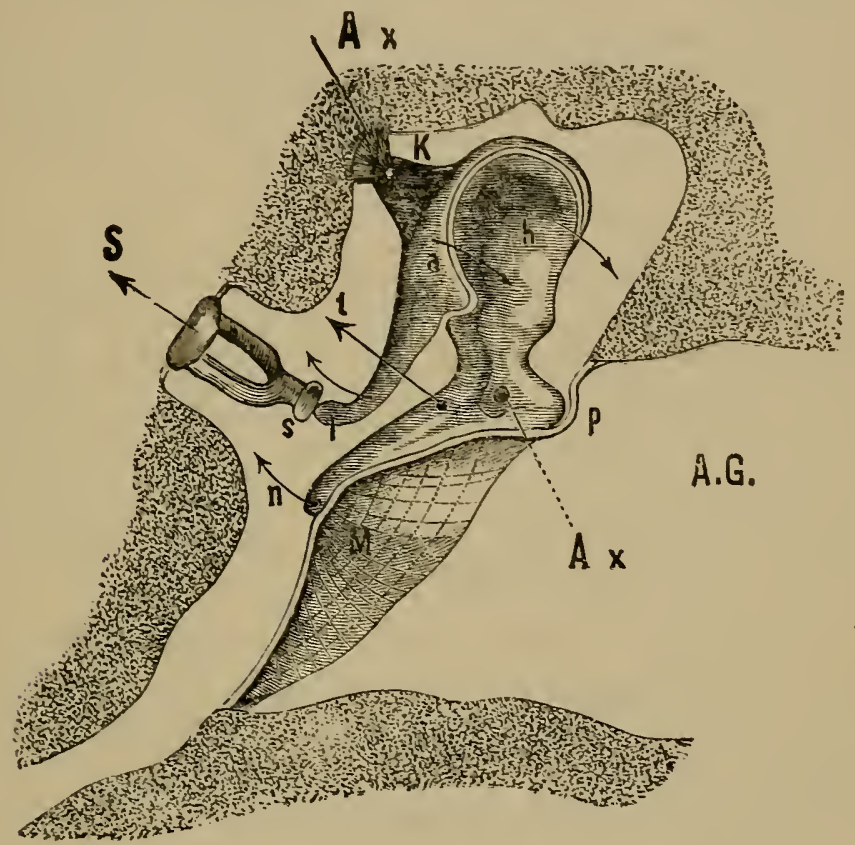

Fig. 34.-Tympanum and Auditory Ossicles (Left) Magnified.

A.G. External meatus. M. Membrana tympani, which is attached to the handle of the malleus, $n$, and near it the short process, $p$. h. Head of the malleus. a. Incus; $K$, its short process, with its ligament; 1 , long $p$ rocess. s. Sylvian ossicle. S. Stapes. Ax, Ax, is the axis of rotation of the ossicles; it is shown in perspective, and must be imagined to penetrate the plane of the paper. $t$. Line of traction of the tensor tympani. The other arrows indicate the movement of the ossicles when the tensor contracts.

backward to the tympanic cavity; just opposite to the fenestra ovalis its tendon bends at a right angle over the processus cochleariformis, and then passes outward across the cavity, to be inserted into the angle of the malleus near the neck.

The stapedius muscle emerges from the cavity of a pyramid of bone projecting from the posterior wall of the tympanum; the tendon passes 
forward, and is inserted into the neck of the stapes bone, posteriorly, near its point of articulation with the incus.

The laxator tympani muscle, so called, is now generally regarded as being ligamentous in nature, and not muscular.

The Eustachian tube, by means of which a free communication is established between the middle ear and the pharynx, is partly bony and partly cartilaginous in structure. It measures about $\mathbf{I} / 2$ inches in length ; commencing at its opening into the nasopharynx, it passes upward and outward to the spine of the sphenoid bone, at which point it becomes somewhat contracted; the tube then dilates as it passes backward into the middle-ear cavity; it is lined by mucous membrane, which is continued into the middle ear and mastoid cells.

The function of the ear, as a. whole, is the reception and transmission of aerrial vibrations to the terminal organs concealed within the internal ear, and which are connected with the auditory nerve-fibers. The excitation of these end organs caused by the impact of the vibrations arouses in the auditory nerve impulses which are then transmitted to the brain, where the hearing process takes place. In order to appreciate the functions of the individual parts of the ear, a few of the characteristics of sound waves must be kept in mind.

Sound Waves.-All sounds are caused by vibrations in the atmosphere which have been communicated to it by vibrating elastic bodies, such as membranes, strings, rods, etc. These vibrating bodies produce in the air a to-and-fro movement of its particles, resulting in a series of alternate condensations and rarefactions, which are propagated in all directions. A complete oscillation of a particle of air forward and backward constitutes a sound wave. Musical sounds are caused by a succession of regular waves, which follow one another with a certain rapidity. Noises are caused by the impact of a series of irregular waves.

All sound waves possess intensity, pitch, and quality. The intensity, or loudness, of a sound depends upon the amplitude of its vibrations or the extent of its excursion. The pitch depends upon the number of vibrations which affect the auditory nerve in a second of time; the pitch of the note $\mathrm{C}$, the first below the leger line of the musical scale, is caused by 256 vibrations a second; the pitch of the same note an octave higher is caused by $5 \mathrm{I} 2$ vibrations a second. If the vibrations are too few a second, they fail to be perceived as a continuous sound; the minimum number of vibrations capable of producing a sound has been fixed at sixteen a 
second; the highest pitched nusical note capable of being heard has been shown to be due to $3 \mathrm{~S}, 000$ vibrations a second. In the ascent of the musical scales there is, therefore, a gradual increase in the number of vibrations and a gradual increase in the pitch of the sounds. Between the two extreme limits lies the range of audibility, which embraces eleven octaves, of which seven are employed in the musical scale.

The quality of sound depends upon a combination of the fundamental vibration with certain secondary vibrations of subdivisions of the vibrating body. These so-called over-tones vary in intensity and pitch, and by modifying the form of the primary wave produce that which is termed the quality of sound.

Function of the Pinna and External Auditory Canal.-In those animals possessing movable ears the pinna plays an important part in the collection of sound wares. In man, in whom the capability of noving the pinna has been lost, it is doubtful if it is at all necessary for hearing. Nevertheless an individual with dull hearing may have the perception of sound increased by placing the pinna at an angle of 45 degrees to the side of the bead. The external aicitory canal transnits the sonorous vibrations to the tympanic membrane. Owing to the obliquity of this canal it has been supposed that the waves, concentrated at the concha, undergo a series of reflections on their way to the tympanic membrane, and, owing to the position of this membrane, strike it almost perpendicularly.

Function of the Tympanic Membrane.-The function of the tympanic membrane appears to be the reception of sound vibrations by being thrown by them into reciprocal vibrations which correspond in intensity and amplitude. That this membrane actually reproduces all vibrations within the range of audibility has been experimentally demonstrated. The membrane not being fixed, so far as its tension is concerned, does not possess a fixed fundamental note, like a stationary fixed membrane, and is, therefore, just as well adapted for the reception of one set of vibrations as for another. This is made possible by variations in its tension in accordance with the pitch of the sounds. In the absence of all sound the membrane is in a condition of relaxation; with the advent of sound waves possessing a gradual increase of pitch, as in the ascent of the music scale, the tension of the tympanic membrane is gradually increased until its maximum tension is reached at the upper limit of the range of audibility. By this change in tension certain tones become perceptible and distinct, while others become indistinct and inaudible.

Function of the Tensor Tympani Muscle.-The function of this 
muscle is, as its name indicates, to increase the tension of the membrane in accordance with the pitch of the sound wave. The tension of this muscle playing over the processus cochleariformis and attached at almost a right angle to the handle of the malleus will, when the muscle contracts, pull the handle inward, increase the convexity of the membrane, and at the same time increase its tension; with the relaxation of this muscle, the handle of the malleus passes outward and the tension is diminished. The contractions of the tensor muscle are reflex in character and excited by nerveimpulses reaching it through the small petrosal nerve and otic ganglion. The number of nerve stimuli passing to the muscle and determining the degree of contraction will depend upon the pitch of the sound wave and the subsequent excitation of the auditory nerve. The tensor tympani muscle may be regarded as an accommodative apparatus by which the tympanic membrane is so adjusted as to enable it to receive vibrations of varying degrees of pitch.

Function of the Ossicles.-The function of the chain of bones is to transmit the sound wave across the tympanic cavity to the internal ear. The first of these bones, the malleus, being attached to the tympanic membrane, will take up the vibrations much more readily than if no membrane intervened. Owing to the character of the articulations, when the handle of the malleus is drawn inward, the position of the bones is so changed that they form practically a solid rod, and are therefore much better adapted for the transmission of molecular vibrations than if the articulations remained loose. As the stapes bone is somewhat shorter than the malleus, its vibrations are slighter than those of the tympanic membrane, and by this arrangement the amplitude of the vibrations is diminished, but their force increased.

The function of the stapedius muscle is, according to Henle, to fix the stapes bone so as to prevent too great a movement from being communicated to it from the incus and transmitted to the perilymph. It may be looked upon, therefore, as a protective muscle.

The function of the Eustachian tube is to maintain a free communication between the cavity of the middle ear and the nasopharynx. The pressure of air within and without the ear is thus equalized, and the vibrations of the tympanic membrane are permitted to attain their maximum, one of the conditions essential for the reception of sound waves. The impairment in the acuteness of hearing which is caused by an unequal pressure of the air in the middle ear can be shown- 
1. By closing the mouth and nose and forcing air from the lungs through the Eustachian tube into the ear, producing an increase in pressure.

2. By closing the nose and mouth, and making efforts at deglutition, which withdraws the air from the ear and diminishes its pressure.

In both instances the free vibrations of the tympanic membrane are interfered with. The pharyngeal orifice of the Eustachian tube is opened by the action of certain of the muscles of deglutition-viz., the levator palati, the tensor palati, and the palato-pharyngei muscles.

The internal ear, or labyrinth, is located in the petrous portion of the temporal bone, and consists of an osseous and a membranous portion.

The osseous labyrinth is divisible into three parts-viz., the vestibule, the semicircular canals, and the cochlea.

The vestibule is a small, triangular cavity, which communicates with the middle ear by the foramen ovule; in the natural condition it is closed by the base of the stapes bone. The filaments of the auditory nerve enter the vestibule through small foramina in the inner wall, at the fovea hemispherica.

The semicircular canals are three in number, the superior vertical, the inferior vertical, and the horizontal, each of which opens into the cavity of the vestibule by two openings, with the exception of the two vertical, which at one extremity open by a common orifice.

The cochlea forms the anterior part of the internal ear. It is a gradually tapering canal, about $\mathbf{I} / 2$ inches in length, which winds spirally around a central axis, the modiolus, two and one half times. The interior of the cochlea is partly divided into two passages by a thin plate of bone, the lamina osseous spiralis, which projects from the central axis two thirds of the way across the canal. These passages are termed the scala vestibuli and the scala tympani, from their communication with the vestibule and tympanum. The scala tympani communicates with the middle ear through the furamen rotundum, which, in the natural condition, is closed by the second membrana tympani ; superiorly they are united by an opening, the helicotrema.

The whole interior of the labyrinth, the vestibule, the semicircular canals, and the scala of the cochlea, contains a clear, limpid fluid, the perilymph secreted by the periosteum lining the osseous walls.

The membranous labyrinth corresponds to the osseous labyrinth with respect to form, though it is somewhat smaller in size.

The vestibular portion consists of two small sacs, the utricle and the saccule. 
The semicircular canals communicate with the utricle in the same manner as the bony canals communicate with the vestibule. The saccule communicates with the membranous cochlea by the canalis reuniens. In the interior of the utricle and saccule, at the entrance of the auditory nerve, are small masses of carbonate of lime crystals, constituting the otoliths. Their function is unknown.

The membranous cochlea is a closed tube, commencing by a blind extremity at the first turn of the cochlea, and terminating at its apex by a blind extremity also. It is situated between the edge of the osseous lamina spiralis and the outer wall of the bony cochlea, and follows it in its turns around the modiolus.

A transverse section of the cochlea shows that it is divided into two portions by the osseous lamina and the basilar membrane :

I. The scala vestibuli, bounded by the periosteum and membrane of Reissner.

2. The scala tympani, occupying the inferior portion, and bounded above by the septum, composed of the osseous lamina and the membrana basilaris.

The true membranous canal is situated between the membrane of Reissner and the basilar membrane. It is triangular in shape, but is partly divided into a triangular portion and a quadrilateral portion by the tectorial membrane.

The organ of Corti is situated in the quadrilateral portion of the canal, and consists of pillars of rods of the consistence of cartilage. They are arranged in two rows-the one internal, the other external; these rods rest upon the basilar membrane; their bases are separated from one another, but their upper extremities are united, forming an arcade. In the internal row it is estimated there are about 3,500 and in the external row about 5,200 of these rods.

On the inner side of the internal row is a single layer of elongated haircells; on the outer surface of the external row are three such layers of haircells. Nothing definite is known as to their function.

The endolymph occupies the interior of the utricle, saccule, and membranous canals, and bathes the structures in the interior of the membranous cochlea throughout its entire extent.

The auditory nerve at the bottom of the internal auditory meatus divides into-

I. A vestibular branch, which is distributed to the utricle and to the semicircular canals. 
2. A cochlear branch, which passes into the central axis at its base and ascends to its apex; as it ascends, fibers are given off, which pass between the plates of the osseous lamina, to be ultimately connected with the organ of Corti.

The function of the semicircular canals appears to be to assist in maintaining the equilibrium of the body ; destruction of the vertical canal is followed by an oscillation of the head upward and downward; destruction of the horizontal canal is followed by oscillations from left to right. When the canals are injured on both sides, the animal loses the power of maintaining equilibrium upon making muscular movements.

Function of the Cochlea. - It is regarded as possessing the power of appreciating the quality of pitch and the shades of different musical tones. The elements of the organ of Corti are analogous, in some respects, to a musical instrument, and are supposed, by Helmholtz, to be tuned so as to vibrate in unison with the different tones conveyed to the internal ear.

Summary.-The waves of sound are gathered together by the pinna and external auditory meatus, and conveyed to the membrana tympani. This membrane, made tense or lax by the action of the tensor tympani and laxator tympani muscles, is enabled to receive sound waves of either high or low pitch. The vibrations are conducted across the middle ear by a chain of bones to the foramen ovale, and by the column of air of the tympanum to the foramen rotundum, which is closed by the second membrana tympani, the pressure of the air in the tympanum being regulated by the Eustachian tube.

The internal ear finally receives the vibrations, which excite vibrations successively in the perilymph, the walls of the membranous labyrinth, the endolymph, and, lastly, the terminal filaments of the auditory nerve, by which they are conveyed to the brain.

\section{VOICE AND SPEECH.}

The larynx is the organ of voice. Speech is a modification of voice, and is produced by the teeth and the muscles of the lips and tongue, coördinated in their action by stimuli derived from the cerebrum.

The structures entering into the formation of the larynx are mainly the thyroid, cricoid, and arytenoid cartilages; they are so situated and united 
by means of ligaments and muscles as to form a firm cartilaginous box. The larynx is covered externally by fibrous tissue, and lined internally with mucous membrane.

The vocal cords are four ligamentous bands, running anteroposteriorly across the upper portion of the larynx, and are divided into the two superior or false vocal cords, and the two inferior or true vocal cords; they are attached anteriorly to the receding angle of the thyroid cartilages, and posteriorly to the anterior part of the base of the arytenoid cartilages. The space between the true vocal cords is the rima glottidis.

The muscles which have a direct action upon the movements of the vocal cords are nine in number, and take their names from their points of origin and insertion-viz., the two crico-thyroid, two thyro-arytenoid, two posterior crico-arytenoid, two lateral crico arytenoid, and one arytenoid muscles.

The crico-thyroid muscles, by their contraction, render the vocal cords more tense by drawing down the anterior portion of the thyroid cartilage and approximating it to the cricoid, and at the same time tilting the posterior portion of the cricoid and arytenoid cartilages backward.

The thyro-arytenoid, by their contraction, relax the vocal cords by drawing the arytenoid cartilage forward and the thyroid backward.

The posterior crico-arytenoid muscles, by their contraction, rotate the arytenoid cartilages outward and thus separate the vocal cords and enlarge the aperture of the glottis. They principally aid the respiratory movements during inspiration.

The lateral crico-arytenoid muscles are antagonistic to the former, and by their contraction rotate the arytenoid cartilages so as to approximate the vocal cords and constrict the glottis.

The arytenoid muscle assists in the closure of the aperture of the glottis.

The inferior laryngeal nerve animates all the muscles of the larynx, with the exception of the crico-thyroid.

Movements of the Vocal Cords.-During respiration the movements of the vocal cords differ from those occurring during the production of voice.

At each inspiration the true vocal cords are widely separated, and the aperture of the glottis is enlarged by the action of the crico-arytenoid muscles, which rotate outward the anterior angle of the base of the arytenoid cartilages; at each expiration the larynx becomes passive; the elasticity of the vocal cords returns them to their original position, and the air is forced out by the elasticity of the lungs and the walls of the thorax. 
Phonation.-As soon as phonation is about to be accomplished, a marked change in the glottis is noticed with the aid of the laryngoscope. The true vocal cords suddenly become approximated and are made parallel, giving to the glottis the appearance of a narrow slit, the edges of which are capable of vibrating accurately and rapidly; at the same time their tension is much increased.

With the vocal cords thus prepared, the expiratory muscles force the columin of air into the lungs and trachea through the glottis, throwing the edges of the cords into vibration.

The pitch of sounds depends upon the extent to which the vocal cords are made tense and the length of the aperture through which the air passes. In the production of sounds of a high pitch, the tension of the vocal cords becomes very marked and the glottis diminished in length. When sounds having a low pitch are emitted from the larynx, the rocal cords are less tense and their vibrations are large and loose.

The quality of voice depends upon the length, size, and thickness of the cords, and upon the size, form, and construction of the trachea, the larynx, and the resonant cavities of the pharynx, nose, and mouth.

The compass of the voice comprehends from two to three octaves. The range is different in the two sexes, the lowest note of the male being about one octave lower than the lowest note of the female; while the highest note of the male is an octave less than the highest note of the female.

The varieties of voice-e. g., bass, baritone, tenor, contralto, mezzosoprano, and soprano-are due to the length of the vocal cords, being longer when the roice has a low pitch, and shorter when it has a high pitch.

Speech is the faculty of expressing ideas by means of combinations of sounds, in obedience to the dictates of the cerebrum.

Articulate sounds may be divided into vowrels and consonants. The rowe sounds, a, e, $i, o, u$, are produced in the larynx by the vocal cords. The consonant sounds are produced in the air-passages above the laryn $\mathrm{x}$ by an interruption of the current of air by the lips, tongue, and teeth ; the consonants may be divided into :

I. Mutes, $b, d, k, p, t,{ }^{\circ} c, g$.

2. Dentals, $d, j, s, t, z$.

3. Nasals, $m, n, n g$.

4. Labials, $b, p, f, v, m$.

5. Gutturals, $k, g, c$, and $g$ hard

6. Liquids, $l, m, n, r$. 


\section{EMBRYOLOGY.}

Reproduction is the function by which the species is preserved; it is accomplished by the organs of generation in the two sexes. Embryology is the science which investigates the successive stages in the development of the embryo.

\section{GENERATIVE ORGANS OF THE FEMALE.}

The generative organs of the female consist of the ovaries, Fallopian tubes, uterus, and vagina.

The ovaries are two small, ovoid, flattened bodies, measuring $I \frac{1}{2}$ inches in length and $3 / 4$ of an inch in width ; they are situated in the cavity of the pelvis, and are imbedded in the posterior layer of the broad ligament; attached to the uterus by a round ligament, and to the extremities of the Fallopian tubes by the fimbriæ. The ovary consists of an external membrane o fibrous tissue, the cortical portion, in which are embedded the Graafian z'esicles, and an internal portion, the stroma, containing blood-vessels.

The Graafian vesicles are exceedingly numerous, but are situated only in the cortical portion. Although the ovary contains the vesicles from the period of birth, it is only at puberty that they attain their full development. From this time onward to the catamenial period there is a constant growth and maturation of the Graafian vesicles. They consist of an external investment, composed of fibrous tissues and blood-vessels, in the interior of which is a layer of cells forming the membrana granulosa; at its lower portion there is an accumulation of cells, the proligerous disc, in which the orum is contained. The cavity of the vesicle contains a slightly yellowish alkaline, albuminous fluid.

The ovum is a globular body, measuring about $I^{\frac{1}{2} 5}$ of an inch in diameter : it consists of an external investing membrane, the vitelline membrane; a central granular substance, the vitellus, or yolk; a nucleus, the germinal vesicle, in the interior of which is imbedded the nucleolus, or germinal spot.

The Fallopian tubes are about four inches in length, and extend outward from the upper angles of the uterus, between the folds of the broad 
ligaments, and terminate in a fringed extremity which is attached by one of the fringes to the ovary. They consist of three coats :

I. The external, or peritoneal.

2. Middle, or muscular, the fibers of which are arranged in a circular or longitudinal direction.

3. Internal, or mucous, covered with ciliated epithelial cells, which are always waving from the ovary toward the uterus.

The uterus is pyriform in shape, and may be divided into a body and neck; it measures about three inches in length and two inches in breadth in the unimpregnated state. At the lower extremity of the neck is the os externum; at the junction of the neck with the body is a constriction, the os internum. The cavity of the uterus is triangular in shape, the walls of the triangle being almost in contact.

The walls of the uterus are made up of several layers of non-striated muscle-fibers, covered externally by peritoneum, and lined internally by mucous membrane, containing numerous tubular glands, and covered by ciliated epithelial cells.

The vagina is a membranous canal, from five to six inches in length, situated between the rectum and bladder. It extends obliquely upward from the surface, almost to the brim of the pelvis, and embraces at its upper extremity the neck of the uterus.

Discharge of the Ovum.-As the Graafian vesicle matures it increases in size, from an augmentation of its liquid contents, and approaches the surface of the ovary, where it forms a projection, measuring from $1 / 4$ to $1 / 2$ of an inch. The maturation of the vesicle occurs periodically, about every twenty-eight days, and is attended by the phenomena of menstruation. During this period of active congestion of the reproductive organs the Graafian vesicle ruptures, the ovum and liquid contents escape, and are caught by the fimbriated extremity of the Fallopian tube, which has adapted itself to the posterior surface of the ovary. The passage of the ovum through the Fallopian tube into the uterus occupies from ten to fourteen days, and is accomplished by muscular contraction and by the action of the ciliated epithelium.

Menstruation is a periodic discharge of blood from the mucous membrane of the uterus, due to a fatty degeneration of the small blood-vessels. Under the pressure of an increased amount of blood in the reproductive organs, attending the process of ovulation, the blood-vessels rupture, and a hemorrhage takes place into the uterine cavity; thence it passes into the 
vagina. Menstruation lasts from five to six days, and the amount of blood discharged averages about five ounces.

Corpus Luteum.-For some time previous to the rupture of a Graafian vesicle it increases in size and becomes vascular; its walls become thickened from the deposition of a reddish-yellow, glutinous substance, a product of cell growth from the proper coat of the follicle and the membrana granulosa. After the ovum escapes there is usually a small effusion of blood into the cavity of the follicle, which soon coagulates, loses its coloring-matter, and acquires the characteristics of fibrin, but it takes no part in the formation of the corpus luteum. The walls of the follicle become convoluted and vascular, and undergo hypertrophy, until they occupy the whole of the follicular cavity. At its period of fullest development the corpus luteum measures $3 / 4$ of an inch in length and $1 / 2$ of an inch in depth. In a few weeks the mass loses its red color and becomes yellow, constituting the corpus luteum, or yellow body. It then begins to retract and becomes pale; and at the end of two months nothing remains but a small cicatrix upon the surface of the ovary. Such are the changes in the follicle if the ovum has not been impregnated.

The corpus luteum, after impregnation has taken place, undergoes a much slower development, becomes larger, and continues during the entire period of gestation. The difference between the corpus luteum of the unimpregnated and pregnant condition is expressed in the following table by Dalton :

\section{Corpus Luteum of Menstruation. Corpus Luteum of Pregnancy.}

At the end of Three quarters of an inch in diameter; central clot redthree weeks. One month.

Two months. dish; convoluted wall pale.

Smaller; convoluted I arger ; convoluted wall wall bright yellow; clot bright yellow; clot still reddish. still reddish.

Reduced to the condi-
tion of an insignificant cicatrix.

Four months. Absent or unnoticeable.

Redush.

Seven eighths of an inch in diameter; convoluted wall bright yellow; clot perfectly decolorized.

\begin{tabular}{l|l|l} 
Six months. & $\begin{array}{l}\text { Able. } \\
\text { Nine months. }\end{array}$ Absent. & $\begin{array}{l}\text { diameter; clot pale and fibrinous; } \\
\text { convoluted wall dull yellow. } \\
\text { Still as large as at the end of } \\
\text { second month; clot fibrinous; } \\
\text { convoluted wall paler. } \\
\text { Half an inch in diameter ; cen- } \\
\text { tral clot converted into a radiat- } \\
\text { ing cicatrix ; external wall toler- } \\
\text { ably thick and convoluted, but } \\
\text { without any bright yellow color. }\end{array}$
\end{tabular}




\section{GENERATIVE ORGANS OF THE MALE.}

The generative organs of the male consist of the testicles, vasa deferentia, vesiculi seminales, and penis.

The testicles, the essential organs of reproduction in the male, are two oblong glands, about $\mathbf{I} 1 / 2$ inches in length, compressed from side to side, and situated in the cavity of the scrotum.

The proper coat of the testicle, the tunica albuginea, is a white, fibrous structure, about $\frac{1}{25}$ of an inch in thickness; after enveloping the testicle, it is reflected into its interior at the posterior border, and forms a vertical process, the mediastinum testis, from which septa are given off, dividing the testicle into lobules.

The substance of the testicle is made up of the seminiferous tubules, which exist to the number of 840 ; they are exceedingly convoluted, and when unravelled are about thirty inches in length. As they pass toward the apices of the lobules, they become less convoluted, and terminate in from twenty to thirty straight ducts, the vasa recta, which pass upward through the mediastinum and constitute the rete testis. At the upper part of the mediastinum the lobules unite to form from nine to thirty small ducts, the vasa efferentia, which become convoluted and form the globus major of the epididymis; the continuation of the tubes downward behind the testicle and a second convolution constitutes the body and globus minor.

The seminal tubule consists of a basement membrane lined by granular nucleated epithelium.

The vas deferens, the excretory duct of the testicle, is about two feet in length, and may be traced upward from the epididymis to the under surface of the base of the bladder, where it unites with the duct of the vesicula seminalis to form the ejaculatory duct.

The vesiculæ seminales are two lobulated, pyriform bodies about two inches in length, situated on the inner surface of the bladder.

They have an external fibrous coat, a middle muscular coat, and an internal mucous coat, covered by epithelium, which secretes a mucous fluid. The vesiculæ seminales serve as reservoirs, in which the seminal fluid is temporarily stored up.

The ejaculatory duct, about $3 / 4$ of an inch in length, opens into the urethra, and is formed by the union of the vasa deferentia and the ducts of the vesiculæ seminales.

The prostate gland surrounds the posterior extremity of the urethra, 
and opens into it by from twenty to thirty openings, the orifices of the prostatic tubules. The gland secretes a fluid which forms part of the semen and assists in maintaining the vitality of the spermatozoa.

Semen is a complex fluid, made up of the secretions from the testicles, the vesiculæ seminales, the prostatic and urethral glands. It is grayishwhite in color, mucilaginous in consistence, of a characteristic odor, and somewhat heavier than water. From half a dram to a dram is ejaculated at each orgasm.

The spermatozoa are peculiar anatomic elements, developed within the seminal tubules, and possess the power of spontaneous movement. The spermatozoa consist of a conoid head and a long, filamentous tail, which is in continuous and active motion; so long as they remain in the vas deferens they are quiescent, but when free to move in the fluid of the vesiculæ seminales, they become very active.

Origin.-The spermatozoa appear at the age of puberty, and are then constantly formed until an advanced age. They are developed from the nuclei of large, round cells contained in the anterior of the seminal tubules, as many as fifteen to twenty developing in a single cell.

When the spermatozoa are introduced into the vagina, they pass readily into the uterus and through the Fallopian tubes toward the ovaries, where they remain and retain their vitality for a period of from eight to ten days.

Fecundation is the union of the spermatozoa with the ovum during its passage toward the uterus, and usually takes place in the Fallopian tube, just outside of the womb. After floating around the ovum in an active manner, they penetrate the vitelline membrane, pass into the interior of the vitellus, where they lose their vitality, and, along with the germinal vesicle, entirely disappear.

\section{DEVELOPMENT OF ACCESSORY STRUCTURES.}

Segmentation of the Vitellus.-After the disappearance of the spermatozoa and the germinal vesicle there remains a transparent, granular, albuminous substance, in the center of which a new nucleus soon appears; this constitutes the parent cells, and is the first stage in the development of the new being.

Following this, the vitellus undergoes segmentation; a constriction appears on the opposite side of the vitellus, which gradually deepens, until the yolk is divided into two segments, each of which has a distinct nucleus and 
nucleolus; these two segments undergo a further division into four, the four into eight, the eight into others, and so on, until the entire vitellus is divided into a great number of cells, each of which contains a nucleus and a nucleolus.

The peripheral cells of this "mulberry mass" then arrange themselves so as to form a membrane, and, as they are subjected to mutual pressure, assume a polyhedral shape, which gives to the membrane a mosaic appearance. The central part of the vitellus becomes filled with a clear fluid. A second membrane shortly appears within the first, and the two together constitute the external and internal blastodermic membranes.

Germinal Area.-At about this period there is an accumulation of cells at a certain spot upon the surface of the blastodermic membranes, which marks the position of the future embryo. This spot, at first circular, soon becomes elongated, and forms the primitive trace, around which is a clear space, the area pellucida, which is itself surrounded by a darker region, the area opaca.

The primitive trace soon disappears, and the area pellucida becomes guitar-shaped ; a new groove, the medullary groove, is now formed, which develops from before backward, and becomes the neural canal.

Blastodermic Membranes.-The embryo, at this period, consists of three layers-viz., the external and the internal blastodermic membranes and a middle membrane formed by a genesis of cells from their internal surfaces. These layers are known as the epiblast, mesoblast, and hypoblast.

The epiblast gives rise to the central nervous system, the epidermis of the skin and its appendages, and the primitive kidneys.

The mesoblast gives rise to the dermis, muscles, bones, nerves, bloodvessels, sympathetic nervous system, connective tissue, the urinary and reproductive apparatus, and the walls of the alimentary canal.

The hypoblast gives rise to the epithelial lining of the alimentary canal and its glandular appendages, the liver and pancreas, and the epithelium of the respiratory tract.

Dorsal Laminæ.-As development advances, the true medullary groove deepens, and there arise two longitudinal elevations of the epiblast, -the dorsal lamina, one on either side of the groove,- which grow up, arch over, and unite so as to form a closed tube, the primitive central nervous system.

The chorda dorsalis is a cylindric rod running almost throughout the entire length of the embryo. It is formed by an aggregation of mesoblastic cells, and is situated immediately beneath the medullary groove. 
Primitive Vertebræ.- - On either side of the neural canal the cells of the mesoblast undergo a longitudinal thickening, which develops and extends around the neural canal and the chorda dorsalis, and forms the arches and bodies of the vertebre. They become divided transversely into foursided segments.

The mesoblast now separates into two layers: the external, joining with the epiblast, forms the somatopleura; the internal, joining with the hypoblast, forms the splanchnopleura; the space between them constitutes the pleuro-peritoneal cavity.

Visceral Laminæ. - The walls of the pleuro-peritoneal cavity are formed by a downward prolongation of the somatopleura (the visceral lamina), which, as they extend around in front, pinch off a portion of the yolk-sac (formed by the splanchnopleura), which becomes the primitive alimentary canal ; the lower portion, remaining outside of the body cavity, forms the umbilical vesicle, which after a time disappears.

Formation of Fetal Membranes. - The amnion appears shortly after the embryo begins to develop, and is formed by folds of the epiblast and external layer of the mesoblast, rising up in front and behind and on each side; these amniotic folds gradually extend over the back of the embryo to a certain point, where they coalesce and inclose a cavity-the amniotic cavity. The membranous partition between the folds disappears, and the outer layer recedes and becomes blended with the vitelline membrane, constituting the chorion-the external covering of the embryo.

The Allantois. - As the amnion develops, there grows out from the posterior portion of the alimentary canal a pouch, or diverticulum (the allantois), which carries blood-vessels derived from the intestinal circulation. As it gradually enlarges it becomes more vascular, and inserts itself between the two layers of the amnion, coming into intimate contact with the external layer. Finally, from increased growth, it completely surrounds the embryo, and its edges become fused together.

In the bird the allantois is a respiratory organ, absorbing oxygen and exhaling carbonic acid; it also absorbs nutritive matter from the interior of the egg.

Amniotic Fluid.-The amnion, when first formed, is in close contact with the surface of the ovum; but it soon enlarges, and becomes filled with a clear, transparent fluid, containing albumin, glucose, fatty matters, urea, and inorganic salts. It increases in amount up to the latter period of gestation, when it amounts to about two pints. In the space between 
the amnion and allantois is a gelatinous material, which is encroached upon and finally disappears as the amnion and allantois come in contact, at about the fifth month.

The chorion, the external investment of the embryo, is formed by a fusion of the original vitelline membrane, the external layer of the amnion, and the allantois. The external surface now becomes covered with villous processes, which increase in number and size by the continual budding and growth of club-shaped processes from the main stem, and give to the chorion a shaggy appearance. They consist of a homogeneous granular matter, and are penetrated by branches of the blood-vessels derived from the aorta.

The presence of villous processes in the uterine cavity is proof positive of the previous existence of a fetus. They are characteristic of the chorion, and are found under no other circumstances.

At about the end of the second month the villosities begin to atrophy and disappear from the surface of the chorion, with the exception of those situated at the points of entrance of the fetal blood-vessels, which occupy about one third of its surface, where they continue to grow longer, become more vascular, and ultimately assist in the formation of the placenta; the remaining two thirds of the surface loses its villi and blood-vessels and becomes a simple membrane.

The umbilical cord connects the fetus with that portion of the chorion which forms the fetal side of the placenta. It is a process of the allantois, and contains two arteries and a vein, which have a more or less spiral direction. It appears at the end of the first month, and gradually increases in length until, at the end of gestation, it measures about twenty inches. The cord is also surrounded by a process of the amnion.

Development of the Decidual Membrane. - The interior of the uterus is lined by a thin, delicate mucous membrane, in which are embedded immense numbers of tubules, terminating in blind extremities-the uterine tubules. At each period of menstruation the mucous membrane becomes thickened and vascular, which condition, however, disappears after the usual menstrual discharge. When the ovum becomes fecundated, the mucous membrane takes on an increased growth, becomes more hypertrophied and vascular, sends up little processes or elevations from its surface, and constitutes the decidua verd.

As the ovum passes from the Fallopian tube into the interior of the uterus, the primitive vitelline membrane, covered with villosities, becomes entangled with the processes of the mucous membrane. A portion of the 
decidua vera then grows up on all sides and incloses the ovum, forming the decidua reflexa, while the villous processes of the chorion insert themselves into the uterine tubules and in the mucous membrane between them.

As development advances, the decidua reflexa increases in size, and at about the end of the fourth month comes in contact with the decidua vera, with which it is ultimately fused.

The Placenta.-Of all the embryonic structures, the placenta is the most important. It is formed in the third month, and then increases in size until the seventh month, when a retrogressive metamorphosis takes place until its separation during labor, at which time it is of an oval or rounded shape, and measures from seven to nine inches in length, six to eight inches in breadth, and weighs from fifteen to twenty ounces. It is most frequently situated at the upper and posterior part of the inner surface of the uterus.

The placenta consists of two portions, a fetal and a maternal.

The fetal portion is formed by the villi of the chorion, which, by developing, rapidly increase in size and number. They become branched and penetrate the uterine tubules, which enlarge and receive their many ramifications. The capillary blood-vessels in the anterior of the villi also enlarge and freely anastomose with one another.

The maternal portion is formed from that part of the hypertrophied and vascular decidual membrane between the ovum and the uterus, the decidua serotina. As the placenta increases in size, the maternal blood-vessels around the tubules become more and more numerous, and gradually fuse together, forming great lakes, which constitute sinuses in the walls of the uterus.

As the terminal period of gestation approaches, the villi extend deeper into the decidua, while the sinuses in the maternal portion become larger and extend further into the chorion. Finally, from excessive development of the blood-vessels, the structures between them disappear, and as their walls come in contact they fuse together, so that, ultimately, the maternal and fetal blood are separated only by a thin layer of a homogeneous substance. When fully formed, the placenta consists principally of blood-vessels interlacing in every direction. The blood of the mother passes from the uterine vessels into the lake surrounding the villi; the blood from the fetus flows from the umbilical arteries into the interior of the villi; but there is not at any time an intermingling of blood, the two being separated by a delicate membrane formed by a fusion of the walls of the blood-vessels and the walls of the villi and uterine sinuses. 
The function of the placenta, besides nutrition, is that of a respiratory organ, permitting the oxygen of the maternal blood to pass by osmosis through the delicate placental membrane into the blood of the fetus; at the same time permitting the carbonic acid and other waste products, the result of nutritive changes in the fetus, to pass into the maternal blood, and so to be carried to the various eliminating organs.

Through the placenta also passes all the nutritious materials of the maternal blood which are essential to the development of the embryo.

At about the middle of gestation there develops beneath the decidual membrane a new mucous membrane, destined to perform the functions of the old when it is extruded from the womb, along with the other embryonic structures, during parturition.

\section{DEVELOPMENT OF THE EMBRYO.}

Nervous System.-The cerebro-spinal axis is formed within the medullary canal by the development of cells from its inner surfaces, which, as they increase, fill up the canal, and there remains only the central canal of the cord. The external surface gives rise to the dura mater and pia mater. The neural canal thus formed is a tubular membrane ; it terminates posteriorly in an oval dilatation, and anteriorly in a bulbous extremity, which soon becomes partially contracted, and forms the anterior, middle, and posterior cerebral vesicles, from which are ultimately developed the cerebrum, the corpora quadrigemina, and the medulla oblongata, respectively.

The anterior vesicle soon subdivides into two secondary vesicles, the larger of which becomes the hemispheres, the smaller the optic thalami; the posterior vesicle also divides into two, the anterior becoming the cerebellum, the posterior the pons Varolii and medulla oblongata.

About the seventh week the straight chain of cerebral vesicles becomes curved from behind forward and forms three prominent angles. As development advances, the relative size of the encephalic masses changes. The cerebrum, developing more rapidly than the posterior portion of the brain, soon grows backward and arches over the optic thalami and the tubercula quadrigemina; the cerebellum overlaps the medulla oblongata.

The surface of the cerebral hemispheres is at first smooth, but at about the fourth month begins to be marked by the future fissures and convolutions.

The eye is formed by a little bud projecting from the side of the anterior vesicle. It is at first hollow, but becomes lined with nervous matter, form- 
ing the optic nerve and retina; the remainder of the cavity is occupied by the vitreous body. The anterior portion of the pouch becomes invaginated and receives the crystalline lens, which is a product of the epiblast, as is also the cornea. The iris appears as a circular membrane without a central aperture, about the seventh week; the eyelids are formed between the second and third months.

The internal ear is developed from the auditory vesicle, budding from the third cerebral vesicle; the membranous vestibule appears first, and from it diverticula are given off, which become the semicircular canals and the cochlea.

The cavity of the tympanum, the Eustachian tube, and the external auditory canal are the remains of the first branchial cleft, the cavity of this cleft being subdivided into the tympanum and external auditory meatus by the membrana tympani.

The Skeleton.-The chorda dorsalis, the primitive part of the vertebral column, is a cartilaginous rod situated beneath the medullary groove. It is a temporary structure, and disappears as the true bony vertebræ develop. On either side are the quadrate masses of the mesoblast, the primitive vertebræ, which send processes upward and around the medullary groove, and downward and around the chorda dorsalis, forming in these situations the arches and bodies of the future vertebræ.

More externally the outer layers of the mesoblast and epiblast arch downward and forward, forming the ventral laminæ, in which develop the muscles and bones of the abdominal walls.

The true cranium is an anterior development of the vertebral column, and consists of the occipital, parietal, and frontal segments, which correspond to the three cerebral vesicles. The base of the cranium consists, at this period, of a cartilaginous rod on either side of the anterior extremity of the chorda dorsalis, in which three centers of ossification appear, the basi-occipital, the basisphenoid, and the presphenoid. They ultimately develop into the basilar process of the occipital bone and the body of the sphenoid.

The entire skeleton is at first either membranous or cartilaginous. At the beginning of the second month centers of ossification appear in the jaws and clavicle; as development advances the ossific points in all the future bones extend, until ossification is completed.

The limbs develop from four little buds projecting from the sides of the embryo, which, as they increase in length, separate into the thigh, leg, and 
foot, and the arm, forearm, and hand; the extremities of the limbs undergo subdivision, to form the fingers and toes.

Face and Visceral Arches.-In the facial and cervical regions the visceral laminæ send up three processes, the visceral arihes, separated by clefts, the visceral clefts.

The first, or the mandibular arches, unite in the median line to form the lower jaw, and superiorly form the malleus. A process jutting from its base grows forward, unites with the frontonasal process growing from above, and forms the upper jaw. When the superior maxillary processes fail to unite there results the cleft-palate deformity; if the integument also fails to unite, there results the hare-lip deformity. The space above the mandibular arch becomes the mouth.

The second arch develops the incus and stapes bones, the styloid process and ligament, and the lesser cornu of the hyoid bone. The cleft between the first and second arches partially closes up, but there remains an opening at the side, which becomes the Eustachian tube, tympanic cavity, and external auditory meatus.

The third arch develops the body and greater cornu of the hyoid bone.

Alimentary Canal and Its Appendages.-The alimentary canal is formed by a pinching-off of the yolk-sac by the visceral plates as they grow downward and forward. It consists of three distinct portions-the fore gut, the hind gut, and the central part, which communicates for some time with the yolk-sac. It is at first a straight tube, closed at both extremities, lying just beneath the vertebral column. The canal gradually increases in length and becomes more or less convoluted; at its anterior portion two pouches appear, which become the cardiac and pyloric extremities of the stomach, At about the seventh week the inferior extremity of the intestine is brought into communication with the exterior by an opening, the anus. Anteriorly the mouth and pharynx are formed by an involution of epiblast, which deepens until it communicates with the fore gut.

Th liver appears as a slight protrusion from the sides of the alimentary canal, about the end of the first month; it grows very rapidly, attains a large size, and almost fills up the abdominal cavity. The hepatic cells are derived from the intestinal epithelium, the vessels and connective tissue from the mesoblast.

The pancreas is formed by the hypoblastic membrane. It originates in two small ducts budding from the duodenum, which divide and subdivide, and develop the glandular structure.

The lungs are developed from the anterior part of the esophagus. At 
first a small bud appears, which, as it lengthens, divides into two branches ; secondary and tertiary processes are given off from these, which form the bronchial tubes and air-cells. The lungs originally extended into the abdominal cavity, but became confined to the thorax by the development of the diaphragm.

The bladder is formed by a dilatation of that portion of the allantois remaining within the abdominal cavity. It is at first pear-shaped and communicates with the intestine, but later becomes separated and opens exteriorly by the urethra. It is attached to the abdominal walls by a rounded cord-the urachus, the remains of a portion of the allantois.

Genito-urinary Apparatus.-The Wolffian bodies appear about the thirteenth day, as long, hollow tubes running along each side of the primitive vertebral column. They are temporary structures, and are sometimes called the primordial kidneys. The Wolffian bodies consist of tubules which run transversely and are lined with epithelium; internally they become invaginated to receive tufts of blood-vessels; externally they open into a common excretory duct, the duct of the Wolffian body, which unites with the duct of the opposite body and empties into the intestinal canal at a point opposite the allantois. On the outer side of the Wolffian body there appears another duct, the duct of Müller, which also opens into the intestine.

Behind the Wolffian bodies are developed the structures which become either the ovaries or testicles. In the development of the female the Wolffian bodies and their ducts disappear; the extremities of the Müllerian ducts dilate and form the fimbriated extremity of the Fallopian tubes, while the lower portions coalesce to form the body of the uterus and vagina, which now separate themselves from the intestine.

In the development of the male the Müllerian ducts atrophy, and the ducts of the Wolffian body ultimately form the epididymis and vas deferens. About the seventh month the testicles begin to descend, and by the ninth month have passed through the abdominal ring into the scrotum.

The kidneys are developed out of the Wolffian bodies. They consist of little pyramidal lobules, composed of tubules which open at the apex into the pelvis. As they pass outward they become convoluted and cup-shaped at their extremities, receive a tuft of blood-vessels, and form the Malpighian bodies.

The ureters are developed from the kidneys and pass downward to be connected with the bladder.

The circulatory apparatus assumes three forms at different periods of 
life, all having reference to the manner in which the embryo receives nutritive matter and is freed of waste products.

The vitelline circulation appears first and absorbs nutritive material from the vitellus. It is formed by blood-vessels which emerge from the body and ramify over a portion of the vitelline membrane, constituting the area vasculosa. The heart, lying in the median line, gives off two arches, which unite to form the abdominal aorta, from which two large arteries are given off, passing into the vascular area ; the venous blood is returned by veins which enter the heart. These vessels are known as the omphalomesenteric arteries and veins. The vitelline circulation is of short duration in mammals, as the supply of nutritive matter in the vitellus soon becomes exhausted.

The placental circulation becomes established when the blood-vessels in the allantois enter the villous processes of the chorion and come into close relationship with the maternal blood-vessels. The circulation lasts during the whole of intra-uterine life, but gives way at birth to the adult circulation, the change being made possible by the development of the circulatory apparatus.

The heart appears as a mass of cells coming off from the anterior portion of the intestine; its central part liquefies, and pulsations soon begin. The heart is at first tubular, receiving posteriorly the venous trunks and giving off anteriorly the arterial trunks. It soon becomes twisted upon itself, so that the two extremities lie upon the same plane.

The heart now consists of a single auricle and a single ventricle. A septum, growing from the apex of the ventricle, divides into two cavities, a right and a left. The auricles also become partly separated by a septum, which is perforated by the foramen ovale. The arterial trunk becomes separated, by a partition, into two canals, which become, ultimately, the aorta and the pulmonary artery. The auricles are separated from the ventricles by incomplete septa, through which the blood passes into the ventricles.

Arteries. - The aorta arises from the cephalic extremity of the heart and divides into two branches, which ascend, one on each side of the intestine, and unite posteriorly to form the main aorta ; posteriorly to these first aortic arches four others are developed, so that there are five altogether running along the visceral arches. The two anterior soon disappear. The third arch becomes the internal carotid and the external carotid; a part of the fourth arch, on the right side, becomes the subclavian artery, and the remainder atrophies and disappears, but on the left side it enlatges and becomes the permanent aorta ; the fifth arch becomes the pulmonary artery 
on the left side. The communication between the pulmonary artery and the aorta, the ductus arteriosus, disappears at an early period.

Veins.-The venous system appears first as two short, transverse veins, the canals of Cuvier, formed by the union of the vertebral veins and the cardinal veins, which empty into the auricle. The inferior vena cava is formed, as the kidneys develop, by the union of the renal veins, which, in a short time, receive branches from the lower extremities. The subclavian veins join the jugular as the upper extremities develop. The heart descends in the thorax, and the canals of Cuvier become oblique; they shortly communicate by a transverse duct, which ultimately becomes the left innominate vein. The left canal of Cuvier atrophies and becomes a fibrous cord. A transverse branch now appears, which carries the blood from the left cardiac vein into the right, and becomes the vena azygos minor; the right cardiac vein becomes the vena azygos major.

Circulation of Blood in the Fetus.--The blood returning from the placenta, after having received oxygen and being freed from carbonic acid, is carried by the umbilical vein to the under surface of the liver; here a portion of it passes through the ductus venosus into the ascending vena cava, while the remainder flows through the liver and passes into the vena cava by the hepatic veins. When the blood is emptied into the right auricle, it is directed by the Eustachian valve through the foramen ovale, into the left auricle, thence into the left ventricle, and so into the aorta and to all parts of the system. The venous blood returning from the head and upper extrenities is emptied, by the superior vena cava, into the right auricle, from which it passes into the right ventricle, and thence into the pulmonary artery. Owing to the condition of the lung only a small portion flows through the pulmonary capillaries, the greater part passing through the ductus arteriosus, which opens into the aorta at a point below the origin of the carotid and subclavian arteries. The mixed blood now passes down the aorta to supply the lower extremities, but a portion of it is directed, by the hypogastric arteries, to the placenta, to be again oxygenated.

At birth, the placental circulation gives way to the circulation of the adult. As soon as the child begins to breathe, the lungs expand, blood flows freely through the pulmonary capillaries, and the ductus arteriosus begins to contract. The foramen ovale closes about the tenth day. The umbilical vein, the ductus venosus, and the hypogastric arteries become impervious in several days, and ultimately form rounded cords. 


\section{TABLE OF PHYSIOLOGIC CONSTANTS.}

Mean height of male, 5 feet $61 / 2$ inches ; of female, 5 feet 2 inches.

Mean weight of male, 145 pounds; of female, I2I pounds.

Number of chemic elements in the human body : from 16 to 18 .

Number of proximate principles in the human body : about 100 .

Amount of water in the body weighing 145 pounds : 109 pounds.

Amount of solids in the body weighing 145 pounds : $3^{6}$ pounds.

Amount of saliva secreted in 24 hours : about $3 \frac{1}{2}$ pounds.

Function of saliva : converts starch into maltose.

Active principle of saliva : ptyalin.

Amount of gastric juice secreted in 24 hours : from 8 to I 4 pounds.

Function of gastric juice : converts albumin into peptone.

Active principles of gastric juice : pepsin and hydrochloric acid. Duration of digestion : from 3 to 5 hours.

Amount of intestinal juice secreted in 24 hours : about I pound.

Function of intestinal juice : converts cane sugar into dextrose and levulose ; maltose into dextrose.

Amount of pancreatic juice secreted in 24 hours : about $\mathrm{I} / 2$ pounds.

Active principles of pancreatic juice : trypsin, amylopsin, and steapsin.

Functions: $\left\{\begin{array}{l}\text { I. Splits the neutral fats into fatty acids and glycerin. } \\ \text { 2. Converts albumin into peptone. } \\ \text { 3. Converts starch into maltose. }\end{array}\right.$

Amount of bile poured into the intestines daily : about $21 / 2$ pounds.

Functions: $\left\{\begin{array}{l}\text { I. Assists in the emulsification of fats. } \\ \text { 2. Stimulates the peristaltic movements. } \\ \text { 3. Prevents putrefactive changes in the food. } \\ \text { 4. Promotes the absorption of fat. }\end{array}\right.$

Amount of blood in the body : from 16 to 18 pounds.

Size of red corpuscles : $\frac{1}{3} \frac{1}{2} \overline{0}$ of an inch.

Size of white corpuscles: $\frac{1}{250}$ of an inch.

Shape of red corpuscles : circular biconcave discs.

Shape of white corpuscles: globular.

Number of red corpuscles in a cubic millimeter of blood (the cubic $\frac{1}{25}$ of an inch) : 5,000,000. 
Function of red corpuscles : to carry oxygen from the lungs to the tissues.

Frequency of the heart's pulsation a minute : 72 on the average.

Velocity of the blood movement in the arteries: about 12 inches a second.

Length of time required for the blood to make an entire circuit of the vas-

cular system : about 20 seconds.

Amount of air passing in and out of the lungs at each respiratory act : from 20 to 30 cubic inches.

Amount of air that can be taken into the lungs on a forced inspiration : I 10 cubic inches.

Amount of reserve air in the lungs after an ordinary expiration : roo cubic inches.

Amount of residual air always remaining in the lungs : about roo cubic inches.

Vital capacity of the lungs : 230 cubic inches.

Entire volume of air passing in and out of the lungs in 24 hours : about 400 cubic feet.

Composition of the air: nitrogen, 79.19: oxygen, 20.81, in roo parts.

Amount of oxygen absorbed in 24 hours : 18 cubic feet.

Amount of carbonic acid exhaled in 24 hours : 14 cubic feet.

Temperature of the human body at the surface $: 98_{10}^{6} \mathrm{~F}$.

Amount of urine excreted daily : from 40 to 50 ounces.

Amount of urea excreted daily : 512 grains.

Specific gravity of urine : from ror 5 to 1025 .

Number of spinal nerves : $3^{\mathrm{I}}$ pairs.

Number of roots of origin : two; Ist, anterior, efferent; $2 \mathrm{~d}$, posterior, afferent.

Rate of transmission of nerve force : about roo feet a second.

Number of cranial nerves : 12 pairs.
Nerves of special sense:
I. Olfactory, or first pair.
2. Optic, or second pair.
3. Auditory, or eighth pair.
Nerves of special sense:
4. Chorda tympani for anterior two thirds of tongue.
5. Branches of glasso-pharyngeal, or eighth

Motor nerves to eyeball and accessory structures: motor oculi, or third pair; pathetic, or fourth pair; abducens, or sixth pair.

Motor nerves to facial muscles : portio dura, facial, or seventh pair.

Motor nerve to tongue : hypoglossal, or twelfth pair. 
Motor nerve to laryngeal muscles : spinal accessory, or eleventh pair.

Sensory nerve of the face : trifacial, or fifth pair.

Sensory nerve of the pharynx: glosso-pharyngeal, or ninth pair.

Sensory nerres of the lungs, stomach, etc.: pneumogastric, or tenth pair.

Length of spinal cord: 16 to 18 inches; weight, I $1 / 2$ ounces.

Point of decussation of motor fibers : at the medulla oblongata.

Point of decussation of sensory fibers: throughout the spinal cord.

Function of anterolateral column of spinal cord: transmit motor impulses from the brain to the muscles.

Function of the posterior columns : assist in the coördination of muscular morements.

Function of the medulla oblongata : controls the functions of insalivation, mastication, deglutition, respiration, circulation, etc.

Function of the cerebellum: center for the coördination of muscular morement.

Function of the cerebrum : center for intelligence, reason, and will.

Center for articulate language : third frontal convolution on the left side of cerebrum.

Number of coats to the eye : three : 1 st, cornea and sclerotic; 2 d, choroid ; $3 \mathrm{~d}$, retina.

Function of iris : regulates the amount of light entering the eye.

Function of crystalline lens : refracts the rays of light so as to form an image on the retina.

Function of retina : receives the impressions of light.

Function of membrana tympani : receives and transmits waves of sound to internal ear.

Function of Eustachian tube : regulates the passage of air into and from the middle ear.

Function of semicircular canals : assist in maintaining the equipoise of the body.

Function of the cochlea: appreciates the shades and combinations of musical tones.

Size of human ovum : $\frac{1}{125}$ of an inch in diameter.

Size of spermatozoa : $\frac{1}{4000}$ of an inch in length.

Function of the placenta: acts as a respiratory and digestive organ for the fetus.

Duration of pregnancy : 280 days.

I 8 
TABLE SHOWING RELATION OF WEIGHTS AND MEASURES OF THE METRIC SYSTEM TO APPROXIMATE WEIGHTS AND MEASURES OF THE U. S.

MEASURES OF LENGTH.

One Myriameter

One Kilometer

One Hectometer

One Decameter

One METER

One Decimeter

One Centimeter

One Millimeter

One Myriagram

One Kilogram

One Hectogram

One Decagram

One Gram

One Decigram

One Centigram

One Milligram

One Myrialiter

One Kiloliter

One Hectoliter

One Decaliter

One Liter

One Deciliter

One Centiliter

One Milliliter

$=$ 10,000 meters
$=\quad$ 1,000 6
$=\quad$ 100 6
$=\quad$ 10 “6

$=\left\{\begin{array}{l}\text { the ten-millionth part of a } \\ \text { quarter of the Meridian of } \\ \text { the Earth }\end{array}\right\}=39.368$

$=32,800$ feet.

$=3,280$ - " "

$=328$ "

$=$ the tenth part of one meter $=3.936$

$=\left\{\begin{array}{l}\text { the one-hundredth part of } \\ \text { one meter }\end{array}\right\}=0.393\left(\frac{2}{5}\right)$ inch.

$=\left\{\begin{array}{l}\text { the one-thousandth part } \\ \text { of one meter }\end{array}\right\}=0.039\left(\frac{1}{25}\right)$ “

WEIGHTS.

$=10,000$ grams
$=1,000 \% 6$
$=\quad 100 \% 6$
$=\quad 106$

$=263 / 4$ pounds Troy.

$=22 / 3$ " "6

$=3 \frac{1}{4}$ ounces

$=2 \frac{1}{2}$ drams

inches.

66

$=\left\{\begin{array}{l}\text { the weight of a cubic cen- } \\ \text { timeter of water at } 4^{\circ} \mathrm{C} .\end{array}\right\}=15.434 \quad$ grains.

$=$ the tenth part of a gram $=\mathbf{I} .543(\mathrm{I} I / 2)$ "

$=\left\{\begin{array}{l}\text { the hundredth part of one } \\ \text { gram }\end{array}=0.154(1 / 6)\right.$ grain.

$=\left\{\begin{array}{l}\text { the thousandth part of one } \\ \text { gram }\end{array}\right\}=0.015\left(\frac{1}{64}\right)$ "

MEASURES OF CAPACITY.

$=\left\{\begin{array}{l}\text { Io cubic Meters or the } \\ \text { measures of Io Milliers of } \\ \text { water }\end{array}\right\}=2,600$ gallons.

$=\left\{\begin{array}{l}\text { I cubic Meteror the meas- } \\ \text { ure of I Miller of water }\end{array}\right\}=260$ "

$=\left\{\begin{array}{l}100 \text { cubic Decimeters or } \\ \text { the measure of I Quintal } \\ \text { of water }\end{array}\right\}=26$ "

$=\left\{\begin{array}{l}\text { Io cubic Decimeters or } \\ \text { the measure of I Myria- } \\ \text { gram of water }\end{array}\right\}=2.6$ "

$=\left\{\begin{array}{l}\text { I cubic Decimeter or the } \\ \text { measure of I Kilogram } \\ \text { of water }\end{array}\right\}=2.1$ pints.

$=\left\{\begin{array}{l}\text { IoO cubic Centimeters or } \\ \text { the measure of I Hecto- } \\ \text { gram of water }\end{array}\right\}=3.3$ ounces.

$=\left\{\begin{array}{l}\text { Io cubic Centimeters or } \\ \text { the measure of I Deca- } \\ \text { gram of water }\end{array}\right\}=\quad 2.7$ drams.

$=\left\{\begin{array}{l}\text { I cubic Centimeter or the } \\ \text { measure of I Gram of } \\ \text { water }\end{array}\right\}=$ I6.2 minims. 


\section{INDEX.}

A BDUCENS NERVE,.... I 87

Aberration, chromatic, . . . 235

, spheric, ........ 235

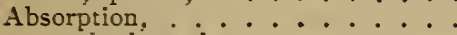

by lacteals,

by blood-vessels, . . .

- of oxygen in respiration,. . .

Accommodation of the eye, . . .

Adipose tissue, uses of, in the body, .

Adrenal bodies.

Adult circulation, establishment of, at

birth,

Air, atmospheric, composition of,

-, amount exchanged in respiration,

- changes in, during respiration,

Albumin, uses of, in the body,

Albuminoid substances,

Alcohol, action of,

Alimentary canal, development of,

— principles, classification of, . .

—, albuminous principles, ...

—, saccharine principles, . . .

- oleaginous principles, . . .

Allantois, development and function of

Amnion formation of, . . .

Animal heat, ..... . . . .

Anterior columns of spinal cord, . .

Area, germinal, . . . . . .

Areolar tissue,

Arteries, properties of, . . .

Articulations, . . . . . .

, classification of, . . . . . .

Asphyxia.

Astigmatism

Axis, cerebro-spinal, $\cdot \cdot \cdot \cdot \cdot$

Axis, cerebro-spinal, ............

cylinder of nerves, . . . . 73

BILE, ........ IIO

Bladder, urinary, . . . . I55 $_{55}$

Blastodermic membranes, .... 253

Blood, .......... Ir9

, composition of, plasma, . . I20

$\longrightarrow$, coagulation of, ....... I23

-, coloring matter of,

- , rapidity of flow in arteries, ...

_, rapidity of flow in capillaries,

$\longrightarrow$, corpuscles, ... .....

\section{I4I}

$4 I$

I54

262

I $4^{\circ}$

139

140
88

88

Con

I

I 2 I

I 25

I33

I33

I2I

I22
PAGE

Blood pressure, . . . . . . ${ }^{132}$

Bone, structure of, . . . . 44

Burdach, column of, . . . . . ${ }^{474}$

CANALS OF CUVIER, . . . 262

Capillary blood-vessels,. : . I I33

Capsule, internal,. . . . . . 204

C, external,........ 204

Cardiac cycle,. . . .

Cartilage,........ 43

Caudate nucleus, . . . . . 204

Cells, structure of, . . . . 33

- , manifestation of life by,. $\quad 35$

- of anterior horns of gray matter, I 73

Center for articulate language, . . . 2 I6

Cerebeilum, ........ 205 , forced movements of, . ... 206

Cerebral vesicles of embryo, . . . . 257

Cerebrum, ........ 207 , fissures and convolutions, . . 208

—, functions of,. . .... 2 II $_{3}$

- localization of functions, . . . $2 \mathrm{2I}_{4}$

- motor area of, . . . . . $\mathrm{II}_{4}$

Checial centers of, . . . 2 I5

Chemic composition of human body, . I5

Chorda dorsalis,...... . . 258

- tympani nerve, course and func-

tion of,.......... IgI

Chorion,........... 255

Chyle, .......... II7

Ciliary muscle, . . . . . 234

Circulation of blood,....... I25

Claustrum, ......... 204

Cochlea, ......... . ${ }^{2}+3$

Columns of spinal cord, . $\quad \mathrm{I}_{74}$

Connective tissues, physiologic properties of, . . . . . . 45

Corium, ...... $\quad$ I67

Corpora iWolffiana, ...... 260

Cquadrigemina, . . . . . 203

Corpus luteum, . . . . . 250

Cotriatum, ....... 204

Corti, organ of, . . . . . . 244

Cranial nerves, . . . . . . I83

Crura cerebri, ....... 202

Crystalline lens, ...... 230

DECIDUAL MEMBRANE, .. 255

Decussation of motor and sensory fibers, . . . . . I 75

Deglutition, ... ... 99 
Development of accessory structures of embryo. .

Digestion, ...

Ductuslarteriosus, . . . . .

工 venosus, .........

EAR

Embryo, development of, : .

Embryology, ............

Endolymph, .........

Epididymis, .....

Eustachian tube, ......

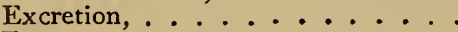

Eye, ... . . . . . . . , refracting apparatus of,

-, blind spot of, .......

FACIAL NERVE, . . . . - paralysis, symptoms of, . Fallopian tubes,

Fat, uses of, in the body, . . . .

Feces, . . . . . . .

Female organs of generation, ....

Fissures and convolutions of brain, .

Foods and dietetics,

—, animal,........

—, vegetable, ......

$\longrightarrow$, cereal,

- percentage, composition of, .

- daily amount required.

- , albuminous principles of, . .

$\longrightarrow$, saccharine principles of, . .

- oleaginous principles of, . .

--, inorganic principles of, . . .

Fovea centralis, ...... . 234

GALVANIC CURRENTS, Gangliect on nerves, . . . . .

Ganglia, ophilimic, . . . . . . , Gasserian,

, spheno-palatine, . . . .

_- otic,.. . . .

-

Gases of the intestine, . .

Gastric digestion,

- juice,

- - action of, . . . . . .

Generation, male organs of, . . .

- , female organs of, ......

Globules of the blood, . . . . . .

- of the lymph, .......

Glomeruli of the kidneys, . . . . .

Glosso-pharyngeal nerve,

Glottis, respiratory movements of, :

Glycogen,

Glycogenic function of the liver, .

Goll, column of, . . . . . .

Graafian follicles,........

HAIR, ........ 169

Hearing, sense of, . . . 237
PAGE

252

95

262

262

237

85
257

248

244

25I

239

I55

225

$23 \mathrm{I}$

$23^{6}$

I 89

I90

245

89

247

208

86

93

94
Heart, valves of, PAGE

I28

I29

I3I

I30

I:30

I3०

r26

I3I

I 21

230

235

I96

I53.

INCUS BONE, ....... 238

Insalivation, $\quad . . . . .996$

$\longrightarrow$, nervous circle of, . . . . 99

Inspiration, movements of thorax in, . $\quad$ I37

Internal capsule, . . . . . 204

- - , results of injury to, . . 204

- secretions, ....... I50

Intestinal juice, . . . . . . . 107

Iris, . . . . . . . . . 227

—, action of, ...... 235

KIDNEYS, ..... . . I55

I ABYRINTH OF INTERNAL

ear, ........ 243

—-, function of cochlea, . . . 244

- function of semicircular canals, 244

Language, articulate, center for, . . 2 216

Larynx, . . . . . 245

Lateral columns of spinal cord, . . . I74

Laws of muscular contraction, . . . 85

Lens, crystalline, ..... 230

Lime phosphate,. . . . . . 29

Liver, ....... ${ }^{2} \sigma_{3}$

- secretion of bile by, . . . . 165

- glycogenic function of, . . . 166

—, elaboration of blood, .... I65

\begin{tabular}{ll} 
Localization of functions in cerebrum, & 2I 4 \\
\hline
\end{tabular}

Lungs, .. . . . ${ }^{135}$

changes in blood while passing

through, ......... $\mathrm{I}_{42}$

Lymph, . . . . . . . II7

Lymphatic glands, . . . . II3

II3

MALleus BONE, . . . . $2{ }^{2} 8$

IV Mammary glands, . . . . ${ }^{4} 8$

Mastication,

96

$\begin{array}{rrr} & 95 \\ \text { Medulla oblongata, } & \ldots & .\end{array}$

, properties and functions of, . 199

Membrana tympani, . . . . 238

Menstruation, . . . . . . 240

Middle ear, $\ldots . . . \quad \ldots 228$ 
PAGE

Motor centers of cerebrum . * * . I49

Muscles, properties of, . . . . . . , changes in, during contraction,

- special physiology of, .... Muscle-fiber, histology of, . . . . 52

Myopia,.... ....... 235

NERVE, OLFACTORY, . . . I ${ }^{8} 3$

IN

, motor oculi . . . . . .

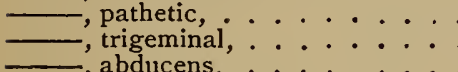

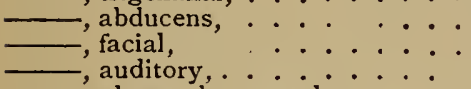

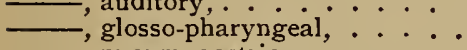

, pneumogastric, .

— spinal a ccessory, .....

_. hypoglossal, . . . . . . 196

- cells, structure of, . . . 70

- fibers, structure of . . . . . 72

- - terminations of, . . $\quad 7^{6}$

- force, rate of transmission of, .

posterior,

posterior, tissue, histology of, . . . .

Nerves, centrifugal and centripetal, .

Nerves, centrification of,

- relation of, to central nervous

system, development and nutrition of,
- , cranial, decussation of motor and sen-

sory, ... ........ I I7

, properties and functions of, .

-, spinal,

Nervous tissue, physiology of, $\cdot \dot{*}^{\circ} \cdot$.

, cerebro-spinal.

Neurone, sympathetic,

Nucleus caudatus,

- lenticularis

OLFACTORY NERVES

Ophthalmic ganglion

Optic nerves, , thalamus,

- functions of, ..., . . .

Organs of Corti, . . . . . . . . .

Otic ganglion, . . . . . . . .

Ovaries, . . . . . . . . .

Ovum,

, discharge of from the ovary,

Oxygen, absorption of, by hemoglobin

PANCREATIC JUICE,

Patheticus nerve,

Peptones

Perilymph, . • . • . . . .

Perspiration,

I05

243

170
Petrosal nerves, large and small.

PAGE

Phonation, . I9o

Physiology, definition of, . . . . . 247

Placenta, formation and function of, . $\quad 256$

Pleura, . . . . . . . 136

Pneumogastric nerve, . . . . . . . 192

Pons Varolii, . . . . . . . . . 2OI

Portal vein, ....... . . .

Posterior columns of spinal curd, . . $\quad 182$

, functions of, ...... 182

Prehension, . . . . . . . . . 95

Presbyopia, . . . . . . 235

Pressure of blood in arteries, . . . 132

Proximate principles, . . . . . 16

—, inorganic, . . . . . 29

-

$\longrightarrow$, organic, nitrogenized, . . . 23

$\longrightarrow$, quantity of chemic elements in

body,.............. I6

Ptyalin, . . . . . . . . . 98

Pulse, ...... . 132

Pyramidal tracts, ....... I 74

\section{RED CORPUSCLES OF}

$R$ blood, . . I2I

Reflex movements of spinal cord, .

Retion, laws of, ..... I77-I8I

Reproduction, ........ 248

Respiration, ........ 135

—, movements of, ...... 137

—, types of, ..... 139

Retin nervous circle of, ..... I38

Retina, ......... 229

Rigor mortis, . . ...... 54

SALIVA,

Sebaceous g. ....... 98

Secretion, . . . . r r

Semen, .............. 252

Semicircular canals, . . . . . 243

Sight, sense of, . . . . . 226

Skeleton, $\ldots \ldots \ldots . . . . .46$

skin..........

Smell, sense of, . . . . . . 225

Sounds of heart,. . . . . . . 129

Spermatozoa, ........ 252

Spheno-palatine ganglion, ..... 219

Spinal accessory nerve, : . . . . . 195

— cord ....... I I $_{7}$

205 - cord, membranes of, . . . . $\quad$ I $7 x$

$244 \longrightarrow$, structure of white matter, . . ${ }^{2} 73$

219 —, structure of gray matter, . . I I3

$248 \longrightarrow$ properties of, . . . . . I74

248 , function of, as a conductor, .. I8I

-, as an independent center, . I 76

- decussation of motor and sen-

sory fibers,

I 75
I 78

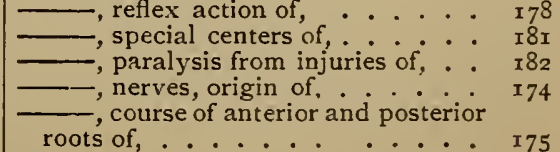

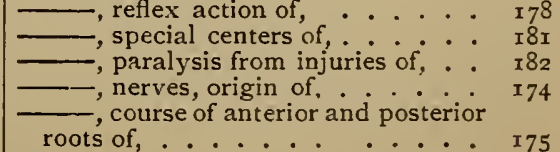

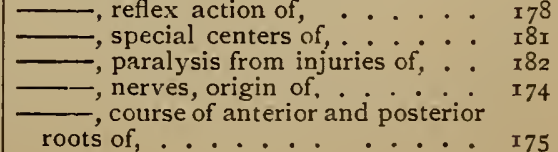


PAGE

Starvation, phenomena of, $\therefore \ldots{ }_{86}$

Stomach .. ....... I00

Submaxillary ganglion . . . . . 219

Sudoriparous glands, . ..... I70

Sugar, uses of, in the body, $\ldots \ldots 89$

Supra-renal capsules, . . . . . . I ${ }^{54}$

Sympathetic nervous system, . . . 218

TASTE, SENSE OF, . . . 223

T

Teeth, . . . . . . . . 95

Tensor tympani muscle, . . . . 24I

Testicles, .. . . . . 25I

Thoracic duct, . . . . . . II5

Thorax, enlargement of, in inspiration, 137

Thyroid gland, . . . . . . I5I

Tissues, physiology of, . . . . . 39

Tongue, .......... 223

—, motor nerve of, . . . . . . 224

Tonsory nerve of, . . . . . 224

Touch, sense of, . . . . . . 292

Türck, column of, . . . . . . . . ${ }^{\text {I74 }}$

MBILICAL CORD,

$\ldots \ldots 254$

Uric acid, $\quad \ldots \ldots \ldots \ldots{ }^{167}$

Urination, nervous mechanism of, . . ${ }_{159}$

Urine, . . . . . I59

$\longrightarrow$, composition of, . . . . I60

ents secreted daily, . . . .... 160

Uterus, . . . . . . . . . . 249

VAPOR, WATERY, OF

breath, ........ 150

Vascular glands, . . . . . I50

Vaso-motor nerves, origin of, . . . 200

Veins,. ........ I33

Vertebral column, . . . . . . . 49

Vesiculæ seminales, . . . . . . . 251

Vision, psychic center for, . . . 218

Vital capacity of lungs, . . . . . I40

Vocal cords, . . . . . . . 246

Voice, ........... 245

W ATER, AMOUNT OF, IN

Wolffian bodies, $\ldots \ldots \ldots \ldots{ }^{26}$ 



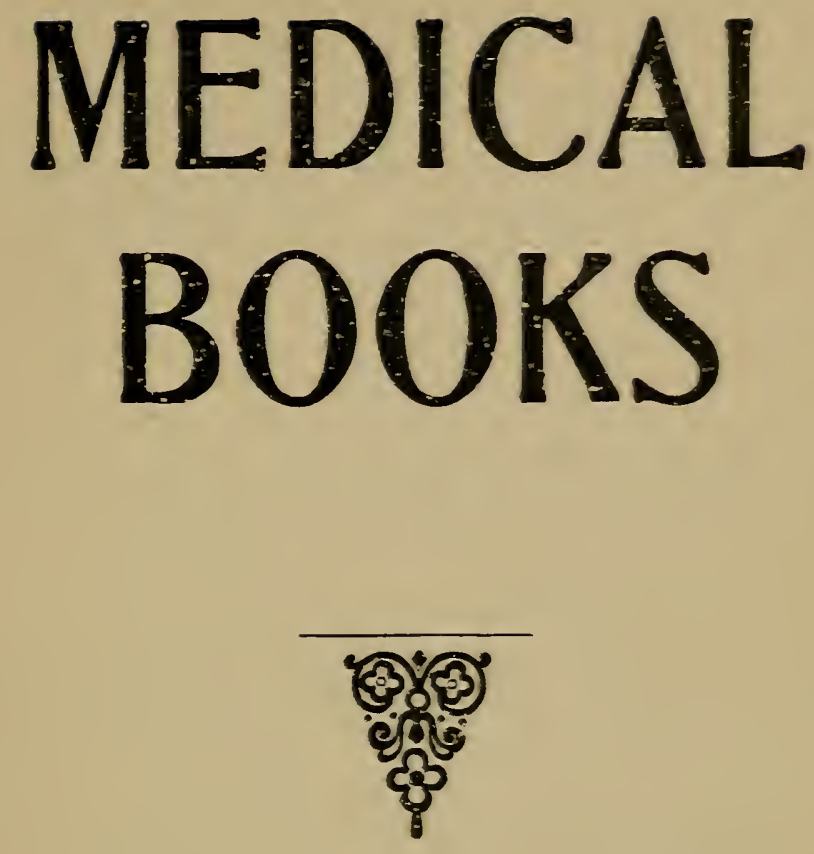

There have been sold more than 140,000 copies of Gould's Dictionaries See Page 12

P. BLAKISTON'S SON \& COMPANY PUBLISHERS OF MEDICAL AND SCIENTIFIC BOOKJ 1012 WALNUT STREET, PHILADELPHIA 


\section{Montgomery's Gynecology}

A PRACTICAL TEXT-BOOK

A modern comprehensive Text-Book. By EDWARD E. Montgomery, M.D., Professor of Gynecology in Jefferson Medical College, Philadelphia; Gynecologist to the Jefferson and St. Joseph's Hospitals, etc. 527 Illustrations, many of which are from original sources. 800 pages. Octavo.

Cloth, $\$ 5.00$; Leather, $\$ 6.00$

* * This is a systematic modern treatise on Diseases of Women. The author's aim has been to produce a book that will be thorough and practical in every particular. The illustrations, nearly all of which are from original sources, have for the most part been drawn by special artists who, for a number of months, devoted their sole attention to this work.

"The book is one that can be recommended to the student, to the general practitioner-who must sometimes be a gynecologist to a certain extent whether he will or not-and to the specialist, as an ideal and in every way complete work on the gynecology of to-day-a practical work for practical workers." - The Journal of the American Medical Association.

\section{Byford's Gynecology}

Third Revised Edition

\section{A MANUAL FOR STUDENTS AND PHYSICIANS}

By Henry T. Byford, M.D., Professor of Gynecology and Clinical Gynecology in the College of Physicians and Surgeons of Chicago; Professor of Clinical Gynecology, Women's Medical School of Northwestern University, and in Post-Graduate Medical School, etc. Third Edition, Enlarged. $\quad 363$ Illustrations, many of which are from original drawings and several of which are Colored. $12 \mathrm{mo}$.

$$
\text { Cloth, } \$ 3.00
$$

"As a book to help the student to quickly review what ought to be gotten up, so as to be prepared for the early examination, it is of great service. Such a book would also make a most excellent textbook for the college class room."-Virginia Medical Semi-Monthly, Richmond. 


\section{By JAMES TYSON, M.D.,}

Professor of Medicine, University of Pennsylvania, Physician to the Philadelphia Hospital, etc.

\section{The Practice of Medicine. Second Edition.}

A Text-Book for Physicians and Students, with Special Reference to Diagnosis and Treatment. With Colored Plates and many other Illustrations. Second Edition, Revised and Enlarged. 127 Illustrations. 8vo. 1222 pages.

Cloth, $\$ 5.50$; Leather, $\$ 6.50$; Half Russia, $\$ 7.50$

*** This edition has been entirely reset from new type. The author has revised it carefully and thoroughly, and added much new material and 37 new illustrations.

"We are firmly convinced that at the present time Dr. Tyson's hook on Practice can be most heartily commended to both the practitioner and student as a safe, reliable, and thuroughly up-to-date guide in the practice of medicine."-The Therapeutic Gazette.

"The clinical descriptions are clear and full, and the methods of treatment described are those generally recognized as being the most modern and satisfactory."-The London Lancet.

\section{Guide to the Examination of Urine. Tenth Edition.}

For the Use of Physicians and Students. With Colored Plate and Numerous Illustrations Engraved on Wood. Tenth Edition, Revised, Enlarged, and in many parts entirely rewritten.

Cloth, \$I.5O

** A French translation of this book has been published in Paris.

"The book is probably more widely and generally known and appreciated than any of its similars in subject and scope." -New York Medical Journal.

"The book is a reliable one, and should find a place in the library of every practitioner and student of medicine." - Buston Medical and Surgical Journal.

\section{Handbcok of Physical Diagnosis. Fourth Edition.}

Revised and Enlarged. With two Colored Plates and 55 other Illustrations. 298 pages. I 2 mo. Cloth, $\$ \mathbf{I} .50$

"Like everything else emanating from this distinguished author this little book is replete with practical information from beginning to end." - The Chicago Medical Recorder.

"The author approaches his subject from a practical point of view and the little work will prove a good friend to the student." The American Journal of the Medical Sciences. 


\section{MORRIS' ANATOMY}

Rewritten-Revised-Improved

\section{WITH MANY NEW ILLUSTRATIONS}

Out of 102 of the leading medical schools 60 recommend "Morris." It contains many features of special advantage to students. It is modern, up-to-date in every respect. It has been carefully revised, the articles on Osteology and Nervous System having been rewritten. Each copy contains the colored illustrations and a Thumb Index.

Octavo. With 838 Illustrations, of which a large number are printed in colors

\section{CLOTH, \$6.00; LEATHER, \$7.00}

"The ever-growing popularity of the book with teachers and students is an index of its value, and it may safely be recommended to all interested."-From The Medical Record, New York.

"Of all the text-books of moderate size on human anatomy in the English language, Morris is undoubtedly the most up to-date and accurate."-From The Philadelphia Medical Journal.

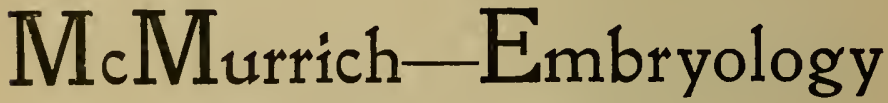

Nearly Ready. 276 Illustrations

A Text-Book for Medical Students. By J. PLAYFaIR

McMurrich, Professor of Anatomy, Medical Department, University of Michigan. 


\section{POTTER'S MATERIA IMDICA, PHARMACY, AND THERAPEUTICS}

\section{An Exhaustive Handbook}

Including the Action of Medicines, Special Therapeutics of Disease, Official and Practical Pharmacy, and Minute Directions for Prescription Writing, etc. Including over $65^{\circ}$ Prescriptions and Formulæ. By Samuel O. L. Potter, M.A., M.D., M.R.C.P. (Lond.), formerly Professor of the Principles and Practice of Medicine, 'Cooper Medical College, San Francisco; Major and Brigade Surgeon, U. S. Vol. Ninth Edition, Revised and Enlarged. 8vo.

With Thumb Index in each copy.

Cloth, $\$ 5.00$; Leather, $\$ 6.00$

* * This is the most complete and trustworthy book for the use of students and physicians. Students who purchase it will find it to contain a vast deal of information not in the usual text-books arranged in the most practical manner for facilitating study and reference. It cannot be surpassed as a physician's working book.

WHITE AND WILCOX. Materia Medica, Pharmacy, Pharmacology, and Therapeutics. Fifth Edition.

A Handbook for Students. By W. HALE White, M.D., F.R.C.P., etc., Physician to, and Lecturer on Materia Medica and Therapeutics at, Guy's IHospital, etc. Fifth American Edition, Revised by Reynold W. Wilcox, M A , M.D., LL.D., Professor of Clinical Medicine and Therapeutics at the New York Post-Graduate Medical School and Hospital ; Visiting Physician, St. Mark's Hospital ; Assistant Visiting Physician, Bellevie Hospital. I 2 mo.

Cloth, $\$ 3.00$; Leather, $\$ 3.50$ 
SUBJECT [INDEX.

\section{Gould's Medical Dictionaries, $=\quad=$ Page 12 Morris' Anatomy, New Edition, = Page 4 Compends for Students, $=\quad=\quad=$ Page 26}

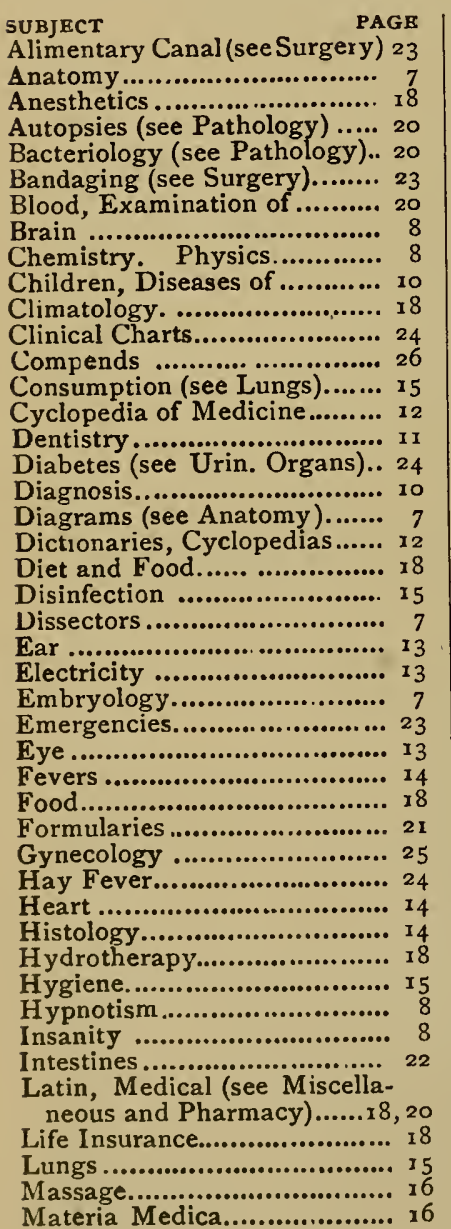

SUBJECT. PAGB Mechanotherapy.................. 16 Medical Jurisprudence ........... 17 Mental Therapeutics............. 8 Microscopy ...................... 17 Milk Analysis(see Chemistry) 8 Miscellaneous ...................... 18 Nervous Diseases ................. 18

Nose.................................. 24

Nursing ............................. I9

Obstetrics............................. 20

Ophthalmology..................... I I $_{3}$

Organotherapy .................... I8

Osteology (see Anatomy)....... 7

Pathology ............................. 20

Pharmacy ............................ 20

Physical Diagnosis ............... II

Physical Training................. 16

Physiology ........................ 2 I

Pneumotherapy................... I 8

Poisons (see Toxicology) ........ 17

Practice of Medicine............ 22

Prescription Books (Pharm'y), 2 I

Refraction (see Eye) ............ I3

Rest .................................. 18

Sanitary Science ................... I 5

Skin.................................. 23

Spectacles (see Eye).............. 13 Spine (see Nervous Diseases) 18 Stomach............................ 22

Students' Compends ............. 26

Surgery and Surg'l Diseases, 23

Technological Books............ 8

Temperature Charts............... 24

Therapeutics ....................... 16

Throat ............................... 24

Toxicology ......................... 17

Tumors (see Surgery)........... 23

U. S. Pharmacopœia ............. 2 I

Urinary Organs .................. 24

Urine .................................. 24

Venereal Diseases................. 25

Veterinary Medicine............. 25

Visiting Lists, Physicians'.

(Send for Special Circular.)

Water Analysis.................... I 5

Women, Diseases of............. 25

Self-Examination for Medical Students. 3500 Questions on Medical Subjects, with References to Standard Works in which the correct replies will be found. Together with Questions from State Examining Boards. 3 d Edition. Paper Cover, ro cts. 
SPECIAL NOTE. - The prices given in this catalogue are net, no discount can be allowed retail purchasers under any consideration. This rule has been established in order that everyone will be treated alike, a general reduction in former prices having been made to meet previous retail discounts. Upon receipt of the advertised price any book will be forwarded by mail or express, all charges prepaid.

\section{ANATOMY. EMBRYOLOGY.}

MORRIS. Text-Book of Anatomy. Revised and Enlarged Edition. 838 Illustrations, 269 of which are printed in coiors. Thumb Index in Each Copy. $\quad$ Cloth, $\$ 6.0$; Leather, $\$ 7.00$ "The ever-growing popularity of the book with teachers and students is an index of its value."-Medical Record, Nerw York.

BROOMELL. Anatomy and Histology of the Human Mouth and Teeth. 2d Edition, Enlarged. 337 Illustrations. $\$ 450$

CAMPBELL. Dissection Outlines. Based on Morris' Anatoiny. 2d Edition.

DEAVER. Surgical Anatomy. A Treatise on Anatomy in its Application to Medicine and Surgery. With 450 very Handsome fullpage Illustrations Engraved from Original Drawings made by special Artists from dissections prepared for the purpose. Three Volumes.

Cloth, \$21.00; Half Morocco or Sheep, \$24.00; Half Russia, \$27.00

GORDINIER. Anatomy of the Central Nervous System. With 271 Illustrations, many of which are original. Cloth, $\$ 6.00$

HEATH. Practical Anatomy. 8th Edition. 300 Illus. \$4.25

HOLDEN. Anatomy. A Manual of Dissections. Revised by A. Hewson, M.D., Demonstrator of Anatomy, Jefferson Medical College, Philadelphia. 320 handsome Illustrations. 7 th Edition. In two compact I2mo Volumes. 850 Pages. Large New Type.

Vol. I. Scalp-Face-Orbit-Neck-Throat-Thorax-Upper Extremity. $\$ 1.50$

Vol. II. Abdomen-Perineum-Lower Extremity-Brain-EyeEar-Mammary Gland-Scrotum-Testes. \$I.50

HOLDEN. Human Osteology. Comprising a Description of the Bones, with Colored Delineations of the Attachments of the Muscles. The General and Microscopical Structure of Bone and its Development. With Lithographic Plates and numerous Illus. 8th Ed. $\$ 5.25$

HOLDEN. Landmarks. Medical and Surgical. ${ }^{\text {th }}$ Ed. .75 HUGHES AND KEITH. Dissections. With 527 Colored Plates and other Illustrations. In three Parts. Just Ready.

I, Upper and Lower Extremity.

II, Abdomen-Thorax.

$\$ 3.00$

III, Head-Neck-Central Nervous System.

$\$ 3.00$

MACAIISTER. Humatic and Topograph ical. 8 I6 Illustrations.

Cloth, $\$ 5.00$; Leather, $\$ 6.00$

McMURRICH. Embryology. 270 Illustrations. Nearly Ready.

MARSHALL. Physiological Diagrams. Eleven Life-Size Colored Diagrams (each seven feet by three feet seven inches). Designed for Demonstration before the Class.

In Sheets, Unmounted, $\$ 40.00$; Backed with Muslin and Mounted on Rollers, $\$ 60.00$; Ditto, Spring Rollers, in Handsome Walnut Wall Map Case, \$100.00; Single Plates-Sheets, \$5.00; Mounted, \$7.50 Explanatory Key, .50. Purchaser must pay freight charges.

MINOT. Embryology. Illustrated.

In Press.

POTTER. Compend of Anatomy, Including Visceral Anatomy. 6 th Ed. 16 Lith. Plates and 117 other Illus. .80 ; Interleaved, $\$ 1.00$

WILSON. Anatomy. IIth Edition. 429 Illus., 26 Plates. $\$ 5.00$ 


\section{BRAIN AND INSANITY (see also Nervous Diseases).}

BLACKBURN. A Manual of Autopsies. Designed for the Use of Hospitals for the Insane and other Public Institutions. Ten fullpage Plates and other Illustrations.

$\$ 1.25$

CHASE. General Paresis. Illustrated. Just Ready. \$r 75

DERCUM. Mental Therapeutics, Rest, Suggestion. See Cohen, Physio.ogic Therapeutics, page 16.

GORDINIER. The Gross and Minute Anatomy of the Central Nervous System. With full-page and other Illustrations. $\$ 6.00$

HORSLEY. The Brain and Spinal Cord. The Structure and Functions of. Numerous Illustrations. $\$ 2.50$

IRELAND. The Mental Affections of Children. 2d Ed. \$4.00

LEWIS (BEVAN). Mental Diseases. A Text-Book Having Special Reference to the Pathological Aspects of Insanity. 26 Lithographic Plates and other Illustrations. 2d Ed. $\$ 7.00$

MANN. Manual of Psychological Medicine. $\$ 3 . \infty$

PERSHING. Diagnosis of Nervous and Mental Disease.

Illustrated $\$ 1.25$

REGIS. Mental Medicine. Authorized Translation by H. M. BANNISTER, M.D. $\$ 2 . \infty$

SCHOFIELD. The Force of Mind. Just Ready. $\$ 2 \infty$

STEARNS. Mental Diseases. With a Digest of Laws Relating to Care of Insane. Illustrated. Cloth, $\$ 2.75$; Sheep, \$3.25

TUKE. Dictionary of Psychological Medicine. Giving the Definition, Etymology, and Symptoms of the Terms used in Medical Psychology, with the Symptoms, Pathology, and Treatment of the Recognized Forms of Mental Disorders. Two volumes. \$10.00 WOOD, H. C. Brain and Overwork.

\section{CHEMISTRY AND TECHNOLOGY.}

Special Catalogue of Chemical Books sent free upon application.

ALLEN. Commercial Organic Analysis. A Treatise on the Modes of Assaying the Various Organic Chemicals and Products Employed in the Arts, Manufactures, Medicine, etc., with concise methods for the Detection of Impurities, Adulterations, etc. 8vo.

Vol. I. Alcohols, Neutral Alcoholic Derivatives, etc., Ethers, Vegetable Acids, Starch, Sugars, etc. 3d Edition. $\$ 4.50$

Vol. II, Part I. Fixed Oils and Fats, Glycerol, Explosives, etc. 3 d Edition. $\$ 3.50$

Vol. II, Part II. Hydrocarbons, Mineral Oils, Lubricants, Benzenes, Naphthalenes and Derivatives, Creosote, Phenols, etc. $3 \mathrm{~d}$ Ed. $\$ 3.50$

Vol. II, Part III. Terpenes, Essential Oils, Resins, Camphors, etc. 3d Edition.

Preparing.

Vol. III, Part I. Tannins, Dyes and Coloring Matters. 3d Edition. Enlarged and Rewritten. Illustrated. $\$ 4.50$

Vol. III, Part II. The Amines, Hydrazines and Derivatives, Pyridine Bases. The Antipyretics, etc. Vegetable Alkaloids, Tea, Coffee, Cocoa, etc. 8vo. 2d Edition. 4.50

Vol. III, Part III. Vegetable Alkaloids, Non-Basic Vegetable Bitter Principles. Animal Bases, Animal Acids, Cyanogen Compounds, etc. 2d Edition, 8vo. $\$ 4.50$

Vol. IV. The Proteids and Albuminous Principles. 2d Ed. \$4.50 BAILEY AND CADY. Qualitative Chemical Analysis. \$r.25 
BARTLEY. Medical and Pharmaceutical Chemistry. A Text-Book for Medical, Dental, and Pharmaceutical Students. With Illustrations, Glossary, and Complete Index. 5th Edition. \$3.00

BARTLEY. Clinical Chemistry. The Examination of Feces, Saliva, Gastric Juice, Milk, and Urine. $\$$ \$.0o

BLOXAM. Chemistry, Inorganic and Organic. With Experiments. 9th Ed., Revised. 28r Engravings. Preparing.

BUNGE. Physiologic and Pathologic Chemistry. From the Fourth German Enlarged Edition. Just Ready. \$3.co

CALDWELL. Elements of Qualitative and Quantitative Chemical Analysis. 3d Edition, Revised. \$1.00

CAMERON. Oils and Varnishes. With Illustrations. $\$ \mathbf{\$ 2 . 2 5}$

CAMERON. Soap and Candles, 54 Illustrations. $\$ 2.00$

CLOWES AND COLEMAN. Quantitative Analysis, 5th Edition. 122 Illustrations. $\$ 3.50$

COBLENTZ. Volumetric Analysis. Illustrated. \$1.25

CONGDON. Laboratory Instructions in Chemistry. With Numerous Tables and 56 lllustrations. \$1.00

GARDNER. The Brewer, Distiller, and Wine Manufacturer. Illustrated. $\$$ \$1.50

GRAY. Physics. Volume I. Dynamics and Properties of Matter. 350 Illustrations. $\$ 4.50$

GROVES AND THORP. Chemical Technology. The Application of Chemistry to the Arts and Manufactures.

Vol. I. Fuel and Its Applications. 607 Illustrations and 4 Plates.

Cloth, $\$ 5.00 ; 1 / 2$ Mor., $\$ 6.50$

Vol. II. Lighting. Illustrated. Cloth, $\$ 4.00 ; 1 / 2$ Mor., $\$ 5.50$

Vol. III. Gas Lighting. $\quad$ Cloth, \$3.50; $1 / 2$ Mor., \$4.50

Vol. IV. Electric Lighting. Photometry. In Press.

HEUSLER. The Terpenes. Just Ready. $\$ 4.00$

HOLLAND. The Urine, the Gastric Contents, the Common Poisons, and the Milk. Memoranda, Chemical and Microscopical, for Laboratory Use. 6th Ed. Illustrated and interleaved, \$1.00

LEFFMANN. Compend of Medical Chemistry, Inorganic and Organic. 4th Edition, Revised. .80 ; Interleaved, \$1.00

LEFFMANN. Analysis of Milk and Milk Products. 2d Edition, Enlarged. Illustrated.

LEFFMANN, Water Analysis. For Sanitary and Technic Purposes. Illustrated. 4th Edition.

LEFFMANN. Structural Formulæ. Including 180 Structural and Stereo-Chemical Formulæ. 12mo. Interleaved. \$1.00

LEFFMANN AND BEAM. Select Methods in Food Analysis. Illustrated. $\$ 2.50$

MUTER. Practical and Analytical Chemistry. 2d American from the Eighth English Edition. Revised to meet the requirements of American Students. 56 Illustrations. $\$$ 1.25

OETTEL. Exercises in Electro-Chemistry. Illustrated. $\quad .75$

OETTEL. Electro-Chemical Experiments. Illustrated. $\quad .75$

RICHTER. Inorganic Chemistry. 5th American from roth German Edition. Authorized translation by EDGAR F. SM1Th, M.A., PH.D. 89 Illustrations and a Colored Plate. $\$$ \$1.75

RICHTER. Organic Chemistry. 3d American Edition. Trans. from the 8th German by EDGAR F. SMrTH. Illustrated. 2 Volumes. Vol. I. Aliphatic Series. 625 Pages. $\$ 3.00$ Vol. II. Carbocyclic Series. 671 Pages. $\$ 3.00$ 
ROCKWOOD. Chemical Analysis for Students of Medicine, Dentistry, and Pharmacy. Illustrated. $\$ \mathrm{r} .50$ SMITH. Electro-Chemical Analysis. 2d Ed. 28 Illus. \$1.25 SMITH AND KELLER. Experiments. Arranged for Students in General Chemistry. 4th Edition. Illustrated .60

SUTTON. Volumetric Analysis. A Systematic Handbook for the Quantitative Estimation of Chemical Substances by Measure, Applied to Liquids, Solids, and Gases. 8th Edition, Revised. 112 Illustrations.

$\$ 5.00$

SYMONDS. Manual of Chemistry. 2d Edition. \$2.00

TRAUBE. Physico-Chemical Methods. Translated by Hardin. 97 Illustrations. $\$ \mathbf{1 1 . 5 0}$

THRESH. Water and Water Supplies. 3d Edition. \$2.00 ULZER AND FRAENKEL. Chemical Technical Analysis. Translated by Fleck. Illustrated. \$1.25

WOODY. Essentials of Chemistry and Urinalysis. 4th Edition. Illustrated. \$I.50 ** Special Catalogue of Books on Chemistry free upon application.

\section{CHILDREN.}

HATFIELD. Compend of Diseases of Children. With a Colored Plate. 3d Edition. In Press. .80; Interleaved, \$1.00 IRELAND. The Mental Affections of Children. Idiocy, Imbecility, Insanity, etc. 2d Edition. $\$ 4.00$

POWER. Surgical Diseases of Children and their Treatment by Modern Methods. Illustrated. $\$ 2.50$

STARR. The Digestive Organs in Childhood. The Diseases of the Digestive Urgans in Infancy and Childhood. 3d Edition, Rewritten and Enlarged. Illustrated.

$\$ 3.00$

STARR. Hygiene of the Nursery. Including the General Regimen and Feeding of Infants and Children, and the Domestic Management of the Ordinary Emergencies of Early Life, Massage, etc. 6th Edition. 25 Illustrations. $\$ 1.00$

SMITH. Wasting Diseases of Children. 6th Edition. \$2.00

TAYLOR AND WELLS. The Diseases of Children. 2d Edition, Revised and Enlarged. Illustrated. 8vo. \$4.50

"It is well worthy the careful study of both student and practitioner, and can not fail to prove of great value to both. We do not hesitate to recommend it."-Boston Medical and Surgical Journal.

\section{DIAGNOSIS.}

BROWN. Medical Diagnosis. A Manual of Clinical Methods.

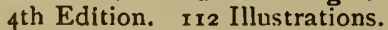

Cloth, \$2.25

DA Costa. Clinical Hematology. A Practical Guide to Examination of Blood. 6 Colored Plates. 48 other Illustrations. Just Ready.

Cloth, $\$ 5.00$; Sheep, $\$ 6.00$

EMERY. Bacteriological Diagnosis. 2 Colored Plates and 32 other Illustrations. Just Ready.

$\$ \pi .50$

MEMMINGER. Diagnosis by the Urine. 2 d Ed. 24 Illus. \$1.00 
PERSHING. Diagnosis of Nervous and Mental Diseases. Illustrated. $\$ 1.25$

STEELL. Physical Signs of Pulmonary Disease. \$1.25

TYSON. Hand-Book of Physical Diagnosis. For Students and Physicians. By the Professor of Clinical Medicine in the University of Pennsylvania. Illus. 4th Ed., Improved and Enlarged. With Two Colored and 55 other Illustrations. \$1.50

\section{DENTISTRY.}

Special Catalogue of Dental Books sent free upon application.

BARRETT. Dental Surgery for General Practitioners and Students of Medicine and Dentistry. Extraction of Teeth. etc. 3d Edition. Illustrated.

$\$ 1.00$

BROOMELL. Anatomy and Histology of the Human Mouth and Teeth. Second Edition, Revised and Enlarged. $337 \mathrm{Hand}$ some Illustrations.

$\$ 4.50$

FILLEBROWN. A Text-Book of Operative Dentistry. Written by invitation of the National Association of Dental Faculties. Illustrated.

$\$ 2.25$

QORGAS. Dental Medicine. A Manual of Materia Medica and Therapeutics. 7 th Edition. Cloth, $\$ 4.00$; Sheep, $\$ 5.00$

GORGAS. Questions and Answers for the Dental Student. Embracing all the subjects in the Curriculum of the Dental Student. Octavo.

$\$ 6.00$

HARRIS. Principles and Practice of Dentistry. Including Anatomy, Physiology, Pathology, Therapeutics, Dental Surgery, and Mechanism. I $3^{\text {th }}$ Edition. Revised by F. J. S. GoRGAS, M.D., D.D.S. 1250 Illustrations. Cloth, $\$ 6.00$; Leather, \$7.00

HARRIS. Dictionary of Dentistry. Including Definitions of Such Words and Phrases of the Collateral Sciences as Pertain to the Art and Practice of Dentistry. 6th Edition. Revised and Enlarged by FrRDinand F. S. Gorgas, M.D., D.D.S. Cloth, $\$ 5.00$; Leather, $\$ 6.00$

RICHARDSON. Mechanical Dentistry, 7th Edition. Thoroughly Revised and Enlarged by DR. GRo. W. WARREN. 69I Illustrations.

Cloth, $\$ 5.00$; Leather, $\$ 6.00$

SMITH. Dental Metallurgy. 2d Edition. Illustrated. In Press.

TAFT. Index of Dental Periodical Literature. $\$ 2.00$

TOMES. Dental Anatomy. Human and Comparative. 263 Illustrations. 5th Edition. $\$ 4.0$

TOMES. Dental Surgery. 4th Edition. 289 Illustrations. $\$ 4.00$

WARREN. Compend of Dental Pathology and Dental Medicine. With a Chapter on Emergencies. 3d Edition. Illustrated.

.80 ; Interleaved, $\$ 1.25$

WARREN. Dental Prosthesis and Metallurgy. I2g Ills, \$1.25

WHITE. The Mouth and Teeth. Illustrated. 


\section{DICTIONARIES AND CYCLOPEDIAS}

GOULD. The Illustrated Dictionary of Medicine, Biology and Allied Sciences. Being an Exhaustive Lexicon of Medicine and those Sciences Collateral to it: Biology (Zoology and Botany), Chemistry, Dentistry, Parmacology, Microscopy, etc., with many useful Tables and numerous fine Iltustrations. I633 pages. 5th Ed. Sheep or Half Morocco, $\$ 10.00$; with Thumb Index, $\$ 11.00$ Half Russia, Thumb Index, \$ $2 . c 0$

GOULD. The Medical Student's Dictionary. Irth Edition. Illustrated. Including all the Words and Phrases Generally Used in Medicine, with their Proper Pronunciation and Definition, Based on Recent Medical Literature. With Table of Eponymic Terms and Tests and Tables of the Bacilli, Micrococci, Mineral Springs, etc., of the Arteries, Muscles, Nerves, Ganglia, Plexuses, etc. Irth Edition. Enlarged and illustrated with a large number of Engravings. 840 pages.

Half Morocco, $\$ 2.50$; with Thumb Index, $\$ 300$

GOULD. The Pocket Pronouncing Medical Lexicon. $4^{\text {th }}$ Edition. (30,000 Medical Words Pronounced and Defined.) Containing all the Words, their Definition and Pronunciation, that the Medical, Dental, or Pharmaceutical Student Generally Comes in Contact With; also Elaborate Tables of Eponymic Terms. Arteries, Muscles, Nerves, Bacilli, etc., etc., a Dose List in both English and Metric Systems, etc., Arranged in a Most Convenient Form for Reference and Memorizing. Fourth Edition, Revised and Enlarged. 838 pages. Full Limp Leather, Gilt Edges, $\$ 1.00$; Thumb Index, \$1.25 140,000 Copies of Gould's Dictionaries Have Been Sold.

GOULD AND PYLE. Cyclopedia of Practical Medicine and Surgery. Seventy-two Special Contributors. Illustrated. One Volume. A Concise Reference Handbook of Medicine, Surgery, Obstetrics, Materia Medica, Therapeutics, and the Various Specialties, with Particular Reference to Diagnosis and Treatment. Compiled under the Editorial Supervision of Georgr M. Gould, M.D., Author of "An Illustrated Dictionary of Medicine," etc.; and Waltre L. Pyle, M.D., Assistant Surgeon Wills Eye Hospital; formerly Editor "International Medical Magazine," etc., and Seventy-two Special Contributors. With many Illustrations. Large Square 8vo, to correspond with Gould's "Illustrated Dictionary." Full Sheep or Half Mor., \$10.00; with Thumb Index, $\$$ I 1.00

Half Russia, Thumb Index, $\$ 12.00$ net.

GOULD AND PYLE. Pocket Cyclopedia of Medicine and Surgery. Based upon above book and uniform in size with "Gould's Pocket Dictionary."

Full Limp Leather, Gilt Edges, \$1.00, with Thumb Index, \$1.25

HARRIS. Dictlonary of Dentistry. Including Definitions of Such Words and Phrases of the Collateral Sciences as Pertain to the Art and Practice of Dentistry. 6th Edition. Revised and Enlarged by Ferdinand J. S. GorGaS, M.D., D.D.S. Cloth, $\$ 5.00$; Leather, $\$ 6.00$

LONGLEY. Pocket Medical Dictionary. Cloth, .75

MAXWELL. Terminologia Medica Polyglotta. By Dr. Throdore Maxwrll, Assisted by Others.

The object of this work is to assist the medical men of any nationality In reading medical literature written in a language not their own. Each term is usually given in seven languages, viz.: English, French, German, Italian, Spanish, Russian, and Latin.

TREVES AND LANG. German-English Medical Dictionary. Half Calf, $\$ 3.25$ 


\section{EAR (see also Throat and Nose).}

BURNETT. Hearing and How to Keep It. Illustrated. .40

DALBY. Diseases and Injuries of the Ear. 4th Edition. 38 Wood Engravings and 8 Colored Plates.

$\$ 2.50$

HOVELL. Diseases of the Ear and Naso-Pharynx. Including Anatomy and Physiology of the Organ, together with the Treatment of the Affections of the Nose and Pharynx which Conduce to Aural Disease. I 28 Illustrations. 2d Edition.

$85.5^{\circ}$

PRITCHARD. Diseases of the Ear. 3d Edition, Enlarged. Many Illustrations and Formulæ.

$\$ 1.50$

\section{ELECTRICITY.}

BIGELOW, Plain Talks on Medical Electricity and Batteries. With a Therapeutic Index and a Glossary. 43 Illustrations. 2d Edition.

$\$ 1.00$

HEDLEY. Therapeutic Electricity and Practical Muscle Testing. 99 Illustrations. $\$ 2.50$

JACOBY. Electrotherapy. 2 Vols. Illustrated. See Cohen, Physiologic Therapeutacs, page 16.

JONES. Medical Electricity. 3d Edition. Ir7 Illus. \$3.00

\section{EYE.}

A Special Circular of Books on the Eye sent free upon application.

DONDERS. The Nature and Consequences of Anomalies of Refraction. With Portrait and Illustrations. Half Morocco, \$1.25

FICK. Diseases of the Eye and Ophthalmoscopy. Translated by A. B. HALE, M. D. 157 Illustrations, many of which are in colors, and a glossary.

Cloth, \$4.50; Sheep, \$5.50

GOULD AND PYLE. Compend of Diseases of the Eye and Refraction. Including Treatment and Operations, and a Section on Local Therapeutics. With Formula, Useful Tables, a Glossary, and III Illus., several of which are in colors. 2d Edition, Revised. Cloth, .80; Interleaved, $\$ 1.00$

GREEFF. The Microscopic Examination of the Eye. Illustrated.

HARLAN. Eyesight, and How to Care for It. 'Illus.

$\$ 1.25$

HARTRIDGE. Refraction. 104 Illustrations and Test Types. IIth Edition, Enlarged.

$\$ 1.50$

HARTRIDGE. On the Ophthalmoscope. 4th Edition. With 4 Colored Plates and 68 Wood-cuts.

$\$ 1.50$

HANSELL AND REBER. Muscular Anomalies of the Eye. Illustrated.

$\$ 1.50$

HANSELL AND BELL. Clinical Ophthalmology. Colored Plate of Normal Fundus and 120 Illustrations.
\$1.50

JENNINGS. Manual of Ophthalmoscopy. 95 Illustrations and I Colored Plate. 
MORTON. Refraction of the Eye. Its Diagnosis and the Correction of its Errors. 6th Edition.

OHLEMANN. Ocular Therapeutics. Authorized Translation, and Edited by Dr. Charles A. Oliver. \$r.75

PARSONS. Elementary Ophthalmic Optics. With Diagrammatic Illustrations. $\$ 2.00$

PHILLIPS. Spectacles and Eyeglasses. Their Prescription and Adjustment. 2d Edition. 49 Illustrations. $\$ 1 . \infty$

SWANZY. Diseases of the Eye and Their Treatment. 7 th Edition, Revised and Enlarged. I64 Illustrations, I Plain Plate, and a Zephyr Test Card.

$\$ 2.50$

\section{From The Ifedical News.}

"Swanzy has succeeded in producing the most intellectually conceived and thoroughly executed résume of the science within the limits he has assigned himself. As a 'students' handbook,' small in size and of moderate price, it can hardly be equaled."

THORINGTON. Retinoscopy, 4th Edition. Carefully Revised. Illustrated.

\section{$\$ 1.00$}

THORINGTON. Refraction and How to Refract. 200 Illustrations, I3 of which are Colored. 2d Edition. $\$$ x.50

WALKER. Students' Aid in Ophthalmology. Colored Plate and 40 other Illustrations and Glossary. 51.50

WRIGHT. Ophthalmology. 2d Edition, Revised and Enlarged. I 7 Illustrations and a Glossary.

$\$ 3.00$

\section{FEVERS.}

GOODALL AND WASHBOURN. Fevers and Their Treatment. Illustrated.

$\$ 3.00$

\section{HEART.}

THORNE. The Schott Methods of the Treatment of Chronic Heart Disease. Fourth Edition. Illustrated, Just Ready. 2.00

\section{HISTOLOGY.}

CUSHING. Compend of Histology. By H. H. Cushing, M.D., Demonstrator of Histology, Jefferson Medical College, Philadelphia. Illustrated. Nearly Ready.

.80 ; lnterleaved, $\$ 1.00$

STIRLING. Outlines of Practical Histology. 368 Illustrations. 2d Edition, Revised and Enlarged. With new Illustrations. \$2.00

STÖHR. Histology and Microscopical Anatomy. Edited by A. SCHAPER, M.D., University of Breslau, formerly Demonstrator of Histology, Harvard Medical School. Fourth American from 9 th German Edition, Revised and Enlarged. 379 Illustrations. $\$ 3.00$ 


\section{HYGIENE AND WATER ANALYSIS.}

Special Catalogue of Books on Hygiene sent free upon application.

CANFIELD. Hygiene of the Sick-Room. A Book for Nurses and Others. Being a Brief Consideration of Asepsis, Antisepsis, Disinfection, Bacteriology, Immunity, Heating, Ventilation, etc. \$1.25

CONN. Agricultural Bacteriology. Illustrated. \$2.50

COPLIN. Practical Hygiene. A Complete American Text-Book. I38 Illustrations. New Edition. Preparing.

HARTSHORNE. Our Homes. Illustrated.

.40

KENWOOD. Public Health Laboratory Work. I16 Illustrations and 3 Plates.

$\$ 2.00$

LEFFMANN. Select Methods in Food Analysis. 53 Illustrations and 4 Plates. $\$ 2.50$

LEFFMANN. Examination of Water for Sanitary and Technical Purposes. 4th Edition. Illustrated. \$1.25

LEFFMANN. Analysis of Milk and Milk Products. Illustrated. Second Edition. . \$1.25

LINCOLN. School and Industrlal Hygiene.

.40

McFARLAND. Prophylaxis and Personal Hygiene. Care of the Sick. See Cohen. Physiologic Therapeutics, page 16.

NOTTER. The Theory and Practice of Hygiene. $x_{5}$ Plates and $13^{8}$ other Illustrations. 8vo. 2d Edition. $\$ 7.00$

PARKES. Hygiene and Public Health. By Louis C. Parkes, M.D. 6th Edition. Enlarged. Illustrated. $\$ 3.00$

PARKES. Popular Hygiene. The Elements of Health. A Book for Lay Readers. Illustrated.

$\$ 1.25$

ROSENAU. Disinfection and Disinfectants. Illustrated. $\$ 2,00$

STARR. The Hygiene of the Nursery. Including the General Regimen and Feeding of Infants and Children, and the Domestic Management of the Ordinary Emergencies of Early Life, Massage, etc. 6th Edition. 25 Illustrations.

$\$ 1.00$

STEVENSON AND MURPHY. A Treatise on Hygiene. By Various Authors. In Three Octave Volumes. Illustrated.

Vol. I, $\$ 6 . \infty$; Vol. II, $\$ 6 . \infty$; Vol. III, $\$ 5 . \infty$

*** Each Volume sold separately. Special Circular upon application.

THRESH. Water and Water Supplies. 3d Edition. $\$ 2.00$

WILSON. Hand-Book of Hygiene and Sanitary Science. Wirh Illustrations. 8th Edition. $\$ 3.00$

WEYL. Sanitary Relations of the Coal-Tar Colors. Authorized Translation by HeNRY LEFrmanN, M.D., PH.D. \$I.25

\section{LUNGS AND PLEURA.}

KNOPF. Pulmonary Tuberculosis. Its Modern Prophylaxis and Treatment in Special Institutions and at Home. Illus. \$3.00

STEELL. Physical Signs of Pulmonary Disease. Illus. \$1.25 


\section{MASSAGE-PHYSICAL EXERCISE.}

OSTROM. Massage and the Original Swedish Movements. Their Application to Various Diseases of the Body. A Manual for Students, Nurses, and Physicians. Fifth Edition, Enlarged. 115 Illustrations, many of which are original. $\$ 1.00$

MITCHELL AND GULICK. Mechanotherapy, Physical Education, etc. Illustrated. See Cohen, Physiologic Therapeutics, below.

TREVES. Physical Education. Its Value, Methods, etc. .75

\section{MATERIA MEDICA AND THERA- PEUTICS.}

BIDDLE. Materia Medica and Therapeutics. Including Dose List, Dietary for the Sick, Table of Parasites, and Memoranda of New Remedies. 13th Edition, Revised. 64 Illustrations and a Clinical Index. $\quad$ Cloth, $\$ 4.00$; Sheep, $\$ 5.00$

BRACKEN. Outlines of Materia Medica and Pharmacology. \$2.75

COBLENTZ. The Newer Remedies. Including their Synonyms, Sources, Methods of Preparation, Tests, Solubilities, Doses, etc. 3d Edition, Enlarged and Revised.

$\$ 1.00$

COHEN. Physiologic Therapeutics. Methods other than DrugGiving useful in the Prevention of Disease and in the Treatment of the Sick. Mechanotherapy, Mental Therapeutics, Suggestion, Electrotherapy. Climatology, Hydrotherapy, Pneumatotherapy, Prophylaxis, Dietetics, Organotherapy, Phototherapy, Mineral Waters, Baths, etc. II Volumes, Octavo. Illustrated. (Subscription.)

Cloth, $\$ 27.50 ; 1 / 2$ mor., $\$ 38.50$

Special Descriptive Circular will be sent upon application.

DAVIS. Materia Medica and Prescription Writing. \$1.50

GORGAS. Dental Medicine. A Manual of Materia Medica and Therapeutics. 7th Edition, Revised. $\$ 4.00$

GROFF. Materia Medica for Nurses. with questions for Self Examination. 2d Edition, Revised and Improved.

In Press.

HELLER. Essentials of Materia Medica, Pharmacy, and Prescription Writing.

$\$ 1.50$

MAYS. Theine in the Treatment of Neuralgia. $1 / 2$ bound, .50

POTTER. Hand-Book of Materia Medica, Pharmacy, and Therapeutics, including the Action of Medicines, Special Therapeutics, Pharmacology, etc., including over 600 Prescriptions and Formulx. gth Edition, Revised and Enlarged. With Thumb Index in each copy. Just Ready.

Cloth, $\$ 5.00$; Sheep, $\$ 6.00$

POTTER. Compend of Materia Medica, Therapeutics, and Prescription Writing, with Special Reference to the Physiological Action of Drugs. 6th Edition.

.80 ; Interleaved, $\$ 1.00$

MURRAY. Rough Notes on Remedies. 4th Edition. \$1.25 
SAYRE. Organic Materia Medica and Pharmacognosy. An Introduction to the Study of the Vegetable Kingdom and the Vegetable and Animal Drugs. Comprising the Botanical and Physical Characteristics. Source, Constituents, and Pharmacopeial Preparations, Insects Injurious to Drugs, and Pharmacal Botany. With sections on Histology and Microtechnique, by W. C. Stevens. 374 Illustrations, many of which are original. $2 \mathrm{~d}$ Edition.

Cloth, $\$ 4.50$

TAVERA. Medicinal Plants of the Philippines. $\$ 2.00$

WHITE AND WILCOX. Materia Medica, Pharmacy, Pharmacology, and Therapeutics. 5th American Edition, Revised by ReYNold W. Wilcox, M.A., M.D., LL.D., Professor of Clinical Medicine and Therapeutics at the New York Post-Graduate Medical School.

Cloth, \$3.00; Leather, \$3.50

"The care with which Dr. Wilcox has performed his work is conspicuous on every page, and it is evident that no recent drug possessing any merit has escaped his eye. We believe, on the whole, this is the best book on Materia Medica and Therapeutics to place in the hands of students, and the practitioner will find it a most satisfactory work for daily use."-The Cleveland Medical Gazette.

\section{MEDICAL JURISPRUDENCE AND TOXICOLOGY.}

REESE. Medical Jurisprudence and Toxicology. A Text-Book for Medical and Legal Practitioners and Students. 5th Edition. Revised by Hznry LefrmanN, M.D. Clo., \$3.00; Leather, \$3.50

"To the student of medical jurisprudence and toxicology it is invaluable, as it is concise, clear, and thorough in every respect." - The American Journal of the Medical Sciences.

MANN. Forensic Medicine and Toxicology. Illus. \$6.50

TANNER. Memoranda of Poisons. Their Antidotes and Tests. 8th Edition, by Dr. HgnRy Lefrmann.

.75

\section{MICROSCOPY.}

CARPENTER. The Microscope and Its Revelations. 8th Edition, Revised and Enlarged. 817 Illustrations and 23 Plates. Cloth, $\$ 8.00$; Half Morocco, $\$ 9.00$

LEE. The Microtomist's Vade Mecum. A Hand-Book of Methods of Microscopical Anatomy. 887 Articles. 5th Edition, Enlarged.

$\$ 4.00$

OERTEL. Medical Microscopy. A Guide to Diagnosis, Elementary Laboratory Methods and Microscopic Technic. Izo Illustrations.

Nearly Ready.

REEVES. Medical Microscopy, including Chapters on Bacteriology, Neoplasms, Urinary Examination, etc. Numerous Illus. trations, some of which are printed in colors.

$\$ 2.50$

WETHERED. Medical Microscopy. A Guide to the Use of the Microscope in Practical Medicine. Io Illustrations. \$2.00 


\section{MISCELLANEOUS.}

BERRY. Diseases of Thyroid Gland. Illustrated. $\$ 4.00$ BURNETT. Foods and Dietaries. A Manual of Clinical Dietetics. 2d Edition.

BUXTON. Anesthetics. Illustrated. 3d Edition. \$r.50

COHEN. Organotherapy. See Cohen, Physiologic Therapeutics page 16.

DAVIS. Dietotherapy. Food in Health and Disease. With Tables of Dietaries, Relative Value of Foods, etc. See Cohen, Physiologic Therapeutics, page 16.

GOULD. Borderland Studies. Miscellaneous Addresses and Essays. 12mo.

$\$ 2.00$

GREENE. Medical Examination for Life Insurance. Illustrated. With Colored and other Engravings.

$\$ 4.00$

HAIG. Causation of Disease by Uric Acid. The Pathology of High Arterial Tension, Headache, Epilepsy, Gout, Rheumatism, Diabetes, Bright's Disease, etc. 5th Edition. $\$ 3.00$

HAIG. Diet and Food. Considered in Relation to Strength and Power of Endurance. 3d Edition.

HENRY. A Practical Treatise on Anemia. Half Cloth, .50

LEFFMANN. Food Analysis. Illustrated. \$2.50

NEW SYDENHAM SOCIETY'S PUBLICATIONS. Circulars upon application.

Per Annum, $\$ 8.00$

OSGOOD. The Winter and Its Dangers.

PACKARD. Sea Air and Sea Bathing.

.40

RICHARDSON. Long Life and How to Reach It.

ST. CLAIR. Medical Latin.

TISSIER. Pneumatotherapy. See Cohen, Physiologic Therapeutics, page 16.

TURNBULL. Artificial Anesthesia. 4th Edition. Illus. \$2.50 WEBER AND HINSDALE. Climatology and Health Resorts. Including Mineral Springs. 2 Vols. Illus'rated with Colored Maps. See Cohen, Physiologic Therapeutics, page 16.

WILSON. The Summer and Its Diseases.

.40

WINTERNITZ. Hydrotherapy, Thermotherapy, Phototherapy, Mineral Waters, Baths, etc. Illustrated. See Cohen, Physiologic Therapentics, page 16.

\section{NERVOUS DISEASES.}

DERCUM. Rest, Suggestion, Mental Therapeutics. See Cohen, Physiolos ic Therapeutics, page 16.

GORDINIER. The Gross and Minute Anatomy of the Central Nervous System. With $27 \mathrm{r}$ original Colored and other Illustrations.

Cloth, \$6.00; Sheep, \$7.00

GOWERS. Syphllis and the Nervous System. \$r.0 
GOWERS. Manual of Diseases of the Nervous System. A Complete Text-Book. Revised, Enlarged, and in many parts Rewritten. With many new Illustrations. Two volumes.

Vol. I. Diseases of the Nerves and Spinal Cord. 3d Edition, Enlarged. Cloth, $\$ 4.00$; Sheep, $\$ 5.00$ Vol. II. Diseases of the Brain and Cranial Nerves; General and Functional Disease. 2d Edition. Cloth, \$4.00; Sheep, $\$ 5.00$

GOWERS. Epilepsy and Other Chronic Convulsive Diseases. 2d Edition.

$\$ 3.00$

HORSLEY. The Brain and Spinal Cord. The Structure and Functions of. Numerous Illustrations. $\$ 2.50$

ORMEROD. Diseases of the Nervous System. 66 Wood Engravings.

$\$ 1.00$

PERSHING. Diagnosis of Nervous and Mental Diseases. Illustrated.

$\$ 1.25$

PRESTON. Hysteria and Certain Allied Conditions. Their Nature and Treatment. Illustrated. $\$ 2.00$

WOOD. Brain Work and Overwork.

.40

\section{NURSING (see also Massage).}

Special Catalogue of Books for Nurses sent free upon application.

CANFIELD. Hygiene of the Sick-Room. A Book for Nurses and Others. Being a Brief Consideration of Asepsis, Antisepsis, Disinfection, Bacteriology, Immunity, Heating and Ventilation, and Kindred Subjects for the Use of Nurses and Other Intelligent Women. \$1.25

CUFF. Lectures to Nurses on Medicine. Third Edition. \$1.25

DAVIS. Bandaging. Its Principles and Practice. I63 Original Illustrations. Just Ready.

$\$ 1.50$

DOMVILLE. Manual for Nurses and Others Engaged in Attending the Sick. 9th Edition. With Recipes for Sick-room Cookery, etc.

In Press.

FULLERTON. Obstetric Nursing. $4^{1}$ Ills. 5th Ed. \$1.0

FULLERTON. Surgical Nursing. $3 d \mathrm{dd}$. 69 Ills. $\$ 1.00$

GROFF. Materia Medica for Nurses. With Questions for Self-Examination. 2d Edition, Revised and Improved. Just Ready. \$1.25

HADLEY. General, Medical, and Surgical Nursing. A very Complete Manual, Including Sick-Room Cookery. Just Ready. \$1.25

HUMPHREY. A Manual for Nurses. Including General Anatomy and Physiology, Management of the Sick Room, etc. 23d Edition. 79 Illustrations.

$\$ 1 . \infty$

STARR. The Hygiene of the Nursery. Including the General Regimen and Feeding of Infants and Children, and the Domestic Management of the Ordinary Emergencies of Early Life, Massage, etc. 6th Edition. 25 Illustrations.

$\$ 1 . \infty$

TEMPERATURE AND CLINICAL CHARTS. See page 24.

VOSWINKEL. Surgical Nursing. Second Edition, Enlarged. II2 Illustrations. 


\section{OBSTETRICS.}

CAZEAUX AND TARNIER. Midwifery. With Appendix by Mundk. The Theory and Practice of Obstetrics, including the Diseases of Pregnancy and Parturition, Obstetrical Operations, etc. 8th Edition. Illustrated by Colored and other full-page Plates, and numerous Wood Engravings. Cloth, \$4.50; Full Leather, \$5.50

EDGAR. Text-Book of Obstetrics. By J. Clifton Edgar, M.D., Professor of Obstetrics, Medical Department of Cornell University, New York City. Elaborately Illustrated. In Press.

FULLERTON. Obstetric Nursing. 5th Ed. Illustrated. \$r.0

LANDIS. Compend of Obstetrics, 7 th Edition, Revised by WM. H. WeLLS, Demonstrator of Clinical Obstetrics, Jefferson Medical College. 52 Illustrations. .80 ; Interleaved, $\$ 1.00$

WINCKEL. Text-Book of Obstetrics, Including the Pathology and Therapeutics of the Puerperal State. Illus. $\$ 5.00$

\section{PATHOLOGY-BACTERIOLOGY.}

BARLOW. General Pathology. 795 pages. 8vo. \$5.00

BLACK. Micro-Organisms. The Formation of Poisons. $\quad .75$

BLACKBURN. Autopsies. A Manual of Autopsies Designed for the Use of Hospitals for the Insane and other Public Institutions. Ten full-page Plates and other Illustrations.

CONN. Agricultural Bacteriology. Illustrated.

$\$ 1.25$

CONN. Bacteria in Milk Products. Illustrated. In Press.

COPLIN. Manual of Pathology. Including Bacteriology, Technic of Post-Mortems, Methods of Pathologic Research, etc. 330 Illustrations, 7 Colored Plates. $3 \mathrm{~d}$ Edition. $\$ 3.50$

DA COSTA. Clinical Hematology. A Practical Guide to the Examination of the Blood. Six Colored Plates and 48 Illustrations. Just Ready.

Cloth, $\$ 5.00$; Sheep, $\$ 6.00$

EMERY. Bacteriological Diagnosis. 2 Colored Plates and 32 other Illustrations. Just Ready.

$\$ 1.50$

HEWLETT. Manual of Bacteriology. 75 Illustrations. Second Edition, Revised and Enlarged. Just Ready. $\$ 4.00$

ROBERTS. Gynecological Pathology. Illustrated. $\$ 6.00$

THAYER. Compend of General Pathology. Illustrated.

Just Ready. .80 ; Interleaved, $\$ 1.00$

THAYER. Compend of Special Pathology. Illustrated. Just Ready. .80 ; Interleaved, $\$ 1.00$

VIRCHOW. Post-Mortem Examinations, 3d Edition.

WHITACRE, Laboratory Text-Book of Pathology. with I2I Illustrations.

WILLIAMS. Bacteriology. A Manual for Students. 90 Illustrations. 2d Edition, Revised. $\$$ r.50

\section{PHARMACY.}

Special Catalogue of Books on Pharmacy sent free upon application. COBLENTZ. Manual of Pharmacy. A Complete Text-Book by the Professor in the New York College of Pharmacy. 2d Edition, Revised and Enlarged. 437 Illus. Cloth, \$3.50; Sheep, \$4.50

COBLENTZ. Volumetric Analysis. Illustrated. \$1.25 
BEASLEY. Book of 3100 Prescriptions. Collected from the Practice of the Most Eminent Physicians and Surgeons-English, French, and American. A Compendious History of the Materia Medica, Lists of the Doses of all the Officinal and Established Preparations, an Index of Diseases and their Remedies. 7 th Ed. \$2.00

BEASLEY. Druggists' General Receipt Book. Comprising a Copious Veterinary Formulary, Recipes in Patent and Proprietary Medicines, Druggists' Nostrums, etc. ; Perfumery and Cosmetics, Beverages, Dietetic Articles and Condiments, Trade Chemicals, Scientific Processes, and many Useful Tables. Ioth Ed. \$2.00

BEASLEY. Pharmaceutical Formulary. A Synopsis of the British, French, German, and United States Pharmacopœias. Comprising Standard and Approved Formula for the Preparations and Compounds Employed in Medicine. I 2 th Edition. $\$ 2.00$

PROCTOR. Practical Pharmacy. 3d Edition, with Illustrations and Elaborate Tables of Chemical Solubilities, etc. $\$ 3.00$

ROBINSON. Latin Grammar of Pharmacy and Medicine. 3d Edition. With elaborate Vocabularies. \$1.75

SAYRE. Organic Materia Medica and Pharmacognosy. An Introduction to the Study of the Vegetable Kingdom and the Vegetable and Animal Drugs. Comprising the Botanical and Physical Characteristics, Source, Constituents, and Pharmacopeial Preparations, Insects Injurious to Drugs, and Parmacal Botany. With sections on Histology and Microtechnique, by W. C. Stevens. 374 Illustrations. Second Edition. Cloth, \$4.50

SCOVILLE. The Art of Compounding. Second Edition, Revised and Enlarged.

Cloth, $\$ 2.50$

STEWART. Compend of Pharmacy. Based upon "Reming. ton's Text-Book of Pharmacy." 5th Edition, Revised in Accordance with the U. S. Pharmacopcia, 189o. Complete Tables of Metric and English Weights and Measures. .80; Interleaved, $\$ 1.00$

TAVERA. Medicinal Plants of the Philippines. $\$ 2.0$

UNITED STATES PHARMACOPGEIA. 7th Decennial Revision. Cloth, $\$ 2.50$ (postpaid, $\$ 2.77$ ); Sheep, $\$ 3.00$ (postpaid, $\$ 3.27$ ); Interleaved, $\$ 4.00$ (postpaid, $\$ 4.50$ ); Printed on one side of page only, unbound, $\$ 3.50$ (postpaid, $\$ 3.90$ ).

Select Tables from the U. S. P. Being Nine of the Most Important and Useful Tables, Printed on Separate Sheets.

.25

POTTER. Hand-Book of Materia Medica, Pharmacy, and Therapeutics. 600 Prescriptions. 8th Ed. Clo., $\$ 5 . \infty$; Sh., $\$ 6 . \infty$

\section{PHYSIOLOGY.}

BIRCH. Practical Physiology. An Elementary Class Book. 62 Illustrations.

$\$ 1.75$

BRUBAKER. Compend of Physiology. Ioth Edition, Revised and Enlarged. Illustrated.

.80; Interleaved, $\$ \mathbf{1} .00$

JONES. Outlines of Physiology. 96 Illustrations.

KIRKES. Handbook of Physiology. I th Authorized Edition. Revised, Rearranged, and Enlarged. By Prof. W. D. HalliburTON, of Kings College, London. 68I Illustrations, some of which are in colors.

Cloth, $\$ 3.00$; Leather, $\$ 3.75$ 
LANDOIS. A Text-Book of Human Physiology, Including Histology and Microscopical Anatomy, with Special Reference to the Requirements of Practical Medicine. 5th American, translated from the last German Edition, with Additions by WM. STIRLing, M.D.,D.sC. 845 Illus., many of which are printed in colors. In Press.

STARLING. Elements of Human Physiology. 100 Ills. \$1.00

STIRLING. Outlines of Practical Physiology. Including Chemical and Experimental Physiology, with Special Reference to $\begin{array}{ll}\text { Practical Medicine. 3d Edition. 289 Illustrations. } & \$ 2.00\end{array}$

TYSON. Cell Doctrine. Its History and Present State. \$1.50

\section{PRACTICE.}

BEALE. On Slight Ailments; their Nature and Treatment. 2d Edition, Enlarged and Illustrated.

FAGGE. Practice of Medicine. 4th Edition, by P. H. PyESmith, м D. 2 Volumes. Vol. I, \$6.00; Vol. II, In Press. FOWLER. Dictionary of Practical Medicine. By various writers. An Encyclopædia of Medicine. Clo., \$3.00; Half Mor. $\$ 4.00$

GOULD AND PYLE. Cyclopedia of Practical Medicine and Surgery. A Concise Reference Handbook, with particular Reference to Diagnosis and Treatment. Edited by Drs. Gould and PyLe, Assisted by 72 Special Contributors. Illustrated, one volume. Large Square Octavo, Uniform with "Gould's Illustrated Dictionary." Sheep or Half Mor., \$10 oo: with Thumb Index, $\$ 11.0$ Half Russia, Thumb Index, $\$ 1200$ 405 Complete descriptive circular free upon application.

GOULD AND PYLE'S Pocket Cyclopedia of Medicine and Surgery. Based upon the above and Uniform with "Gould's Pocket Dictionary."

Full Limp Leather, Gilt Edges, Round Corners, \$1.00

With Thumb Index, $\$ 1.25$

HUGHES. Compend of the Practice of Medicine. 6th Edition,

Revised and Enlarged.

Part I. Continued, Eruptive, and Periodical Fevers, Diseases of the Stomach, Intestines, Peritoneum, Biliary Passages, Liver, Kidneys, etc., and General Diseases, etc.

Part II. Diseases of the Respiratory System, Circulatory System, and Nervous System; Diseases of the Blood, etc.

Price of each part, .80; Interleaved, \$1.00

Physician's Edition. In one volume, including the above two parts, a Section on Skin Diseases, and an Index. 6th Revised Edition. 625 pp. Full Morocco, Gilt Edge, \$2.25

MURRAY. Rough Notes on Remedies. 4th Ed. \$x.25

TAYLOR. Practice of Medicine. 6th Edition. $\$ 4.00$

TYSON. The Practice of Medicine. By James Tyson, M.D., Professor of Medicine in the University of Pennsylvania. A Com. plete Systematıc Text-book with Special Reference to Diagnosis and Treatment. 2d Edition, Enlarged and Revised. Colored Plates and I25 other Illustrations. 1222 Pages. Cloth, $\$ 5.50$; Leather, $\$ 6.50$

\section{STOMACH. INTESTINES.}

HEMMETER. Diseases of the Stomach. Their Special Pathology, Diagnosis, and Treatment. With Sections on Anatomy, Analysis of Stomach Contents, Dietetics, Surgery of the Stomach, etc. 3d Fdition, Revised. With ${ }_{5}$ Plates and $4 \mathrm{I}$ other Illustrations, a number of which are in Colors. Just Ready

Cloth, \$6.00; Sheep, $\$ 7.00$ 
HEMMETER. Diseases of the Intestines. Their Special Pathology, Diagnosis, and Treatment. With Sections on Anatomy and Physiology, Microscopic and Chemic Examination of Intestinal Contents. Secretions, Feces and Urine, Intestinal Bacteria and Parasites, Surgery of the Intestines, Dietetics, Diseases of the Rectum, etc. With Full-page Colored Plates and many other Original Illustrations. 2 Volumes. Octavo. Just Ready.

Price of each Volume, Cloth, $\$ 5.00$; Sheep, $\$ 6.00$

\section{SKIN.}

BULKLEY. The Skin in Health and Disease. Illustrated. .40

CROCKER. Diseases of the Skin. Their Description, Pathology, Diagnosis, and Treatment, with Special Reference to the Skin Eruptions of Children. 3d Edition, Thoroughly Revised. With New Illustrations. Nearly Ready. $\$ 5.00$

SCHAMBERG. Diseases of the Skin. 2d Edition, Revised and Enlarged. 105 Illustrations. Being No. 16 ? Quiz-Compend ? Series. Cloth, .80; Interleaved, $\$ 1.00$

VAN HARLINGEN. On Skin Diseases. A Practical Manual of Diagnosis and Treatment, with special reference to Differential Diagnosis. 3d Edition, Revised and Enlarged. With Formula and 60 Illustrations, some of which are printed in colors. \$2.75

\section{SURGERY AND SURGICAL DIS- EASES (see also Urinary Organs).}

BERRY. Diseases of the Thyroid Gland and Their Surgical Treatment. Illustrated.

BUTLIN. Operative Surgery of Malignant Disease. 2d Edition. Illustrated. Octavo. $\$ 4.50$

DAVIS. Bandaging. Its Principles and Practice. 163 Original Illustrations.

$\$ 1.50$

DEAVER. Surgical Anatomy. A Treatise on Human Anatomy in its Application to Medicine and Surgery. With about $45^{\circ}$ very Handsome full-page Illustrations Engraved from Original Drawings made by special Artists from Dissections prepared for the purpose. Three Volumes. Royal Square Octavo.

Cloth, \$21.00; Half Morocco or Sheep, $\$ 24.00$; Half Russia, \$27.00 Complete descriptive circular and special terms upon application.

DEAVER. Appendicitis, Its Symptoms, Diagnosis, Pathology, Treatment, and Complications. Elaborately Illustrated with Colored Plates and other Illustrations. 3d Edition. Preparing.

DULLES. What to Do First in Accidents and Poisoning. 5th Edition. New Illustrations. $\$ 1.00$

FULLERTON. Surgical Nursing. 3d Edition. 69 Illus. \$1.00

HAMILTON. Lectures on Tumors. 3d Edition. \$1.25

HEATH. Minor Surgery and Bandaging. 12th Edition, Revised and Enlarged. I95 Illus., Formulx, Diet List, etc. \$1.50

HEATH. Clinical Lectures on Surgical Subjects. Second Series. Just Ready. $\$ 2.00$

HORWITZ. Compend of Surgery and Bandaging, including Minor Surgery, Amputations, Fractures, Dislocations, Surgical Diseases, etc., with Differential Diagnosis and Treatment. 5th Edition, very much Enlarged and Rearranged. 167 Illustrations, o8 Formulæ. Cloth, .80: Interleaved, $\$ 1.00$ 
JACOBSON. Operations of Surgery. 4th Edition, Enlarged. $55^{\circ}$ Illustrations. Two Volumes. Cloth, \$10.00; Leather, \$12.00 KEAY. Medical Treatment of Gall Stones. Just Ready. \$1.25

KEHR. Gall-Stone Disease. Translated by William Wotkyns SEYMOUR, M.D. $\$ 2.50$

MAKINS. Surgical Experiences in South Africa. 1899-1900. Illustrated. $\$ 4.00$

MAYLARD. Surgery of the Alimentary Canal. 97 Illustrations. 2d Edition, Revised. \$3.00

MOULLIN. Text-Book of Surgery. With Special Reference to Treatment. 3d American Edition. Revised and edited by JoHN B. Hamilton, M.D., Ll.D., Professor of the Principles of Surgery and Clinical Surgery, Rush Medical College, Chicago. 623 Illustrations, many of which are printed in colors. Cloth, $\$ 6.00$; Leather, $\$ 7.0$

SMITH. Abdominal Surgery. Being a Systematic Description of all the Principal Operations. 224 Illus. 6th Ed, 2 Vols. Clo., \$10.0

VOSWINKEL. Surgical Nursing. Second Edition, Revised and Enlarged. II I Illustrations.

WALSHAM. Manual of Practical Surgery. 7th Ed., Revised and Enlarged. 483 Engravings. 950 pages. $\$ 3.50$

\section{TEMPERATURE CHARTS, ETC.}

GRIFFITH. Graphic Clinical Chart for Recording Temperature, Respiration, Pulse, Day of Disease, Date, Age, Sex, Occupation, Name, etc. Printed in three colors. Sample copies free. Put up in loose packages of fifty, .50. Price to Hospitals, 500 copies, $\$ 4.00$; 1000 copies. $\$ 7.50$.

KEEN'S CLINICAL CHARTS. Seven Outline Drawings of the Body, on which may be marked the Course of Disease, Fractures, Operations, etc. Each Drawing may be had separately, twenty-five to pad, 25 cents.

SCHREINER. Diet Lists. Arranged in the form of a chart. With Pamphlets of Specimen Dietaries. Pads of 50.

\section{THROAT AND NOSE (see also Ear).}

COHEN. The Throat and Voice. Illustrated. Two Colored Plates and 80 Illustrations.

HOLLOPETER. Hay Fever. Its Successful Treatment. \$1.0 KNIGHT. Diseases of the Throat. A Manual for Students. Illustrated. Nearly Ready.

LAKE. Laryngeal Phthisis, or Consumption of the Throat. Colored Illustrations. $\$ 2.00$

MCBRIDE. Diseases of the Throat, Nose, and Ear. With colored Illustrations from original drawings. $3 \mathrm{~d}$ Edition. $\$ 7.00$

POTTER. Speech and its Defects. Considered Physiologically, Pathologically, and Remedially.

SHEILD. Nasal Obstructions. Illustrated.

\section{URINE AND URINARY ORGANS.}

ACTON. The Functions and Disorders of the Reproductive Organs in Childhood, Youth, Adult Age, and Advanced Life, Considered in their Physiological, Social, and Moral Relations. 8th Edition. 
HOLLAND. The Urine, the Gastric Contents, the Common Poisons, and the Milk. Memoranda, Chemical and Microscopical, for Laboratory Use. Illustrated and Interleaved. 6th Ed. \$1,0

KLEEN. Diabetes and Glycosuria.

$\$ 2.50$

MEMMINGER. Diagnosis by the Urine. 2d Ed. 24 Illus, \$1.00

MORRIS. Renal Surgery, with Special Reference to Stone in the Kidney and Ureter and to the Surgical Treatment of Calculous Anuria. Illustrated.

$\$ 2 . \infty 0$.

MOULLiN. Enlargement of the Prostate. Its Treatment and Radical Cure. 2d Edition. Illustrated.

$\$ 1.75$

MOULLIN. Inflammation of the Bladder and Urinary Fever. Octavo.

$\$ 1.50$

SCOTT. The Urine. Its Clinical and Microscopical Examination. 4 Lithographic Plates and other Illustrations. Quarto. Cloth, \$5.00

TYSON. Guide to Examination of the Urine. For the Use of Physicians and Students. With Colored Plate and Numerous Illustrations engraved on wood. Ioth Edition, Revised, Enlarged, and partly Rewritten. With New Illustrations. Just Ready. \$r.50

VAN NUYS. Chemical Analysis of Urine. 39 Illus. $\$ 1.0$

\section{VENEREAL DISEASES.}

GOWERS. Syphilis and the Nervous System. \$r.o STURGIS AND CABOT. Student's Manual of Venereal Diseases. 7 th Revised and Enlarged Ed. 12mo. \$1.35

\section{VETERINARY.}

BALLOU. Veterinary Anatomy and Physiology. 29 Graphic Illustrations. .80 ; Interleaved, 1.00

\section{WOMEN, DISEASES OF.}

BISHOP. Uterine Fibromyomata. Their Pathology, Diagnosis, and Treatment. Illustrated.

Cloth, \$3.50

BYFORD (H. T.). Manual of Gynecology. Third Edition, Revised and Enlarged. $36_{3}$ Illustrations. Just Ready. \$3.00

DÜHRSSEN. A Manual of Gynecological Practice. I05 Illustrations.

FULLERTON Surgical Nursing. $3 d$ Edition, Revised and Enlarged. 60 Illustrations. Nursing. 3d Edition, Revised and

LEWERS. Diseases of Women. 146 Illus. 5th Ed. $\$ 2.50$ MONTGOMERY. Practical Gyrecology. A Complete Systematic Text-Book. 527 Illustrations. Cloth, $\$ 5.00$; Leather, $\$ 6.0$

ROBERTS. Gynecological Pathology. With 127 Full-page Plates containing I5I Figures.

WELLS. Compend of Gynecology. Illustrated. 2d Edition. .80 ; Interleaved, $\$ 1.00$ 
"We know of no series of books issued by any house that so fully meets our approval as these ?Quiz-Compends?. They are well arranged, full, and concise, and are really the best line of text-books that could be found for either student or practitioner." - Southern Clinic.

\section{BLAKISTON'S ? QUIZ-COMPENDS?}

The Best Series of Manuals for the Use of Students.

Price of each, Cloth, .80. Interleaved, for taking Notes, \$1.00.

These Compends are based on the most popular text-books and the lectures of prominent professors, and are kept constantly revised, so that they may thoroughly represent the present state of the subjects upon which they treat. The authors have had large experience as Quiz-Masters and attaches of colleges, and are well acquainted with the wants of students. They are arranged in the most approved form, thorough and concise, containing nearly rooo illustrations and lithograph plates, inserted wherever they could be used to advantage. Can be used by students of any college. They contain information nowhere else collected in such a condensed, practical shape.

No. I. POTTER. HUMAN ANATOMY. Sixth Edition, II7 Illustrations and 16 Plates of Nerves and Arteries.

No. 2. HUGHES. PRACTICE OF MEDICINE. Part I. Sixth Edition, Enlarged and Improved.

No. 3. HUGHES. PRACTICE OF MEDICINE. Part II. Sixth Edition, Revised and Improved.

No. 4. BRUBAKER. PHYSIOLOGY. Tenth Edition. Illus. No. 5. LANDIS. OBSTETRICS. Seventh Edition. 52 Illus. No. 6. POTTER. MATERIA MEDICA, THERAPEUTICS, AND PRESCRIPTION WRITING. Sixth Revised Edition.

No. 7. WELLS. GYNECOLOGY. Second Ed. I40 Illus.

No. 8. GOULD AND PYLE. DISEASES OF THE EYE. Second Edition. Refraction, Treatment, Surgery, etc. rog Illus.

No. 9. HORWITZ. SURGERY. Including Minor Surgery, Bandaging, Surgical Diseases, Differential Diagnosis and Treat-4 ment. Fifth Edition. With 98 Formulæ and $7 x$ Illustrations.

No. I0. LEFFMANN. MEDICAL CHEMISTRY. Fourth Edition. Including Urinalysis, Animal Chemistry, Chemistry of Milk, Blood, Tissues, the Secretions, etc.

No. Ix. STEWART. PHARMACY. Fifth Edition. Based upon Prof. Remington's Text-Book of Pharmacy.

No. 12. BALLOU. VETERINARY ANATOMY AND PHYSIOLOGY. 29 graphic Illustrations.

No. 13. WARREN. DENTAL PATHOEOGY AND DENTAL MEDICINE. Third Edition, Illustrated.

No. 14. HATFIELD. DISEASES OF CHILDREN. 3d Ed.

No. 15. THAYER. GENERAL PATHOLOGY. 78 Illus.

No. I6. SCHAMBERG. DISEASES OF THE SKIN. Second Edition, Revised and Enlarged. ro5 Illustrations.

No. 17. CUSHING. HISTOLOGY. Illustrated.

No. 18. THAYER. SPECIAL PATHOLOGY. 34 Illustrations.

Careful attention has been given to the construction of each sentence, and while the books will be found to contain an immense amount of knowledge in small space, they will likewise be found easy reading. 


\section{DA COSTA}

\section{Clinical Hematology}

A Practical Guide to the Examination of the Blood by Clinical Methods. With Reference to the Diagnosis of Disease. With Colored Illustrations. Cloth, $\$ 5.00$

* A new, thorough, systematic, and comprehensive work, its purpose being, first, to show how to examine the blood, and second, how to diagnose from such examination diseases of the blood itself and general diseases. The author's aim has been to cover not alone the field of original research, but to supply a book for the student, the hospital physician and the general practitioner. It will be found wanting in none of these respects.

\section{OERTEL}

\section{Medical Microscopy}

NEARLY READY

A GUIDE TO DIAGNOSIS, ELEMEN-

TARY LABORATORY METHODS,

AND MICROSCOPIC TECHNIC

$$
\text { By T. E. Oertel, M.D., }
$$

Professor of Pathology and Clinical Microscopy, Medical Department, University of Georgia.

WITH 120 ILLUSTRATIONS 


\section{JACOBSON'S}

\section{Operations of Surgery}

The Operations of Surgery. By W. H. A. Jacobson, F.R.C.s., Surgeon to Guy's Hospital; Consulting Surgeon Royal Hospital for Children and Women; Member Court of Examiners Royal College of Surgeons; Joint Editor Annals of Surgery; and F. J. Steward, F.R.c.s., Assistant Surgeon Guy's Hospital and to the Hospital for Sick Children. Fourth Edition, Revised, Enlarged and Improved. 550 Illustrations. Two Volumes, Octavo, ${ }_{5} 24$ pages. Cloth, $\$ 10.00$ Sheep, \$I 2.00

\section{PRESS NOTICES OF FORMER EDITIONS}

"The author proves himself a judicious operator, as shown by his choice of methods and by the emphasis with which he refers to the different dangers and complications which may arise to mar success or jeopardize life."-Nere York Medical Record.

"The important anatomical points are clearly set forth, the conditions indicating or contraindicating operative interference are given, the details of the operations themselves are brought forward prominently, and frequently the aftertreatment is considered. Herein is one of the strong points of the book." - Neze York Medical Journal. 


\section{The Pocket Cyclopedia of}

\section{Medicine and Surgery}

Full Limp Leather, Round Corners, Gilt Edges, $\$ 1.00$ With Thumb Index, $\$ 1.25$

Uniform with "Gould's Pocket Dictionary"

A concise practical volume of nearly 600 pages, containing a vast amount of information on all medical subjects, including Diagnosis and Treatment of Disease, with Formulas and Prescriptions, Emergencies, Poisons, Drugs and Their Uses, Nursing, Surgical Procedures, Dose List in both English and Metric Systems, etc.

\section{By Drs. Gould and Pyle}

Based upon their large "Cyclopedia of

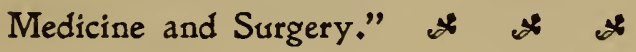

** This is a new book which will prove of the greatest value to students. It is to the broad field of general medical information what "Gould's Pocket Dictionary" is to the more special one of definition and pronunciation of words. The articles are concise but thorough, and arranged in shape for quick reference. In no other book can be found so much exact detailed knowledge so conveniently classified, so evenly distributed, so methodically grouped. It is Multum in Parvo. 


\section{A NEW EDITION}

\section{Crocker on the Skin}

The Diseases of the Skin. Their Description, Pathology, Diagnosis, and Treatment, with Special Reference to the Skin Eruptions of Children. By H. RADCliffe Crocker, M. D., Physician to the Department of Skin Diseases, University College Hospital, London. With new Illustrations.

\section{Third Edition, Rewritten and Enlarged}

\section{NEARLY READY; CLOTH, $\$ 5.00$}

* * This new edition will easily hold the bigh position given the previous printings. The author is a member of American, English, French, German, and Italian Dermatological Societies, and a recognized authority the world over.

\section{STURGIS-MANUAL OF}

\section{VENEREAL DISEASES}

By F. R. Sturgis, M.D., Sometime Clinical Professor of Venereal Diseases in the Medical Department of the University of the City of New York. Seventh Edition, Revised and in Part Rewritten by the Author and Follen CABot, M.D., Instructor in Genito-Urinary and Venereal Diseases in the Cornell University Medical College. 12mo. 216 pages.

Cloth, $\$ 1.25$

* * This manual was originally written for students' use, and is as concise and as practical as possible. It presents a careful, condensed description of the commoner forms of venereal diseases which occur in the practice of the general physician, together with the most approved remedies. 


\section{FOR THE DISSECTING ROOM}

\section{Holden's Anatomy-Seventh Edition}

\section{Illustrations}

A Manual of the Dissections of the Human Body. By Jorn LANGton, F.R.C.S. Carefully Revised by A. Hewson, M.D., Demonstrator of Anatomy: Jefferson Medical College, Philadelphia, etc. 320 Illustrations. Two small compact volumes. $12 \mathrm{mo}$.

Vol. I. Scalp, Face, Orbit, Neck, Throat, Thorax, Upper Extremity. 435 pages. I53 Illustrations.

Oil Cloth, \$1.50

Vol. II. Abdomen, Perineum, Lower Extremity, Brain, Eye, Ear, Mammary Gland, Scrotum, Testes. $4+5$ pages. 167 Illustrations.

Oil Cloth, \$I.50

Each volume sold separately.

\section{Hughes and Keith-Dissections Illustrated}

A Manual of Dissections by Alfred WV. Hughes, M.B., M.R.c.s. (Edin.), late Professor of Anatomy and Dean of Medical Faculty, King's College, London, etc., and ARTHUR KEITH, M.D., Joint Lecturer on Anatomy, London Hospital Medical College, etc. In three parts. With 527 Colored and other Illustrations.

I. Upper and Lower Extremity. $3 S$ Plates, I 6 other Illustrations. Cloth, $\$ 3.00$

II. Abdomen. Thorax. 4 Plates, I49 other Illustrations. Cloth, $\$ 3.00$

III. Head, Neck, and Central Nervous System. 16 Plates, 204 other Illustrations. Cloth, $\$ 3.00$ Each a'olume sold sefarately.

* * The student will find it of great advantage to have a "Dissector" to supplement his regular text-book on anatomy. These books meet all requirements, and as they can be purchased in parts as wanted, the outlay is small. 


\section{EDGAR'S \\ OBSTETRICS}

A NEW TEXT-BOOK

The Illustrations in Edgar's Obstetrics surpass in number, in artistic beauty, and in practical worth those in any book of similar character. They are largely from original sources, are made to a scale, and have been drawn by artists of long experience in this class of medical work.

The Text has been prepared with great care. The author's extensive experience in hospital and private practice and as a teacher, his cosmopolitan knowledge of literature and methods, and an excellent judgment based upon these particularly fit him to prepare what must be a standard work.

$$
I N \quad P R E S S
$$




\section{JUST READY}

\section{A Companion Volume to Gould's Pocket Dictionary}

\section{A POCKET CYCLOPEDIA}

OF

\section{MEDICINE $a^{n^{d}}$ SURGERY}

Eortro ar

GEORGE M. GOULD, A.M., M.D.

Author of "Gould's Medical Dictionaries ;" Editor of "American Medicine"

AND

WALTER L. PYLE, A.M., M.D.

Assistant Surgcon Wills Eye Hospital, Philadelphia; formerly Editor

"International Medical Magazine," etc.

BEING BASED UPON GOULD AND PYLE'S LARGE "CYCLOPEDIA OF PRACTICAL MEDICINE AND SURGERY"

Uniform with Gould's Pocket Dictionary. 64mo. Flexible Leather, Gilt Edges, Round Corners, net $\$ 1.00$; with Thumb Index, \$1.25

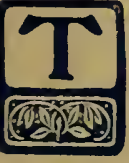

HIS book bears to Gould and Pyle's large "Cyclopedia of Medicine and Surgery" a relation similar to that which the Pocket Dictionary bears to Gould's complete "Illustrated Dictionary." As the Dictionary gives the derivation, pronunciation, and definition of medical words, the Cyclopedia is designed to furnish general information concerning medical subjects. Every subject, concerning which the student may desire a brief and thorough description, supplementing the mention which may be given in lectures or a general text-book, is taken up and treated thoroughly and concisely. To those desiring concise authoritative information on medical or surgical themes or who wish to look up any new term or matter of recent discovery and use, the book will prove invaluable. It includes articles on Emergencies, Hygiene, Poisons, Nursing, etc.; describes Drugs and their Uses; gives Treatment of Diseases; explains Surgical Operations; contains many Prescriptions and Formulæ, Tables of Differential Diagnosis, Dose Table in both English and Metric Systems, etc.

\section{P. BLAKISTON'S SON \& CO., Publishers and Booksellers 1012 WALNUT STREET, PHILADELPHIA}




\section{FOR MEDICAL STUDENTS.}

\section{COLUMBIA UNIVERSITY}

This book is due on the date indicated below, or at the expiration of a definite period after the date of borrowing, or as provided by the rules of the Library or by special ar- 5 rangement with the Librarian in charge.

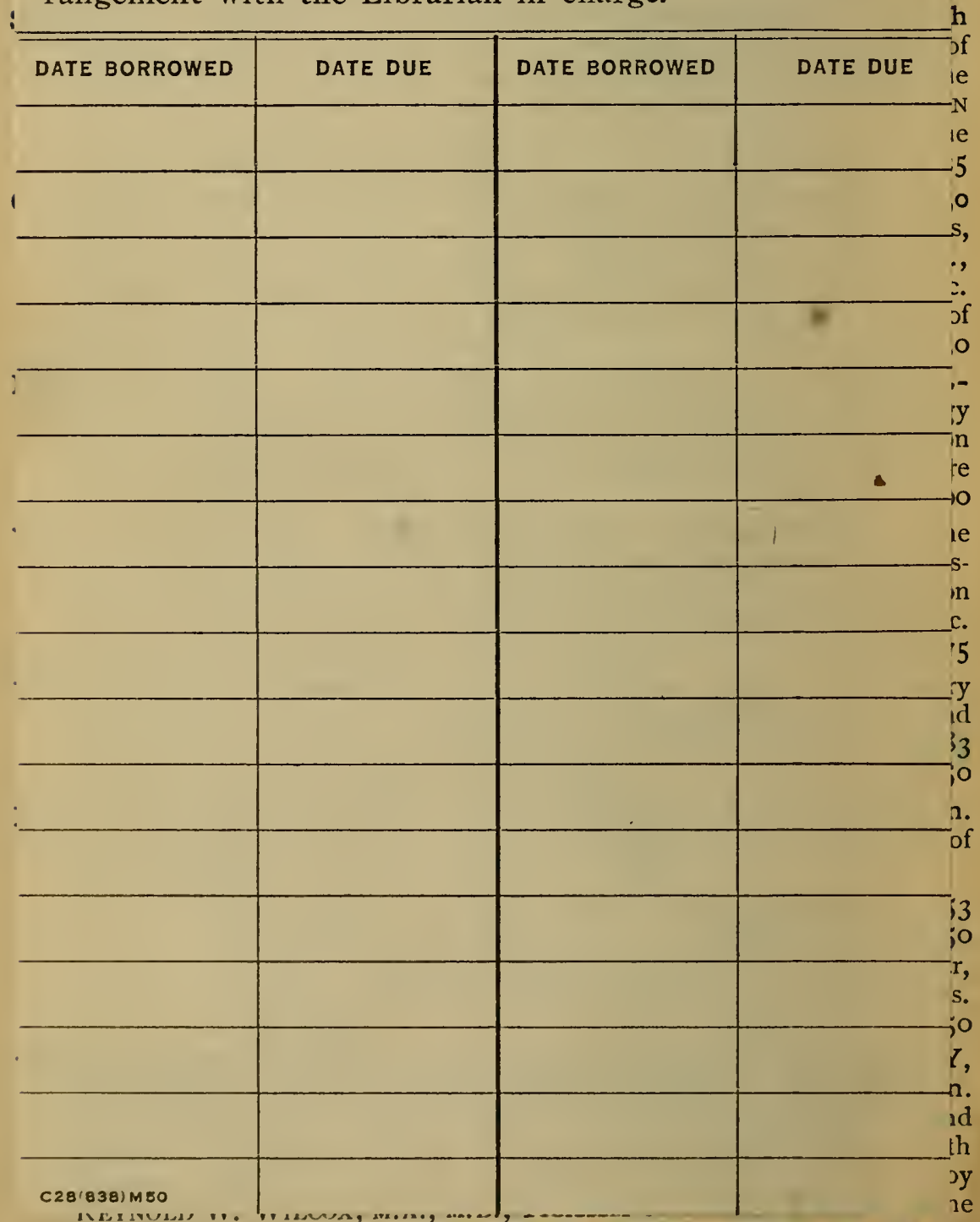

New York Post-Graduate Medical Sichool and Hospital. Cloth, net, \$3.00

P. BLAKISTON'S SON \& CO., Publishers and Booksellers, 1012 Walnut Street, Philadelphia. 


\section{FOR MEDICAL STUDENTS.}

KIRKES' PHYSIOLOGY. Seventeenth Edition. (The Authorized Edition. I2mo. Dark Red Cloth.) Revised and Enlarged. By W. D. Halliburton, M.D., F.R.S., I'rofessor of Physiology. King's Collecre, Londqn Ge. r11.... .

STÖHR. OO MICF of Ana wit?

QP40

ve

B83

1902 '

S

HA

Brubaker

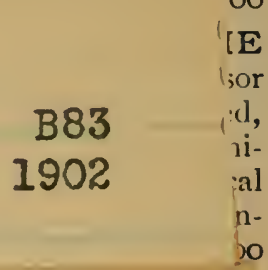

WI

REI

sw

BYH

BAF

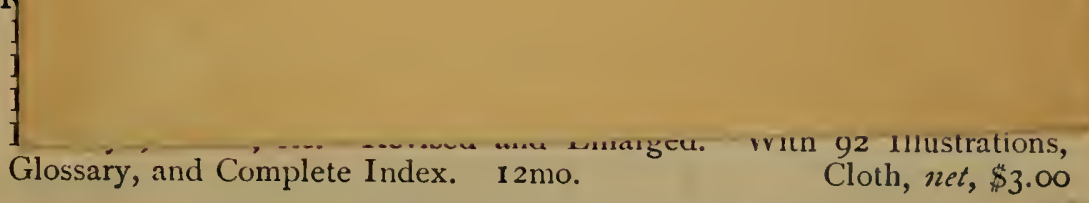

P. BLAKISTON'S SON \& CO., Publishers and Booksellers, 1012 WalNut StREet, PHILAdELPhIA. 
$x^{2}-8 \times 28$

$-\frac{10}{2}$

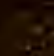

$-$

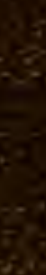

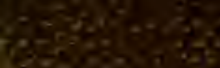

